\title{
Performance Analysis of Next-Generation LADAR for Manufacturing, Construction, and Mobility
}

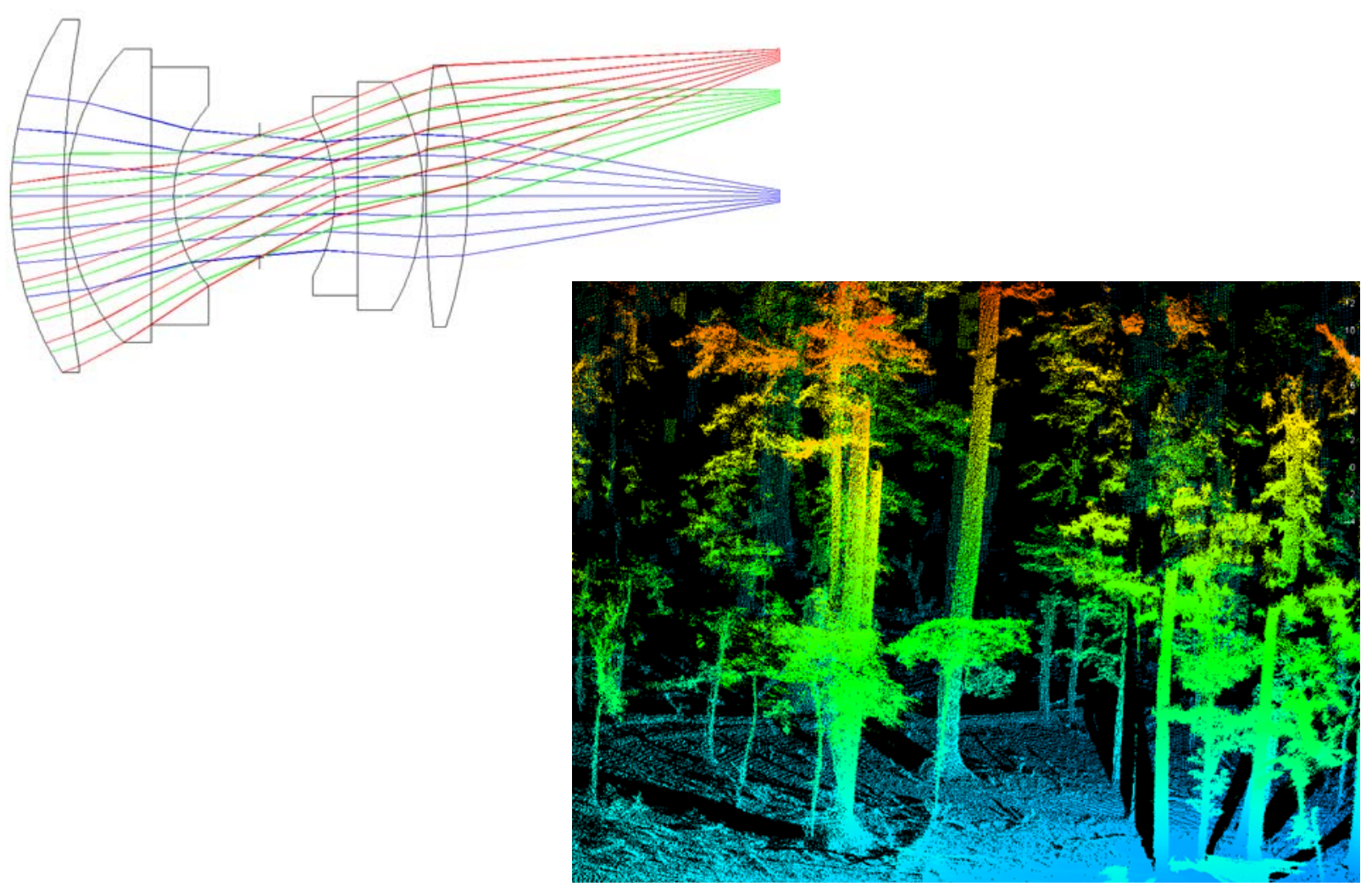

Building and Fire Research Laboratory Manufacturing Engineering Laboratory

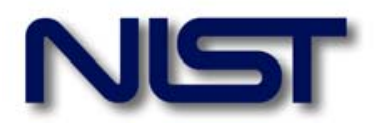

United States Department of Commerce Technology Administration

National Institute of Standards and Technology 
Cover Images: (left): Zeemax* optics program output for a candidate aspheric lens for a prototype FPA detector; (right) wooded terrain at NIST acquired at $20 \mathrm{~mm}$ accuracy and 0.24 degree angular spacing using a Rieg|* $Z-210904 \mathrm{~nm}$ wavelength scanner. The image is mapped from the native polar coordinate system of the sensor $(r, \theta, \phi=$ range, pan angle, tilt angle) to a false-color bitmap where the computed elevation has been replaced with a linearly-applied RGB variation for height cueing (blue = lowest; red $=$ highest) and the $\theta, \phi$ discrete angle steps map to column number and row number, respectively. The image was displayed using software developed at NIST running on Open/DX

* Certain trade names and company products are mentioned in the text or identified in an illustration in order to adequately specify the software, experimental procedure, or equipment used. In no case does such an identification imply recommendation or endorsement by the National Institute of Standards and Technology, nor does it imply that the products are necessarily the best available for the purpose. 


\section{Performance Analysis of Next-Generation LADAR for Manufacturing, Construction, and Mobility}

NISTIR 7117

William C. Stone

Building and Fire Research Laboratory

Maris Juberts, Nick Dagalakis, Jack Stone, Jason Gorman

Manufacturing Engineering Laboratory

May 2004

Building and Fire Research Laboratory

National Institute of Standards and Technology

Gaithersburg, Maryland 20899

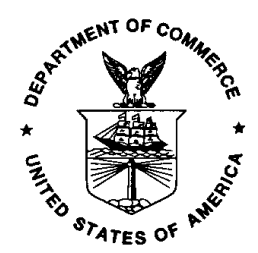

U.S. Department of Commerce

Donald L. Evans, Secretary

Technology Administration

Phillip J. Bond, Under Secretary

National Institute of Standards and Technology

Arden L. Bement, Director 


\begin{abstract}
The NIST Construction Metrology and Automation Group (CMAG), in cooperation with the NIST Intelligent Systems Division (ISD), is developing performance metrics and researching issues related to the design and development of a Next-Generation LADAR (laser radar) sensor that will enable general automation in structured and unstructured environments. This report reviews the basic physics and implementation of various LADAR technologies, describes the problems associated with available "offthe-shelf" LADAR systems, summarizes world-wide state-of-the-art research, and elaborates on general trends in advanced LADAR sensor research and their likely impact on manufacturing, autonomous vehicle mobility and on construction automation.
\end{abstract}

KEYWORDS: AM-CW; angular resolution; beam steering; digital mirror devices; field-of-view (FOV); FM-CW; focal plane array (FPA); laser radar (LADAR); phase-based time-of-flight; pulse time-of-flight; range accuracy; range resolution; scanning. 


\section{Table of Contents}

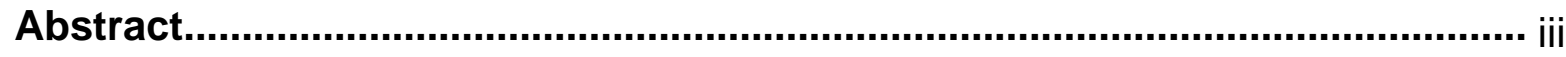

Table of Contents $\quad$ iv

1.0 Introduction.............................................................................................. 1.1

$\begin{array}{ll}1.1 \text { Background } & 1.1\end{array}$

1.2 Definition of Terms 1.3

$\begin{array}{ll}1.3 \text { Project Objectives } & 1.6\end{array}$

2.0 A Brief LADAR Physics and Technology Overview.................................. 2.1

2.1 Direct Time-of-Flight (TOF) 2.2

2.1.1 Limitations of Direct TOF 2.4

2.2 Phase-Based AM-CW 2.9

2.2.1 Range Determination in AM-Homodyne LADAR 2.13

2.2.2 Limitations of AM Phase Measurements 2.18

$\begin{array}{ll}2.3 \text { Chirped AM-CW } & 2.20\end{array}$

2.4 Coherent LADAR $\quad 2.27$

2.4.1 Hardware Variations $\quad 2.29$

2.4.2 Range Accuracy for Coherent Laser Radar 2.32

3.0 Beam Scanning Technology...................................................................... 3.1

3.1 Single Beam Scanners $\quad 3.1$

3.1.1 Polygonal Scanners $\quad 3.1$

3.1.2 Galvanometric Scanners $\quad 3.2$

3.1.3 Acousto-Optic Scanners $\quad 3.3$

3.1.4 Electro-Optic Scanners $\quad 3.3$

3.1.5 Holographic Scanners $\quad 3.3$

3.1.6 Tilt Platform Scanners $\quad 3.3$

3.2 Beam Array Scanners $\quad 3.4$

3.2.1 Scanning Micro Mirror Beam Arrays $\quad 3.4$

3.2.2 Binary Micro Mirror Beam Arrays $\quad 3.9$

3.2.3 Micro Positioner Beam Arrays 3.9

3.2.4 Micro Crystal Beam Arrays $\quad 3.12$

3.2.5 Thermo Optic Beam Array Switches $\quad 3.12$

$\begin{array}{ll}3.2 .6 \text { Liquid Crystal Beam Switches } & 3.12\end{array}$

3.3 Fast Beam Steering $\quad 3.13$

$\begin{array}{ll}3.4 \text { Risley Prisms } & 3.20\end{array}$ 
4.0 Measurement Limitations Imposed by the Refractive Index of Air.............. 4.1

4.1 Refractive Index: General considerations 4.1

4.2 Ranging Errors: Magnitude of the Problem 4.2

4.3 Correcting range measurements for refractive index 4.3

in regions of varying air temperature

4.4 Angular Errors: Magnitude of the Problem 4.7

4.5 Correcting angular measurements for refractive index variation $\quad 4.10$

4.6 Atmospheric Turbulence $\quad 4.12$

4.7 Summary of refractive index effects $\quad 4.14$

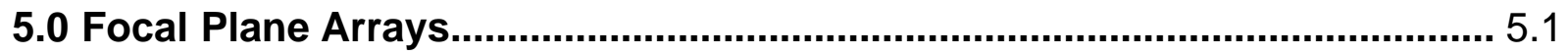

5.1 All CMOS Solutions $\quad 5.2$

5.1.1 CMOS Readout Techniques $\quad 5.5$

5.1.1.1 Floating Diffusion Readout $\quad 5.6$

5.1.1.2 Current Output Sensing Readout $\quad 5.8$

5.2 Image Tube Solutions $\quad 5.11$

$\begin{array}{ll}5.3 \text { APD Solutions } & 5.17\end{array}$

5.3.1Timing $\quad 5.20$

5.3.2 Fabrication $\quad 5.27$

5.3.3 Compensating for Low Detector Fill Factor $\quad 5.29$

5.4 Z-Stacking and VISA Solutions $\quad 5.31$

5.5 VISA Chip Research $\quad 5.40$

5.6 Coherent Solutions $\quad 5.42$

\subsection{Status Report on NIST BAA:}

Next Generation LADAR for Driving Unmanned Ground Vehicles............... 6.1

6.1 Background 6.1

6.2 BAA Status Report $\quad 6.3$

6.3 Synopses of Designs 6.4

6.3.1 Advanced Scientific Concepts Inc. (ASC) 6.4

6.3.2 Coherent Technologies Inc. (CTI) 6.5

6.3.2.1 Synopsis of the Architectural Approach 6.5

6.3.2.2 Advantages of the Approach 6.6

6.3.2.3 Technical Feasibility 6.8

6.3.2.4 Cost-Scaling, Power-Scaling, Upgrades 6.8

6.3.3 Lockheed Martin Missiles \& Fire Control $\quad 6.9$

6.3.4 Raytheon Missile Systems $\quad 6.11$

$\begin{array}{ll}6.4 \text { Strategy for Further Development } & 6.14\end{array}$

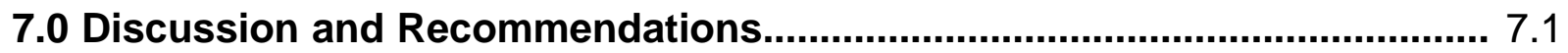

\begin{tabular}{ll}
7.1 FOV Limitations & 7.1 \\
\hline
\end{tabular}

$\begin{array}{ll}7.2 \text { Timing Issues } & 7.2\end{array}$

7.3 Illumination Source Limits $\quad 7.4$

7.4 Performance Metrics and 3D Scene Generation Issues 7.5

$\begin{array}{ll}7.5 \text { Recommendations for further Research } & 7.10\end{array}$ 


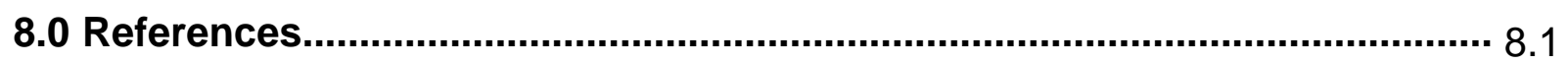

8.1 General References

8.1

8.2 Micromirror References

8.2

8.3 Coherent LADAR References

8.2

8.4 Scanning References

8.3

8.5 ROIC and Focal Plane Array (FPA) References

8.5

8.6 Super Resolution References

8.5

8.7 Femtosecond Laser References

8.6

8.8 Atmospheric Effects

8.6

Appendix A: NIST Survey of LADAR Technology 2003................................ A.1 Appendix B: NIST BAA Next-Generation LADAR....................................... B.1 


\section{Acknowledgement}

The authors would like to thank a number of individuals and organizations for freely sharing information regarding their LADAR design and development research. In particular we wish to thank Stefan Baten of EADS Dornier for insights into their mobility LADAR; to Peter Seitz and Nicolas Blanc at CSEM for their tour-de-force lecture on photonic mixing concepts; to Bernd Buxbaum and Robert Lange of PMD Technologies $\mathrm{GmbH}$ for further discussions on 4-tap AM-CW detection; to Barry Stann, Bill Ruff, and Greg Sztankay at the Army Research Lab (ARL) for explaining their work in Chirped AM-CW LADAR; to Ward Trussel and Nick Barr at the Army Night Vision Lab for sharing their insights on multiple-return system design; to Colin Smithpeter and Bob Nellums of Sandia Labs for their discussions on real-time AM-CW LADAR as well as their work on pure pulse time-of-flight Flash LADAR; to Bob Dillon at Lumen Labs, Inc. for discussions on fast light shutters; to Dave Ludwig of Irvine Sensors for explaining chip stacking; to Richard Marino, Brian Aull, Dan Fouche, and Rick Heinrichs of Lincoln Lab for explaining the inner workings of their Flash LADAR designs; to Tony Slotwinski at Metric Vision and Phil Gatt and Duane Smith at Coherent Technologies Inc. for discussions on the mathematics of coherent LADAR range accuracy; and to Roger Stettner of Advanced Scientific Concepts for material on image tube FPA systems. Many of the above individuals generously gave permission to utilize images and figures relating to their work in this report. Where used these are credited to the source institution. 


\subsection{Introduction}

\subsection{Background}

This document summarizes a general technology assessment for laser radar conducted at NIST in response to the common needs of the Building and Fire Research Laboratory's (BFRL) Construction Integration and Automation (CONSIAT) program and those of the Manufacturing Engineering Laboratory's programs for automation in manufacturing and autonomous vehicle mobility. Both groups had been making daily use of laser radar (LADAR) sensors prior to this study for significantly different reasons and both found the limitations of existing LADAR technology to be a controlling factor to furthering their independent research programs.

In the former case, LADAR was being used for construction site characterization for the purpose of controlling construction machinery and also to generate web-based billable quantities for earthmoving operations [Cheok and Witzgall, 2002]. One sigma, $\sigma$, uncertainties on the order of $+/-25 \mathrm{~mm}$ with a range of $350 \mathrm{~m}$ were obtainable but dense scans required acquisition times of $20 \mathrm{~min}$ to $90 \mathrm{~min}$ per range image. The research team working on automated steel construction at NIST [Lytle et.al., 2002] needed a real-time 3D sensor that could be used for docking steel beams at millimeter-level accuracy. "Real-time," in the context of this report means frame update rates of $10 \mathrm{~Hz}$ or greater. Likewise, size, weight, and cost are key issues for ubiquitous use on a construction site. Present commercial LADARs run anywhere from $\$ 25,000$ to in excess of $\$ 400,000$ with $\$ 100,000$ being the average cost. Sizes vary but almost none have volumes less than $15,000 \mathrm{cc}$ with masses in excess of $15 \mathrm{~kg}$. Although these can be hand carried and set on a tripod, they were not at all in the range of the ubiquitous "coffee cup-sized sensor" that the roboticists had in mind.

The Manufacturing Engineering Laboratory (MEL), on the other hand, had LADAR units mounted on autonomous vehicles that were being driven in rugged, off-road conditions, including forests, to test mobility metrics. In the application, LADAR systems proved essential for obstacle avoidance. MEL used a different class of military LADAR that achieved $30 \mathrm{~Hz}$ frame rates but did so at the cost of accuracy (about +/$150 \mathrm{~mm}$ ) and a reduced Field of View (FOV) of $+/$ - 40 degrees by $+/-10$ degrees. The narrow FOV forced the team to design an inertially stabilized "nodding" platform in order to "see" areas closer to the vehicle, such as potholes in a road in between longer look scans to see what was coming down the path. The size of these units were on the order of $35,000 \mathrm{cc}$ with a commensurate mass of $35 \mathrm{~kg}$. This robotics team reached the similar conclusion that smaller, more accurate sensors with a wider FOV were needed without losing the required frame rate.

As a result of a four year effort in support of the Army Research Lab Demo III Unmanned Ground Vehicle (UGV) program, the research team working on the mobility program at NIST has identified the needs of high performance LADAR sensors for autonomous on and off-road driving. In June of 2002, NIST released a Broad Agency 
Announcement (BAA) for the design of next generation LADARs for driving unmanned ground vehicles. The LADAR sensors were to be mounted on unmanned military robotic vehicles in order to provide range image information about the environment through which the vehicle is driving through with sufficient resolution and image quality to provide reliable and robust obstacle/object detection and terrain feature information.

Phase I of the solicitation was for concept designs which could meet the desired performance and size requirement at a reduced cost in comparison with current limitations. Appendix B contains the original published BAA announcement on the requirement specifications and Section 6.0 is a status report on the results of Phase I.

A joint task force was subsequently established at NIST to investigate the possibility of developing a compact, high resolution, fast frame rate Next-Generation LADAR (NGL) and developing the procedures and instrumentation to characterize the performance metrics of such a device. This report represents the findings of that team. 


\subsection{Definition of Terms}

The following terms are used throughout this report. They are the acronyms and jargon of the most commonly used nomenclature of the discipline. Terms in bold text within the context of a definition are those for which explicit definitions are presented elsewhere in this section. We will provide verbal descriptions here; more complete technical discussions of many of these terms appear in later chapters where appropriate in describing a particular approach to the development of LADAR systems.

APD: Avalanche Photo Diode. A variation of a $p-n$ junction photodiode that is designed to accomodate high electric fields. Light impinging on an APD creates electron-hole pairs. The electron is subsequently accelerated by the electric field. When it impacts the crystal lattice of the semiconductor substrate it generates further electronhole pairs, in effect amplifying the signal. This technique is commonly used in low light environments to detect the arrival of photons. The main development efforts in APDs have been as fiber optic terminators in telecommunications. The bandwidth of an APD detector is the number of unambiguous distinct signal measurements it can make per second. APDs can be fabricated using a number of chemistries, including traditional CMOS, but current efforts are moving towards InGaAs (Indium Galium Arsenide) and InP (Indium Phosphide) due to their preferred operational optimum wavelength which is proximal to the eyesafe $1.5 \mu \mathrm{m}$ zone, thus allowing more power to be used at the photon source.

CCD: Charge Coupled Device. imaging principle where individual pixels in an array are allowed to transport, store, and accumulate optically-generated charge carriers to defined sites within the device. CCD principles, combined with on-chip timers for each pixel can be used to create a time-of-flight focal plane array.

Flash: A generic term for a LADAR system comprised of a broad field illumination source (commonly a laser, but for close proximity it can be a bank of LEDs) and an FPA detector, such that the range image is completely acquired simultaneously in one burst. Although in some applications scanners can achieve real-time frame rates, only flash LADARs can achieve very high frame rates. In general, because of the limited number of pixels available on an FPA (currently about 256x256 is the maximum in the laboratory) flash LADARs are unable to achieve the pixel density of a scanner. However, hybrids are being developed in which flash LADARs themselves become the instrument that is mounted e.g. in a pan-tilt platform or beam steering mechanism.

FOV: Field of View. The angular mapping of the LADAR frame. Some scanned LADARS can achieve nearly full $2 \pi$ steradian coverage; most FPA systems achieve much smaller fields of view (e.g. $40^{\circ} \times 40^{\circ}$ ) as do real-time scanning LADARs. The FPA limit is determined by lens optics; the real-time scanner limit is determined by the required update rate and the speed of discrete LADAR range measurements. 
FPA: Focal Plane Array. A 2-D "chip" in which individually addressable photo sensitive "pixels" can be accessed. Early FPA detectors were developed as, first, infrared imagers and later as FLIR (Forward Looking InfraRed) detectors, largely for military purposes. CCD FPAs in the early 1960s made it possible to consider "on chip" processing electronics, making it possible to multiplex the signals from a large imaging array in real-time. These were not, however, ranging devices. Additional electronics must be added to an FPA in the form of timing circuitry. This costs "real-estate" and tends to limit the size of the array. While current CCD video camera chips can have an FPA with in excess of $1000 \times 1000$ pixels, the best operational LADAR FPA is presently a $32 \times 32$ array, although several labs have ongoing work to develop $128 \times 128$ and $256 \times 256$ arrays.

Frame: A complete 3-D data sample of the world produced by a LADAR taken at a certain time, place, and orientation. A distinguishing aspect of a LADAR over a laser rangefinder is that the LADAR produces a 3-D image of the world whereas the rangefinder supplies a single distance between two points. Raw LADAR data is almost always obtained in polar coordinates (i.e., angle, angle, range). The "image" can be represented as a mapping of the vertical and horizontal angles to $\mathrm{y}$ and $\mathrm{x}$ pixel locations, respectively, on a 2-D pixel map with the color of each pixel representing the range. The size of the frame is determined by the FOV in the horizontal and vertical directions divided by their respective angular resolutions. A single LADAR frame is also referred to as a range image.

Frame Rate: The number of LADAR frames that can be acquired per second. For example, if 10 frames could be acquired in one second, the frame rate would be 10 $\mathrm{Hz}$. This is generally a metric that is only applicable to real-time systems, since most commercial LADAR systems have update rates on the order of $20 \mathrm{~min}$ to $90 \mathrm{~min}$.

LADAR: Laser Detection and Ranging, or Laser Radar. A device consisting of a photon source (frequently, but not necessarily a laser), a photon detection system, a timing circuit, and optics for both the source and receiver. Distance from the device to targets struck by the emitted photons is measured by the time-of-flight (TOF) divided by the speed of light. Strictly speaking the device could be a single shot "0-D" measurement system (range only), but these are more commonly referred to as laser rangefinders. LADAR, on the other hand, is generally assumed to generate a 3-D Range Image.

Mixed pixels: Originally developed to describe an FPA in which portions of the unit cell were fabricated using CMOS technology while others were fabricated using, e.g. III-V InGaAs technology.

NGL: Next-Generation LADAR. Small ("coffee cup sized"), economical, high accuracy devices that can be used at high frame rates for machine control and automated field metrology applications at less than $100 \mathrm{~m}$ range. 
Range Measurement Accuracy: The uncertainty in a range measurement, usually expressed in $\mathrm{mm}$. This is a metric associated with each physical LADAR instrument. It can only be established at a calibration facility by taking scores, hundreds, or thousands of measurements across a precisely known distance and to a target with a specified degree of reflectivity. A more rigorous, mathematical definition is given in Chapter 2.

Range Measurement Precision: The deviation of a group of range measurements about a mean value (not necessarily the true value). In essence it is a measure of the repeatability of the instrument. A more rigorous, mathematical definition is given in Chapter 2.

Range Resolution: The smallest distance separation between two distinct objects illuminated by a LADAR source that can be detected in the signal return. In large part this term is controlled by the bandwidth of the receiver.

ROIC: Read-Out Integrated Circuit. In infrared imaging sensors this was a physically separate chip that resided beneath the detector FPA and provided the signal processing needed to obtain a usable image. It included such things as transimpedence amplifiers (to boost the signal strength), analog to digital converters, and frame capture electronics. In LADAR applications an additional capability must be added: timing circuitry. Many architectures have been developed in the past 30 years. Almost all of the current LADAR FPA signal processing approaches stem from concepts originally develped for FLIR detectors. The timing circuitry, on the other hand, has developed along several complicated paths that are LADAR-specific. A sampling of these architectures are discussed in detail later in this report.

Scanner: Any physical mechanism that provides the capability for a laser rangefinder to create a LADAR frame. Many different scanning mechanisms exist. The simplest to understand is a "pan-tilt" platform to which a fast laser rangefinder has been affixed. The entire instrument is tilted in discrete steps at the angular resolution of the mechanism while range measurements are made to acquire a vertical line of points. Then the entire instrument is panned by an amount equal to the angular resolution of the panning mechanism and a new vertical line is acquired. This process repeats until the entire LADAR frame is obtained. A faster approach is to use mirrored beam deflectors that can sweep the beam both horizontally and vertically. Mirrored systems make use of discrete angle planar mirrors or spinning mirrored polygons. Other optical approaches involving lenses and rotating prisms have been used as well to create the same effect.

Unit Cell: The view of an FPA as a stack (or column) of discrete functions underlying each pixel that include such basic requirements as photon detection, signal amplification, signal processing, analog to digital conversion, frame capture, and timing. 


\subsection{Project Objectives}

NIST has for a number of years conducted research in autonomous robotic platforms and machinery and has reached a consensus regarding the required performance of a "vision" system needed for effective control of machinery, be it an unmanned mobility platform or a construction crane. A LADAR meeting these criteria would have the following attributes:

Table 1: Ideal Attributes of Ground Mobility and Machine Automation LADAR-based Vision Systems

\begin{tabular}{|l|l|}
\hline Attribute & Value \\
\hline Illumination Source & Eye Safe $(1500 \mathrm{~nm})$ \\
\hline Field of View & $90^{\circ} \times 90^{\circ} ; 40^{\circ} \times 90^{\circ} ; 9^{\circ} \times 9^{\circ}$ \\
\hline Range Resolution & $1 \mathrm{~mm} \mathrm{@} 15 \mathrm{~m} ; 3 \mathrm{~mm} @ 5 \mathrm{~m}$ to $100 \mathrm{~m}$ \\
\hline Angular Resolution & $<0.03^{\circ}$ \\
\hline Frame Rate & $>10 \mathrm{~Hz}$ \\
\hline Size & "Coffee Cup" \\
\hline Cost & $<\$ 1000$ U.S. \\
\hline
\end{tabular}

Some of these individual criteria can be met by certain existing systems. Taken together, however, there is no present solution that resolves the core (second through fifth) criteria at any size or price. The first FOV requirement is for construction machinery; the second and third are for peripheral and foveal mobility needs, respectively.

Frequently there is a tradeoff between speed and accuracy. LADAR frames can be created by scanning high resolution laser rangers (see Scanner) in which a singledegree-of-freedom laser rangefinder is mechanically swept over the scene using either encoder-equipped pan/tilt servos or a rotating mirror combined with either a pan or tilt servo. There are many scanner variants ${ }^{1}$ though due to mechanical reliance these systems have inherent speed and accuracy limitations associated with the servos, the physical mechanisms, and their encoders. There are novel approaches being investigated at NIST for high-speed, compact beam deflection that may allow fast 0-D LADARs to be used for real-time, narrow FOV applications. As well, new work in FPA design shows promise for both improving range resolution as well as speed. And only this latter approach shows promise for the level of miniaturization needed to achieve the last two criteria listed above.

1 For an excellent summary up through 1993, see "Optomechanical Scanning Applications, Techniques, and Devices," by Jean Montagu and Herman DeWeerd, Chapter 3, Vol. 3, of the SPIE Infrared \& Electro-Optical Systems Handbook. 
The FOV and angular resolutions listed in Table 1 translate to a 3000×3000 pixel FPA for the largest FOV listed. The largest range-imaging FPA yet to be demonstrated successfully is $124 \times 160$ pixels [the CSEM SR-2, see Appendix A], although several labs have $128 \times 128$ pixel arrays under development and one claims to have a 256x256 pixel array in development. There are other significant optical issues relating to FPAs. Illuminating a large FPA FOV requires considerable laser power, possibly exceeding eyesafe output levels at most of the compatible wavelengths. Scanned, smaller FPAs provide possible solutions that will be discussed in detail later.

There are several additional factors currently limiting the development of high frame rate LADARs for precision metrology and machine control. All LADARs base their range determination on some form of time-of-flight (TOF) measurement, either by edge triggered synchronized differential time measurement, or by phase correlation between the outbound and incoming signals. The major sources of error are from the precision with which the initial laser pulse is generated (continuous wave, pulsed, or chirped); the nature and ambiguity of the light detection system (the current state-of-the-art involves CMOS and III-V semiconductor (e.g. InGaAs) avalanche photodiode arrays; and the means for precise timing of these events (integrated into the ROIC in the case of an FPA). Ultimately, quantum efficiency and thermal noise (dark current) have been the limiting factors in present detection systems. We attempt to discuss each of these issues in this report and suggest, where possible, potential avenues to improve the speed, accuracy, size and weight reduction of LADAR systems.. 


\subsection{A Brief LADAR Physics and Technology Overview}

Laser Detection and Ranging (LADAR) is currently poised to become the ubiquitous 3D spatial measurement tool in many disciplines. Initially used for remote sensing and aerial surveying, LADAR applications now include reverse engineering (3D models), ground surveys, automated process control, target recognition, and autonomous machinery guidance and collision avoidance to name just a few. Efforts are currently underway at NIST to develop national artifact-traceable LADAR calibration facilities; to develop rapid, LADAR-based long range autoID systems; and to establish the scientific and engineering underpinning needed to develop miniature, high resolution next-generation LADAR systems.

The power of LADAR lies in the inherent 3-D nature of the data it produces, namely spatial coordinates associated with each pixel in a so-called "range image" acquired by the device. A range image is effectively a spherically acquired $(r, \theta, \varphi)$ dataset mapped to a 2-D matrix, or "frame." LADAR frames are frequently presented as false color depth images and in 3-D using stereo displays. Additional data, including reflectance intensity associated with each pixel and multi-spectral intensity information, are commonly available. Color reflectance intensity (as opposed to active illumination frequency-specific reflected intensity) is obtained from co-boresighted RGB CCD sensors. Such a wealth of information can be rapidly segmented for use by a wide variety of real-time systems for machine control and post-processed for such metrology applications as as-built geometry checking for buildings and other civil infrastructure. This said, why are we not seeing LADAR systems on every construction site? The reasons most frequently cited are: slow speed of operation, bulky, high cost, and widely varying accuracy that presently lacks standardized calibration metrics. There are other related issues such as methods for processing the data (both real-time and offline) but that subject will not be addressed in this report.

Frequently the performance of a LADAR is defined by the following metrics:

- Maximum permissible illumination power

- Sensor horizontal Field of View (FOV)

- Sensor vertical FOV

- Wavelength of optical source

- Maximum distance to be measured

- Measurement time / frame rate

- Measurement resolution (depth)

- Measurement resolution (angular)

- Range Measurement accuracy 
To these one frequently must consider:

- Intensity of background (passive) illumination

- Color temperature of the (passive) background

- Target reflectivity (texture, color, specularity)

- Angle of beam incidence on the object

- Overall size (volume) of the sensor

- Manufactured cost of the sensor

At the conclusion of this paper we will present a set of design criteria we feel are representative of those needed to achieve ubiquitous use of LADAR sensing for construction operations. We will also comment on the research needed to achieve a physical sensor meeting such criteria.

\subsection{Direct Time-of-Flight (TOF)}

Figure 2.1.1 shows a "family tree" of LADAR devices that have at one time or another been built to operate at optical and near-optical wavelengths. Useful in-depth discussions on many of these devices are provided in [Lange, 2000; Besl, 1988; and Kammerman, 1993]. No attempt will be made here to duplicate these previous efforts, but rather, to summarize some of the basic principles and to extrapolate and expand them based on more recent hardware and software developments.

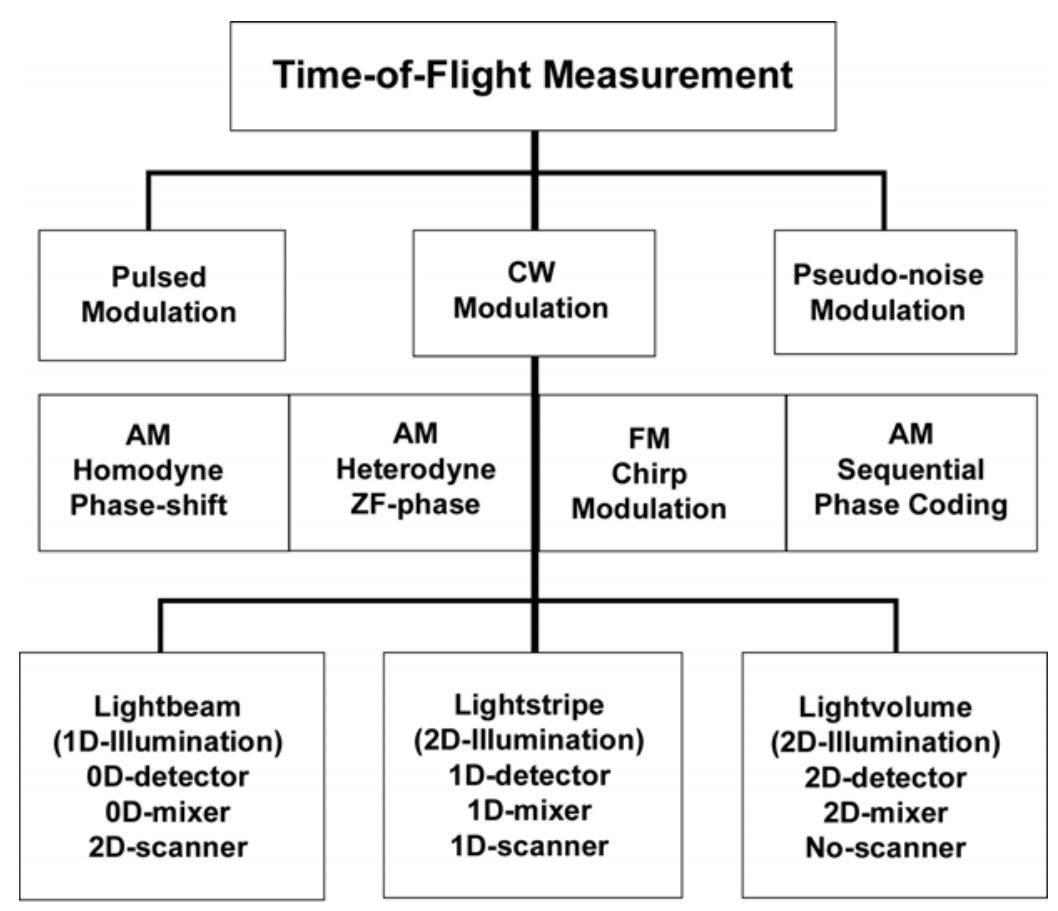

Figure 2.1.1: "Family Tree" of optical and near-optical wavelength time-of-flight range measurement devices (adapted from Lange, 2000). 
The simplest of the concepts uses direct time-of-flight (TOF) incoherent design, as illustrated in Figure 2.1.2. An illumination pulse is generated, frequently by means of a Nd-YAG microchip laser -- but any short pulse laser will work, and the time of this event is made available to a timing circuit. The beam traverses a distance equal to $2 d$ and arrives at a photonic detector in time:

$$
t=\frac{2 d}{c}
$$

where $\mathrm{c}=$ speed of light. A clock time of $1 \mathrm{~ns}$ represents a $300 \mathrm{~mm}$ round-trip flight or an absolute range of $150 \mathrm{~mm}$. One can immediately see that an extremely accurate clock is required to achieve a level of accuracy sufficient for autonomous fabrication.

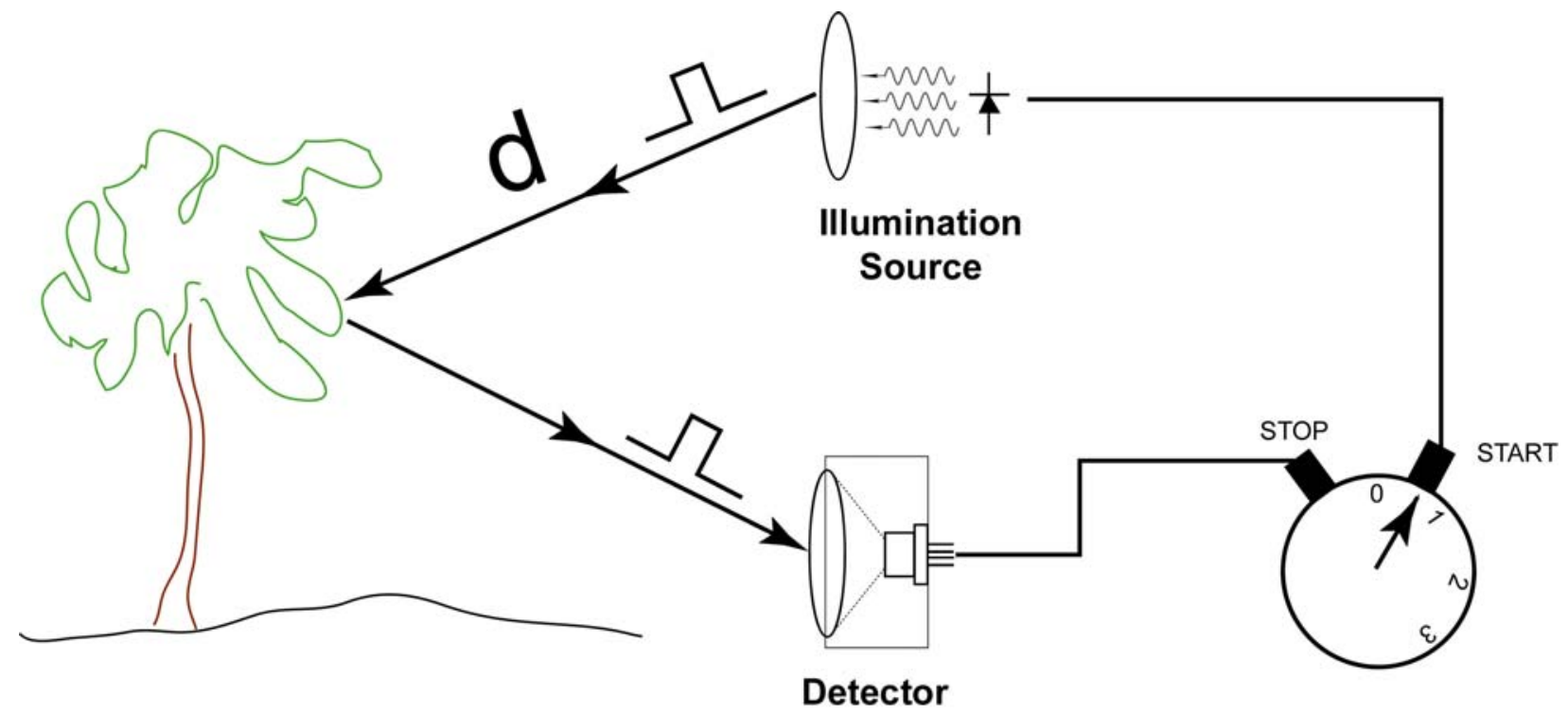

Figure 2.1.2: Fundamental physics of pure "pulsed" time-of-flight. Key performance metrics are synchronization precision of pulse initiation between the source and detector, pulse width and power, and detector bandwidth. (adapted from Lange, 2000)

Figure 2.1.3 shows a typical physical implementation of a direct TOF LADAR [Schilling and Barr et al, 2002]. The microchip laser generates 1 ns pulses at a rate of $10 \mathrm{kHz}$, producing an unambiguous range interval of $15 \mathrm{~km}$. The signals are detected by an avalanche photodiode (APD). In this particular implementation an optical beam splitter is used to divert a portion of the source signal to the APD, thus providing the "start timer" mark for range determination. The accuracy of such systems depends on a number of factors including the pulse width, detector electronics bandwidth, and the processor implementation. If the APD bandwidth is $2 \mathrm{GHz}$ and the analog-to-digital 


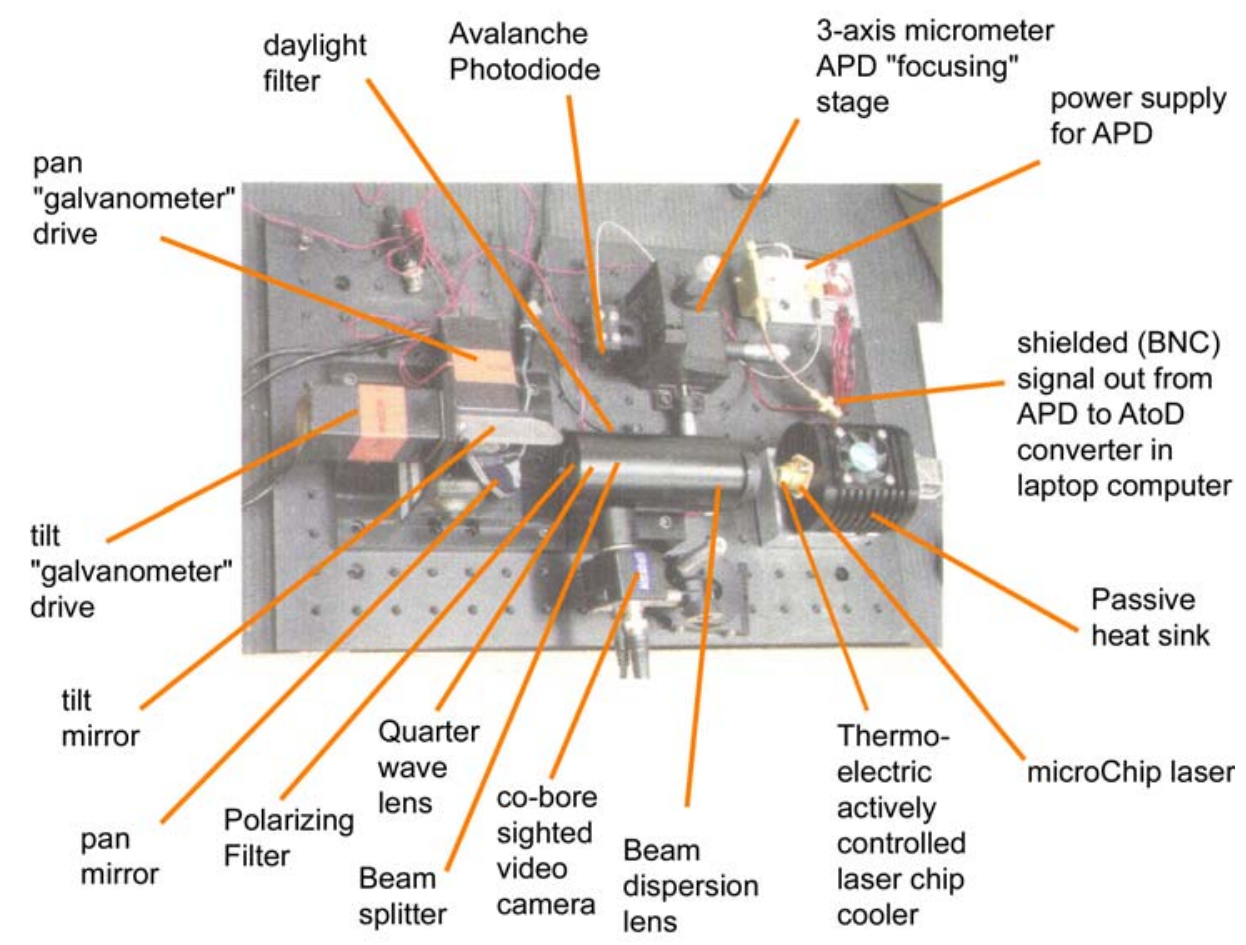

Figure 2.1.3: Typical physical implementation of a direct time-of-flight LADAR Scale is approximately $300 \mathrm{~mm}$ long by $150 \mathrm{~mm}$ wide (image courtesy Night Vision Lab).

readout (see ROIC) is matched, then the timing "bin" width is approximately $0.5 \mathrm{ns,} \mathrm{but}$ since that is round-trip, the range bin accuracy is thus $75 \mathrm{~mm}$. This 0-D ranging system is then scanned in 2D using electromechanically steered mirror systems.

\subsubsection{Limitations of Direct TOF}

Thus far we have made the assumption that all of the photons that are generated hit one specific object and are reflected back to the detector in a narrowly discriminating beam yielding one range measurement per pixel. This is not the case as shown in Figure 2.1.4.

\section{Uncompensated MicroChip Laser Beam Divergence}

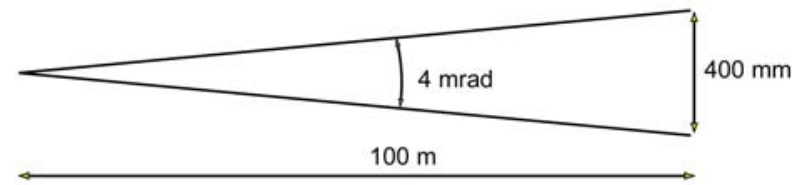

With Diffraction-Limited Optics
Figure 2.1.4: Source beam divergence variance and its effect on absolute beam diameter at $100 \mathrm{~m}$ range.

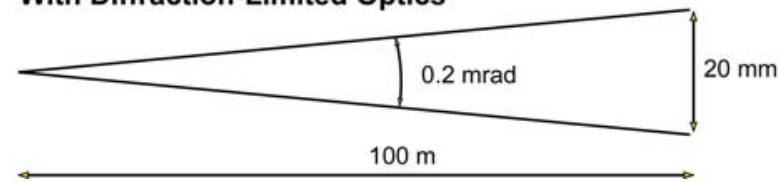


Due to imperfect optics and atmospheric dispersion the source illumination beam (pulse) expands with range; good industrial LADARS that have achieved near-diffraction limited optics have beam dispersion angles of around $0.2 \mathrm{mrad}$. Even so, this produces a finite beam diameter at $100 \mathrm{~m}$ of around $20 \mathrm{~mm}$. This has explicit physical consequences in the reported range measurement. Because the unambiguous range of the device shown in Figure 2.1.3 is on the order of $15 \mathrm{~km}$, one receives, in time, responses from photons from the illumination pulse arriving at different times related to

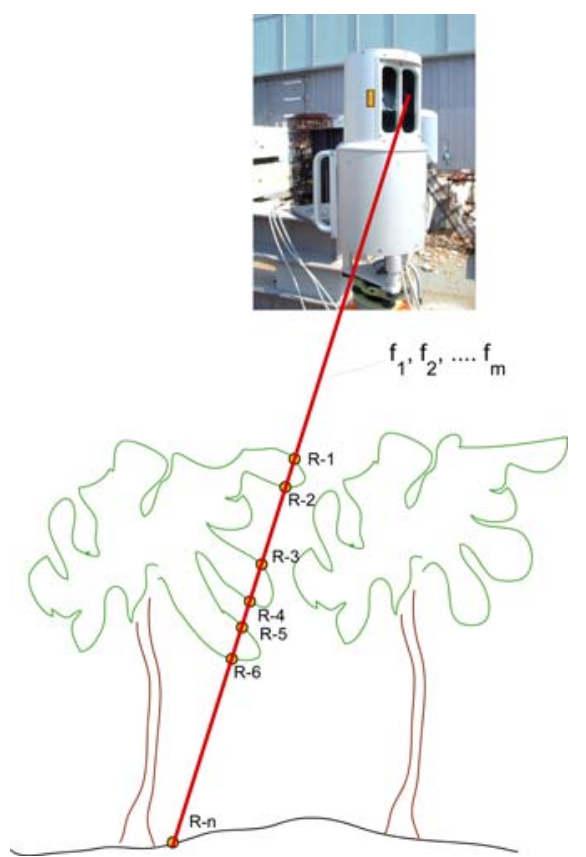

Figure 2.1.5: Due to beam divergence photons associated with a single "pixel" in a LADAR frame may represent significantly differing range data.

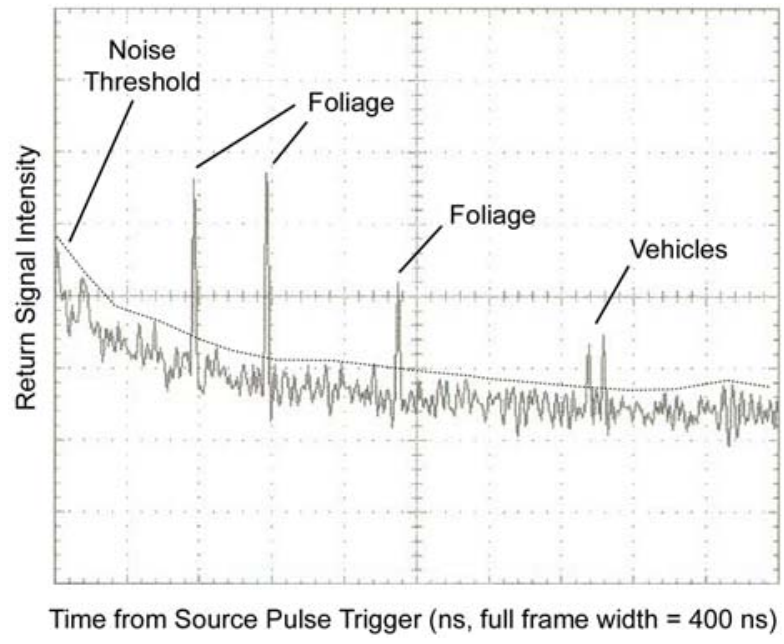

Figure 2.1.6: Full time-domain response for a 1ns pulse. Response was captured in 801 "range bins." The time-width of each bin was $0.5 \mathrm{~ns}$, or $150 \mathrm{~mm}$ (courtesy Night Vision Lab).

the different objects they hit within the cone of the dispersed beam. Figure 2.1.5 illustrates this point. A single "pixel" in the LADAR frame will in fact frequently have multiple valid ranges as the expanded beam penetrates the foliage. Figure 2.1.6 shows a time-domain response that might be anticipated from a system that was able to record the entire record of the received pulse over time. Any strong return above the noise threshold represents a valid object detection. Thus, one could store not one value, but a vector of values, for each pixel. Some LADARs now being developed illuminate at more than one wavelength. The response at each pixel can then be represented by a matrix with $n$ returns per source frequency $f$, as shown at right. 

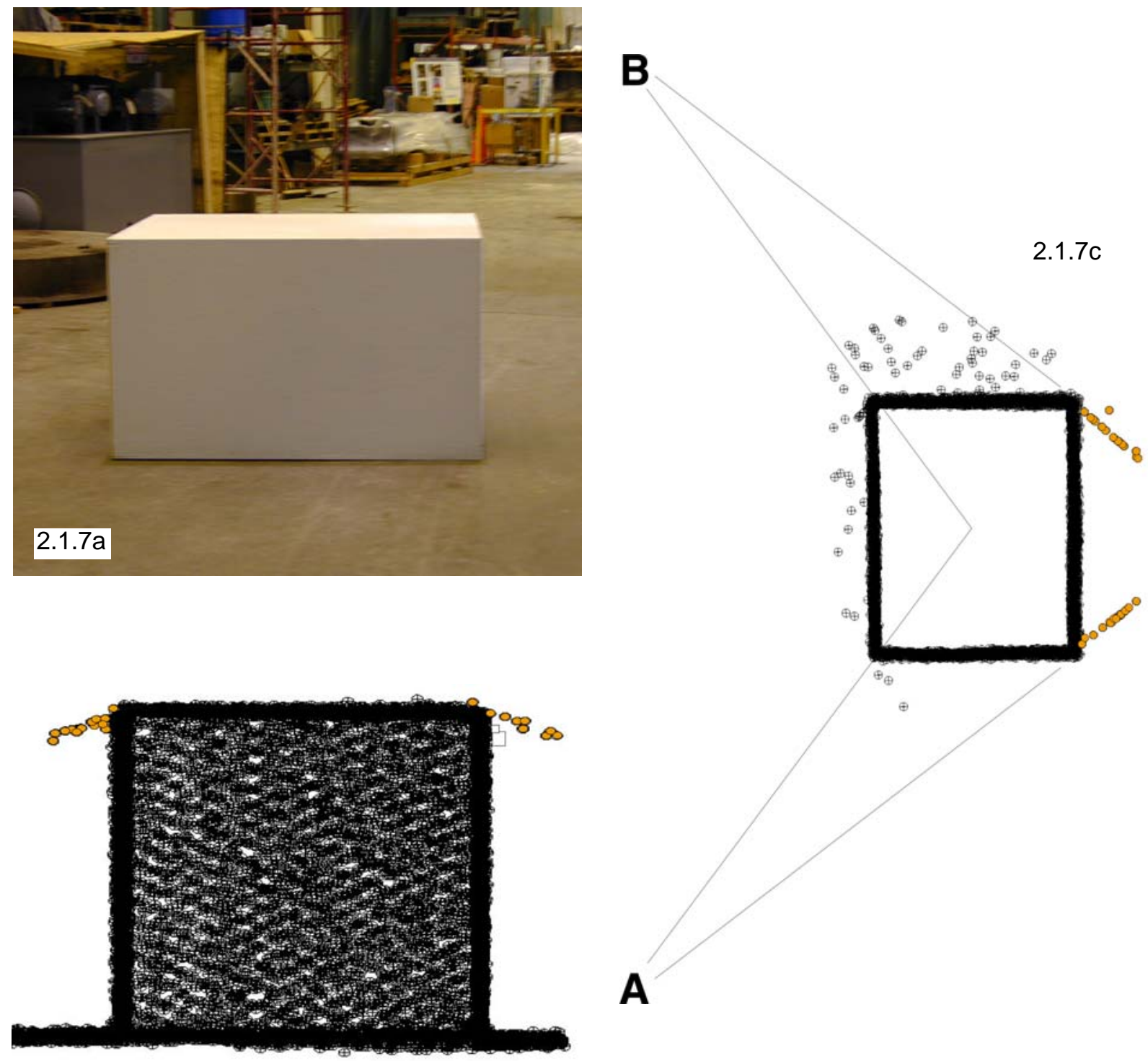

A

\subsection{7b}

Figure 2.1.7: Phantom Points Phenomenon. 2.1.7a (top left): Calibration block at NIST that was used for performance testing of LADAR point cloud registration and meshing algorithms. 2.1.7b (lower left) and 2.1.7c) (above right) illustrate the presence of phantom points formed along both the vertical edges (2.1.7c) and horizontal edges (2.1.7b) for instruments that report a single averaged range reading (as opposed to all valid returns within a pixel field of view). The block was modeled in 3-D using four registered scans at a reported accuracy of $+/-20 \mathrm{~mm}$; the thick black lines shown in Figures 2.1.7b) and 2.1.7c) are in fact composed of thousands of 3-D points and the composite graphically illustrates the variance on an otherwise planar surface. Individual scans were taken from viewpoints along radials at 3 $\mathrm{m}$ from the center of the block through the vertical corner edges. Two such viewpoints, " $A$ " and "B" in 2.1.7c are marked in the plan view of the block. The phantom points associated with the rear vertical edges are highlighted in orange. The un-colored 3-D point outliers in Figure 2.1.7c are shown in orange in the side view of Figure 2.1.7b (below, left). Some instrument makers are now providing software options to select either the first or last return to be recorded. In many cluttered environments, however, including construction, the advantages of an "N-return" per pixel system are significant. 


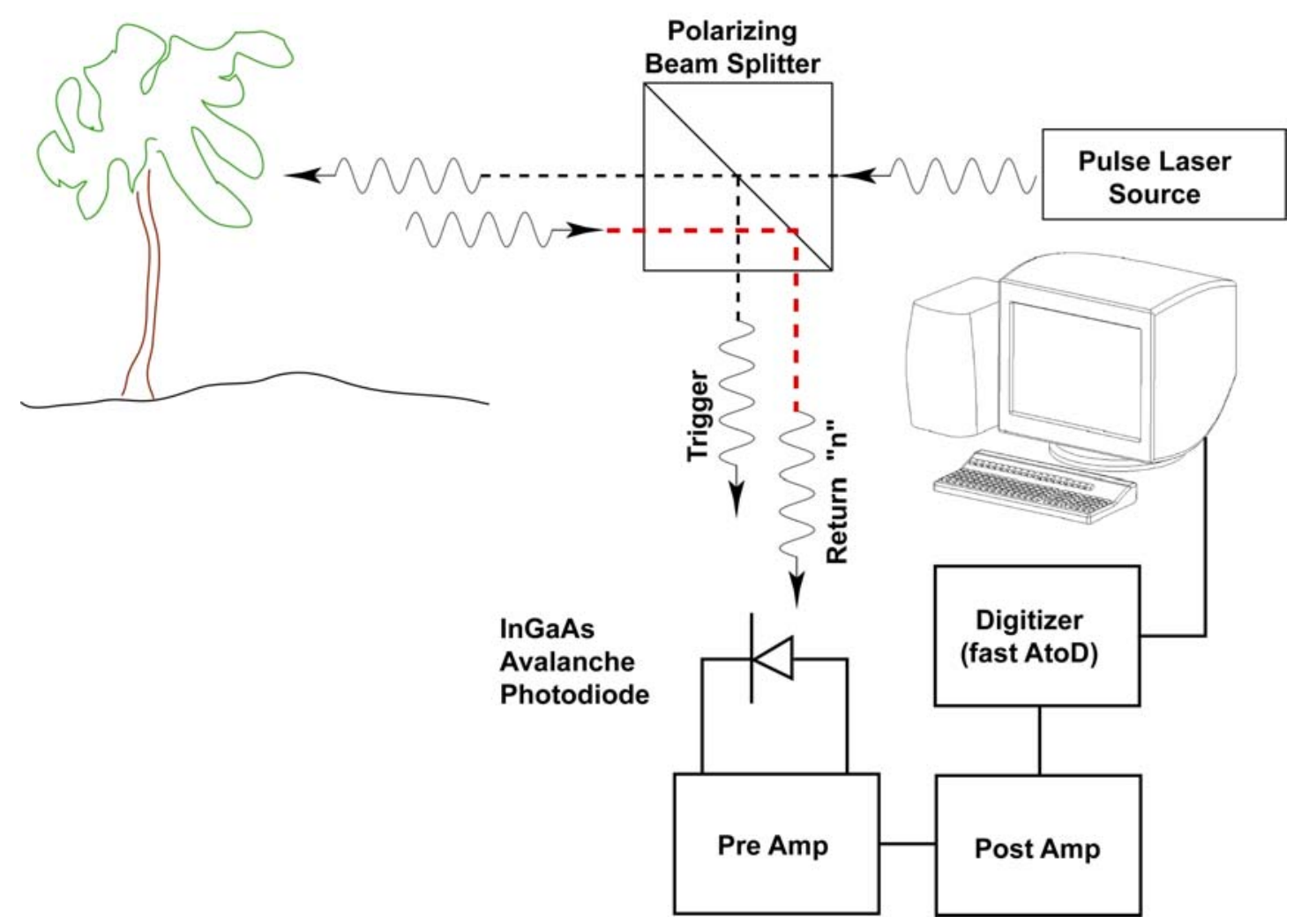

Figure 2.1.8: The achievable accuracy in a direct time-of-flight LADAR is controlled not only by the signal to noise ratio (SNR) but also by the bandwidth of the system at each component. Very fast APDs (running at approximately $10 \mathrm{GHz}$ ) are now available commercially through telecommunications component manufacturers; yet the fastest single channel digitizer can sample only $4^{\star} 10^{9}$ samples/second.

At present, no commercial pulse TOF LADAR provides the user with this kind of pixel response matrix (or even a single frequency time domain vector). Instead, it is common to average earlier arrivals that have a strong signal-to-noise radio (SNR) and report that as a single range per pixel. The results of this averaging are shown in Figure 2.1.7, where non-existent "phantom" points become part of the point cloud data set. The point here is that each of the valid returns shown in Figure 2.1.6 represent usable engineering information that is presently not available. The development of methods to achieve the acquisition of such "n-return" per pixel capability is considered a high priority for Next-Generation LADAR.

Recognizing this limitation, particularly in the presence of fog or dust, some LADAR manufacturers have begun offering "first" and "last" return options. If some of the outgoing photons manage to make a 2-way transit of the intervening medium (fog, dust, tree leaves) then the last return can be expected to represent "ground truth" -- the true range of a physical solid detected within the scene blurred by environmental effects. But this ignores the problem of multiple, valid targets within a pixel field of view, and this problem persists regardless of whether "first" or "last" returns are reported. It is a limitation of existing technology that not all valid returns above the noise threshold are reported. 
Pulse TOF systems, such as diagramatically shown in Figure 2.1.8, are limited in their accuracy not only by the bandwidth of the detector (currently limited to a maximum of approximately $10 \mathrm{GHz}^{*}$ ), although research in fiber optic tele-communications is competitive and is pushing this figure of merit higher), but also by the bandwidth of the post-detection conversion read-out circuitry. Presently, fast stand-alone single channel digitizers can achieve $4{ }^{\star} 10^{9}$ samples per second ${ }^{\star *}$ operating throughput. All other elements of the system notwithstanding, this immediately limits the best direct range resolution bin size to $250 \mathrm{ps}$ round trip $\left(1 / 4^{\star} 10^{9}=250^{\star} 10^{-12} \mathrm{~s}\right)$, or $125 \mathrm{ps}=75 \mathrm{~mm}$ for oneway distance to the target ( $\Delta \mathrm{d}$, following Eq. 2.1.2 below).

$$
\Delta d=\frac{c \cdot \Delta t}{2}
$$

Similarly, the pulse width of the source illumination affects the achievable accuracy, since edge detection is enhanced by a shorter, sharper pulse. The shortest pulse source currently used in an operational LADAR device is 250 ps [Aull et al, 2001], although this is by no means close to the shortest laser pulses thus far generated. More on this subject will be presented in Chapters 4 and 5 .

Desipite this apparent physics limitation, many commercial direct time-of-flight LADARs report range accuracies in the $10 \mathrm{~mm}$ to $20 \mathrm{~mm}$ range, not $75 \mathrm{~mm}$. Approaches to range accuracy improvement generally center around what are broadly referred to as "super resolution" algorithms [e.g. Cheeseman, 1994] originally developed for sharpening blurred video images and techniques for improving edge detection in the presence of noise.

Super resolution is the process of combining information from multiple low-resolution images to form a single high-resolution image. The Cheeseman algorithm for superresolution is based on a Bayesian framework that can be adapted to the analysis of several consecutive single pixel responses (e.g. the binned return signal output from a 4 Giga-sample/s digitizer) to produce a sub-bin-length estimate for the time-of-flight. Ultimately, the circuit speed of the digitizer will be limited by the responsivity of the semi-conductor materials.

*e.g. the SU020-10A-TO $10 \mathrm{GHz}$ Avalanche photodiode from Sensors Unlimited, Inc.; http://www.sensorsinc.com/product

**e.g. the Cougar 1010-4, 4 Giga-sample per second standalone V-Class Multichannel Digitizer, http://www.acqiris.com/products 


\subsection{Phase-Based AM-CW}

One means for improving accuracy over direct time-of-flight distance measurement is through the use of phase detection. This concept is illustrated in Figure 2.2.1. If the source is modulated at a single sinusoidal frequency, $f$, then a phase shift of

$$
\Delta \phi=2 \pi \mathrm{f}\left(\mathrm{t}_{\text {ROUNDTRIP }}\right)=2 \pi \mathrm{f}\left(\frac{2 \mathrm{~d}}{\mathrm{c}}\right)
$$

will be observed between the transmitted and received signal. Therefore, the object distance is given by:

$$
d=\frac{\Delta \phi c}{4 \pi f}
$$

The unambiguous range resolution is directly proportional to the source modulation frequency, $f$, while the accuracy is directly proportional to the SNR. The two are related; the smaller the unambiguous range for a given SNR the finer can be parsed the phase difference, thus improving the accuracy. For the case shown in Figure 2.2.1, the unambiguous range is half the wavelength at $f=20 \mathrm{MHz}$, or $7.5 \mathrm{~m}$.

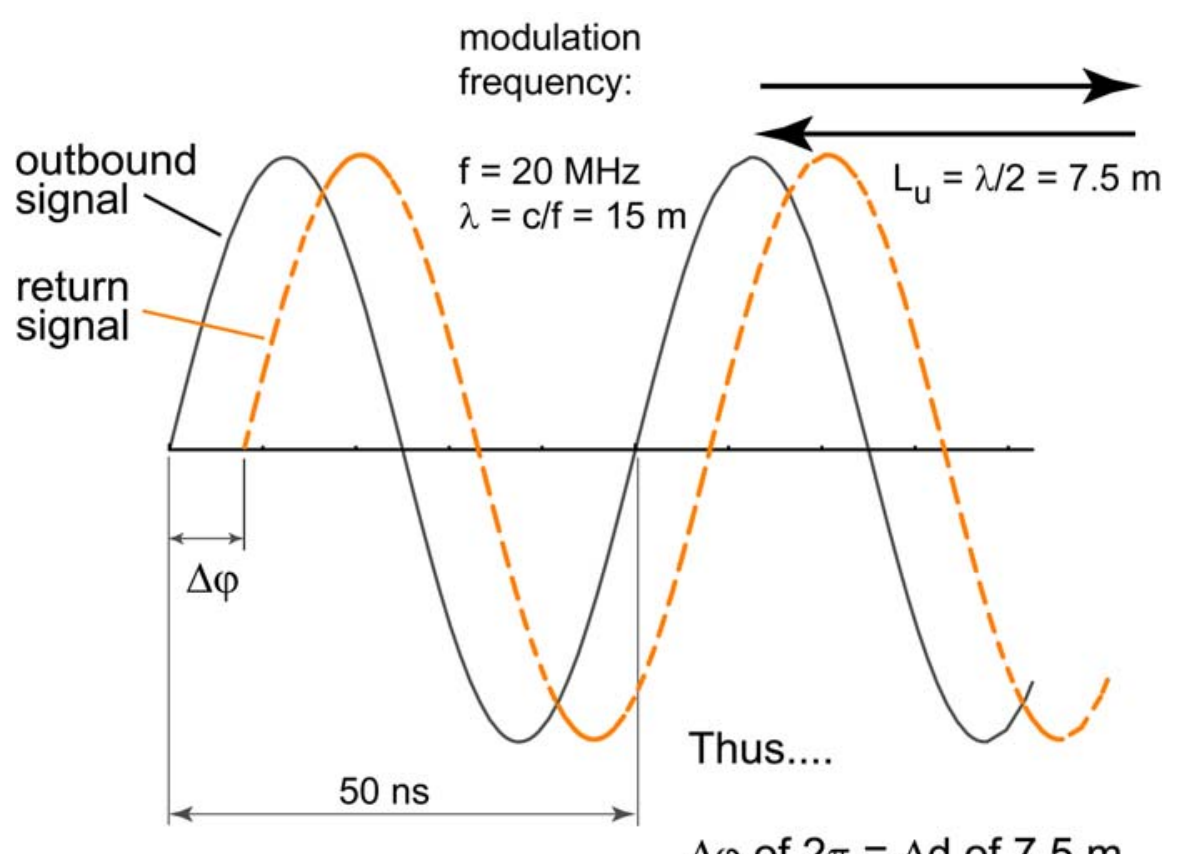

Figure 2.2.1: Phase-based determination of time-of-flight. Primary modulation frequency establishes the maximum unambiguous range for the device. 
SNR can be improved by integration over many cycles, but at the cost of raw throughput (frame rate). Both modulation frequency and SNR play a direct role in determining the range accuracy as per Eq 2.2.3:

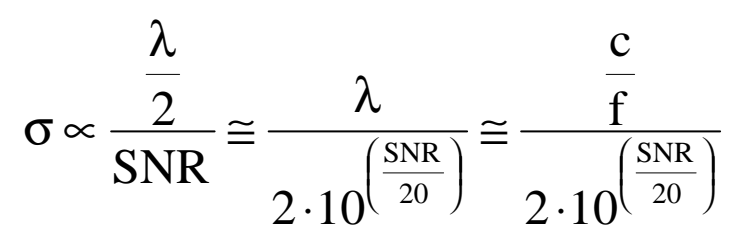

The SNR $(\mathrm{dB})$, refers to the measured signal read from the sensor and is defined as:

$$
\mathrm{SNR}=20 \cdot \log \frac{\mathrm{A}_{2}}{\mathrm{~A}_{1}}
$$

where $A_{2}$ is the measured signal amplitude and $A_{1}$ is the background noise amplitude. From an electronics standpoint Eq. 2.2.4 is means of quantifying how many more signal electrons are being produced at the sensor than noise electrons. More signal electrons, provided the digitizing system (e.g. an analog-to-digital converter) contains enough bits to resolve the count, equates to a finer parsing of the unambigous range and hence improved accuracy. Some practical solutions to how this is actually achieved in practice will be discussed later in this section.

The important lesson is that repeated sampling (often numbering in the thousands of samples), higher return light flux (both from using higher power sources and better optics), and higher efficiency photoelectron production in the sensing material will all improve the $\mathrm{S} / \mathrm{N}$ value and hence the accuracy for a phase-based measurement system. As an example then, for an AM/CW LADAR with a source modulation frequency of $20 \mathrm{MHz}$, and a measured SNR of $70 \mathrm{~dB}$, we find an approximate accuracy (thermal noise and other issues to be discussed later notwithstanding) of:

$$
\sigma \cong \frac{\frac{3 \cdot 10^{8} \mathrm{~m} / \mathrm{s}}{2 \cdot 10^{7} \text { cycles } / \mathrm{s}}}{2 \cdot 10^{\left(\frac{70}{20}\right)}}=2.37 \mathrm{~mm}
$$


There is a tradeoff to this equation. If you can receive greater power at the sensor you can shorten the integration time to achieve a desired level of accuracy. Conversely, a longer integration time collects more background radiation (noise). So the balance is to select a measurement time to where the SNR is as good as you need. As mentioned earlier if you raise the frequency, $f$, the non-ambiguous range grows shorter as the achievable measurement accuracy improves. There are several methods that have been developed to extend accuracy to greater distances including:

- FM-CW

- Pseudorandom Noise

- Use of second or multiple wavelengths

The latter technique is used in the commercial LADAR produced by $Z+F$ [Langer 2000].

The approach shown in Eq. 2.2.3 for estimating the accuracy of an AM-CW LADAR assumes that $100 \%$ of the signal modulation is transferred into the sensor material and converted to a usable signal. In actual practice, due to bipolar diffusion current, the modulation depth is less than 1. The more general form for the transmitted (modulated) and received signals is:

$$
\begin{aligned}
& I_{\text {modulation }}(t)=a_{2} \cdot \cos (\omega t) \\
& I_{\text {signal }}(t)=1+a_{1} \cdot \cos (\omega t-\phi)
\end{aligned}
$$

From this one may calculate the measurement uncertainty (standard deviation) as:

$$
\Delta d=\frac{\lambda \cdot \sqrt{2} \cdot \sqrt{1+\frac{\mathrm{K}^{2}}{2}}}{4 \cdot \pi \cdot \sqrt{\mathrm{N}} \cdot \mathrm{K} \cdot(\mathrm{SNR})}
$$

where $N$ is the phase shifting number (that is, the number of equally-spaced places the signal is sampled along a given wavelength), and $\lambda$ the modulation wavelength. $\mathrm{K}$ is known as the "modulation contrast" and is given by:

$$
K=\frac{a_{1} \cdot a_{2}}{2}
$$




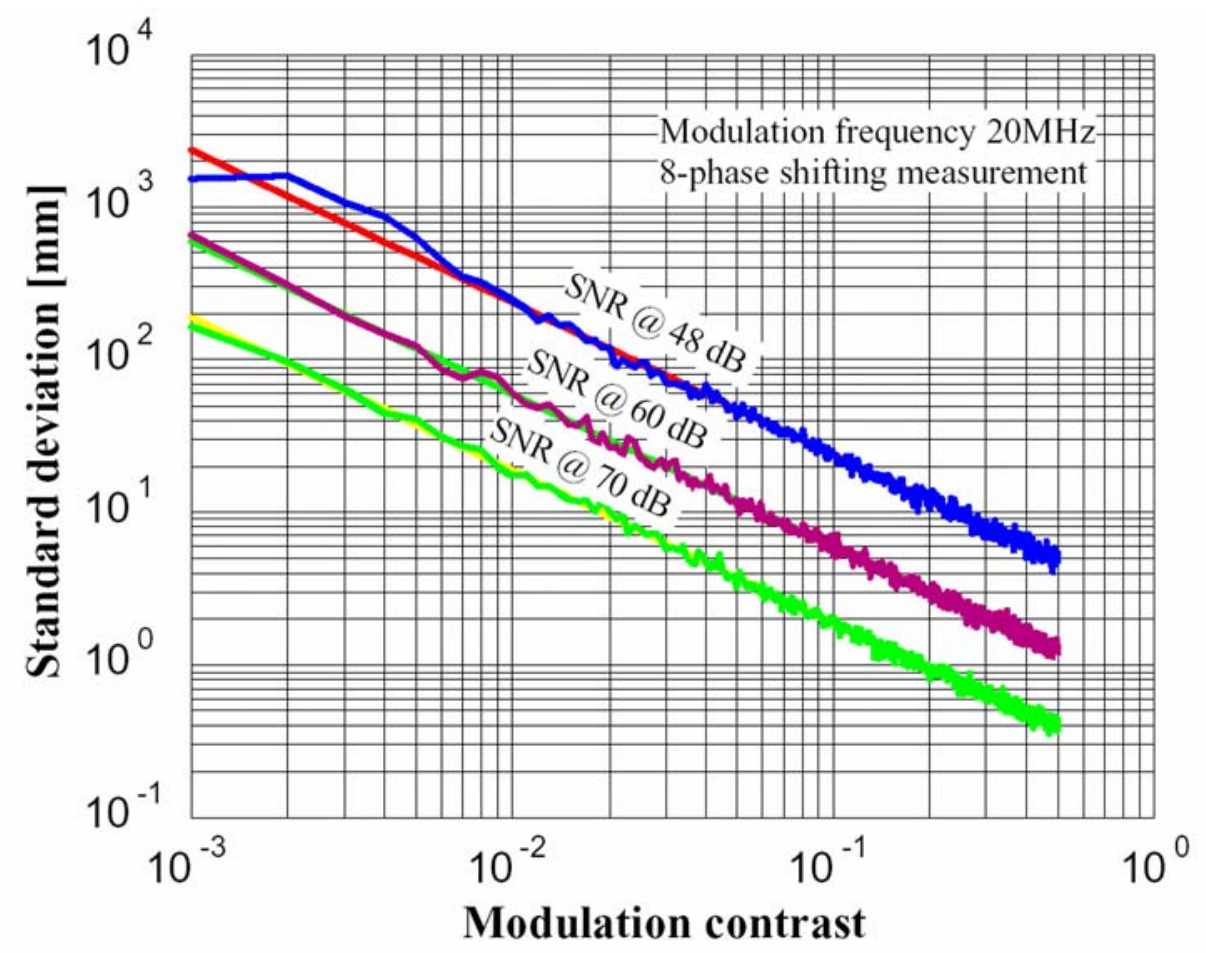

Figure 2.2.2: Accuracy of AM-CW Homodyne LADAR based on CMOS photonic mixer technology as a function of modulation contrast $(\mathrm{K})$ and SNR for a single modulation frequency of $20 \mathrm{MHz}$ and a sampling constant, $\mathrm{N}$, of 8 . [from Buxbaum, 2003].

Eq. 2.2.8 is plotted in Figure 2.2.2 and shows the achievable accuracy for an AMhomodyne LADAR. The modulation contrast, $\mathrm{K}$, is dependent on the characteristics of the semiconductor materials used in the detector and their efficiency at converting photoelectrons into measurable current that can be sensed; the SNR can be improved by any of the methods previously discussed. 


\subsubsection{Range Determination in AM-Homodyne LADAR}

Range determination using phase measurement is adapted from earlier work in radar in which the source and received signals are mixed and the phase, amplitude, and offset of the resulting signal are determined through solution of the resulting correlation function. The phase shift between the transmitted and received signals is determined by the 3D content of the illuminated scene and is the principal item of interest to be extracted from the mixed signal. Although there are a number of ways to do this, one of the most common is to synchronously demodulate the signal in the detector, through a process known as "photonic mixing."

The measurement of the cross-correlation function at specified phase locations allows the unknown phase of the return signal to be derived. The derivation presented below follows that of [Lange, 2000]. For an AM-CW LADAR the received optical signal will have the form:

$$
s(t)=1+a \cdot \cos (\omega t-\phi)
$$

where ' $a$ ' is the modulation amplitude, $\phi$ is the phase, $\omega$ the modulation frequency, and ' $\mathrm{t}$ ' the time. The source signal (used to demodulate the return signal) is given by:

$$
g(t)=\cos (\omega t)
$$

The correlation function is then given by:

$$
c(\tau)=s(t) \otimes g(t)
$$

Substituting Eqs 2.2.10 and 2.2.11 in Eq. 2.2.12 and expanding yields Eqs. 2.2.13 and 2.2.14 as:

$$
\begin{gathered}
c(\tau)=[1+\mathrm{a} \cdot \cos (\omega \mathrm{t}-\phi)] \otimes[\cos (\omega t)] \\
c(\tau)=\lim \mathrm{T}^{\prime} \rightarrow \infty\left\{\frac{1}{\mathrm{~T}^{\prime}} \int_{\frac{-\mathrm{T}^{\prime}}{2}}^{\frac{\mathrm{T}^{\prime}}{2}}[1+\mathrm{a} \cdot \cos (\omega \mathrm{t}-\phi)] \cdot[\cos (\omega \mathrm{t}+\omega \tau)] \mathrm{dt}\right\}
\end{gathered}
$$


from which a closed form solution is obtained:

$$
c(\tau)=\frac{a}{2} \cos (\phi+\omega \tau)
$$

In order to obtain a numerical solution for the amplitude, phase, and offset (DC signal component) one must evaluate the function at different phases, $\omega \tau$. Further, the measured signal will also include a component, denoted by the constant $b$ here, that represents the background radiation component (e.g. from sunlight). If the function is sampled at a spacing of $\pi / 2$ the following four equations result:

$$
\begin{aligned}
& c(0)=\frac{a}{2} \cos (\phi)+b \\
& c(1)=-\frac{a}{2} \sin (\phi)+b \\
& c(2)=-\frac{a}{2} \cos (\phi)+b \\
& c(3)=\frac{a}{2} \sin (\phi)+b
\end{aligned}
$$

The phase and amplitude of the incident signal is then obtained as:

$$
\begin{gathered}
\phi=\tan ^{-1}\left(\frac{c(3)-c(1)}{c(0)-c(2)}\right) \\
a=\frac{\sqrt{[c(3)-c(1)]^{2}+[c(0)-c(2)]^{2}}}{2}
\end{gathered}
$$


A more computationally flexible approach that lends itself to discretization as well as implementation in hardware [e.g. Buxbaum, 2002, Spirig, 1997] is to sample the modulated signal synchronously. If one uses $\mathrm{N}$ sampling points, the Nyquist criteria allows calculation of N/2-1 harmonics of the modulation frequency. Using the Discrete Fourier Transform

$$
\operatorname{DFT}\left(I_{n}\right)=\frac{1}{N}\left[\sum_{k=0}^{N-1} I_{k} \cdot e^{-i 2 k \frac{n}{N}}\right]=\frac{1}{N}\left[\sum_{k=0}^{N-1} I_{k} \cdot \cos \left(2 \pi k \frac{n}{N}\right)+i \cdot \sum_{k=0}^{N-1} I_{k} \cdot \sin \left(-2 \pi k \frac{n}{N}\right)\right]
$$

Eq. 2.2.19

One can then solve for the magnitude of the $\mathrm{n}^{\text {th }}$ coefficient as:

$$
A_{n}=\frac{1}{N} \sqrt{\left[\sum_{k=0}^{N-1} I_{k} \cdot \cos \left(2 \pi k \frac{n}{N}\right)\right]^{2}+\left[\sum_{k=0}^{N-1} I_{k} \cdot \sin \left(-2 \pi k \frac{n}{N}\right)\right]^{2}}
$$

The offset is:

$$
A_{0}=\frac{1}{N} \sum_{k=0}^{N-1} I_{k}
$$

And the phase of the $\mathrm{n}^{\text {th }}$ coefficient is:

$$
\phi_{n}=\tan ^{-1}\left(\frac{\sum_{k=0}^{N-1} I_{k} \cdot \sin \left(2 \pi k \frac{n}{N}\right)}{\sum_{k=0}^{N-1} I_{k} \cdot \cos \left(2 \pi k \frac{n}{N}\right)}\right)
$$

Several groups, including [Lange, 2000; Buxbaum, 2002; and Smithpeter et al 2000] have built operational AM-CW systems using a 4-sample $(\mathrm{N}=4)$ approach. This is illustrated graphically in Figure 2.2.3.

By using a single sinusoidal modulation frequency and by sampling the response at the detector at intervals of $\pi / 2$ (at four equally spaced temporal points) one can reduce Eqs 2.2.20 - 2.2.22 as follows for, respectively, phase, amplitude, and offset: 


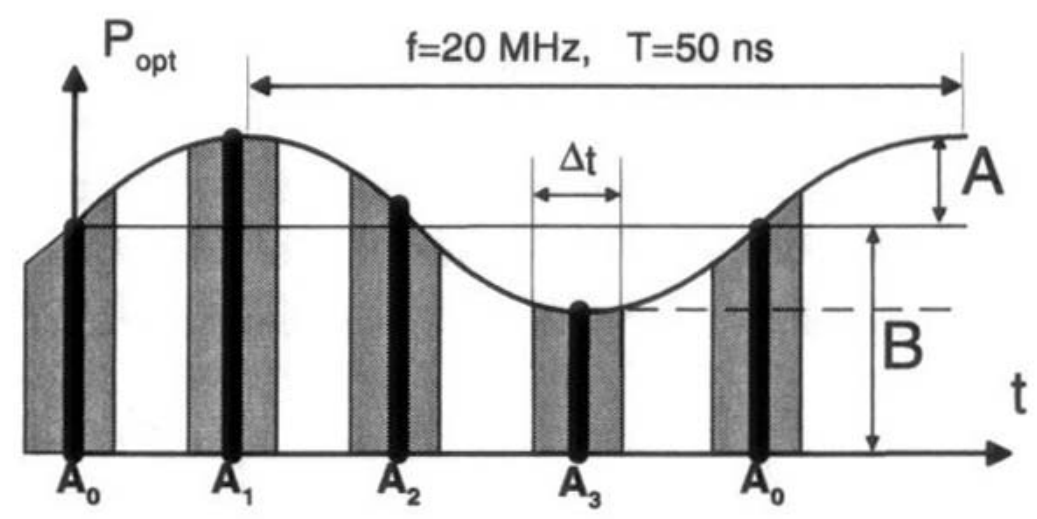

Figure 2.2.3: In a phase-based solution the transmitted and received signals are mixed. Discrete Fourier Transform (DFT) theory allows extraction of the phase, amplitude, and offset by sampling four points at an interval of $\pi / 2$ along the resulting waveform (from Lange, 2000)

$$
\begin{gathered}
\phi=\tan ^{-1}\left(\frac{A_{3}-A_{1}}{A_{0}-A_{2}}\right) \\
A=\frac{\delta}{2 \cdot \Delta t \cdot \sin \delta} \sqrt{\left[A_{3}-A_{1}\right]^{2}+\left[A_{0}-A_{2}\right]^{2}} \\
B=\frac{A_{0}+A_{1}+A_{2}+A_{3}}{4 \cdot \Delta t}
\end{gathered}
$$

where

$$
\delta=\frac{\pi \cdot \Delta t}{2}
$$

represents a signal attenuation factor that depends on the integration time period $\Delta t$. and $A_{0}, A_{1}, A_{2}, A_{3}$ represent the charge integration of the mixed signal over the intervals shown in Figure 2.2.3.

The the physical implementation of this technology can take several forms, some of which will be discussed in greater detail later in this report. One now-common approach makes use of so-called photonic mixing (Figure 2.2.4) in which a standard CMOS photo diode is reverse biased by the same frequency source that modulates the transmitted illumination signal. This diode responds directly to the mixed signal consisting of the modulated bias and the incoming photoelectrons (which have been delayed, and hence phase-shifted to provide information on the 3D scene they illumi- 

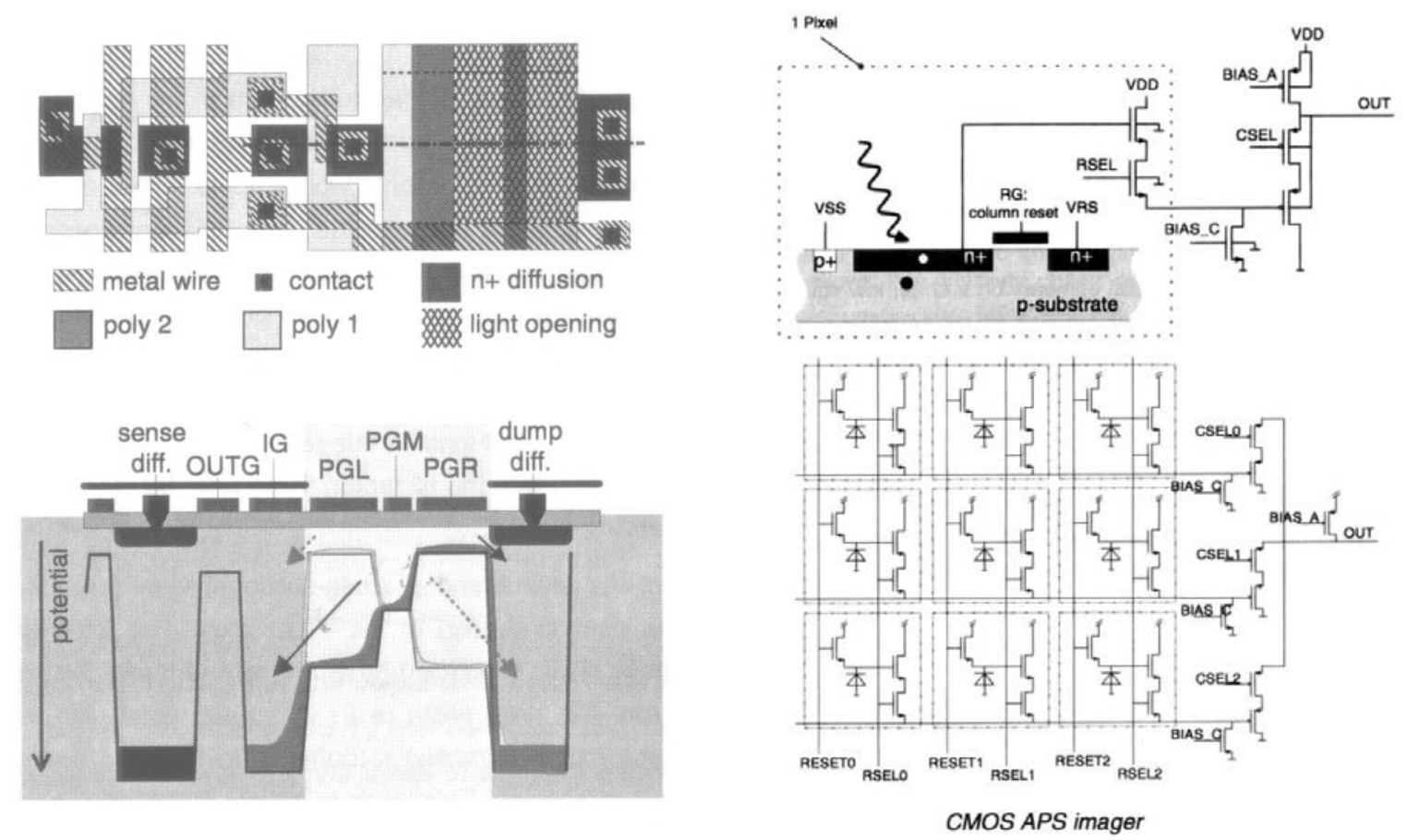

Figure 2.2.4: (a) (left): Example of an "Active Pixel Sensor," (APS) one of many versions of a Focal Plane Array (FPA) that can be used to simultaneously sample hundreds to thousands of pixels. (b) (right): a Focal Plane Array comprised of a matrix of APS sensors. FPAs form the core of "flash" LADAR. (from Lange, 2000, and Spirig, 1997).

nate). This technique is known as an AM-Homodyne LADAR. Timing circuitry causes the charge integrated over the white portions of the curve in Figure 2.2.3 to ground while summing (through a capacitor) the regions $A_{0}, A_{1}, A_{2}, A_{3}$, respectively, and selectively storing those values for later processing, either on-chip or offboard using Eq. 2.2.23. Figure 2.2.4a illustrates the process schematically and is the basis of two of the LADAR systems shown in Appendix A produced by CSEM and PMD. Figure 2.2.4b shows the electrical schematic of the connection between the CSEM CMOS photogate and the ROIC.

It is possible to implement 2D detectors using all CMOS fabrication technology in the form of Focal Plane Arrays (FPAs) such as that depicted schematically in Figure 2.2.4b. Individual pixels within a LADAR field of view are mapped (optically) to pixels on the FPA. Similar FPAs can be constructed to work on the the direct time-of-flight principle described earlier. Both approaches are referred to as flash LADAR. 


\subsubsection{Limitations of AM Phase Measurements}

Pure homodyne (single frequency, non-coherent) LADARs suffers from two significant limitations. As the modulation frequency is increased, thereby improving potential accuracy, the un-ambiguous range is reduced -- leading to aliasing or false targets if the range happens to be greater than the unambiguous range. A solution to this problem is to use multiple frequencies [4] in which a lower frequency signal is used to establish an un-ambiguous interval over a longer distance within which the higher frequency response is located. The mathematics for solving this approach are not significantly more complicated than for the pure homodyne solution, but it has yet to be implemented in silicon; a commercial 2-frequency LADAR is produced by $Z+F$ (see Appendix A).

A much more serious problem with phase-based measurement is illustrated in Figure 2.2.5. Due to beam divergence and varying reflectivity of target surfaces, both accuracy and resolution are affected for all types of LADAR, but particularly for AM-homodyne class devices which in effect integrate the reflected photons from all surfaces that are illuminated within the pixel FOV, thereby producing an erroneous average range. This error can be great, particularly if the targets are at substantial range and where either multiple discrete targets exist (e.g. shooting through foliage) or when a fully-resolved target has sharply inclined facets. The effect is, in fact, more serious than that for direct TOF measurement devices because the result is based on the presumption that the return photoelectron flux is directly proportional to the range, which it most definite-

\section{Range Resolution \& Accuracy}

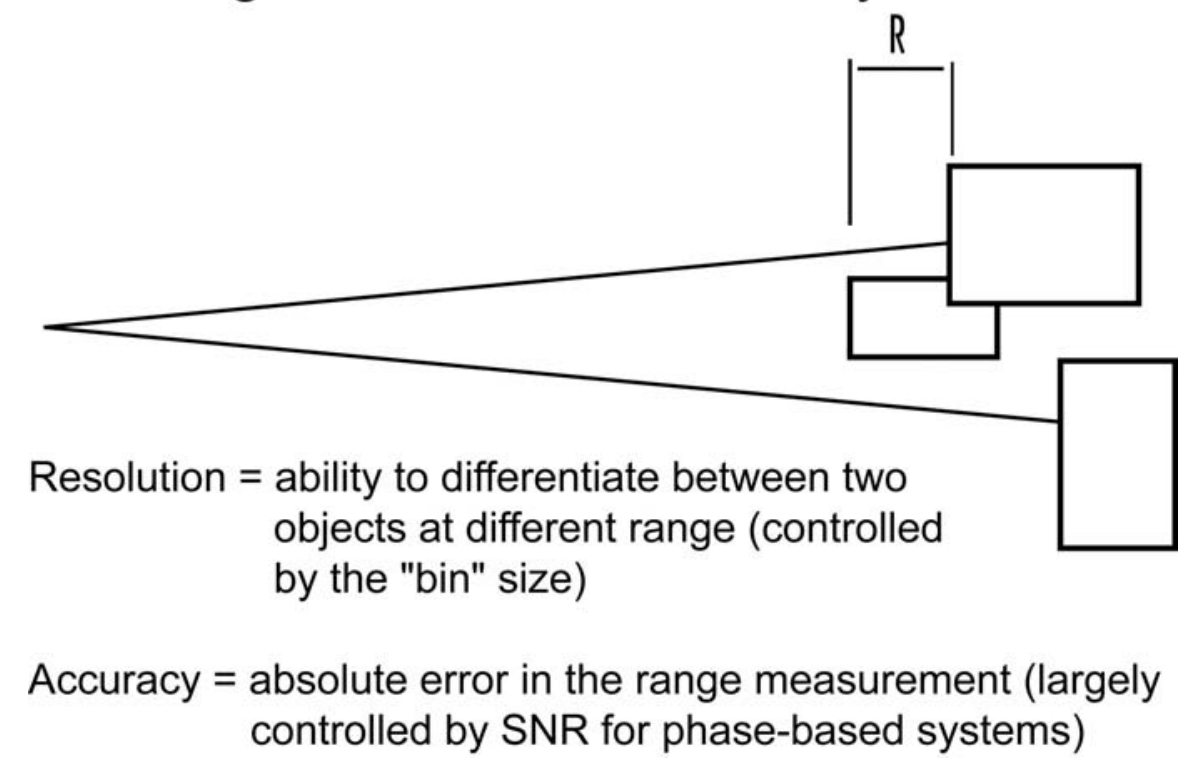

Figure 2.2.5: Due to beam divergence and varying reflectivity of target surfaces, both accuracy and resolution are affected for all types of LADAR, but particularly for AM-homodyne class devices which in effect integrate the reflected photons from all surfaces that are illuminated, thereby producing an erroneous average range. 
ly is not in many practical cases. For example, if the lower right block in Figure 2.2.5 happened to be coated with a retroreflective material (or even simply a more reflective material than the blocks in front) the resulting range reported would be longer, and erroneously biased towards the front surface of that block. The range is in effect a weighted average of the returns from all of the objects illuminated within a particular pixel. This, in turn, leads to the same type of phantom points described earlier. 


\subsection{Chirped AM-CW}

It is possible to recover multiple ranges per pixel in an amplitude modulated LADAR by producing a very specifically-shaped amplitude modulation, known as a chirp. The theory for such an approach was initially developed for radar [Skolnik, 1980] but only recently has been adapted to optical systems [e.g. Stann et al, 1996]. In a chirp pulse the frequency is varied linearly with time (see Figure 2.3.1). Experimental LADAR breadboards have been built where the start frequency of the chirp is $200 \mathrm{MHz}$ and the end frequency is $800 \mathrm{MHz}$. The start and stop frequencies are limited by the modulation bandwidth of the laser source and the bandwidth of the detector. The range resolution (per pixel) in such a LADAR is given as:

$$
\Delta R=\frac{c}{2 \Delta F}
$$

in which $\Delta \mathrm{F}$ is the the difference $(\mathrm{Hz})$ between the start and stop frequencies. For $\Delta \mathrm{F}$ of $600 \mathrm{MHz} \Delta \mathrm{R}=250 \mathrm{~mm}$. There are several methods for modulating the illumination source. These include amplitude modulation of the laser power (incoherent LADARs);
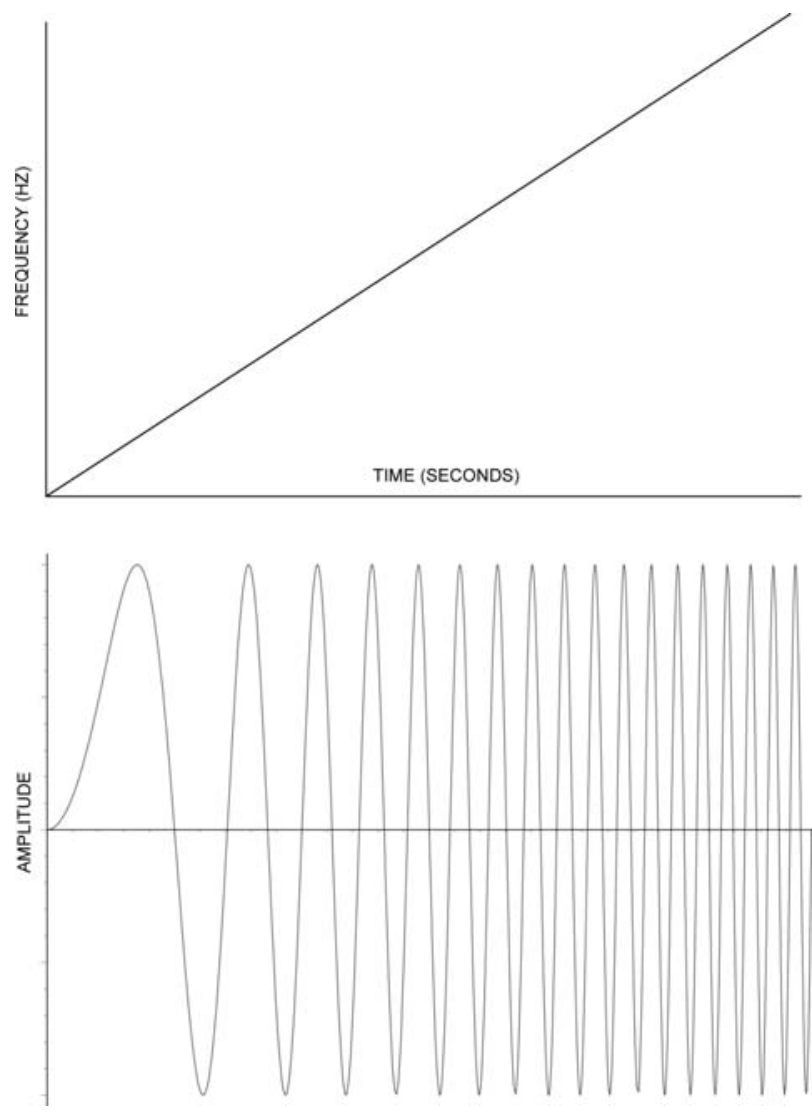

Figure 2.3.1: Linear Chirp. Frequency vs Time plot (top); Amplitude vs Time (bottom). direct frequency modulation of the laser; and phase modulation of the source (coherent LADARs), with each approach following the general prescribed variance with time as shown in Figure 2.3.1. For simplicity of exposition we follow [Stann et al] in this section and describe only the first method, that of modulating the amplitude of the source laser. This method is technically referred to as chirped AM/CW modulation. True frequency modulation (in which the actual frequency of the laser is varied over time) typically requires changing the optical cavity length of the laser, either by mechanical (e.g. piezo) or electro-optical means. Precision oscillators are required in both cases. Commercial devices using monolithic microwave integrated circuits are available for this purpose that are suitable for short range applications such as envisioned for NGL.

In a chirped $\mathrm{AM} / \mathrm{CW}$ system the chirp signal is fed into a wideband power 


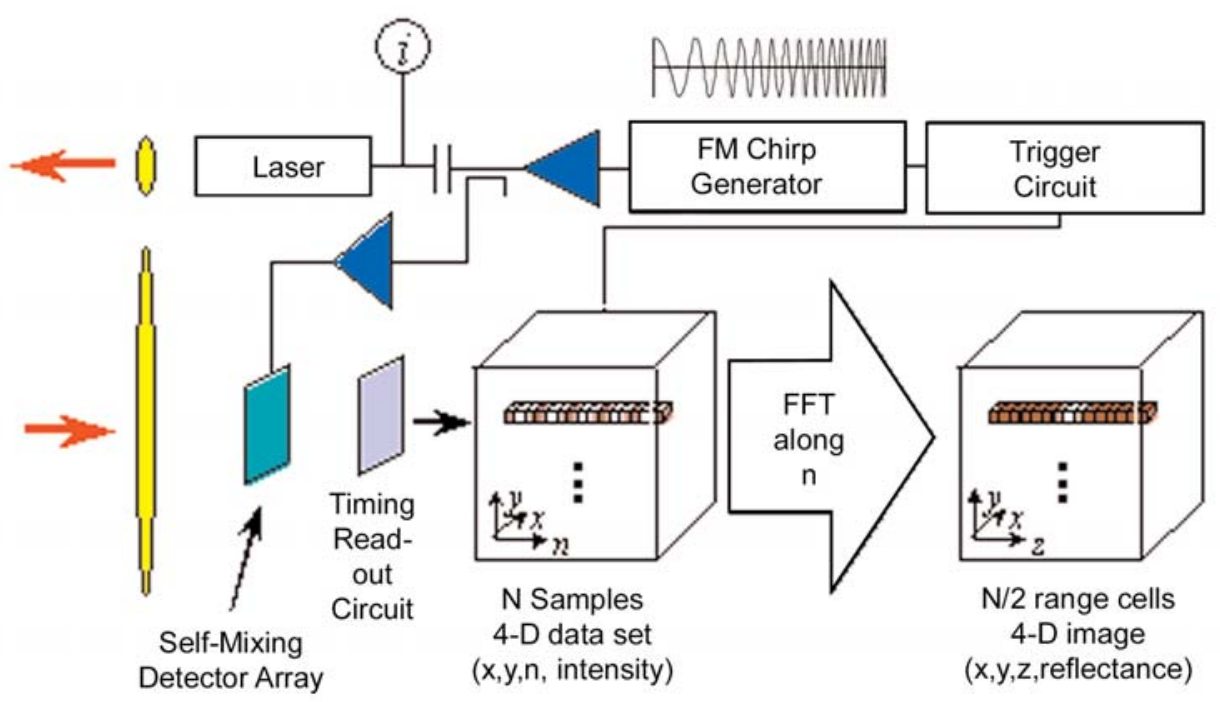

Figure 2.3.2: Block diagram for an experimental single channel (2-D) chirped-AM/cw LADAR. [courtesy ARL].

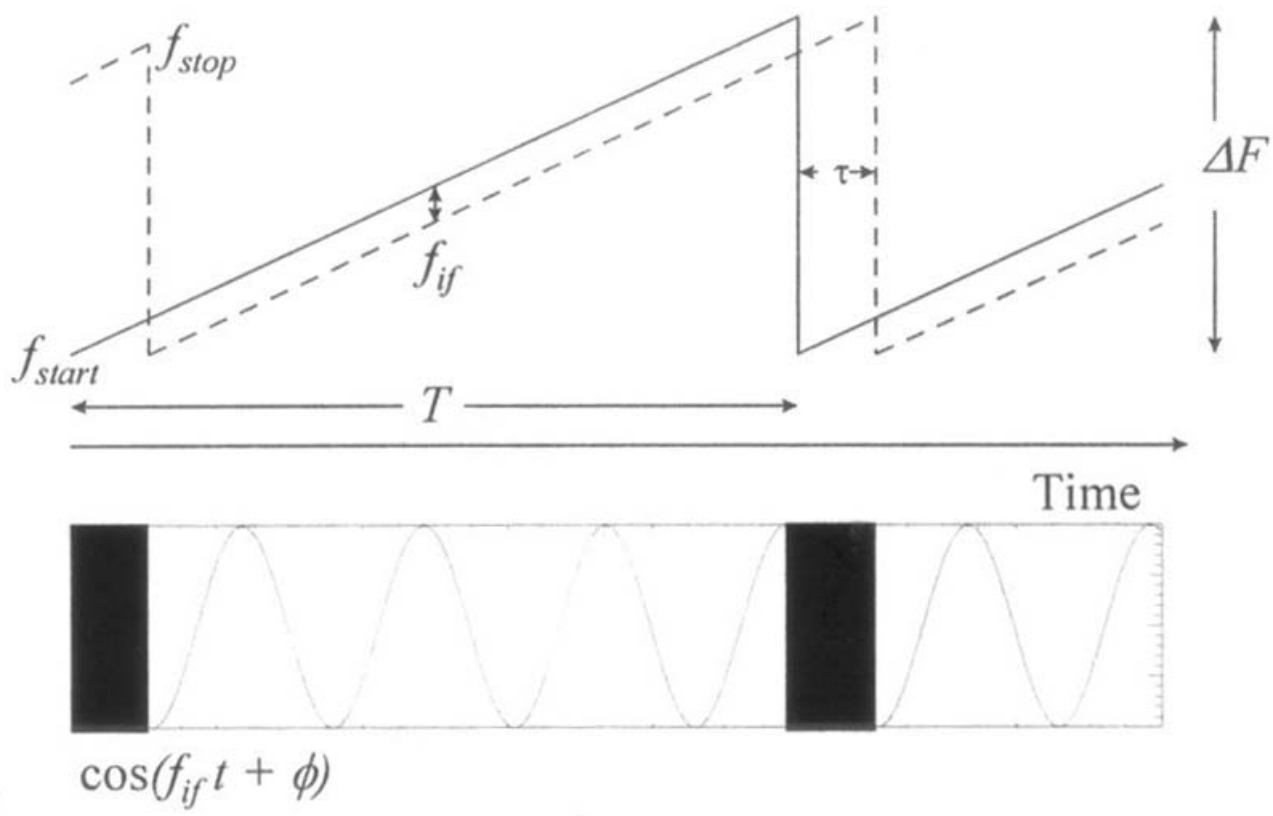

Figure 2.3.3: Chirped AM/CW ranging. A self-mixing detector is located at the focal plane of the receiver optics. The transmitter modulation waveform (local oscillator) is applied across the detector and the resulting photo-current is the product of the local oscillator waveform and that of the return signal. The transmitted and received waveform (dashed line) differ by the the intermediate frequency $\mathrm{f}_{\mathrm{if}}$, which can be sampled by the read-out circuit. [courtesy ARL]. 
amplifier that modulates the current driving a semiconductor laser diode. A circuit between the power amplifier and the laser diode matches the driving impedance of the amplifier to the laser diode impedence over $\Delta \mathrm{F}$. This causes the light beam intensity to modulate over the chirp bandwidth. The laser diode characteristics limit the achievable modulation bandwidth of the system and hence the achievable accuracy.

The laser diode converts the chirp current waveform into a light waveform where the power is proportional to the driving current, hence the name AM/CW (Amplitude-modulated, continuous wave). The return light signal enters an array of self-mixing custom metal-semiconductor-metal (MSM) detector that is biased by a portion of the signal coming from the RF amplifier that drives the laser, thus producing a similar electronic effect to the previously described photonic mixing technology for single range measurement AM/CW LADARs. Strictly speaking this is electronic, not optical, mixing of the two signals.

Because the optical return signal is mixed electronically with the source modulation signal (as opposed, e.g., to coherent mixing discussed below) the bandwidth of the detector is of critical importance in high resolution designs. Commercial telecommunications photodiodes (e.g. APDs and MSMs) have bandwidths ranging from $1 \mathrm{GHz}$ to $10 \mathrm{GHz}$ and similarly limit one-way range resolution to $300 \mathrm{~mm}($ at $1 \mathrm{GHz})$ and $30 \mathrm{~mm}$ (at $10 \mathrm{GHz}$ ). In the approach taken by Stann et al the current from the MSM photodiode is converted into a voltage with a transimpedence amplifier. The system shown in Figure 2.3.2 consists of an MSM array and CMOS read-out circuit. The result is a sinusoidal signal whose frequency is proportional to target range and whose amplitude is proportional to target reflectivity. This signal must be subsequently processed, using an FFT, to obtain a time domain response.

Stann et al (1996) describes the mathematics of FM ranging theory as follows. The frequency of the transmitted waveform is given by:

$$
f(t)=f_{0}+\frac{\Delta F}{T} \cdot t, \quad-\frac{T}{2} \leq t \leq \frac{T}{2}
$$

where $f_{0}$ is the center or carrier frequency of the chirp waveform, $t$ is the time, and $T$ is the total time to complete one chirp (or chirp duration). 
The phase of the transmitted chirp waveform is given by:

$$
\phi_{X}(t)=\int_{-\infty}^{t} 2 \pi\left(f_{0}+\frac{\Delta F}{T} \cdot t^{\prime}\right) d t^{\prime}
$$

and the received signal phase is:

$$
\phi_{R}(t)=\int_{-\infty}^{t-\tau} 2 \pi\left(f_{0}+\frac{\Delta F}{T} \cdot t^{\prime}\right) d t^{\prime}
$$

The term $\tau$ in Eq. 2.3.4 is the round trip flight time from the source to target to detector, in seconds. The difference in phase between the received and transmitted signal is:

$$
\Delta \phi(t)=\phi_{X}(t)-\phi_{R}(t)
$$

Substituting Eqs. 2.3.3 and 2.3.4 yields:

$$
\Delta \phi(t)=\int_{(t-\tau)}^{t} 2 \pi\left(f_{0}+\frac{\Delta F}{T} \cdot t^{\prime}\right) d t^{\prime}
$$

This can be solved to give:

$$
\Delta \phi(t)=2 \pi\left(f_{0} t+\frac{\Delta F}{2 T} \cdot t^{2}\right)-2 \pi\left(f_{0}(t-\tau)+\frac{\Delta F}{2 T} \cdot\left(t^{2}-2 t \tau+\tau^{2}\right)\right.
$$

Noting that $\mathrm{T}$ (the chirp length) can be designed to be much greater than $\tau$, Eq. 2.3.7 can be simplified to obtain:

$$
\Delta \phi(t)=2 \pi f_{0} \tau+2 \pi \frac{\Delta F}{T} \tau t
$$


The intermediate frequency (IF) waveform can be shown as:

$$
V_{M}(t)=a_{R} \cos \left[2 \pi \tau\left(f_{0}+\frac{\Delta F}{T} t\right)\right]
$$

where $a_{R}$ is the amplitude of the received signal, accounting for all propagation, scattering, detection, and mixing losses and amplifier gains. The first term in Eq. 2.3.9 is a fixed Doppler phase term that is proportional to the carrier frequency and time delay or distance to the target. The second term represents a frequency proportional to the distance to the target.

The range can be determined by measuring the frequency of $V_{m}(t)$ over $T$. This can be accomplished through computation of the Fourier transform of the waveform as:

$$
F(f)=\frac{a_{R}}{T} \int_{-T / 2}^{T / 2} \cos \left[2 \pi \tau\left(f_{0}+\frac{\Delta F}{T} t\right)\right] e^{-j 2 \pi f t} d t
$$

Integration of Eq. 2.3.10 yields:

$$
F(f)=e^{j 2 \pi f_{0} \tau} \cdot \frac{\sin \left[\pi\left(\Delta F \frac{\tau}{T}-f\right) T\right]}{\pi\left(\Delta F \frac{\tau}{T}-f\right) T}+e^{-j 2 \pi f_{0} \tau} \cdot \frac{\sin \left[\pi\left(\Delta F \frac{\tau}{T}+f\right) T\right]}{\pi\left(\Delta F \frac{\tau}{T}+f\right) T}
$$

Eq. 2.3.11

In Eq. 2.3.11 the term $a_{R}$ has been removed to normalize the response. For a LADAR architecture employing the discrete Fourier transform to obtain range cells, each complex output sample of the transform represents the magnitude and phase of the respective harmonic frequency components of the input waveform that occur at integer multiples of $1 / \mathrm{T}$. This allows $f$ to be replaced in Eq. 2.3.11 with $\mathrm{n} / \mathrm{T}$ as follows:

$$
F\left(\frac{n}{T}\right)=e^{j 2 \pi f_{0} \tau} \cdot \frac{\sin [\pi(\Delta F \tau-n)]}{\pi(\Delta F \tau-n)}+e^{-j 2 \pi f_{0} \tau} \cdot \frac{\sin [\pi(\Delta F \tau+n)]}{\pi(\Delta F \tau+n)}
$$

Eq. 2.3.12 
The exponential factors in Eq. 2.3.12 represent the Doppler component present in each harmonic line, while the $(\sin x) / x$ factors represent the amplitude of the Doppler components. For $\mathrm{n}>4$ the second term can be ignored. The harmonic component of $\mathrm{F}(\mathrm{n} / \mathrm{T})$ in the first part has a maximum for:$$
\Delta F \tau=n
$$

Eq. 2.3.13

This means that the output samples of the discrete fourier transform have been mapped to normalized range. Thus, each output sample corresponds to a range gate. The system response, for $n>4$ approaches that of a sync function whose maxima is centered on an integer value of normalized range. The spacing is uniform and integer which means that:

\section{$\Delta F \Delta \tau=1$}

However, from a variant of Eq. 2.1.1:

$$
\Delta \tau==\frac{2 \Delta R}{c}
$$

and thus, the range resolution of this class of system is given by:

$$
\Delta R=\frac{C}{2 \Delta F}
$$

Which confirms Eq. 2.3.1. Experimental and numerical simulations conducted by [Stann et al.,1996] verify that the above theory is applicable for situations where $\Delta \mathrm{F}$ is a high percentage of $\mathrm{f}_{0}$, which would be the case for the design of high resolution LADARs.

The chief limitations of the AM-CW LADAR approach are the need for additional frontend hardware (complicated chirp-generation electronics that add both cost, size, and an additional level of noise), the frequency response of the laser source (which may be bandwidth limited); and the manifold increased mathematical post-processing that is involved for real-time performance. 
For a typical laser diode with a bandwidth of $2 \mathrm{GHz}$ the achievable range resolution ("bin" or "gate" width at the detector) is $75 \mathrm{~mm}$ round trip. From this it becomes immediately clear that for a system being designed for a range resolution of several $\mathrm{mm}$ that the source and detector bandwidth will be the problem forcing itself upon our attention. This physics limitation applies to both pulsed and chirped systems. 


\subsection{Coherent LADAR}

The terms coherent and non-coherent have specific architectural connotations in LADAR literature. In coherent detection (also known as heterodyne) the return signal from the target is optically mixed with a reference laser, known as a local oscillator or "LO" - commonly a highly stable continuous wave laser. The two mixed signals, which must be wavefront matched and combined through a heterodyne mixer, are focused onto a photodetector (e.g. an APD or PIN chip). A simplified schematic of how this might be implemented is shown in Figure 2.4.1. Other examples are given later from implemented commercial products.

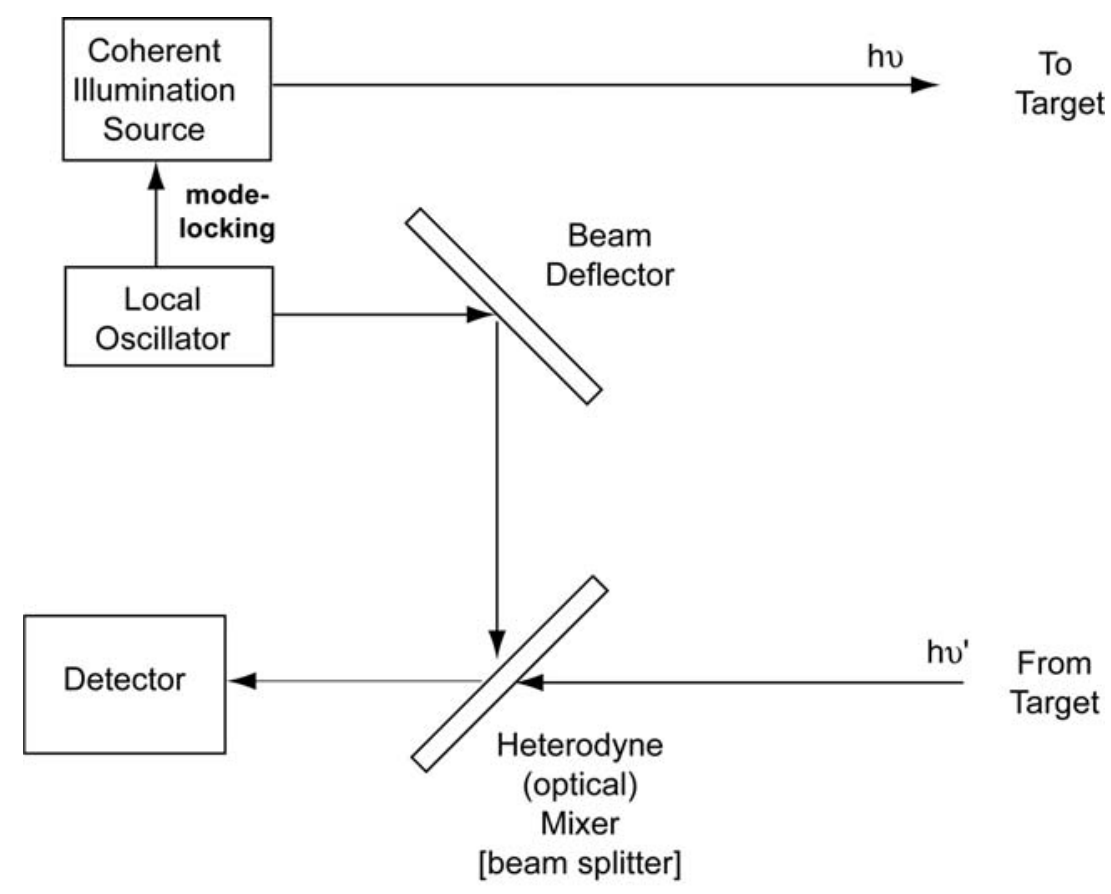

Figure 2.4.1: Simplified schematic of a coherent laser radar. A highly precise local oscillator (LO) is used to synchronize the continuous wave laser illumination source as well as to illuminate the heterodyne mixer. Although the LO and the coherent illumination source can be one and the same physical device it is more common to have a physically separate LO and to use mode-locking to synchronize the illumination source. In this manner the LO power impinging on the heterodyne mixer can be arbitrarily boosted to a level just short of saturation. Such techniques can be used to produce a shot noise dominated response that is effectively insensitive to ambient radiation sources (e.g. sunlight).

The intensity (in $\mathrm{W} / \mathrm{m}^{2}$ ) response at the detector is given by [Kamerman, 1996] as:

$$
I(t)=c \varepsilon_{0}\left[E_{L O}^{2}+E_{S}^{2}+2 E_{L O} E_{S} \cos \left(\left(\omega_{S}-\omega_{L O}\right) t\right)\right] \quad \text { Eq. 2.4.1 }
$$

Where 
$I(t)=$ intensity incident on the detector in $\mathrm{W} / \mathrm{m} 2$

$E_{\mathrm{LO}}=$ local oscillator electric field amplitude in $\mathrm{V} / \mathrm{m}$

$E_{s}=$ received signal electric field amplitude in $\mathrm{V} / \mathrm{m}$

$\omega_{\mathrm{s}}=$ received signal electric field frequency in $\mathrm{Hz}$

$\omega_{\mathrm{LO}}=$ local oscillator signal electric field frequency in $\mathrm{Hz}$

$\mathrm{c}=$ speed of light in a vacuum

$\varepsilon_{0}=$ electric permittivity of free space

The power (spatial integral of the intensity) on the detector is given by [Gatt, 2003] as:

$$
P_{\text {det ector }}=P_{L O}+P_{S}+2 \sqrt{\gamma P_{L O} P_{S}} \cos (2 \pi \Delta f t+\theta) \quad \text { Eq. } 2.4 .2
$$

where

$\mathrm{P}_{\mathrm{LO}}=$ integral of $\quad c \varepsilon_{0}\left|E_{L O}\right|^{2} \quad$ over detector area

$\mathrm{P}_{\mathrm{S}}=$ integral of $\quad \mathrm{c} \varepsilon_{0}\left|E_{S}\right|^{2} \quad$ over detector area

$2 \pi \Delta \mathrm{f}=\omega_{\mathrm{S}}-\omega_{L O}$

$\mathrm{c} \varepsilon_{0}=\frac{1}{Z_{0}}$, where Zo is the impedance of free space $(377 \Omega)$

$\theta$ is the phase difference between $\mathrm{E}_{\mathrm{LO}}$ and $\mathrm{E}_{\mathrm{S}}$

$\gamma$ is the heterodyne mixing efficiency

Equations 2.4.1 and 2.4.2 are comprised of three parts: the incident power at the detector due to the local oscillator (LO); the incident power from the return signal (reflected from the target); and the interference power, given by the third term in Eq. 2.4.2. It is this term that carries the signal information (amplitude and phase). Simple inspection shows that $\mathrm{P}_{\mathrm{LO}}$ can be arbitrarily increased in power (up to the point of saturation of the detector). It is this feature that makes coherent detection ladar so much more sensitive than direct detection. The amplification process occurs in the optical domain (via mixing with an optical LO), before any thermal noise gets into the system. In direct detection usually the amplification is done in the electrical domain (i.e., electron gain), which amplifies noise as well. Coherent LADARs can be designed to be shot noise limited. The significance of this is discussed in Section 2.4.1. 


\subsubsection{Hardware Variations}

An unmodulated continuous-wave source is suitable for velocity measurements but is incapable of measuring range [Kamerman, 1996; Lange 2000]. If range information is required the transmitted beam must be modulated separately from the LO. This is typically accomplished, for fully coherent ladar, by generating a chirp waveform (Figure 2.4.2) in the frequency domain. The methods of achieving this are conceptually simple yet difficult to practically implement in a stable, linear system. One approach is to thermally control the laser cavity; as the cavity expands and contracts under thermal excitation the coherent wavelength of the laser changes. Similarly, one could change the length of the cavity mechanically using extremely fast piezoelectric actuators, or modelock the laser excitation to an ultra-stable oscillator whose frequency is changed using similar mechanisms. Achieving repeatable linearity over an ultrawide bandwidth (see

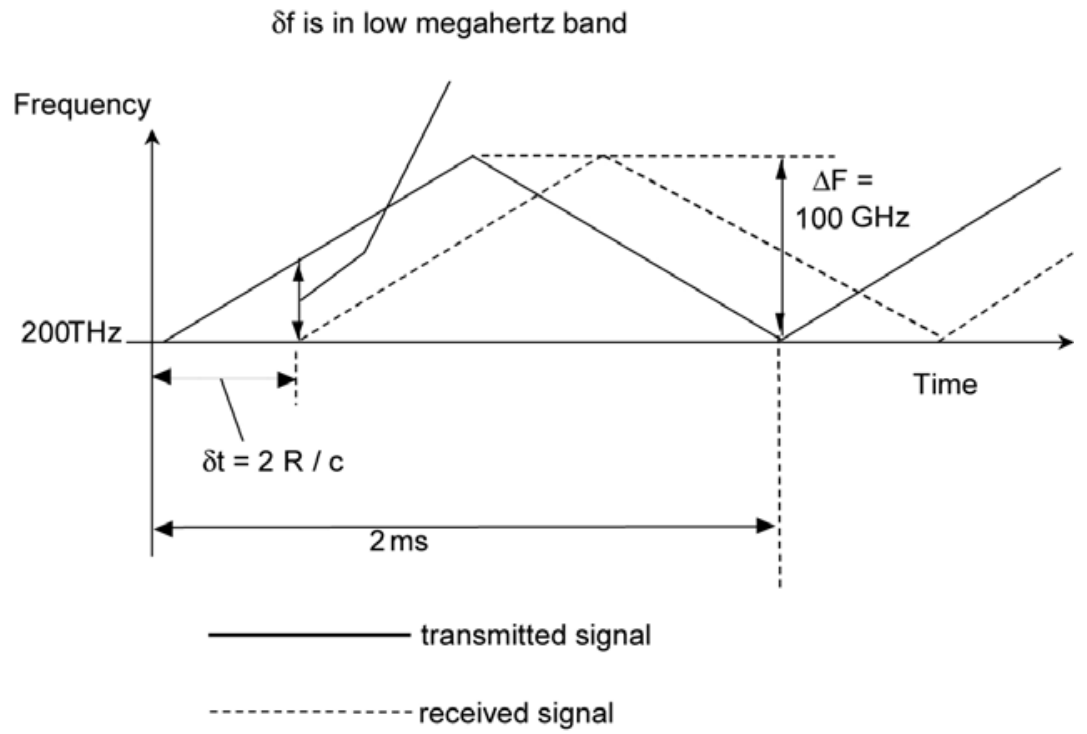

Figure 2.4.2: Frequency chirp (FM-CW) used in the Metric Vision MV-200 coherent laser radar. Compare this with the chirped AM architecture discussed in Section 2.3 [Courtesy Metric Vision]

Figure 2.4.2) is the crux of the design.

Using coherent detection principles it is feasible to obtain very high range resolution and accuracy within a specified range band (in general, the smaller the range band the higher the achievable accuracy, unlike pulse time of flight devices). This can be understood, again, in terms of Figure 2.4.2. Because the range information is contained within the mixed, intermediate frequency (IF) signal (the third part of Eqs. 2.4.1 and 2.4.2) for close targets there will only be a small IF frequency shift between the transmitted and received signal. A small frequency difference means close range; a long frequency difference ( $\delta f$ in Figure 2.4.2) means long range. In this situation, the detector bandwidth only has to accomodate the range of interest (e.g. $0 \mathrm{~m}$ to $20 \mathrm{~m}$; or $200 \mathrm{~m}$ to $400 \mathrm{~m}$ etc). The range resolution, following classical radar theory, is given by Eq. 2.3.1 (reprinted): 


$$
\Delta R=\frac{c}{2 \Delta F}
$$

This is defined by the Rayleigh Criteria as the range extent of a waveform for which two equal strength point targets produce a combined double peak echo which dips down to $1 / 2$ of the peak values between the two targets. However, with phase-based measurements, the precision can far exceed the resolution. As the carrier to noise ratio (CNR) increases, the measurement error decreases. In addition, if one averages a measurement 10,000 times, the precision of the result is 100 times better than if one did not average the measurements [Gatt, 2003]. Yet the resolution of the waveform is unaffected by the CNR or the number of averaging measurements $(\mathrm{N})$. It is an intrinsic property of the transmitted waveform, not the receiver characteristics (see Section 2.4.2).

As an example, the chirp shown in Figure 2.4.2 has a slope of $100 \mathrm{GHz}$ in one millisecond. This means the laser frequency is being "tuned" at $575 \mathrm{kHz}$ per meter. If the input signal is digitized and we then perform a 1024 bin FFT, where each bin is $254 \mathrm{~Hz}$ in width, the corresponding range resolution bin size is $0.442 \mathrm{~mm}$ wide. It is possible at this point to employ "super-resolution" interpolation algorithms [Stoker, 1998] -- e.g. a 3-point cubic fit using the largest bin and its neighbors -- to achieve sub-bin resolution. The accuracy (or repeatability) of this interpolation is dependent upon the original SNR. It also depends upon how many waveforms we stack or average prior to performing the FFT (which increases the SNR) [Slotwinski, 2003]

Further, if a particular resolution is desired that can be obtained in the range of, say, $0 \mathrm{~m}$ to $20 \mathrm{~m}$, one can extend the refined measurement window to any desired starting point by putting a delay between the local oscillator and the receiver. This could be achieved, physically, through the use of a $1 \mathrm{~km}$ spool of fiber optic line connected to the output end of the LO and to the input end of the optical mixer before the detector. The effective high resolution window of measurement would then be $1000 \mathrm{~m}$ to 1020 $\mathrm{m}$. Some high resolution coherent laser radar (CLR) devices make use of this technique to successively bound range zones to continuously cover larger ranges at high resolution (e.g. $0 \mathrm{~m}$ to $20 \mathrm{~m} ; 20 \mathrm{~m}$ to $40 \mathrm{~m}$ etc) within the context of a single instrument.

Thus far we have spoken only in terms of the simple schematic given in Figure 2.3.1. A more detailed system design example is given in Figure 2.3.3. Coherent laser radars were in fact among the first class of LADAR to be developed. As such, mixing optics were typically in the form of glass beam splitters ("BS" in Figure 2.3.3). These types of systems have very high construction precision requirements on the optical system. In order to generate the IF signal the signal and the local oscillator must be spatially and temporaly coherent, well aligned with respect to each other, and have identical polarizations. If these conditions are met then the two optical signals generate temporal interference at a frequency equal to the frequency difference between them [Kammerman, 1996]. 


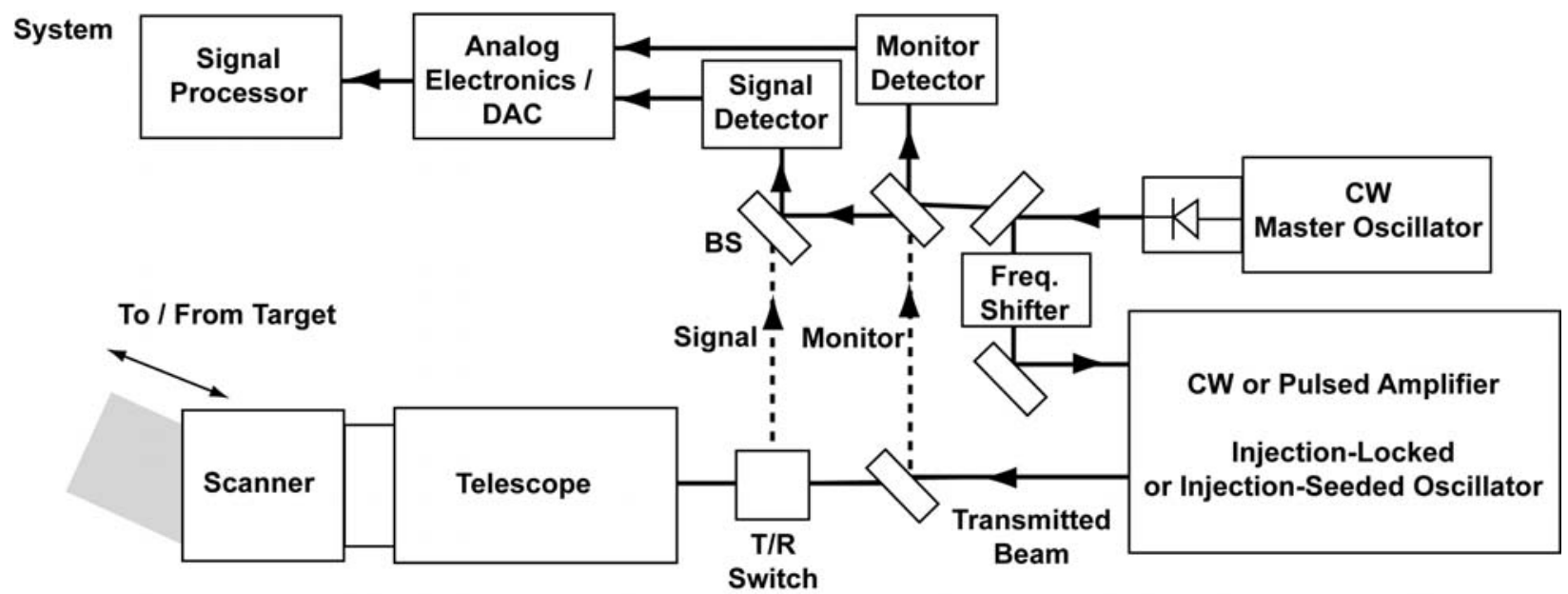

Figure 2.4.3: Frequency chirp (FM-CW) used in the Metric Vision MV-200 coherent laser radar. [Courtesy Metric Vision, Inc.]

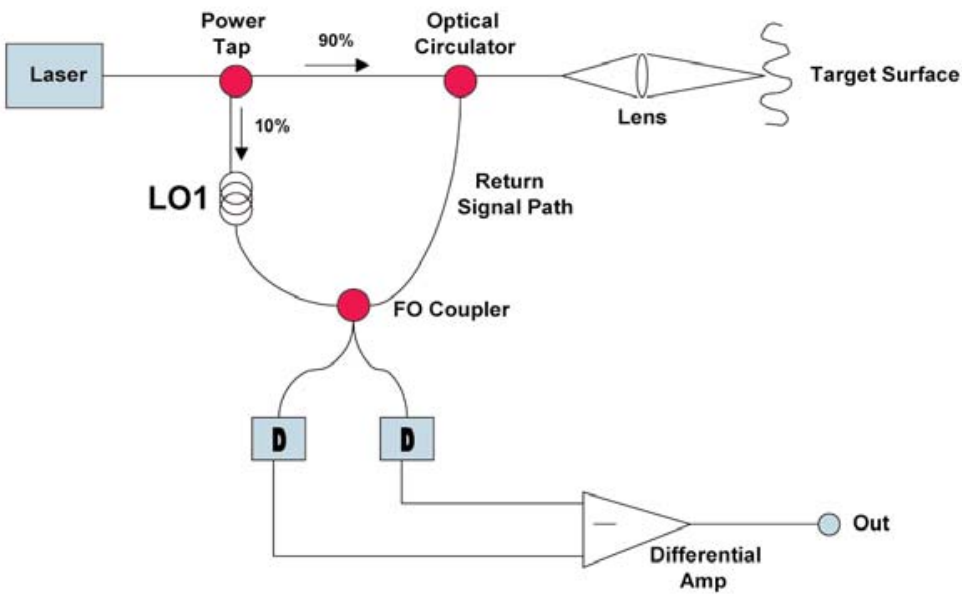

Figure 2.4.4: Modern CLR implementation employing telecom fiber optic routers and junctions. LO1 stands for Local Oscillator 1. The light in the LO1 path and in the signal path must come from the same source. If a different laser source were used in the LO1 path, then the smallest drift or variation in one of the lasers' frequency would cause large range errors. The only way to avoid this would be to mode lock one laser to the other which would be expensive and difficult. FO stands for Fiber Optic. D stands for detector. Dual detectors can be used for two purposes. First, to collect as much signal power as possible. Fiber optic $2 \times 2$ couplers split the input light into the two outputs so that the light collected from both outputs can be used to maximize the signal. In addition, the mixing process in the coupler is such that the signal in the two outputs are 180 degrees out of phase with each other while the laser intensity noise is in phase. By using the differential amplifier, the intensity noise can be cancelled (common mode rejection) while doubling the signal. For most CLR designs intensity noise is not an issue and most such designs employ fiber optic $1 \times 2$ couplers and a single detector. [Courtesy Metric Vision] 


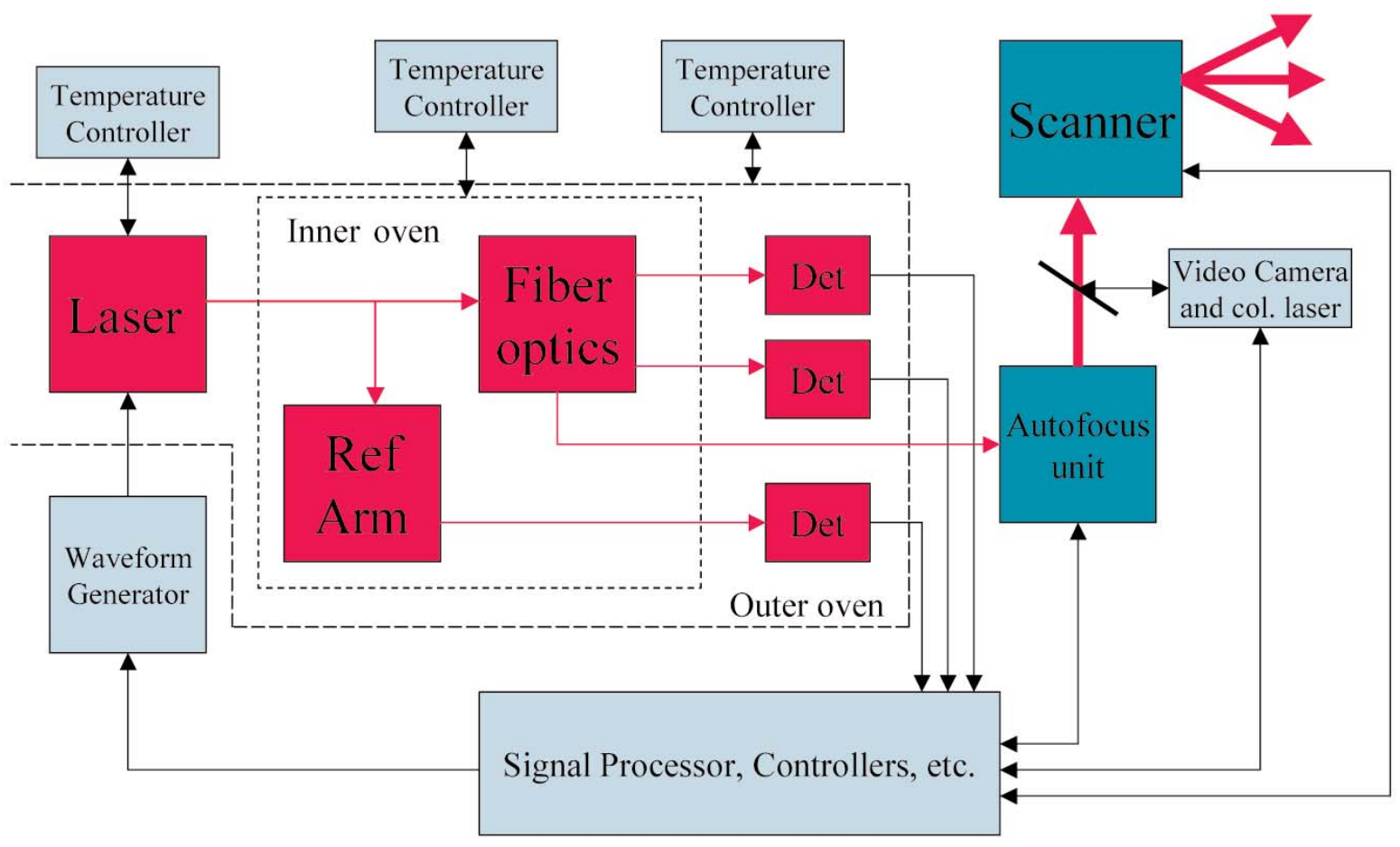

Figure 2.4.5: Commercial FM-CW CLR architecture using fiber optic connections. For improved accuracy of this metrology-level instrument (2 ppm accuracy) all fiber optic connections and delay lines are maintained in a temperature-controlled oven. The unit also includes a beam auto-focus for improving the signal-to-noise ratio (SNR). See Section 2.4.2 for a discussion of the accuracy of CLR sensors. [Courtesy Metric Vision]

\subsubsection{Range Accuracy for Coherent Laser Radar}

Unlike AM-CW LADAR ranging, the accuracy for a coherent laser radar (CLR) is ultimately controlled not only by the chirp bandwidth, but also by the signal to noise ratio at the detector and the number of samples $(\mathrm{N})$ acquired per measurement. One of the most succinct summaries on this subject is given by Slotwinski [Slotwinski, 2003]:

"The signal generated by a Coherent Laser Radar (CLR) system is proportional to the amount of light reflected off the target and recaptured by the focusing lens. Thus, the signal depends greatly on the target's surface roughness, reflectivity and absorption properties. For an opaque solid composed of a number of individual scatterers that have dimensions on the order of the wavelength of light, the scattered field is Lambertian where the reflected light is confined to a solid angle of $2 \pi$ steradians oriented normal to the surface. Therefore, the amount of optical power captured by the lens is proportional to the area of the lens' aperture and inversely proportional to the 
surface area of the hemisphere of scattered light at the target range, as shown in Equation (2.4.4):

$$
P_{R}=\frac{P_{T} \frac{\pi d^{2}}{4} \rho}{2 \pi R^{2}}=\frac{P_{T} \rho d^{2}}{8 R^{2}}
$$

where:

$P_{R}=$ received power

$P_{T}=$ transmitted power

$\mathrm{d}=$ lens aperture diameter

$\rho=$ target reflectivity

$\mathrm{R}=$ target range

Eq. 2.4.4 incorporates the target reflectivity, $\rho$, and the space loss. A third term, transmission loss $\left(L_{T}\right)$, must also be included. In general, transmission loss is defined as the fractional amount of optical energy that remains after the light is attenuated by such factors as lens component reflections, system losses, and atmospheric attenuation. Due to relatively short range application of this sensor, atmospheric effects can be ignored and the losses associated with the antenna lens and the system dominate. If $P_{T}$ is the power transmitted by the laser source, the signal power at the detector chip, $P_{S}=P_{R} L_{T}$ is then given by:

$$
P_{S}=\frac{P_{t} \rho L_{T} d^{2}}{8 R^{2}}
$$

where $L_{T}$ is the two way fractional transmission loss for the lens and the system.

In any optical system, the minimum detectable, or quantum limited, power, $P_{Q}$, is given by:

$$
P_{Q}=\frac{h v B}{\eta}
$$


where:

$\mathrm{h}=$ Plank's Constant

$\mathrm{v}=$ Optical frequency

$\mathrm{B}=$ Measurement bandwidth

$\eta=$ Detector quantum efficiency

The condition for quantum limited operation of a heterodyne receiver is that the shot noise produced by the local oscillator (L.O.) in the detector is sufficient to override the thermal noise in the amplifier. If the current generated by the L.O. is much greater than both the photodetector dark current and the signal current, then the heterodyne noise equivalent power (NEP) is given by:

$$
(N E P)_{\mathrm{Het}}=\left[\frac{h v}{\eta}+\frac{2 k T}{P_{L O}}\left(\frac{h v}{\eta e}\right)^{2} \frac{1}{R}\right]
$$

where:

$\mathrm{k}=$ Boltzman Constant

$\mathrm{T}=$ Effective amplifier temperature

$\mathrm{R}=$ Receiver load resistance

$\mathrm{e}=$ Electron charge

The NEP is defined as the amount of signal power spectral density necessary to make the signal-to-noise in an optical receiver equal to unity.

For sufficiently large local oscillator power, $P_{L O}$, equation (2.4.4) reduces to the quantum, or shot noise limited value of $\left(P_{Q} / \mathrm{B}\right)$. For a wavelength of $1550 \mathrm{~nm}$ and a detector quantum efficiency of 0.6 , the shot noise limited NEP is $2.14 \times 10^{-19} \mathrm{~W} / \mathrm{Hz}$

In the case of the CLR, shot noise limited performance represents the most sensitive mode of operation. Thus, it is important to utilize an optical receiver that minimizes the thermal noise and to ensure that the LO power is sufficient to operate in this mode.

From equation (2.4.2) and (2.4.4), and including a term for excess laser noise (NEP), the CLR signal-to-noise ratio (SNR) can be represented as:

$$
S N R=\frac{P_{t} \rho L_{T} d^{2}}{8(N E P)_{H E T} R^{2} B N_{e}}
$$


where $N_{e}$ is the laser excess noise factor. This term results from the fact that lasers are not ideal "noiseless" sources, but have noise in excess of the quantum noise that is frequency dependent. For the diode lasers used in such systems, typical values for $N_{e}$ at frequencies greater than $1 \mathrm{MHz}$ range from 2 to 4 .

Equation (2.4.8) represents the case when the target is at the beam focus. If the target is at a position other than focus, defocusing loss occurs. This loss is given by: (for a Gaussian beam):

$$
L_{d}=\log ^{-1}\left(\frac{0.3|R-X|}{2.2\left(\frac{R}{d}\right)^{2} \lambda}\right)
$$

where:

$|R-X|=$ absolute value of the distance from the focus.

$X=$ focus position

The SNR is also affected by the coherence length of the source. For a Lorentzian shaped source linewidth, the fringe is given by:

$$
V=e^{-\left(\frac{2 \pi R \Delta f}{c}\right)}
$$

where:

$\Delta f=$ source linewidth

$\mathrm{c}=$ speed of light

As the measurement length increases, the loss due to coherence length increases as the square of the visibility. Thus, including the losses due to the defocusing and coherence, the SNR equation becomes: 


$$
S N R=\frac{P_{t} \rho L_{T} d^{2} V^{2}}{8(N E P)_{H e t} B N_{e} R^{2} L_{d}}
$$

The one sigma accuracy of a chirp radar range measurement is determined by the accuracy of measuring the frequency of a pure sinusoidal signal over a measurement interval (chirp period) T. This is given by:

$$
\sigma_{f}=\left(\frac{3}{2}\right)^{\frac{1}{2}} \frac{1}{\pi T^{\frac{3}{2}}\left(S N R_{o}\right)^{\frac{1}{2}}}
$$

where $=\mathrm{SNR}_{0}$ in a $1 \mathrm{~Hz}$ bandwidth (i.e. $\mathrm{B}=1 \mathrm{~Hz}$ )

The one sigma range accuracy, is thus determined by:

$$
\sigma_{R}=\frac{\sqrt{2}}{2}\left(\frac{c T}{\Delta f}\right) \sigma_{F}=\frac{\sqrt{3}}{2} \frac{c}{\pi(\Delta f) T^{\frac{1}{2}}\left(S N R_{o}\right)^{\frac{1}{2}}}
$$

where

$\Delta f=$ laser frequency excursion

$\mathrm{T}=$ sweep period $(1 /$ measurement rate)

As an example of of a practically-implementable system Table 2.4.1 provides minimum performance values for the various parameters in the above equations for the Metric Vision MV200 CLR. The defocusing loss is set to 1 denoting that the system is focused on the target. A target range of 10 meters was selected for this example.

From Equations (2.4.11) and (2.4.13), one can see that the theoretical range accuracy is a function of the target reflectivity. Table 2.4 .2 shows the single measurement accuracy for a number of different target types and reflectivities based on the parameters from Table 2.4.1. Note that with all phase-based systems it is possible to improve these numbers still further through longer integration times (that is, multiple sampling of the same target). 
Table 2.4.1 Typical parameters for the Metric Vision MV-200 CLR

\begin{tabular}{|l|l|l|}
\hline Parameter & Symbol & Value \\
\hline Transmitted Power & $\mathrm{P}_{\mathrm{t}}$ & $2 \mathrm{~mW}$ \\
\hline Transmission Loss & $\mathrm{L}_{\mathrm{t}}$ & 0.2 \\
\hline Lens Aperture & $\mathrm{d}$ & $40 \mathrm{~mm}$ \\
\hline Fringe Visibility & $\mathrm{V}$ & 0.5 \\
\hline Heterodyne Noise Equivalent Power & $(\mathrm{NEP})_{\mathrm{Het}}$ & $2.14 \times 10^{-19} \mathrm{~W} / \mathrm{Hz}$ \\
\hline Laser Excess Noise & $\mathrm{N}_{\mathrm{e}}$ & 4 \\
\hline Range & $\mathrm{R}$ & $10 \mathrm{~m}$ \\
\hline Defocusing Loss & $\mathrm{L}_{\mathrm{d}}$ & 1 \\
\hline Speed of Light & $\mathrm{C}$ & $3 \times 10^{8} \mathrm{~m} / \mathrm{s}$ \\
\hline Laser Frequency Excursion & $\Delta \mathrm{f}$ & $100 \mathrm{GHz}$ \\
\hline Sweep Period & $\mathrm{T}$ & $1 \mathrm{~ms}$ \\
\hline
\end{tabular}

Table 2.4.2 Theoretical Range Accuracies for Different Targets for the sensor as described in Table 2.4.1 [courtesy Metric Vision]

\begin{tabular}{|l|c|c|}
\hline Target Type & Typical Reflectivity $(\rho)$ & Range Accuracy \\
\hline Machined aluminum (normal incidence) & $1000 \%$ & $0.54 \mu \mathrm{m}$ \\
\hline White paper & $100 \%$ & $1.7 \mu \mathrm{m}$ \\
\hline Dark paper & $10 \%$ & $5.4 \mu \mathrm{m}$ \\
\hline Flat black paint & $1 \%$ & $17 \mu \mathrm{m}$ \\
\hline Black absorber & $0.1 \%$ & $54 \mu \mathrm{m}$ \\
\hline
\end{tabular}

As can be seen from Table 2.4.2, for all but the dimmest of targets, the theoretical range accuracy is less than $2.5 \mathrm{ppm}$ at a range of $10 \mathrm{~m}$. For targets brighter than about $1000 \%$ the SNR becomes limited by the coherence noise floor so the range accuracy does not increase beyond this point. Also, accuracy can be increased by averaging successive single sweep measurements with the accuracy increasing with the square root of the number of averages (that is, $\sigma_{R}$ in Eq. 2.4.13 will decrease inversely with the square root of the number of measurements).

In the real world, absolute accuracies below a few parts per million are difficult to achieve due changes in the air's temperature, pressure and humidity which affect the laser beam's time of propagation (See Chapter 4). Metrology-level LADARs frequently contain a "weather station" to compensate for these parameters but gradients along the measurement path will degrade the accuracy. Also, during assembly, a standard interferometer is frequently used to calibrate each sub-system. The absolute accuracy of the calibrating interferometer will limit the absolute accuracy of the metrology sensor. 
Target surface effects also limit the accuracy of CLR sensors. Surface range noise due to speckle and beam penetration can cause increases in the range error. This can be significant for some types of targets. In general, the best system performance is achieved off tooling ball targets [Slotwinski, 2003].

The above discussion has been limited to a "0-D" (that is, single point) CLR ranging system. As with similar pulse time-of-flight 0-D systems, such a CLR would have to be scanned in order to create a range image. The requirement for optical mixing places severe constraints on the implementation of a CLR focal plane array. However, for situations where extraordinary resolution within a specified range gate and/or direct velocity measurements are considered crucial, then CLR is a logical solution. 


\subsection{Beam Scanning Technology}

The following observations are a result of a contemporary assessment of this technology as well as ongoing research at NIST, both in laser deflection for optical space communications and, more directly, associated with extremely high speed deflection systems for LADAR.

With the exception of pure flash (FPA) systems (and even some of these are steered) most LADAR systems must sequentially illuminate each pixel in a range image. The methods for achieving this are referred to variously as scanning and beam-steering. The Next-Generation LADAR issue with beam steering is simply that existing methods are too large, complex, and bulky for low cost, compact, mass-production implementation. The mirrors, prisms, and spinning polygons used in current technology are all made of precision glass or ceramics in forms controlled by macro fabrication technology. Their high mass and geometry in turn impose physical (stress) limits to achievable angular deflection rates. Microfabrication may point the way onward. With reduced size comes reduced inertia, which in turn permits higher performance. Likewise, with substantially reduced size new avenues for fabrication - avenues inherently predisposed to mass fabrication - become available.

In this section we rapidly review the technology of commercially available beam steering mechanisms and then proceed to describe a new concept of beam array micro scanners. These are small size devices with overlapping workspaces, which would allow higher resolution mapping (zooming) of any desired location in the workspace, with a vastly higher angular deflection rate being a predictable bonus.

For an excellent, in-depth discussion of many of the existing devices described below through 1996 the reader is referred as well to Montagu and DeWeerd [1996]. We list these current technologies here briefly for completeness before providing more detailed discussion of the approaches currently being investigated at NIST.

\subsection{Single Beam Scanners}

The largest application of single beam scanners is for reading and writing information of supermarket products, copier documents and optical disks, [Marshall, 1991], [Gottlieb, 1983]. High resolution and high scanning speed are the main performance requirements of these scanners.

\subsubsection{Polygonal Scanners}

High speed uniform scanning are the main advantages of polygonal scanners. The most common errors of this type of scanners are bowtie and line cross-scan. If the plane of the incoming and reflected beams is not perpendicular to the polygon axis of rotation a curved scan line results. If two polygonal scanners are used, in order to generate a 2D scan, a characteristic bowtie shape is generated. Wobble of the poly- 
gon rotation and pyramidal errors of the reflecting surfaces result in cross-scan errors of the scan lines.
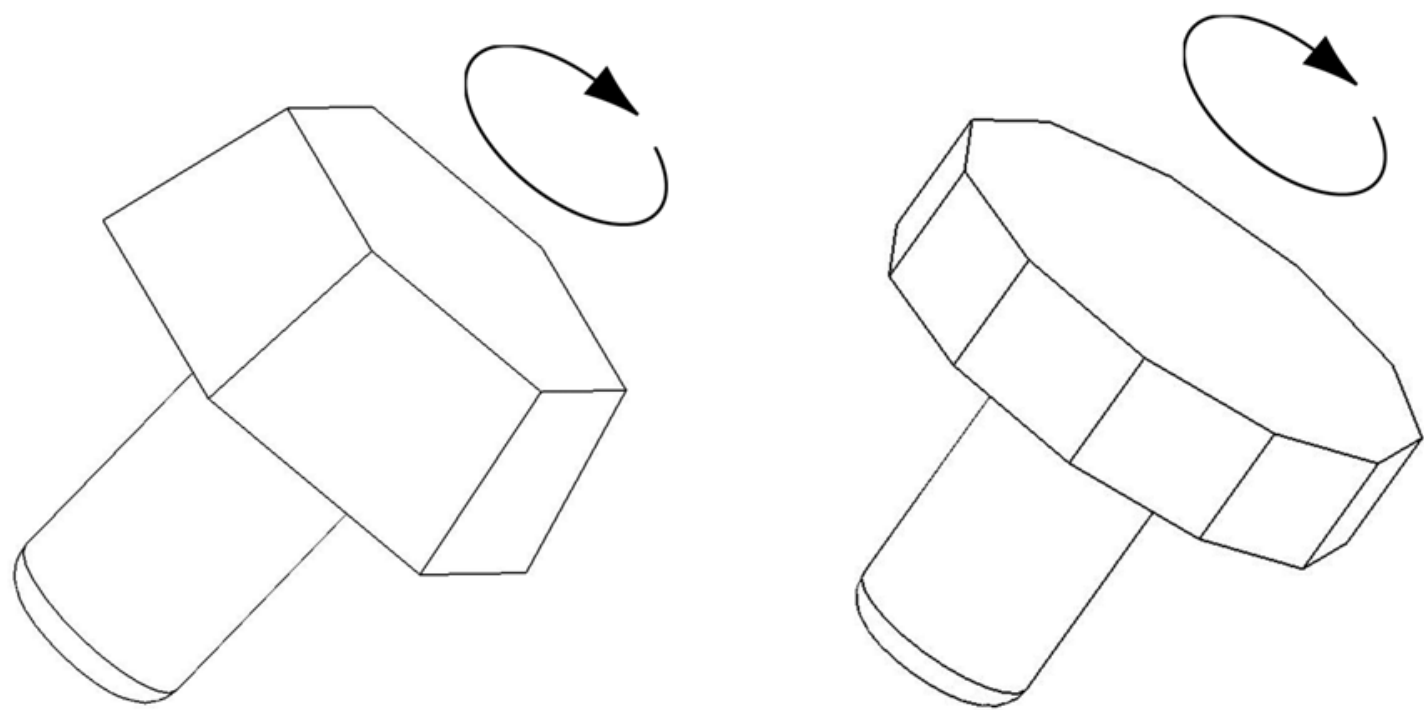

Figure 3.1.1. Typical examples of polygon mirror deflectors. Rotational speeds can reach several thousand revolutions per second.

\subsubsection{Galvanometric Scanners}

Galvanometric scanners are very popular. Like in the case of polygonal scanners the two reflecting surfaces, necessary in order to create a 2-D scan, are separate from each other thus complicating the optics design. Compared to polygonal scanners these scanners have the great advantage that the axis of rotation can lay on the plane of the reflecting surface. These scanners can have bowtie and line cross-scan errors for reasons given in the polygonal scanners section.

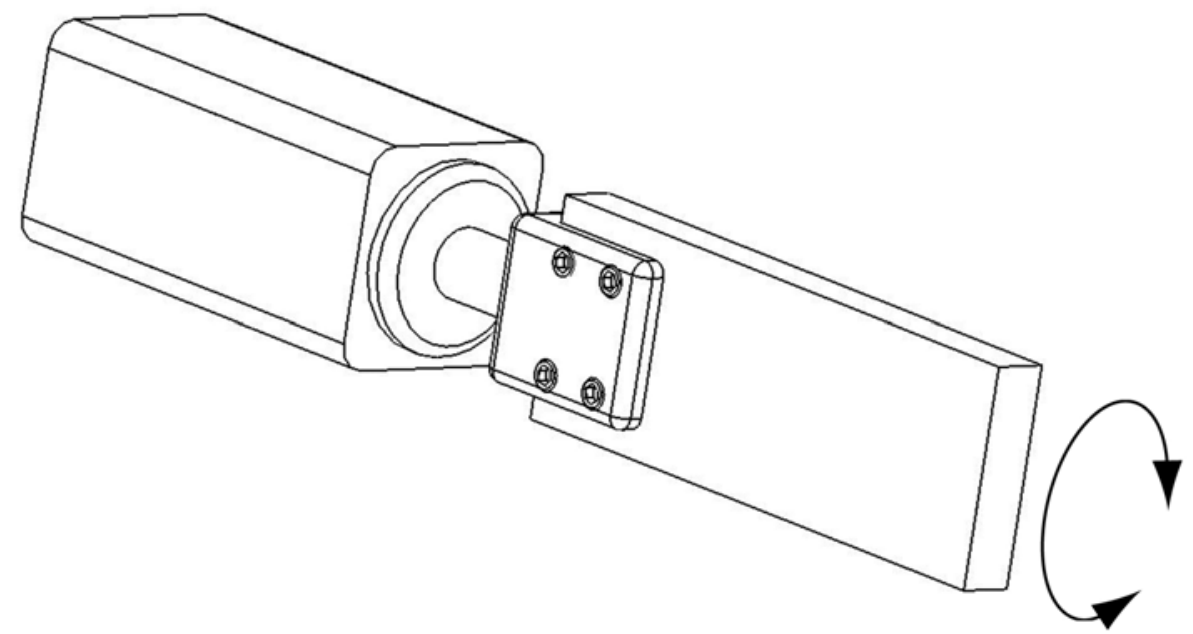

Figure 3.1.2. Typical example of a galvonometer-driven planar mirror. These are typically referred to as "galvos" in the industry. 


\subsubsection{Acousto Optic Scanners}

Acousto-optic devices use acoustic waves propagating in a variety of optic materials to control the refractive index of the material and thus the angle of the output light beam. A typical 2-D scanner uses a pair of acousto-optic beam deflectors and has an angular deflection range of up to 10 degrees and sweep frequency of a few $\mathrm{KHz}$. An acoustic wave passing through an optic device behaves like a sinusoidal grating that diffracts the laser beam. The angular deflection of the most efficient power first order beam is proportional to the frequency of the acoustic wave and is used for scanning. One problem with this type of scanners is the large absorption of the laser beam, typically $80 \%$ to $85 \%$ depending on wavelength.

\subsubsection{Electro Optic Scanners}

Electro-optic scanners operate by changing optical material properties through the application of voltages to photoelastic materials. The varying input voltage can be used to change the polarization of the illumination beam which in turn affects the degree to which the beam can be deflected through the appropriate material. The deflection angle is dependent on the wavelength of light, with longer wavelengths allowing greater deflection. Response times can be as low as a microsecond but total deflection is limited to less than 2 degrees.

\subsubsection{Holographic Scanners}

Holography is an optical information storage process where the amplitude and phase information of the light illuminating the recording medium create permanent changes to that medium, which can be used later in order to generate desired waveforms under proper illumination. A hologram can be designed to act as a conventional refracting lens. Shining a small diameter beam onto such a hologram results in a deflection and focusing of that beam. A relative translation of the hologram or the beam about a plane parallel to that of the hologram results in a scanning angular motion of the output beam [Beiser, 1988]. Such a motion can be generated by an XY translation micro positioner, which may carry the hologram or an optical fiber. Laser beam scanners of that type will be described in the micro positioners beam arrays section. A more common mechanism is to etch holographic patterns on the periphery of a disk and then spin it in front of the illuminating beam. This is the predominant scanning mechanism used by super market check out counter machines. A hologram can be designed to generate variable aperture, variable focal length beams and variable scan angle gain. As in the case of polygonal scanners, holographic scanners may have bowtie, wobble and pyramidal errors.

\subsubsection{Tilt Platform Scanners}

These are one, two or three axes compact fast steering mirror devices that are used for a variety of diverse applications. They have cylindrical or rectangular shapes with 
typical dimensions of a few $\mathrm{cm}$ length by a few $\mathrm{cm}$ diameter. They can tilt a reflecting mirror about one or two orthogonal $\mathrm{XY}$ axes and some of them can translate the mirror about a third orthogonal $Z$ axis. They are actuated by piezoelectric or voice coil actuators, can tilt very fast and depending on the size of the mirror can have a bandwidth of several hundred $\mathrm{Hz}$. The tilt platform structure is usually built from a single piece of annealed metal with a deforming structure suspension, which eliminates backlash and stiction errors, very common with conventional motion systems. High quality tilt platforms are equipped with capacitance or strain gage displacement sensors, which measure the angular displacement of the mirror and can be used for closed loop control thus generating high accuracy and repeatability scans. Two possible sources of error are cross talk and thermal drift. With the use of high quality materials, good design, machining and the use of pairs of actuators [Physik Instruments 1] it is possible to minimize these errors. Typical range that can be achieved by tilt platform devices varies from a fraction to a few degrees of angular displacement.

\subsection{Beam Array Scanners}

The Defense Advanced Research Programs Agency (DARPA) is funding a program on Steered Agile Beams [STAB, 2000]. According to its program overview this program "is developing small, lightweight laser beam scanning technologies for the replacement of large, heavy gimbaled mirror systems." This program is funding several projects, which are developing "solid state/micro-component technologies such as optical MEMs, patterned liquid crystals, diffractive micro-optics and photonic crystals (that) will be used to build small, ultra-light, rapidly steered laser beam sub-systems." The target applications of these technologies are free space optical communications and electrooptics countermeasures.

In [Dagalakis et al., 2002] we proposed the use of laser beam arrays for nano assembly. Here we propose to expand the application of this technology to LADAR sensors. It is expected that this technology can reduce the size and cost of the LADAR scanners and can increase resolution. The environment mapping application of LADAR dictates the use of very accurate and precise micro scanners. The micro positioner beam scanners that we are developing have the potential to meet that requirement.

\subsubsection{Scanning Micro Mirrors Beam Arrays}

For more than a decade the research laboratories of many semiconductor manufacturers and telecommunications equipment manufacturers have been working hard on the development of micro-mirror arrays (MMA). The first applications were in digital projection equipment, which has now expanded into digital cinema projectors, with sometimes more than two million micro-mirrors per chip switching at frequencies of up to 66 $\mathrm{kHz}$ [Younse, 1995; Texas Instruments, 2004; DLP, 2004]. Later these MMAs were used on telescopes to enhance fuzzy images and studies are underway to use them for the next generation space telescope and multi space object spectrometer [Sandia, 


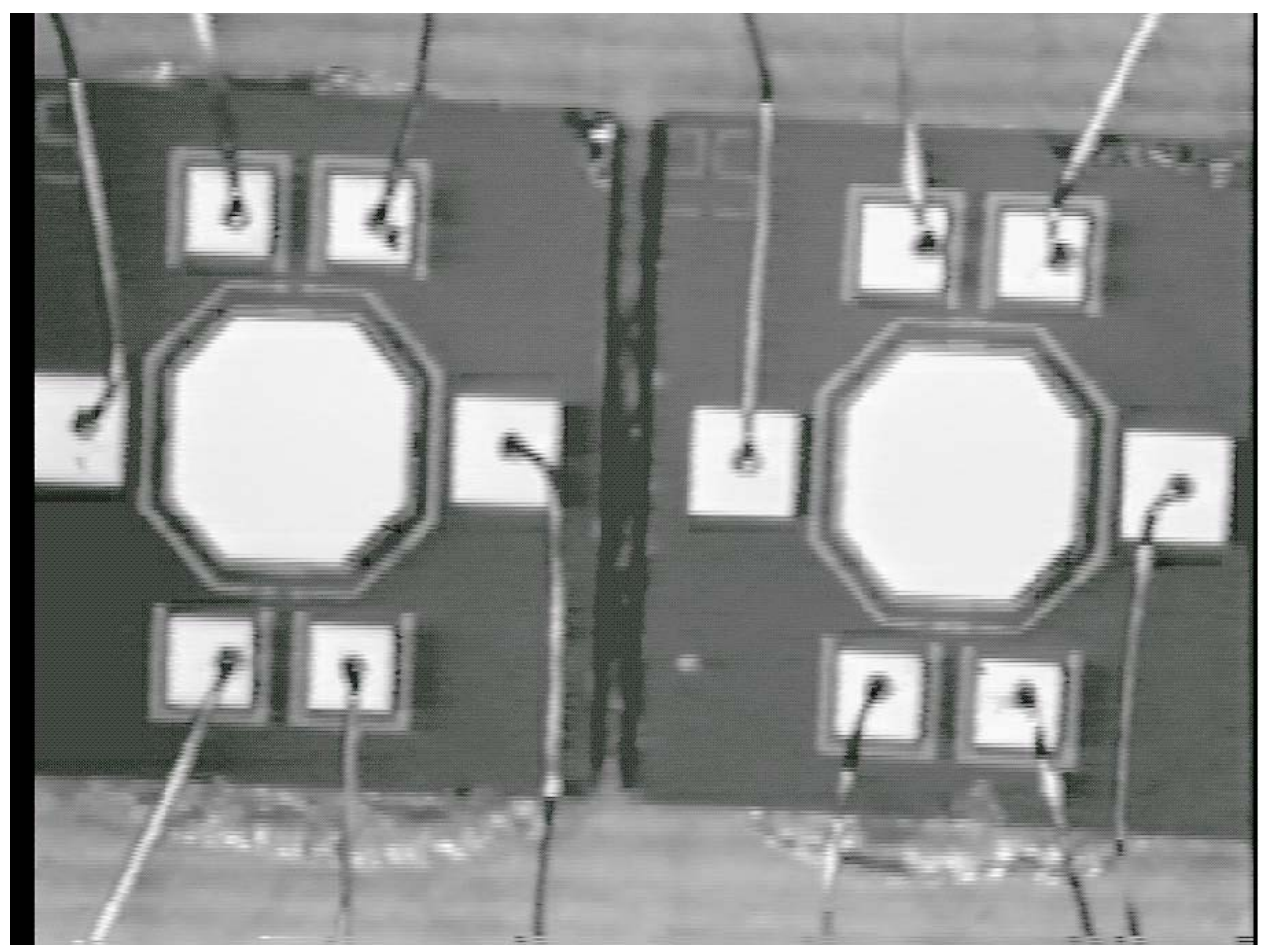

Figure 3.2.1. Two of the array micro-mirrors used in the NIST beam deflection experiments

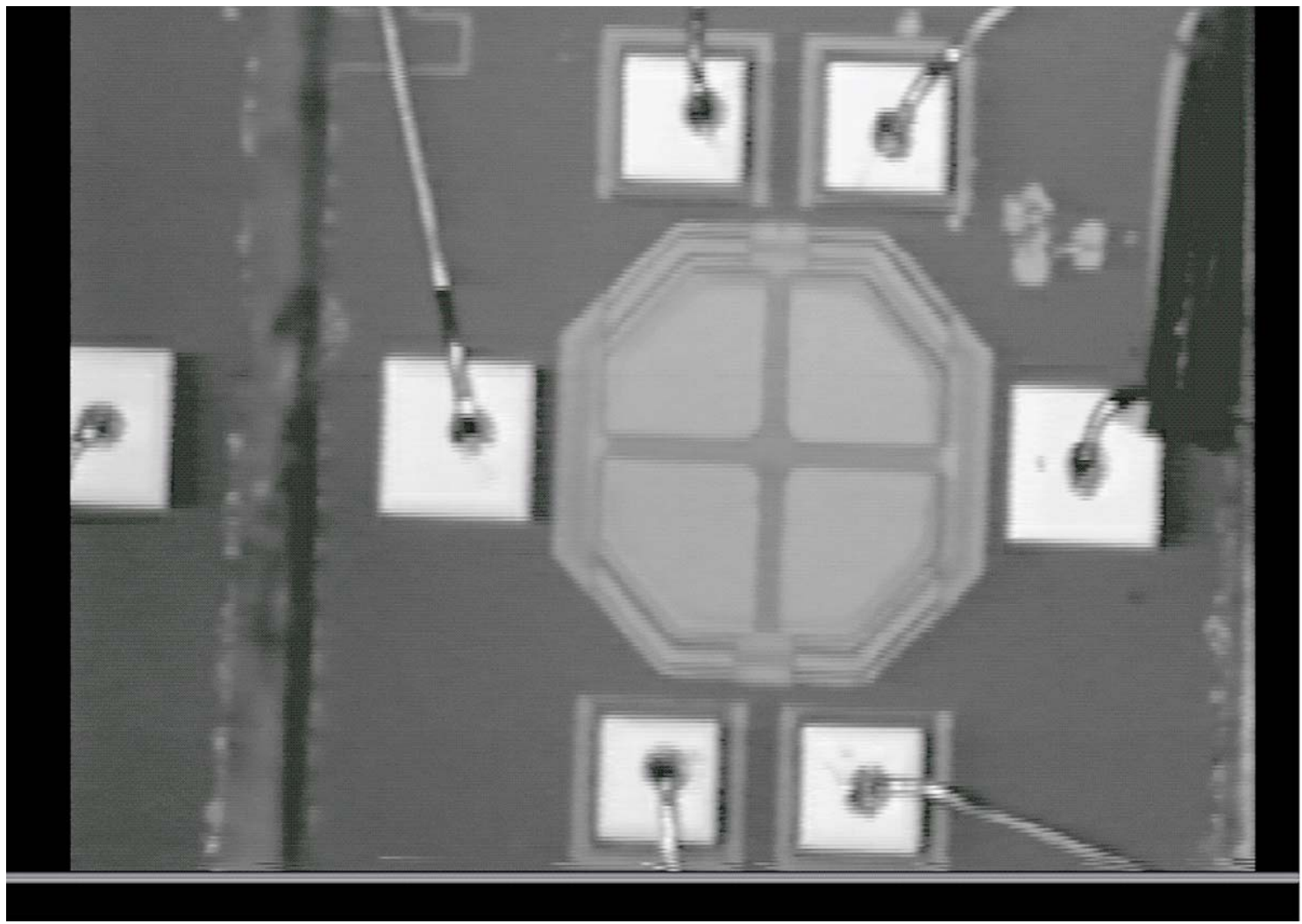

Figure 3.2.2. Micro-mirror electrostatic actuation pads 
1999]. Recently MMAs are finding applications in the large telecommunications market as optical multiplexers and cross-connect switches. State of the art devices have 512 switches, while it is expected that this number could rise to 10,000 in a few years [Lucent, 2004]. Most MMAs are fabricated from silicon and thus fall into the general category of Micro-Electro-Mechanical-Systems (MEMS). The size of an MMA mirror is usually less than a mm across, requires a very small amount of power to move it, and can move fast - typically in milliseconds.

The first generation of MMAs was binary, which means that the micro-mirrors could only assume two positions. In the last few years a new generation of MMAs is being introduced, which are equipped with servo control. These are sometimes referred to as Scanning MMAs (SMMA), because the micro-mirror tilt angle is a function of the input command signal. We have started experimenting with a Scanning Two Axis Tilt Mirror MEMS Optical SMMA [MEMS Optical, 2004]. These SMMAs are available in arrays of 4 by 1 and 8 by 1 micro-mirrors. We are currently using a 4 by 1 SMMA. The size of these micro-mirrors is $520 \mu \mathrm{m}$ across and they have an octagonal shape. Fig. 3.2.1 is a magnification of two of those micro-mirrors. The micro-mirrors are actuated by electrostatic actuators (Fig. 3.2.2), which are located behind the reflecting front face of the mirrors. They consist of four capacitor pads separated by two orthogonal channels parallel to the two axes of rotation of the corresponding micro-mirror. The mirror is grounded and the four pads are placed under a bias voltage to mechanically preload the mirror. By modulating the voltage of the four pads about the bias level it is possible to generate controlled rotations of the micro-mirrors. The range of rotation is $+/-3^{0}$, which corresponds to $a+/-6^{0}$ of laser beam rotation. The manufacturer-measured resonant frequencies are approximately $1.4 \mathrm{kHz}$ for one axis and $1 \mathrm{kHz}$ for the other. Another company [Applied MEMS, 2004] has a similar product with a rotation angle of $+/-14^{\circ}$, which corresponds to $a+/-28^{\circ}$ of laser beam rotation and capacitive position sensing of the mirror plate. Unfortunately their micro-mirror array is not yet commercially available.

Micro-mirror arrays could prove to be a very useful technology for the control of the resolution of LADAR sensors. Micro-mirrors can act as a distributed scanner that generates a large number of micro-beams that can scan the workspace from different angles and positions. An alternative use of the micro-mirror arrays could be that of an optical switch that routes a laser beam to various optical fibers, which then become the generators of the Ladar beam scanning action. In order to improve and control the resolution of LADAR sensors we propose the use of arrays of micro-beam scanners, which we feel will offer an advantage as compared to single motorized scanners or fixed position emitter-receivers. We are testing various techniques to split laser beams with arrays of micro-scanners and to generate micro-beams that can scan in many directions from different locations. In terrain locations where sharp transitions are detected, directed scanning from multiple micro-scanners of overlapping workspaces could be used to increase the mapping resolution (directed multi-mirror scanning). This is equivalent to several people observing the same scene from different locations 


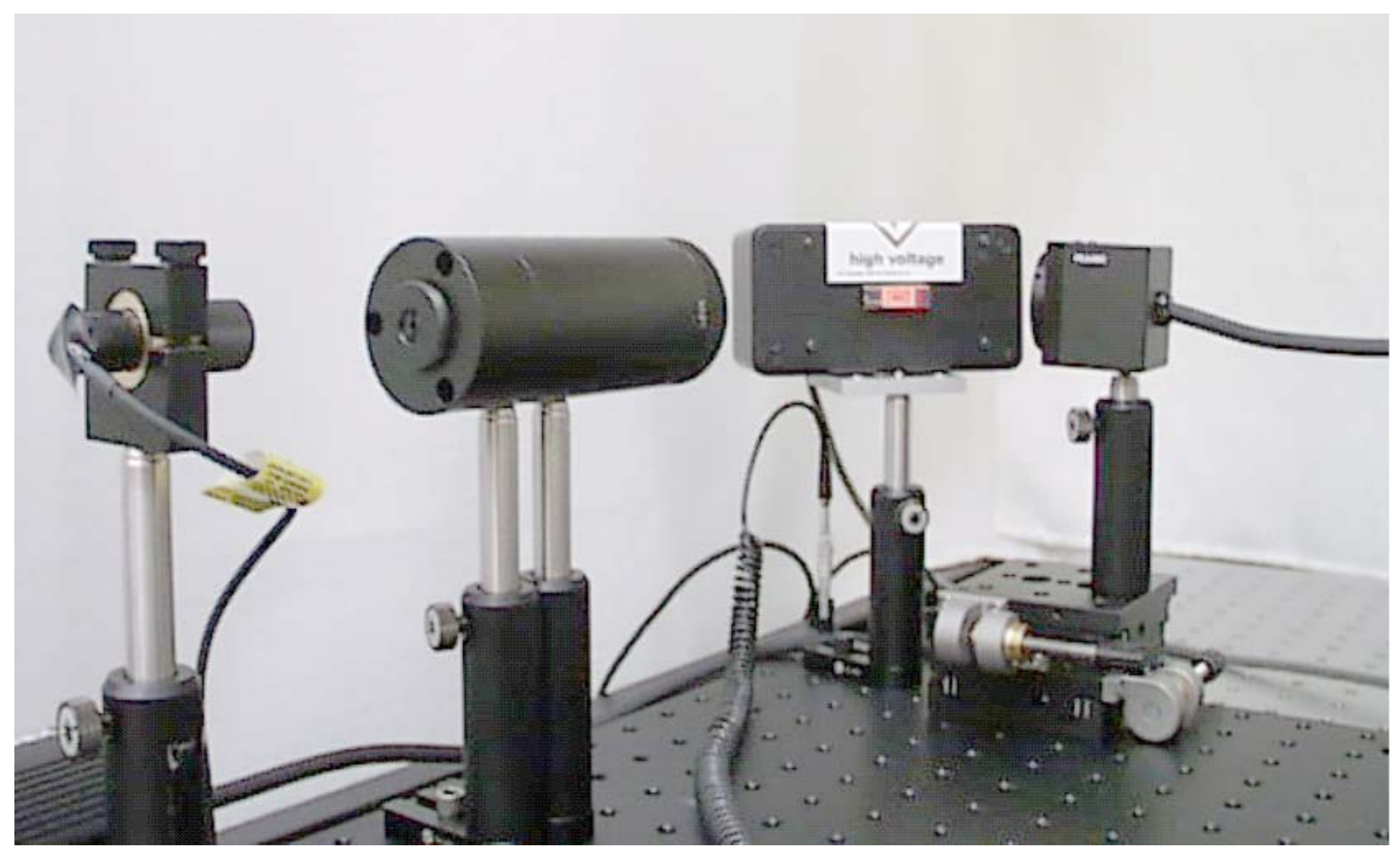

Figure 3.2.3. Micro-mirror with electrostatic actuation pads

and directions. The terrain observations are collected by the same controller and are combined to provide a high-resolution 3D image of the scene. The calibration and control of these distributed sensors is a very challenging problem. Stitching together (i.e. registering) and properly displaying multiple images generated by arrays of microscanners is going to be a difficult problem.

Fig. 3.2.3 shows a picture of a micro-mirror array chip that is being calibrated in our lab. The chip is mounted on a socket, in the middle of the picture and is illuminated by a laser beam. There are several ways to illuminate the mirror arrays in order to generate an array of laser micro-beams. The simplest technique is flood illumination, preferably with a beam that is shaped to have a cross-section similar to that of the mirror array [Dagalakis et. al., 2002]. The main advantage of this technique is simplicity, while the main disadvantages are that a portion of the beam power is not utilized and that the micro-mirror surrounding structures might generate unwanted reflections. Another illumination technique would be to aim optical fibers to the center of each micro-mirror. This technique would require the careful calibration of the optical fiber array and the use of an optical switch to route the laser power into each optical fiber with the desired timing. 


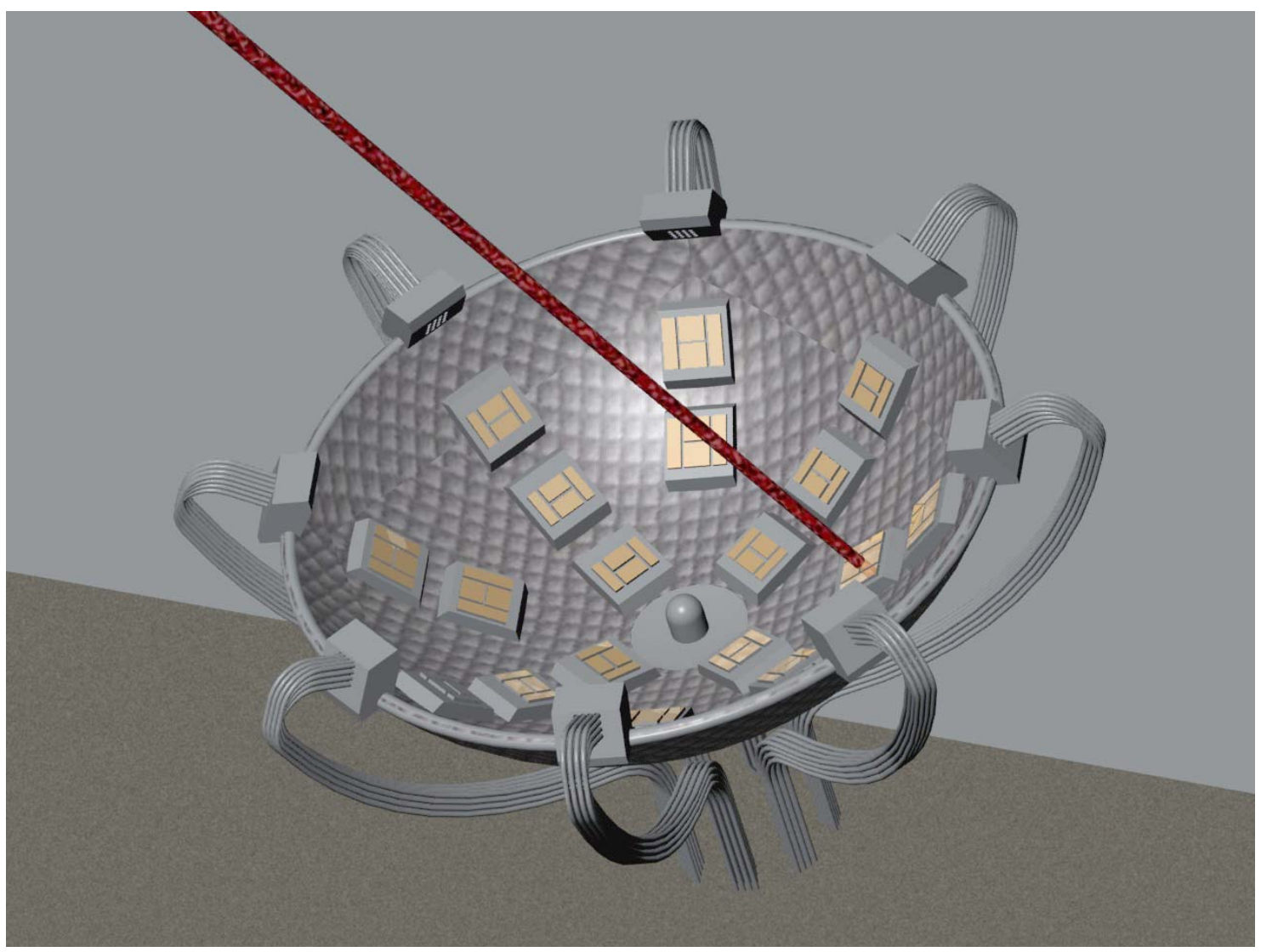

Figure 3.2.4. Micro-mirror array scanner concept under development at NIST

Since the mirror arrays are built on chips that are mounted on sockets they can be located anywhere in the workspace. Furthermore, a socket-supporting plate could be built that is optimized for the resolution requirements of a particular workspace. Fig. 3.6 shows an example of such a plate with a concave surface. Mirror array chips are mounted at different locations on this surface, while the reflected beam sensor is located in the middle. The laser beams are generated by laser diodes or optical fibers located on the periphery of the mounting surface. One beam and one micro mirror are used in order to demonstrate the scanning ability of these devices. It is obvious that the size, shape and curvature of the mounting surface determine the available size of the scanned workspace. If the operation of the micro-mirrors is coordinated by a central controller it is possible to achieve flexible scanning and to zoom to certain segments of the workspace where higher resolution is necessary. The SMMA response to the input command signals is non-linear and requires a good controller for high performance operation. Micro-mirrors with feedback sensors should be able to give very accurate and repeatable beam scanners.

Micro-mirror beam scanners suffer from the bowtie and cross-scan errors described in the polygonal scanners section. The intensity of the scanned beam is limited by the allowable temperature rise of the mirror material. 


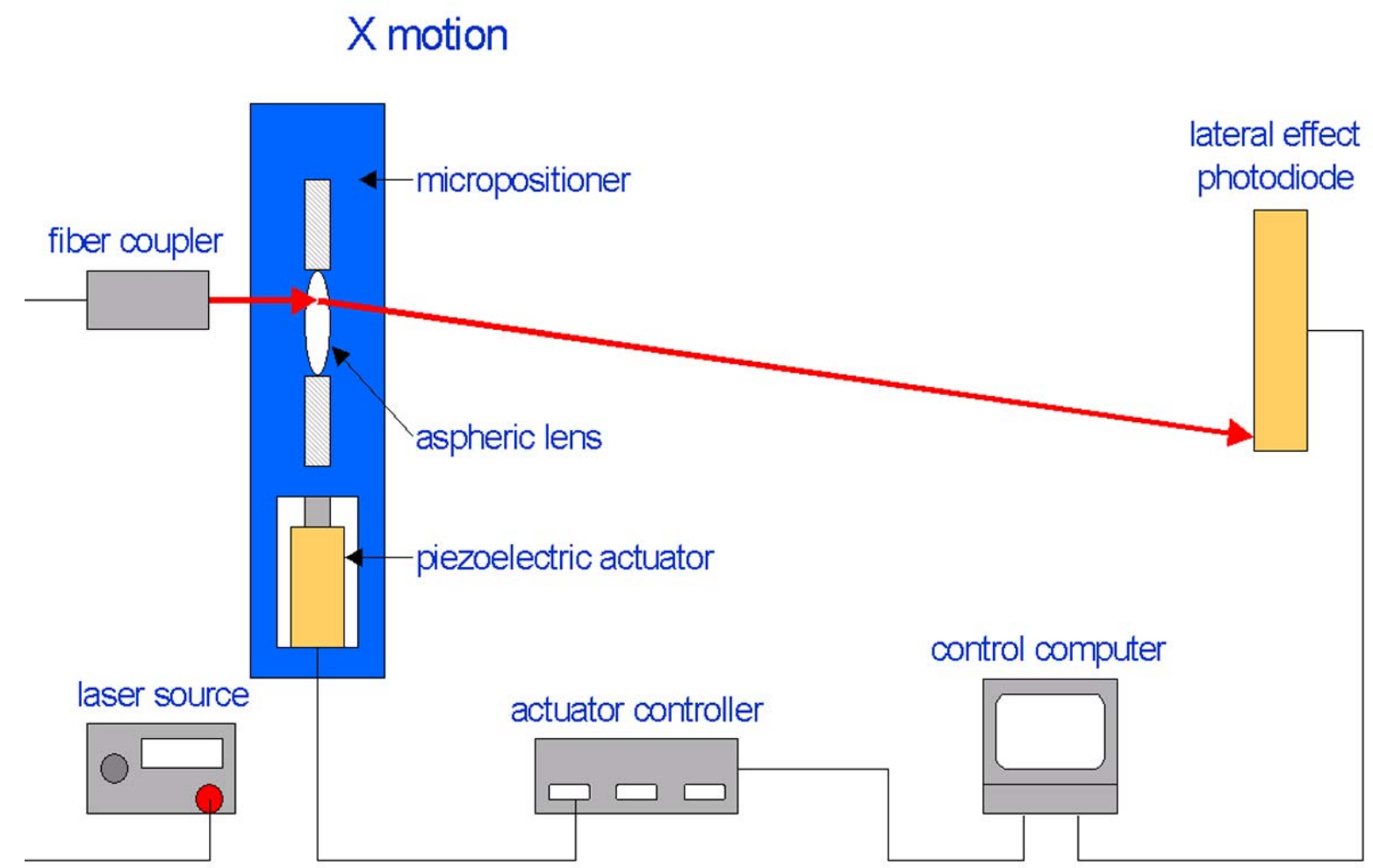

Figure 3.2.5. Micro positioner beam array scanner calibration setup

\subsubsection{Binary Micro Mirror Beam Arrays}

Binary MMAs can generate very fast beam scanners, but they have a scanning accuracy and repeatability problem that is difficult to solve. The commercial units that are currently available have no micro mirror displacement sensors, so it is difficult to know the azimuth and elevation angle of the beam at any particular moment of time. A calibration setup similar to the one depicted in Fig. 3.2.5 may be used to map angular displacement versus switching time for each micro-mirror, but there is no warranty that this map will not change during actual field operation.

\subsubsection{Micro Positioner Beam Arrays}

The mapping accuracy of a LADAR sensor depends on the accurate knowledge of the azimuth and elevation angles of the beam scanner. Unfortunately the scanning micromirror array and binary micro mirror array devices, which are currently commerciallyavailable, are not equipped with angular position sensors which can provide that information. In [Boone et al., 2002] we propose the use of a high performance micro positioner for a deep space communications laser scanner. Fig. 3.2.5 shows the schematic drawing of a calibration setup of such a scanner. A laser diode or fiber coupler is placed at the focus of a small refractive beam collimating lens. The lens is mounted on the moving stage of a 2D planar micro positioner, which can generate a beam 


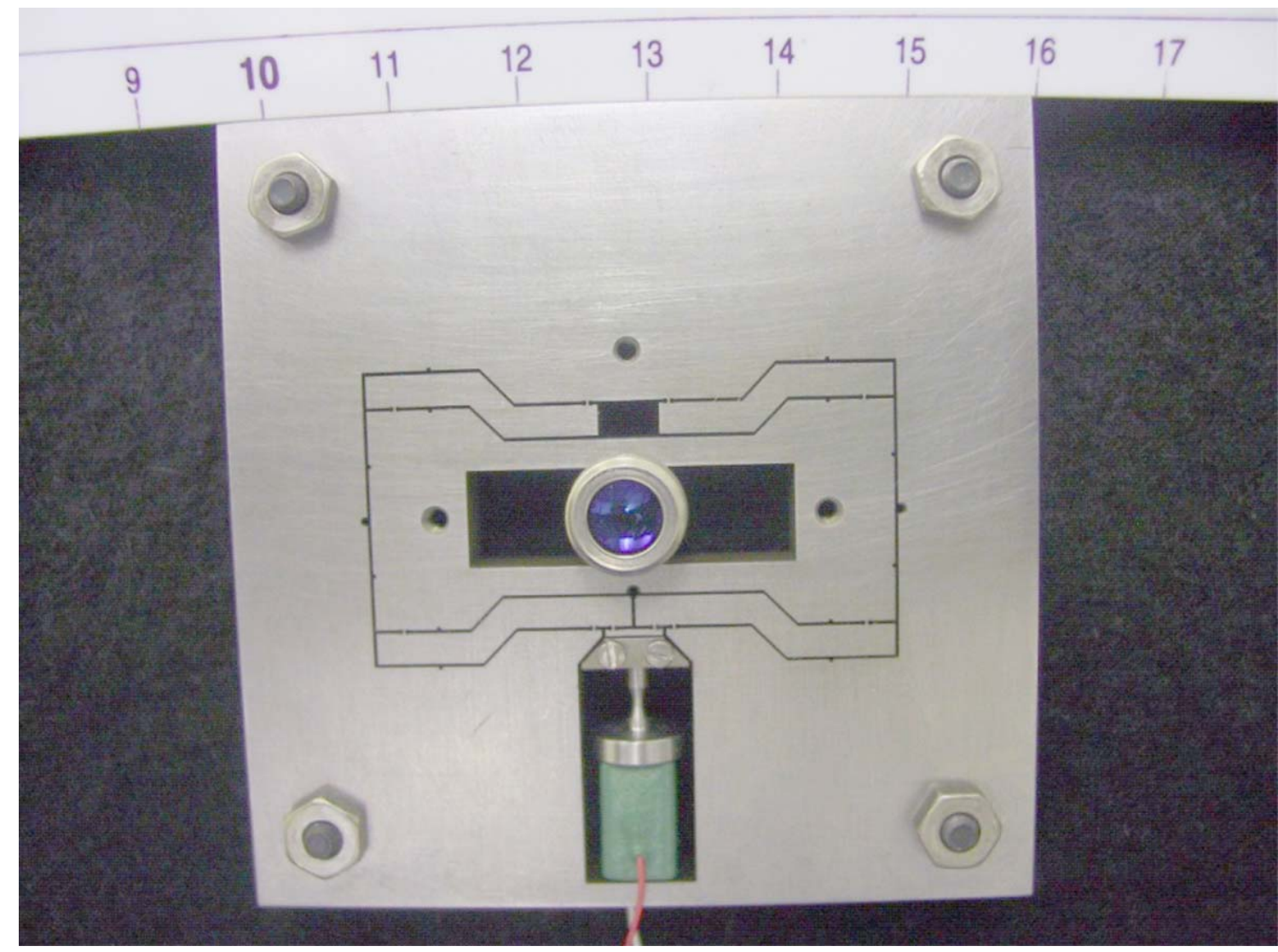

Figure 3.2.4. Credit card size Parallel Cantilever Biaxial Micro Positioner beam scanner.

azimuth and elevation angular displacement as the stage moves along two orthogonal $X$ and $Y$ axes. The calibration setup includes a 2D lateral effect photo diode, which measures the beam spot $X Y$ deviation. Fig. 3.2.6 shows the picture of a credit card size Parallel Cantilever Biaxial Micro Positioner (PCBMP) beam scanner being prepared for testing. PCBMPs have very low cross talk and angular deviation errors [Amatucci et al., 2000] and are ideal for high accuracy beam steering. An additional feature of PCBMPs is that they allow the displacement sensors to be aligned with the force actuator axis thus eliminating the effect of Abbe sine errors* ${ }^{*}$

To generate arrays of steerable laser beams [Dagalakis et. al., 2002] it is possible to build MEMS size arrays of PCBMPs with integrated laser beam sources and displacement sensors. In order to simplify the moving stage fabrication and to reduce mass it is possible to replace the refractive lens with a flat Fresnel lens, or a holographic optical element [Marshall, 1991], or mount the optical fiber on the moving stage. These arrays of micro positioner scanners can be built on chips that are mounted on sockets

\footnotetext{
* Abbe sine error is defined as the displacement error generated by the angular deviation error of a position sensor target, which is equal to sine(angular deviation error) $\mathrm{x}$ (distance between the actuator center line and the target location).
} 


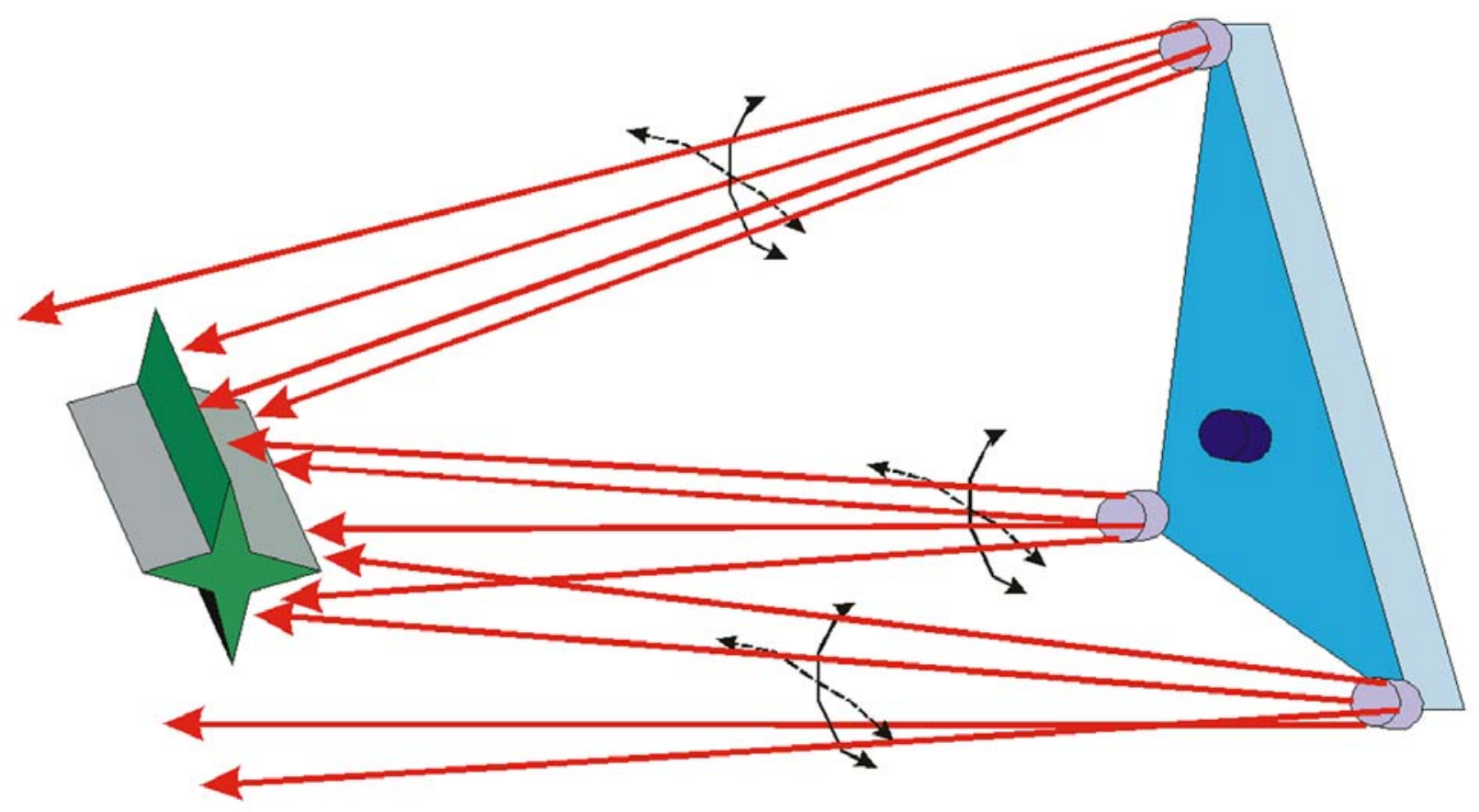

Figure 3.2.7. Multiple micro positioner scanners mounted on a triangular plate

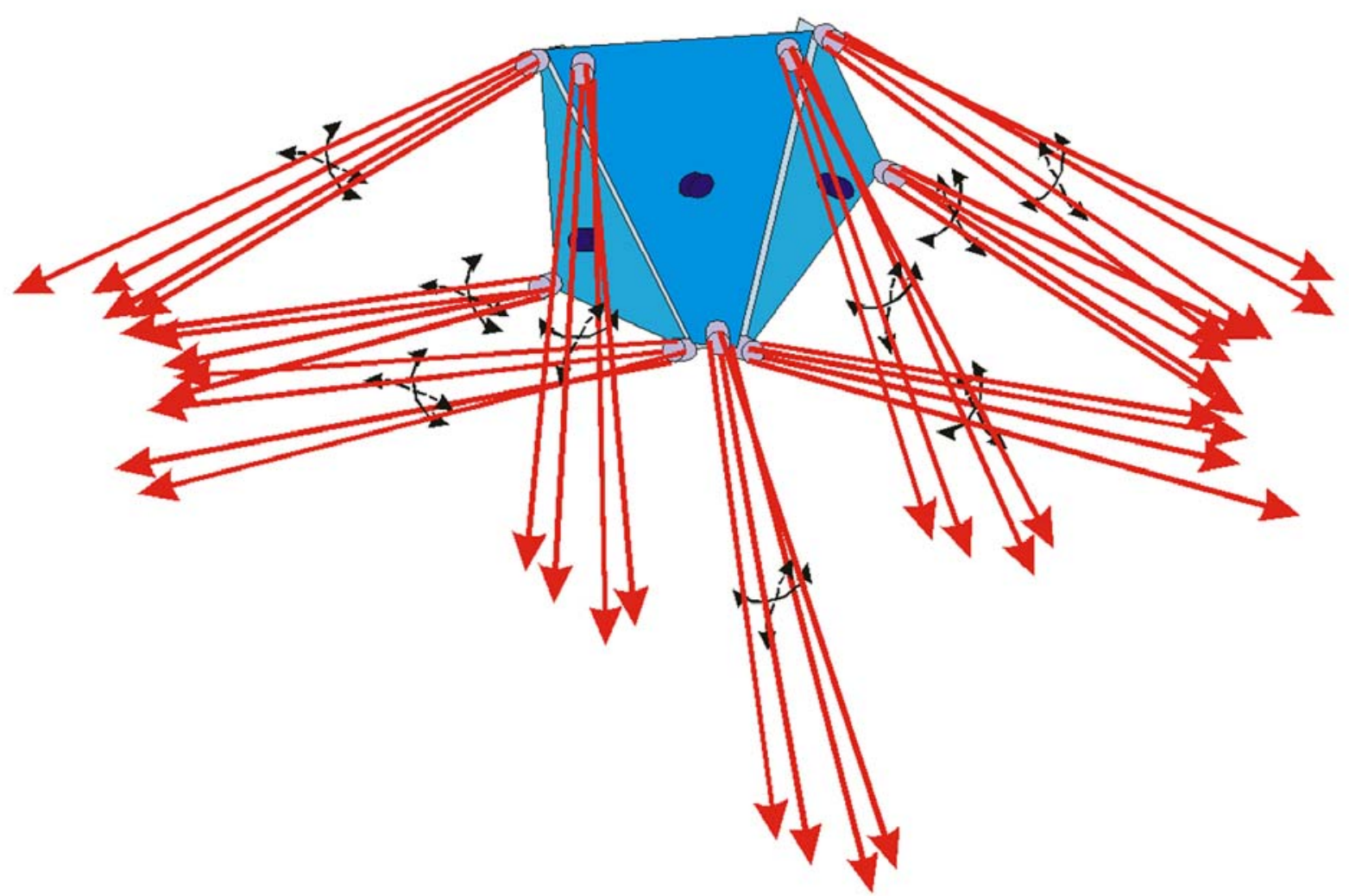

Figure 3.2.8. Multiple scanner plates mounted on a convex frame 
and can be located anywhere in the workspace. Because there is no need for external laser sources it is possible to use a convex or flat mounting plate. Fig. 3.2.7 shows an example of a triangular mounting plate for such sockets. Several of these plates can be mounted at different locations for the complete coverage of a wide workspace. Fig. 3.2.8 shows a convex frame supporting three such plates.

\subsubsection{Micro Crystal Beam Arrays}

An interesting recent development from the field of optoelectronics is the use of arrays of micro crystals for the control of laser beam direction. These arrays are used for optical switch routing [Trellis Photonics, 2004], but could perhaps find applications as beam scanners too. Similar to an acousto optic device these micro crystals use a voltage controlled Bragg grating to route optical beams for communication purposes. These switches do not rely on moving parts and thus can switch in 10 ns [McCarthy, 2001]. Like binary micro mirrors though the commercial products currently available are not equipped with beam deflection angle sensors and thus their accuracy and repeatability could vary with operating conditions.

\subsubsection{Thermo Optic Beam Array Switches}

Another technology for optoelectronic switching uses heat to control the laser beam direction. Bubblejet switches pioneered by [Agilent Technologies, 2004] rely on the generation of small bubbles that change the refractive index along an optical channel and divert a laser beam to another channel. The bubble is created by a micro heater inside a small cavity, which contains fluid that has an index of refraction identical to that of the optical channel. Arrays of these optical channels intersecting at precise angles allow the routing of several input beams to all the channels they intersect. A similar switch, which is using a small capillary partially filled with oil, was built by [NTT Electronics, 2004]. In another variation of the same basic technology a thermally actuated micro mirror optical switch was pioneered by [Nanovation Technologies, 2004]. All commercially available thermo optic beam array switches are binary devices with no beam deflection angle sensors.

\subsubsection{Liquid Crystal Beam Switches}

Liquid crystals can be used to rotate a laser beam polarization. Polarization beam splitters can then route the polarized beam in the desired directions. [Spectra Switch, 2004] is using this technology to build optical shutters and optical switches. Liquid crystal beam switches are binary with no beam deflection angle sensors. 


\subsection{Fast Beam Steering}

To date the fastest mechanical beam deflection mechanisms are rotating polygon mirrors and galvo-driven mirrors (see Figures 3.1 and 3.2). There are physical limits to how fast such systems can scan. In the case of rotating polygons the mirrors are supported as facets on a stable glass. Maximum rotation rate is generally given by [Montagu and DeWeerd, 1996] as:

$$
\dot{\omega}=\frac{1}{2 \pi r_{0}} \sqrt{\frac{\sigma_{u l t}}{\rho(3+\eta)}}
$$

where $r_{0}$ is the radius $(m)$ from the axis of rotation to the mirror facet; $\sigma_{\text {ult }}$ is the tensile strength of the mirror $\left(\mathrm{Pa}=\mathrm{kg}-\mathrm{m} / \mathrm{s}^{\wedge} 2\right)$ where the supporting structure is assumed as a solid; $\rho$ is the material density $\left(\mathrm{kg} / \mathrm{m}^{\wedge} 3\right)$; and $\eta$ the Poisson's Ratio for the support material. This generally limits angular rotation rates to around 1000 revolutions per second for glass and a few thousand revolutions per second for a high strength metal substrate; deformation of the mirror will further limit the achievable angular rotation rate. Commensurate scan rates can be as high as $50 \mathrm{kHz}$. However, the faster scan rates are generally achieved through the use of a greater number of polygon facets with a commensurate reduction in FOV. Furthermore, the movement (rotation) of the polygon is continuous; there is no means to stop and start it in a controlled fashion so as to permit, for example, re-targeting of the field of view.

Galvo scanners are presently limited to a 100 microsecond response time for a 0.1 degree discrete rotational step. Certain manufacturers have indicated that it may be possible to to move a galvo-driven mirror 0.01 degrees in 50 microseconds. Using the former figures, a 20 degree FOV could be scanned in $(20 / 0.1)^{\star} 100=20 \mathrm{~ms}$ yielding a scan rate of $50 \mathrm{~Hz}$ (200 degrees / s). When operated under open loop control and using a sinusoidal driving function, high end galvos can reach significant update rates for relatively small FOVs (see Figure 3.3.1).

Neither galvos nor rotating polygon mirrors lend themselves to mass production in small, compact devices at affordable prices of the type envisioned in this paper for NGL. As previously described, work is currently underway at NIST to investigate MEMS (micro electro-mechanical systems) concepts for beam steering using mirror systems with individual macro-scale mirror elements on the order of $1 \mathrm{~mm}^{2}$ and response times on the order of $1 \mathrm{~ms}$ (i.e. these are much smaller than normal galvo mirrors, but their size is such that even with piezoelectric actuation their peak response time is on the order of milliseconds, not microseconds. The smaller the mirror the smaller the mass moment of inertia and thus the faster achievable mirror acceleration. 


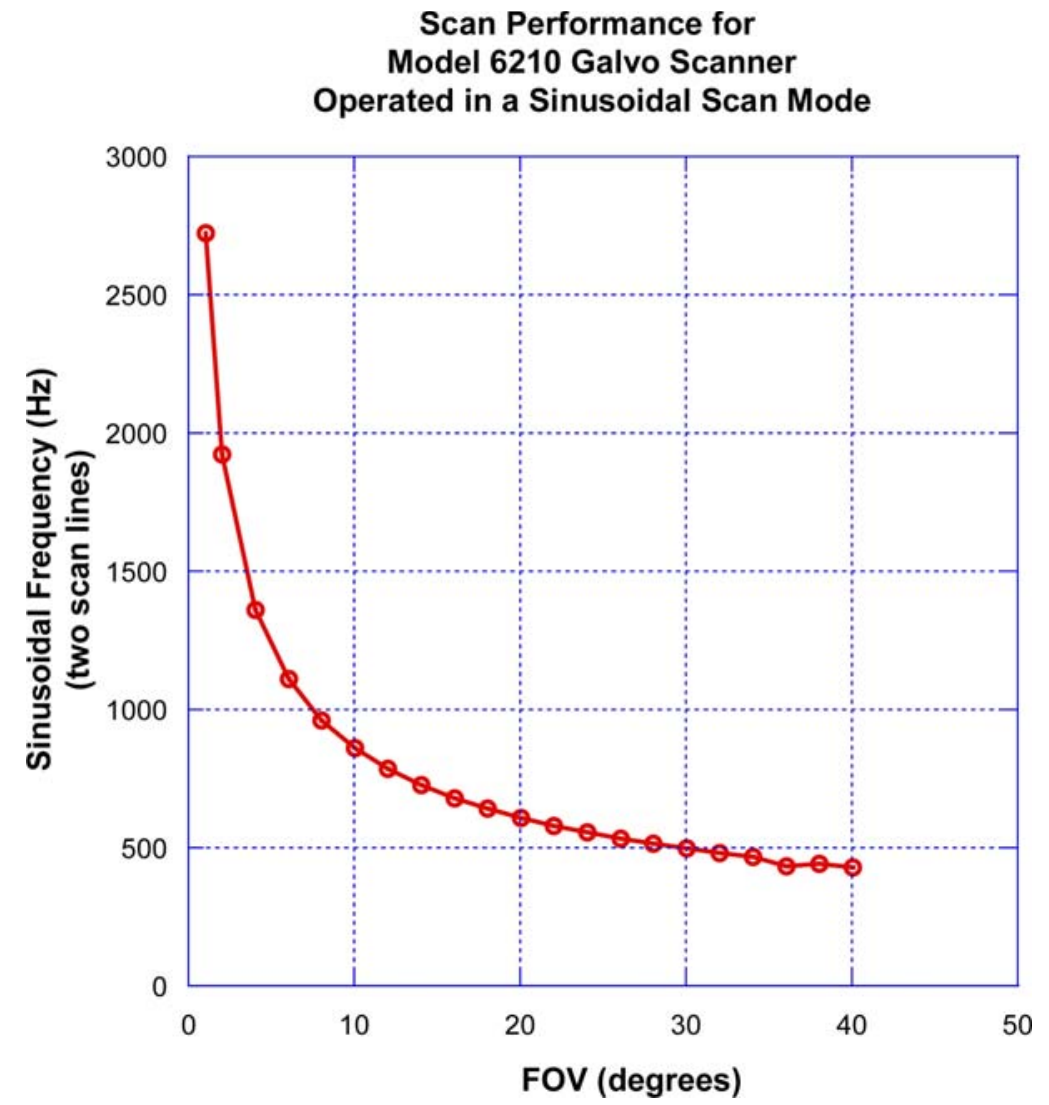

Figure 3.3.1. Typical performance envelope for a galvo scanner operating in a continuous sinusoidal oscillation mode.

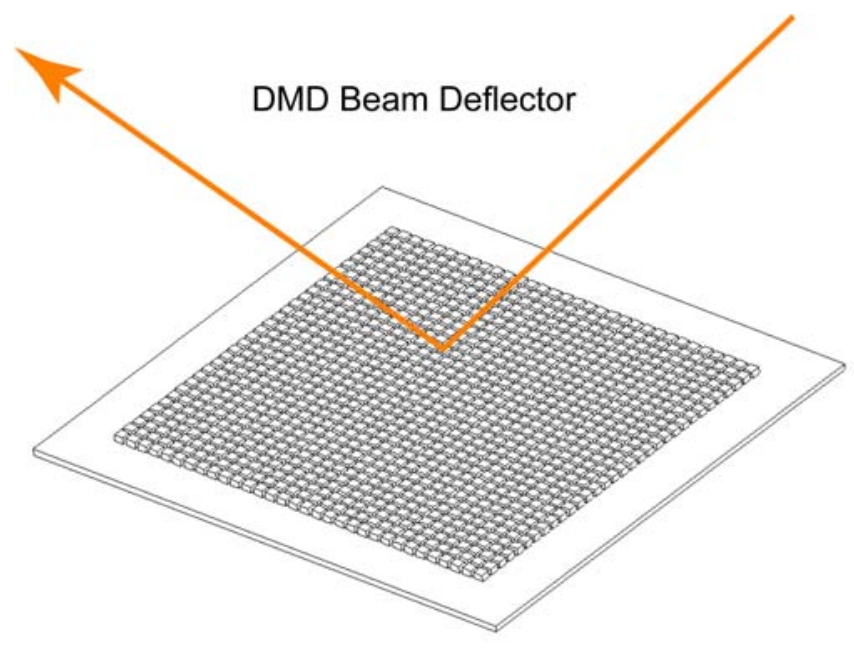

The FANDANGO (Fast ANgular Deflection At Nist.GOv) experiment currently underway seeks to solve the fast mirror problem by using planar arrays of mirrors that that have individual mirror dimensions on the order of $10 \mu \mathrm{m}$ on a side, with correspondingly faster angular response. Presently, the FANDANGO experiment makes use of a TI 0.7 XGA DMD (digital mirror device) with $1024 \mathrm{x}$ 768 binary mirrors, $13.7 \mu \mathrm{m}$ $\times 13.7 \mu \mathrm{m}$ mirror patches, +/- $12^{\circ}$ mirror tilt, $24 \mu \mathrm{s}$ cycle time, $80 \%$ optical fill factor, and $10 \mathrm{~W} / \mathrm{cm}^{2} \max$ irradiance.

The FANDANGO experiment uses a fast $0-D$ LADAR as its core distance measuring device. The unit operates at $780 \mathrm{~nm}$ with a $22 \mathrm{~mW}$ illumination power and is capable of acquiring range measurements at a rate of $625^{\star} 10^{3}$ samples per second. The exit beam diameter $(3.5 \mathrm{~mm})$, divergence angle (0.1 mrad), and power were selected for compatibility with the afforementioned DMD deflector.

Figure 3.3.2. DMD micro deflector array concept; each of 786,432 MEMS mirrors are individually addressable. 


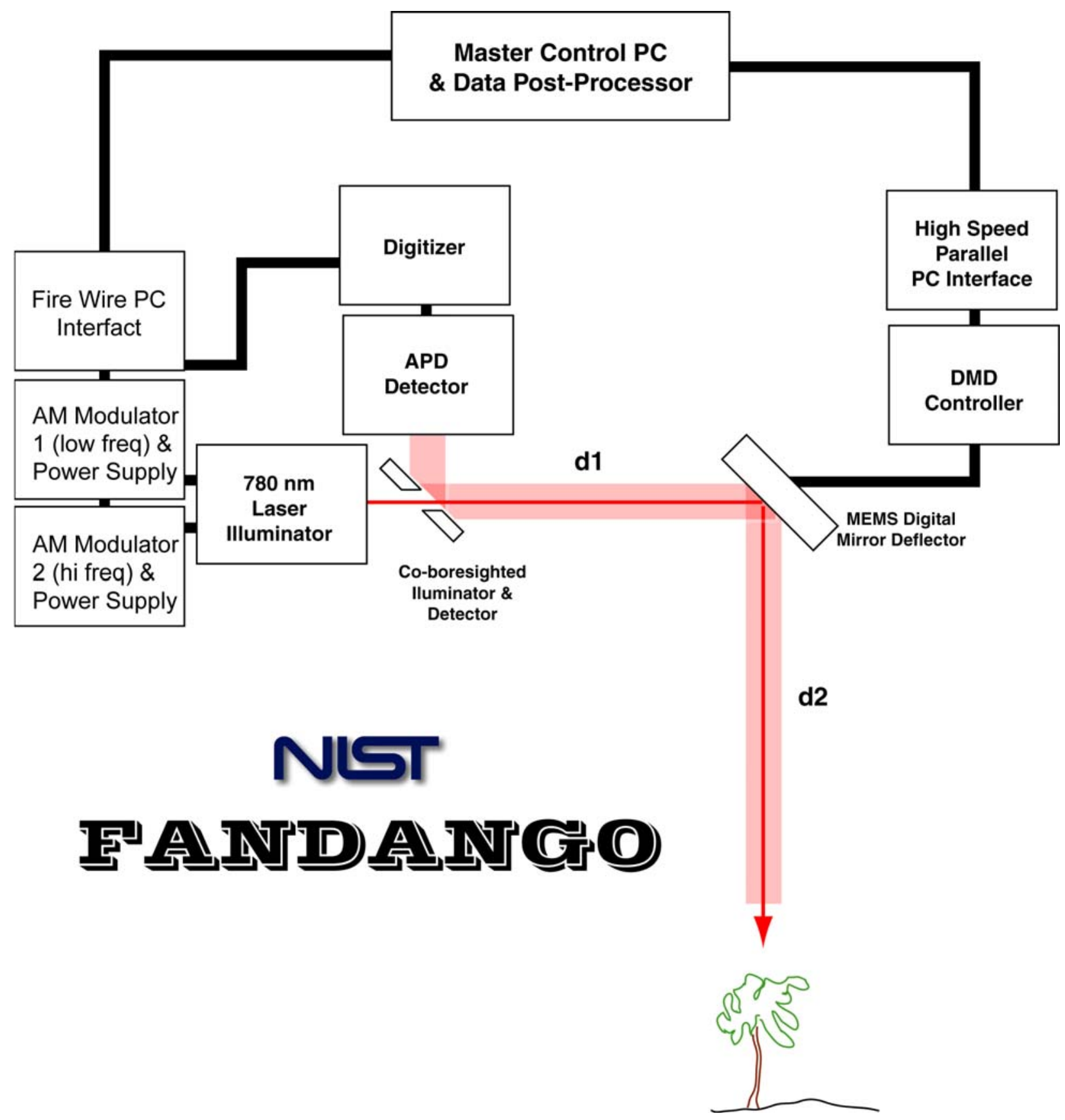

Figure 3.3.3. NIST FANDANGO experiment architecture. 


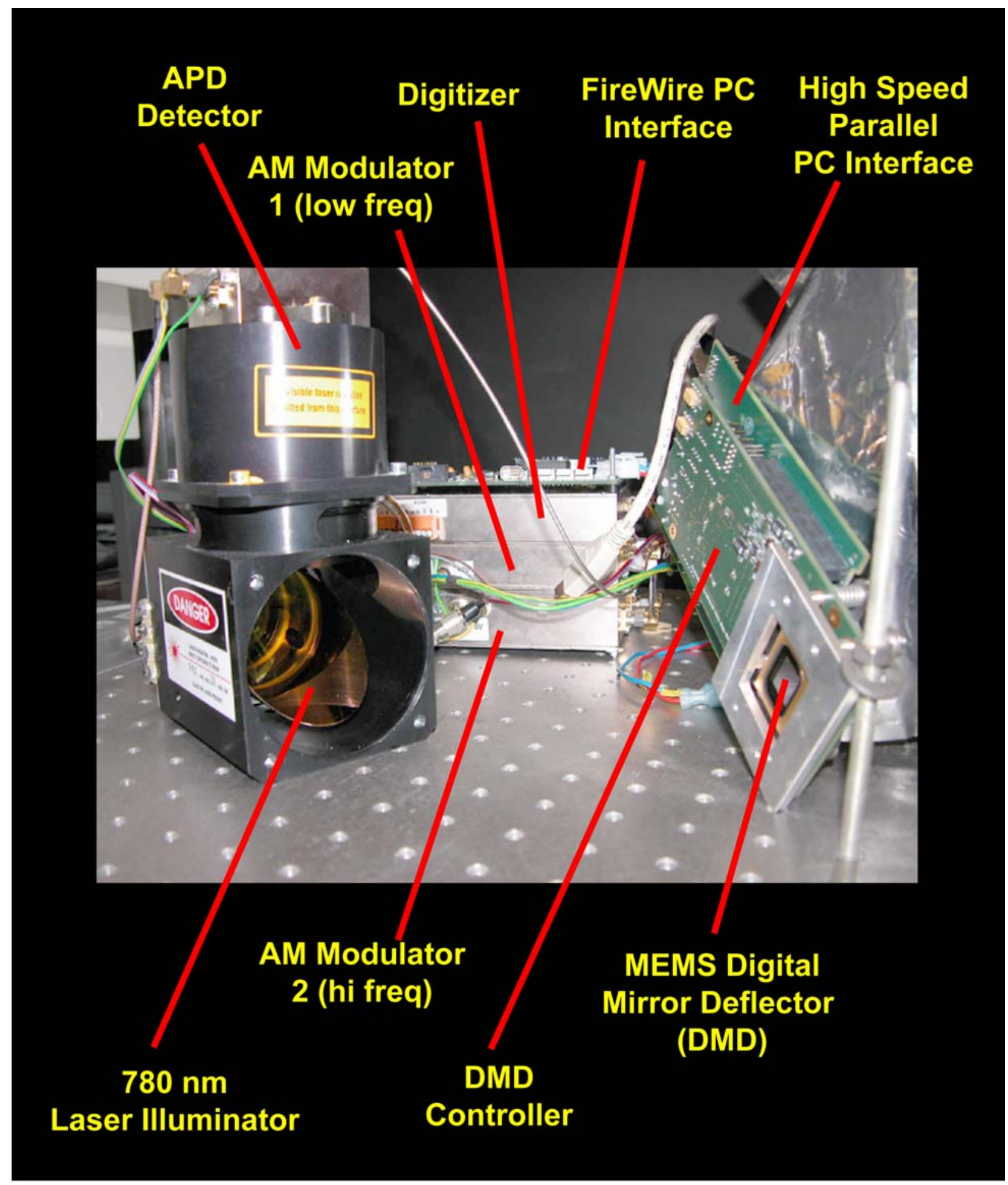

Figure 3.3.4. NIST FANDANGO experiment showing core 0-D dual frequency AM ladar and MEMS digital mirror deflector. Control and interface electronics are shown in the background. High speed (Fire Wire) interfaces to a local fast PC (64 bit bus) provide for LADAR and DMD control and data offload. 


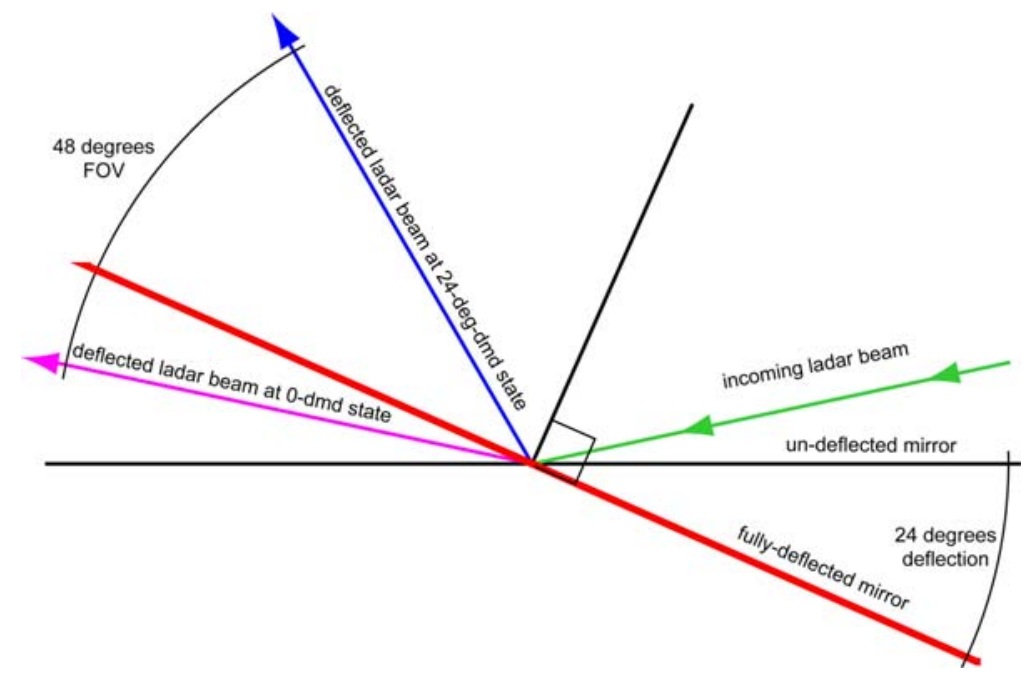

Figure 3.3.5. FOV determination for the NIST FANDANGO experiment

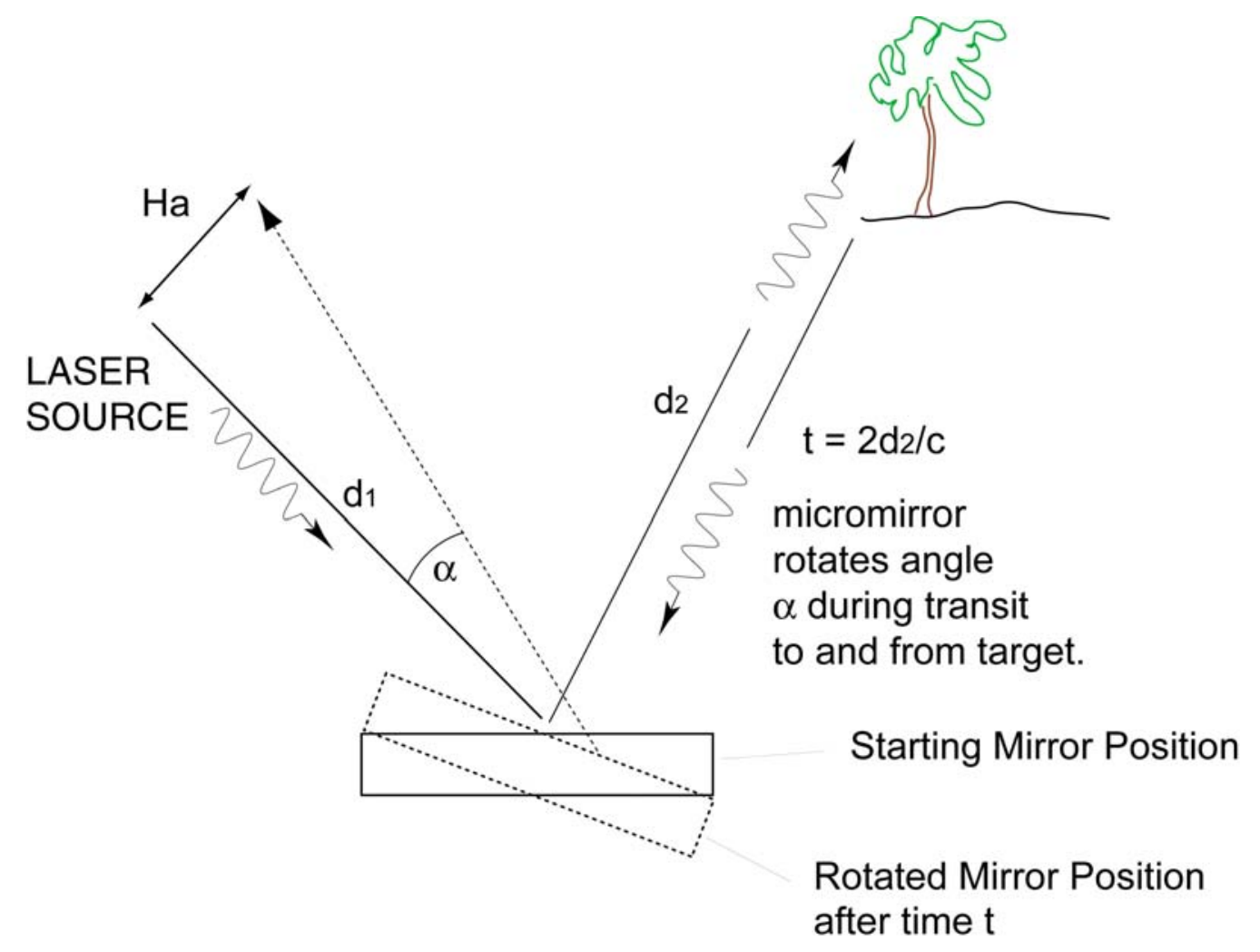

Figure 3.3.6. Determination of minimum receiver aperture size for FANDANGO. 
The receiver aperture requirements were determined as follows. The angular deflection rate of the DMD, based on binary state specifications $\left(+/-12^{\circ}\right.$ in $\left.24 \mu \mathrm{s}\right)$ is:

$$
\dot{\alpha}=\left(\frac{2 \cdot 2 \cdot 12}{24 \cdot 10^{-6}}\right)=2 \cdot 10^{6} \frac{\text { deg rees }}{s}
$$

The additional " 2 " in the numerator accounts for the doubling of the deflection angle of the laser beam since the incident and reflected angles must be the same (see Figure 3.3.5). The time delay, which accounts for the round-trip time, is:

$$
\tau=\frac{2}{c}\left(d_{1}+d_{2}\right)
$$

where $d_{1}$ and $d_{2}$ are the distance from the laser source to the DMD mirror and the distance from the DMD mirror plane to the target, respectively, as defined in Figure 3.3.6 below.

There is a point at which the scanning angular deflection rate can in fact be too fast this occurs when the detector aperture radius, $\mathrm{H}_{\mathrm{a}}$, is not large enough to capture the returning light bearing the range data.

In the time it takes for the beam to reach the target and return to the detector, the deflector mirror will rotate an angle, $\alpha$, given by:

The required aperture radius, with all units in meters, is given by:

$$
\alpha=\tau \cdot \dot{\alpha}
$$

which can be simplified to:

$$
\begin{array}{r}
H_{a}=d_{1} \cdot \sin \left(\frac{2 \pi \alpha}{360}\right) \\
H_{a}=d_{1} \cdot \sin \left(0.74 e^{-4} \pi d_{2}\right)
\end{array}
$$

Figure 3.3.7 shows a plot of this value as a function of the laser to DMD mirror distance (d1) and the target range (d2). For the purpose of NGL concept development, the minimum value of $\mathrm{d} 2=50 \mathrm{~m}$, although $100 \mathrm{~m}$ would be preferable for a number of 
machine control applications. The current receiver aperture for the core LADAR used in FANDANGO is $50 \mathrm{~mm}$ so the angular rotation rate of $2(10)^{6}$ degrees/s is accomodated and preliminary lab bench results support the achieving of scan data at this rate.

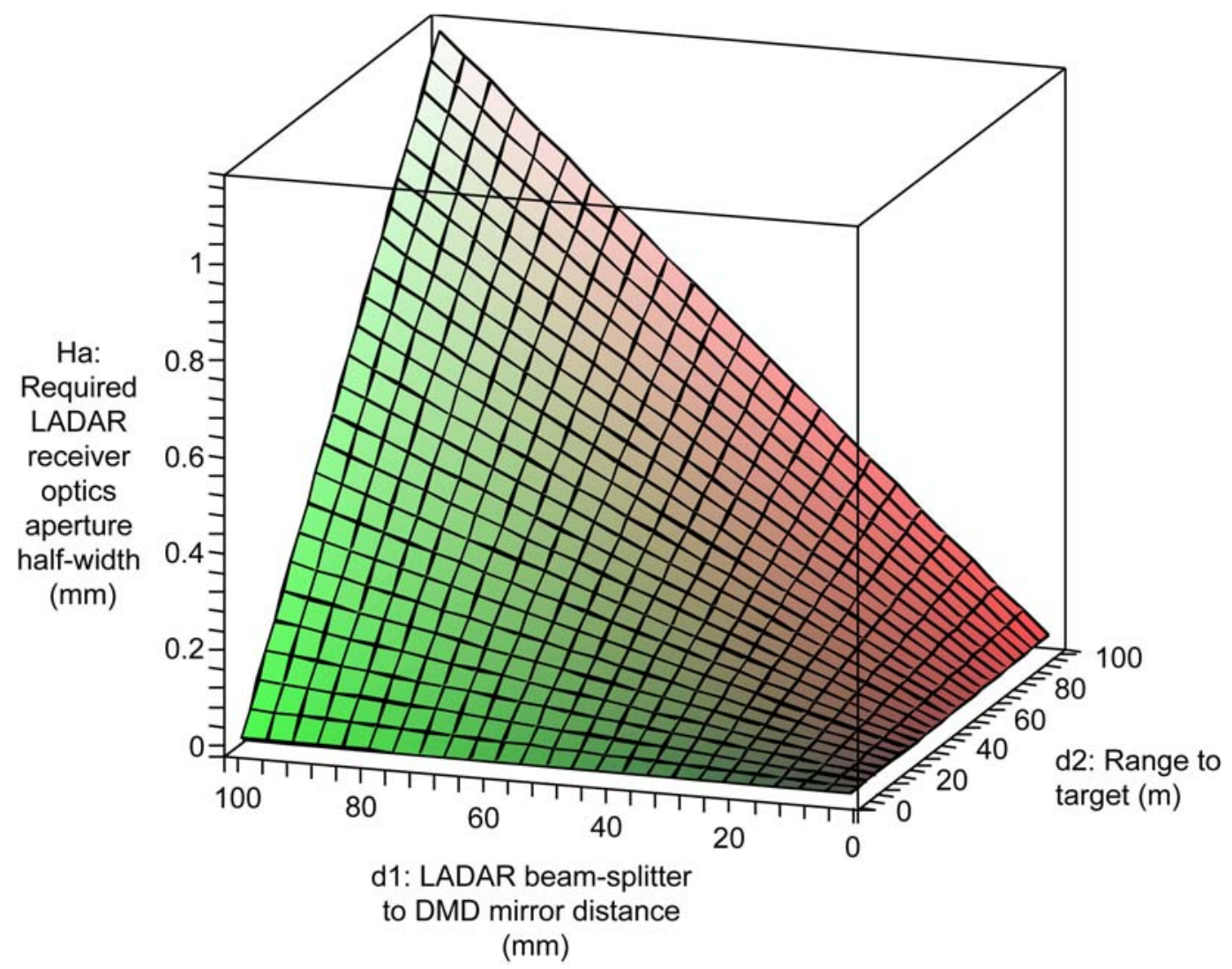

Figure 3.3.7. Required detector apertures for various DMD beam steering options. 


\subsection{Risley Prisms}

Optical stages for microscopes have for some time been optionally equipped with Risley prisms for the purpose of directing the field of view over the object under study. This same concept has been implemented in at least two LADAR systems. Risley prisms consist typically of circular-cut lenses in which the thickness varies in a planar fashion from one side to the other. For scanning operations these are customarily operated in pairs that are co-boresighted and axially aligned. Scanning is accomplished by counter-rotating the two prisms with respect to one another and the speeds of individual rotation of each prism in the pair can be different in order to achieve a desired scan "field of regard" (FOR). This is of particular interest in the area of staring array (FPA) LADAR, since these devices commonly have relatively small (on the order of a few mrad) FOVs and require scanning mechanisms to make them of practical use.

Figures 3.4.1 and 3.4.2 provide examples of a system recently implemented by MIT Lincoln Labs as part of their contribution to the Jigsaw program. The FPA (a 32×32 pixel Geiger-mode device) had a native FOV of about $10 \mathrm{mrad} \times 10 \mathrm{mrad}$. The clear aperture for each of the Risley prisms was $75 \mathrm{~mm}$ with a maximum individual prism thickness of $10 \mathrm{~mm}$. These are driven by a planetary gearing system as shown in the upper right portion of Figure 3.4.1.
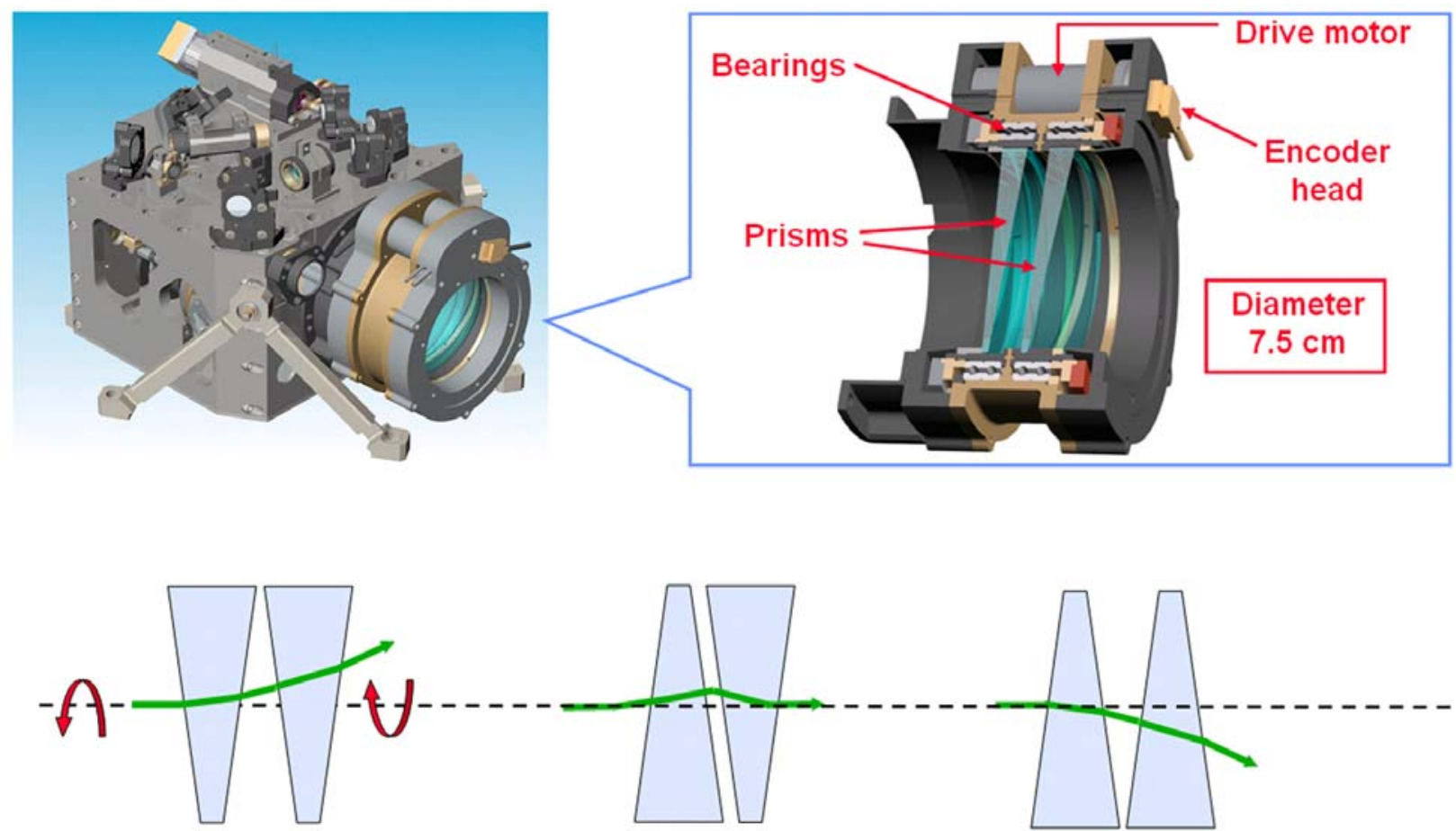

Figure 3.4.1: Implementation of a Risley prism scanner for an FPA staring array LADAR. Courtesy MIT Lincoln Lab. 
In the Lincoln Lab approach the prisms were operated at a constant angular rate to reduce power with one prism rotated at $36 \mathrm{~Hz}$ and the other at $28 \mathrm{~Hz}$. This difference produces a spirograph pattern with the scanned areas comprising pedal-like apendages to a central locus. The entire pattern precesses due to the different angular rotation rates of the two prisms, thereby assuring complete coverage of a circular field of regard (FOR) that is much larger than the native FOV (see Figure 3.4.2, lower right). For the device tested the FOR was approximatey 11 degrees -- still quite small by the standards of scanning LADARs. The advantages are relative compactness in the scanning hardware.
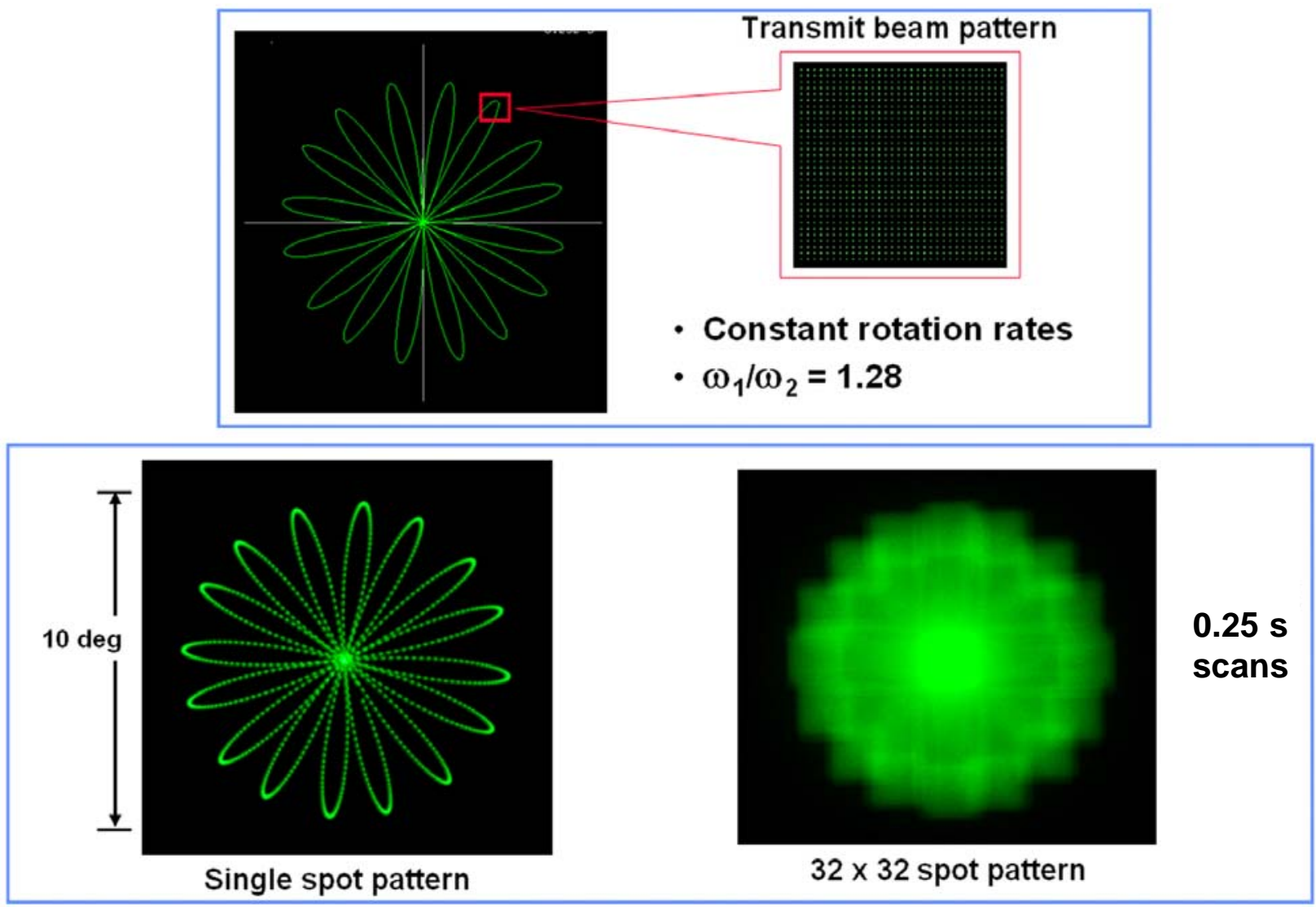

Figure 3.4.2: Scan pattern field of regard (FOR) for a Risley prism scanner for an FPA staring array LADAR. Courtesy MIT Lincoln Lab. 


\subsection{Measurement Limitations Imposed by the Refractive Index of Air}

\subsection{Refractive Index: General considerations}

The index of refraction of air comes into play for all high-precision optically based measurements. As a general rule it is a relatively small effect, not of paramount importance for common measurement needs in the construction industry, but it plays a pivotal role in defining the accuracy of high-end measurements employing the most precise instruments available (such as the MetricVision ${ }^{\mathrm{TM}}{ }^{*}$ system described in the Appendix, or interferometer-based laser-tracker systems). Refractive index effects are a major limitation in ultra-high precision surveying or in the most demanding construction projects, including applications such as:

- Maglev rails, where desired specifications on the rail straightness are beyond current measurement capabilities;

- The Large Millimeter Telescope (currently under construction atop Sierra Negra in Mexico), which requires control of dimensions at the micrometer level over distances up to $50 \mathrm{~m}$; and

- Linear accelerator alignment, with needs for sub-millimeter measurements over distances on the order of $1 \mathrm{~km}$.

Uncertainty in refractive index is the ultimate limiting factor in dimensional measurements outside of a controlled, laboratory environment.

The refractive index is primarily important in two ways:

(1) For ranging systems, the range measurement depends on the velocity of light, hence on the refractive index. The propagation speed $v$ of a pulse through the atmosphere is $v=c / n_{g}$, where $c$ is the speed of light in vacuum and $n_{g}$, the group refractive index of air, is about 1.0003 under normal conditions. The group refractive index $n_{g}$ determines the speed of pulse propagation, while the phase refractive index $\mathrm{n}$ (usually referred to simply as refractive index) provides the proper wavelength correction for distance measurement with single-frequency interferometry.

(2) Variations in refractive index perpendicular to the line of sight refract transmitted light, displacing the image of the object being measured. This will give rise to angular errors in optical measurement systems. Errors in vertical angular measurement are usually a greater problem than in horizontal measurement.

\footnotetext{
* Commercial equipment and materials are identified in this paper as examples of certain types of instruments. In no case does such identification imply recommendation or endorsement by the National Institute of Standards and Technology, nor does it imply that the materials or equipment identified are necessarily the best available for the purpose.
} 
We may also distinguish between turbulent temperature fluctuations and larger-scale persistent thermal gradients arising from local sources of heating. When measuring distances on the order of tens of meters, we estimate that turbulent effects represent a somewhat smaller danger to measurement accuracy than do gradients arising from the worst sources of local heating, but both effects are significant. Turbulence is discussed in Section 4.6.

The air refractive index is primarily a function of atmospheric pressure, temperature, and humidity. For visible or near-infrared light, variations in the refractive index over modest distances are primarily a function of temperature gradients, with only small additional contributions from humidity and pressure variations. In the visible spectrum, a $1^{\circ} \mathrm{C}$ increase in air temperature decreases the refractive index by a little less than $1 \times 10^{-6}$. Using this fact, we can estimate the likely errors due to refractive index effects, both ranging errors and angular errors. These sources of uncertainty, and methods for avoiding these errors, are discussed in the following sections.

\subsection{Ranging Errors: Magnitude of the Problem}

High-accuracy range measurements usually correct for the refractive index by measuring atmospheric pressure, temperature, and possibly humidity. Based on these measurements, the refractive index can be computed using the Ciddor equation [Ciddor 1996] or one of several other equations that relate these parameters to the refractive index. The accuracy of the correction depends on (1) the accuracy of the sensors and (2) how well the air sampled by the sensors is representative of the measurement path. Sensor errors that will cause a $1 \times 10^{-6}$ relative error in range measurements include:

- an error of $1^{\circ} \mathrm{C}$ in temperature measurement

- an error of $400 \mathrm{~Pa}$ in pressure measurement

- an error of $90 \%$ in relative humidity measurement at $25^{\circ} \mathrm{C}$.

The primary source of concern is that a local measurement of temperature is not representative of the average temperature along the measurement path. (Variations in pressure may also be an issue if measuring vertically; a height measurement of $100 \mathrm{~m}$ would be in error by $0.16 \mathrm{~mm}$ if refractive index were calculated using the pressure at one endpoint.) Some of the worst potential errors in temperature measurement can be avoided if suitable care is taken. Temperature sensors must be shielded from direct heating by the sun and should not be placed too near localized sources of heat. This includes heat generated by the measurement equipment itself; a poorly designed system with sensors mounted directly on the equipment is unlikely to achieve high accuracy, or a temperature sensor accidentally placed near heat exhaust from the measuring electronics can easily give errors in range measurements of several parts per million. Rüeger [1996] recommends that temperature sensors should be placed at least $1.5 \mathrm{~m}$ away from any object-including the ground or people. 
When a relative uncertainty smaller than about $2 \times 10^{-6}$ is required (that is, better than $20 \mu \mathrm{m}$ on a $10 \mathrm{~m}$ length), it may be necessary to employ special equipment, as described below, in order to insure that the average temperature along the measurement path is compensated correctly.

\subsection{Correcting range measurements for refractive index in regions of varying air temperature}

There have been several methods proposed to compensate for possible temperature variations along the measurement path. The most straightforward method is to use a series of temperature sensors strung out along the measurement path, but this is usually impractical. Two other methods, both of which directly compensate for temperature variations integrated along the entire measurement path, have demonstrated promise.

(1). Two-color techniques based on dispersion have been studied since the 1970's. The difference in refractive index for two different wavelengths in air depends linearly on air density, as does the refractivity of the air. (Refractivity is $n-1$, where $n$ is the refractive index.) Thus, by measuring quantities related to the difference in refractive index for two wavelengths, it is possible to deduce the index of refraction if the air composition (and hence the dispersion) is known. If air composition varies - due to variations in humidity, for example - then three or more colors of light must be used to solve for the unknown composition. For example, see [Huggett and Slater, 1975] where a microwave beam is used to account for humidity variations.

When an unknown distance is measured twice, using two different wavelengths of light, the two results will differ if no correction is made for dispersion. The difference in the two results provides a direct measure of the dispersion for the two wavelengths. From this difference and the known dispersive properties of air, the refractive index at either wavelength, integrated along the measurement path, can be deduced. The challenge in implementing a practical system based on this principle is that the dispersion is very weak and consequently it is difficult to perform the measurement sufficiently well to achieve useful results. Over the entire visible spectrum the refractivity varies only by about $2.5 \%$ or $1 / 40$. This factor represents a de-magnification of sensitivity, requiring that the precision of the individual measurements in the two colors must be 40 times greater than the meaningful precision of the result. In other words, errors in measuring the two individual lengths that are not common to both measurements are multiplied by 40 in the final result; to achieve an uncertainty below $1 \mu \mathrm{m}$ it is necessary to compare the two length measurements with non-common-mode errors below $25 \mathrm{~nm}$ when visible light is used, or to achieve a relative uncertainty below $1 \times 10^{-6}$ it is necessary to compare the two length measurements at a level better than $2.5 \times 10^{-8}$. This situation is improved somewhat by using one color in the ultraviolet (where dispersion is greater), and this approach is used in some commercial instruments. 
In addition to the work of Huggett and Slater, early work on multi-color systems includes studies by [Levine, 1984] and the development of a commercial instrument, the Terrameter. The Terrameter could measure a length $L$ with an uncertainty on the order of $0.3 \mathrm{~mm}+0.12 \times 10^{-6} \mathrm{~L}$, as reported by [Langbein et al,1987].

A particularly interesting recent implementation of the two-color technique was commercialized by Sparta, Inc [Lis, 1995]. This product as it currently exists was developed for the semiconductor industry and is too costly to be practical for all but high-end applications, but the basic measurement scheme is clever and has been shown to yield excellent results. The Sparta system frequency-doubles a portion of a laser beam into the ultraviolet, and the resulting two-frequency beam traverses the measurement path of interest (see figure 4.3.1). After traversing the measurement path, the two beams pass through a phase modulator and a second frequency doubler. In the second doubler, a portion of the beam at the original frequency is doubled, bringing it to the same frequency as the ultraviolet that traversed the measurement path, and these two equal-frequency beams interfere on a detector. (Some of the ultraviolet beam from the measurement path is also doubled a second time in the second doubler, but this light is discarded.) The phase of interference on the detector can be determined with the help of the phase modulator.

In the absence of dispersion, the two common-path beams would remain precisely in phase as they propagate along the measurement path, but in the presence of air the phase of the frequency-doubled ultraviolet beam lags behind that of the visible beam because of the larger refractive index in the ultraviolet. The phase shift observed in the final interfering beams is a very direct and accurate measurement of dispersive effects. The Sparta instrument achieves order-of-magnitude reductions in turbulence errors over short distances in an environment characteristic of turbulence inside a stepper used for integrated circuit manufacture. The two-color correction procedure reduces turbulence errors from $30 \mathrm{~nm}$ to less than $3 \mathrm{~nm}$.

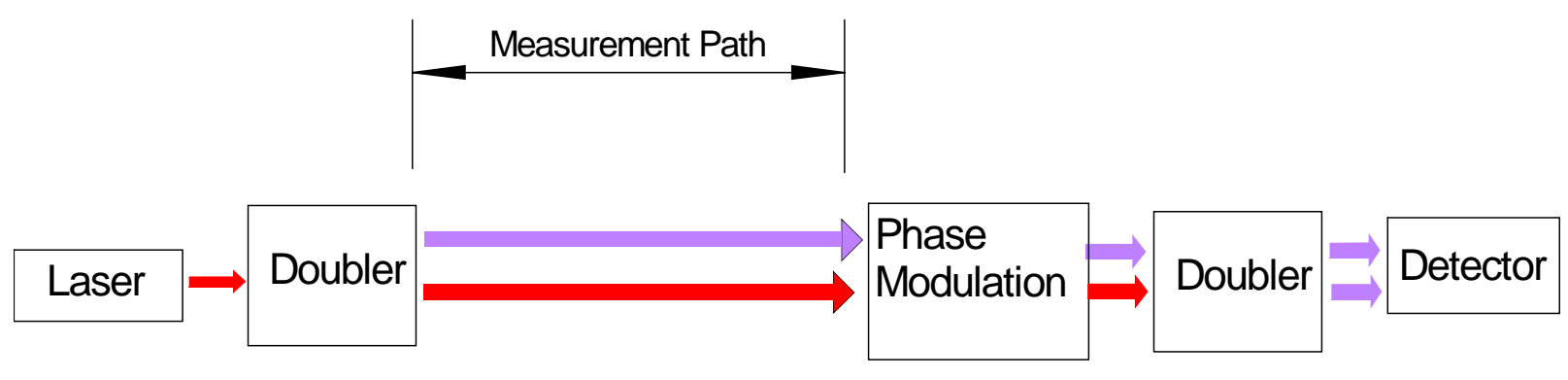

Figure 4.3.1. Principle of second-harmonic two-color interferometry. The frequency-doubled beam is shown above the non-doubled beam in the diagram, but in reality the two beams spatially overlap except inside the phase modulator (where they are separated, modulated, and recombined). 
The essential ideas behind this frequency-doubling technique were first demonstrated by Hoph et al [1980] and further developed by Ishida [1989].

Frequency-doubling is also the basis of another recent implementation of the dispersion method as described by Minoshima et al. [2000]. Minoshima's technique is based on time-of-flight ranging measurements carried out using two colors. Because it is a time-of-flight ranging system, this technique directly measures group refractive index whereas the Sparta interferometric system described above measures phase refractive index (from which group refractive index could be calculated if needed). Minoshima employed a femtosecond mode-locked laser for the ranging measurements. The rapid rise time of the femtosecond pulses makes it relatively easy to achieve a robust measurement capability at the $50 \mu \mathrm{m}$ level, and the high power in the pulses is efficiently frequency doubled. The difference in time-of-flight for the fundamental and frequencydoubled pulses depends on dispersion in the group refractive index, from which the actual refractive index can be inferred as before. As with all time-of-flight techniques, it is difficult to achieve a low relative uncertainty for measurements at shorter distances, and even over a long measurement path (240 $\mathrm{m}$ in a tunnel) Minoshima achieves only a very modest accuracy of $8 \times 10^{-6}$ in his refractive index measurement. However, the technique shows some promise for the future.

Another method for determining the integrated refractive index is to use sound-velocity measurements. Acoustic methods are potentially very attractive because the equipment is orders-of-magnitude less expensive than the more complicated optical methods. It has been recognized for some time that acoustic measurements might provide a good method for refractive index compensation in non-uniform environments, but to our knowledge there has been no careful design and testing of an acoustic system until the very recent work described by [Mihaljov, 2001] and [Lassila and Korpelainen, 2003]. This new system has not been tested in a field environment, but laboratory tests have given impressive results.

The underlying principles exploited by an acoustic system are as follows. For an ideal gas, the speed of sound is proportional to $T^{1 / 2}$ where $T$ is the absolute temperature. The speed also depends on composition of the gas (specifically, the molecular weight and the ratio of specific heats $\gamma$ ). For stationary air of uniform composition, the timeof-flight of a sound pulse over a known distance then provides a good measure of the temperature profile integrated along the path, in the approximation that the sound velocity varies by only a small amount so that the path integral can be linearized.

Near $20^{\circ} \mathrm{C}$ the speed of sound increases by about $0.6 \mathrm{~m} / \mathrm{s}$ (a fractional change of $0.17 \%$ ) for each $1^{\circ} \mathrm{C}$ increase in temperature. The same temperature increase will decrease the wavelength of visible light fractionally by $1 \times 10^{-6}$. Thus, if we are to compensate for refractive index with a relative uncertainty below $1 \times 10^{-6}$, it will be necessary to measure the speed of sound with an uncertainty below $0.6 \mathrm{~m} / \mathrm{s}$. To measure 
the speed of sound, it is necessary to accurately determine the time of flight for a pulse over a known distance. If the acoustic measurement path is equal to the optical interferometer's measurement path plus a known, fixed offset, then the distance for the time-of-flight measurement can be inferred from the optical interferometer reading.

Attenuation of ultrasound puts some limits on system performance. Ultrasonic waves are strongly attenuated in moist air; at a frequency of $40 \mathrm{kHz}$ the attenuation can exceed $1 \mathrm{~dB} / \mathrm{m}$ and the attenuation increases rapidly as the frequency increases beyond $50 \mathrm{kHz}$. In addition to the problem of loss of signal, the high frequency attenuation will limit signal rise times to values of about $10 \mu \mathrm{s}$ or more. In the absence of a sharp leading edge, the task of determining the time of arrival of a pulse is potentially complicated by reflected signals superimposed on the desired signal. The ultimate achievable uncertainty of the time measurement may then be limited by both the cleverness of signal processing and by good design of the acoustic transponders so as to send out and receive waves in a narrow angular range (reducing multipath reflected signals and increasing signal strengths at long distances).

A wind velocity of $5 \mathrm{~m} / \mathrm{s}$, blowing parallel to the measurement direction, would shift the apparent speed of sound by $5 \mathrm{~m} / \mathrm{s}$, an order of magnitude more than the desired uncertainty. Therefore it is necessary to measure bi-directional or round-trip flights so as to reduce the effect of wind. Simultaneous bi-directional measurements are somewhat superior to a sequential round-trip measurement when the wind is gusting and wind speed is changing rapidly. In principle, bi-directional measurements make it possible to determine the wind velocity and to make second-order corrections that are more accurate than simply considering the average round-trip speed. (In practice, second-order effects are rather small for normal wind velocity but increase quadratically with wind speed, reaching an error of $0.6 \mathrm{~m} / \mathrm{s}$ only at a rather high wind speed of $15 \mathrm{~m} / \mathrm{s}$. A transverse wind will also produce second-order effects, but only half as large as the second-order correction for wind along the measurement direction.)

Finally, it is necessary to correct for small variations in sound velocity with changing composition, such as humidity variations.

The system described by [Lassila and Korpelainen, 2003] addresses many of the concerns raised above. A schematic picture is shown in Figure 4.3.2. The system implements a bi-directional acoustic measurement with the acoustic path arranged symmetrically relative to the optical path (effectively no Abbe offset) so as to ensure a fixed relationship between the acoustic and the optical pathlengths. A cross-correlator determines the precise delay of the received pulse relative to the pulse sent out. Good performance of the acoustic technique over a $5 \mathrm{~m}$ path in the laboratory has been demonstrated: refractive index variations induced by an electric heater are reduced from

about $1 \mu \mathrm{m}$ to about $0.2 \mu \mathrm{m}$ by using the acoustic compensation technique. However, the ultimate usefulness of the device cannot be fully evaluated until it is tested in a complex environment such as a construction site. 


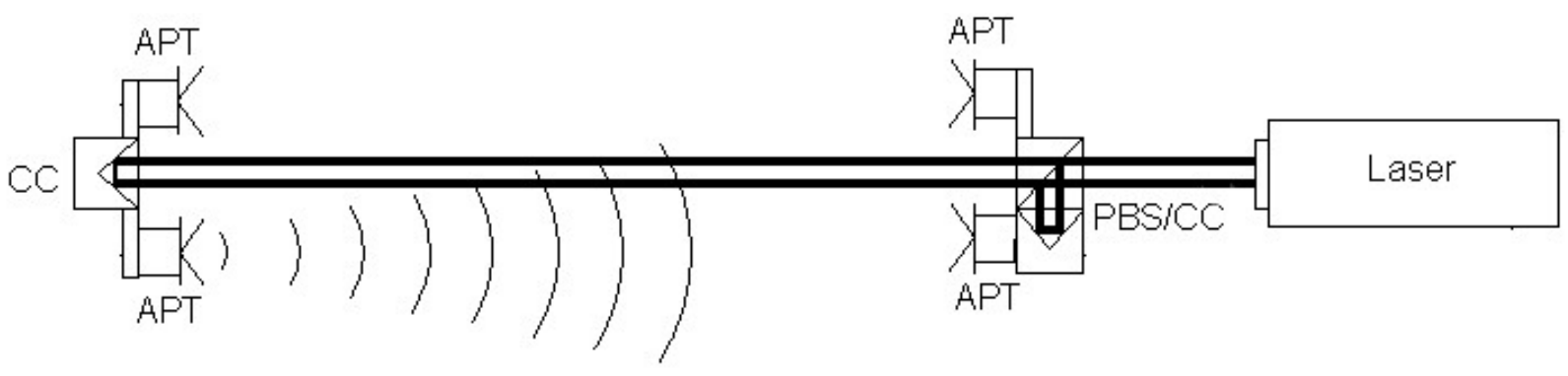

Figure 4.3.2: Schematic diagram of an acoustic system for measurement of air temperature. The picture is adopted from Figure 1 of [Lassila and Korpelainen, 2003]. The acoustic transducers (APT) are symmetric about the interferometer light path, which runs between the polarizing beamsplitter and reference corner cube (PBS/CC) and the measurement-path corner cube (CC).

\subsection{Angular Errors: Magnitude of the Problem}

Refractive index gradients bend light, giving rise to angular errors. As stated previously, we may distinguish two types of errors: errors on a short time scale reflecting rapid, turbulent variations in the refractive index, and slowly-varying errors reflecting persistent gradients in the refractive index that cannot be removed effectively by time-averaging. Short-term angular variations on a 1 second time scale when measuring over a 50-m path are $2 \mu \mathrm{rad}$ to $8 \mu \mathrm{rad}$ [Husler, 1989]. Flach and Mass [1999] report readingto-reading variations of about $0.4 \mathrm{~mm}$ measuring across a $86 \mathrm{~m}$ grass-covered field, corresponding to $5 \mu \mathrm{rad}\left(0.0003^{\circ}\right)$ angular fluctuations.

Persistent errors over larger spatial scales might be expected to cause more significant problems, as shown below.

Potential errors from angular refraction by temperature gradients may be estimated as follows. Consider a temperature gradient $d T / d h$ where $h$ is perpendicular to the direction of light propagation. (Most often, the gradient will be in the vertical direction, and $h$ will designate height.) As explained in detail in Figure 4.4.1 below, when a light beam travels a distance $d z$ perpendicular to this direction, then the angular deviation $\Delta \theta$, in radians, can be easily estimated by considering the change in wavefront direction as the portion of the beam traveling through the high-index (n), low-temperature gas lags behind the portion traveling through the region with lower refractive index and higher velocity:

$$
\Delta \grave{\mathrm{e}}=\left(\frac{d n}{d h}\right) d z \approx\left(\frac{d T}{d h}\right) \times 10^{-6} d z
$$




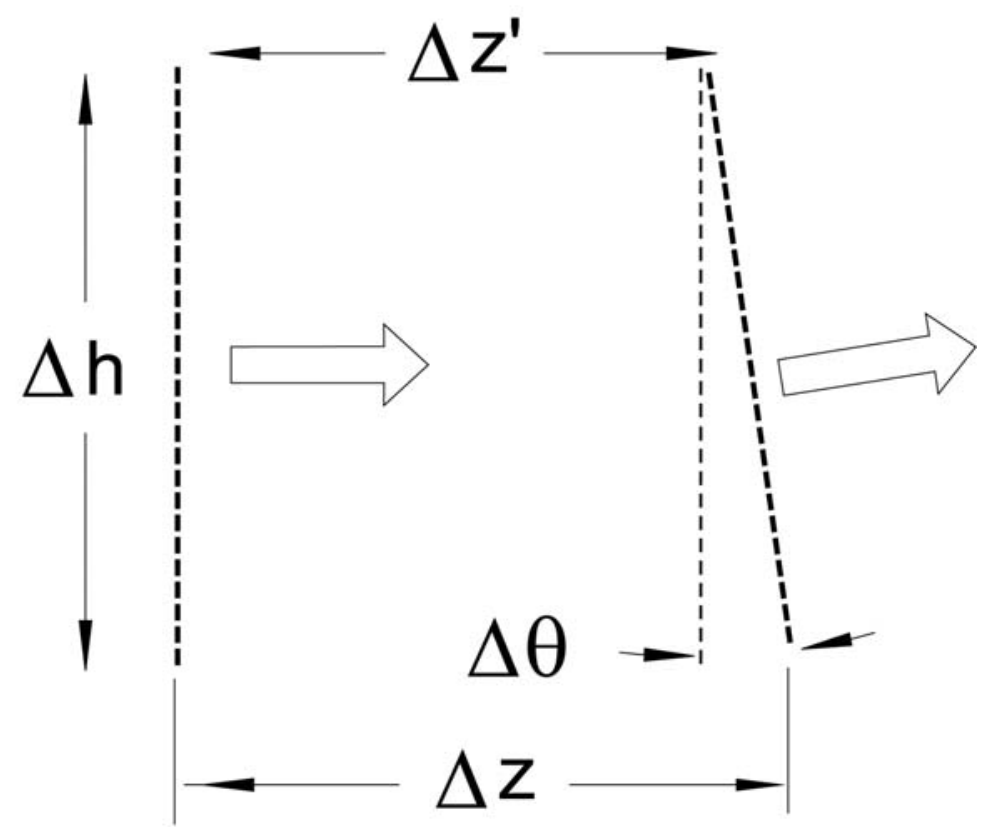

Figure 4.4.1 Bending of a beam by a refractive index gradient. The wavefront (heavy dotted line), traveling from left to right over a distance $\Delta z$, is bent by an angle $\Delta \theta$ as a consequence of the difference in refractive index along the two paths. If the refractive index along the top is greater than the refractive index along the bottom by $\Delta n=\Delta h \times(\mathrm{d} n / \mathrm{d} h)$, then the wavefront at the top lags the wavefront at the bottom as a consequence of the lower light velocity in regions of higher refractive index: $z$ ' differs from $z$ at the bottom by an amount $\Delta z \times(\Delta n / n) \sim \Delta z \times \Delta n$. Thus $\Delta \theta=(\Delta z \times \Delta n) / \Delta h$, from which equation 4.1 follows immediately. In this first approximation the angular deviation is a linear function of the travel distance $z$, and in a uniform gradient the cumulative perpendicular displacement of a beam over the pathlength $z$ is $z^{2} x(\Delta n / \Delta h) / 2$.

Eq. 4.4.1 assumes that $n \sim 1$, that $\Delta \theta$ is small, and that the refractive index varies by about $1 \times 10^{-6}$ per degree Celsius. The actual error in an angular observation depends on where along the measurement path the deviation $\Delta \theta$ occurs, because the lateral deflection of the beam depends on $\Delta \theta$ and on the distance to the target. The angular error will be equal to $\Delta \theta$ for a deviation at the location of the measurement instrument but will be zero if the deviation $\Delta \theta$ occurs right at the target. Taking this factor into account, and within the context of the approximations given above, the overall measurement error $\alpha$ can thus be obtained from the integral:

$\alpha=(1 / L) \int_{0}^{L}(z-L)(d n / d h) d z=10^{-6}(1 / L) \int_{0}^{L}(z-L)(d T / d h) d z \quad$ Eq. 4.4.2

where $L$ is the path length and the gradient $d T / d h$ is a function of position $z$ along the path. It may be noted that in this case where a uniform gradient extends over the 
entire measurement path, the angular error is effectively what would occur if the measurement system had an encoder error $\Delta \theta$ of $\operatorname{zx}(\Delta \mathrm{n} / \Delta \mathrm{h}) / 2$, or half the value given in equation 4.4.1.

The simple formula 4.4 .1 is useful for estimating the general magnitude of thermal-gradient induced beam bending. The more complex problem of beam propagation through turbulence will be discussed in Section 4.6. Examples based on the simple formula are given below.

A "typical" value for $d T / d h$ in geodetic measurements, at normal measurement heights of about $1.5 \mathrm{~m}$ above the ground, has been estimated by various authors as something between $0.25^{\circ} \mathrm{C} / \mathrm{m}$ [Flach, 2000] and $1^{\circ} \mathrm{C} / \mathrm{m}$ [Böckem, 2000]. Rüger [1996] gives a summary of various studies that measure light bending observed during geodetic measurements, from which thermal gradients can be inferred. This data shows that the magnitude of thermal gradients is a strong function of the distance above the ground, time-of-day, and cloudiness. On clear days, for heights of $1.5 \mathrm{~m}$ to $2 \mathrm{~m}$ above either grass-covered ground or ice, typically the gradient varies diurnally between about -0.3 ${ }^{\circ} \mathrm{C} / \mathrm{m}$ and $+0.5^{\circ} \mathrm{C} / \mathrm{m}$. At a height of $0.5 \mathrm{~m}$, diurnal variations from about $-1.7^{\circ} \mathrm{C} / \mathrm{m}$ to $+2.5^{\circ} \mathrm{C} / \mathrm{m}$ have been observed.

If we use a value of $1^{\circ} \mathrm{C} / \mathrm{m}$ for the gradient, over a distance of $20 \mathrm{~m}$ this would give an angular deviation $\Delta \theta=20 \mu \mathrm{rad}\left(0.0012^{\circ}\right)$. This is a small error by most standards, but its potential impact for high-precision applications cannot be ignored; note that even this very small angular deviation is much larger than the angular resolution of MetricVision $^{\mathrm{TM}}$ angular encoders as given in Appendix A. The actual error in lateral measurement at $20 \mathrm{~m}$ in the presence of a uniform gradient of $1^{\circ} \mathrm{C} / \mathrm{m}$ would be 0.2 $\mathrm{mm}$.

Thermal gradients tend to be greatest near localized heat sources. Close to sun-heated walls, gradients are often several times greater than the estimate given above. Similarly, there are likely to be substantial gradients when, for example, the measurement beam passes through a small opening in a wall.

Gradients in the air layer right above a concrete road have been measured to be on the order of $40^{\circ} \mathrm{C} / \mathrm{m}$ [Gustavsson, 2002]. One might expect even greater gradients right above a black roof. A gradient of $40^{\circ} \mathrm{C} / \mathrm{m}$, over a distance of $10 \mathrm{~m}$, will deflect light by $400 \mu \mathrm{rad}\left(0.02^{\circ}\right)$. This is a very significant error but it also clearly represents an extreme case.

Localized heat sources can generate significant localized disturbances, as is apparent visually when looking through the exhaust of motorized equipment. Even much smaller heat sources can potentially produce non-negligible errors. We have observed gradients on the order of $5^{\circ} \mathrm{C} / \mathrm{m}$ to $10^{\circ} \mathrm{C} / \mathrm{m}$ in the vicinity of computer monitors or several 
feet behind the cooling fan of electrical instrumentation. These thermal plumes are on the order of $0.5 \mathrm{~m}$ wide and might give angular deflections as large as $5 \mu \mathrm{rad}$.

\subsection{Correcting angular measurements for refractive index variation}

As in the case of correcting range measurements, a straightforward but usually impractical approach for correcting angular measurements is to directly measure thermal gradients along the measurement path. Some more practical alternatives are available, but none is without drawbacks.

Some significant success in eliminating refractive effects has been achieved using models of turbulence and heat transfer [Böckem et al., 2000]. The basic concept behind this technique is the observation that persistent vertical thermal gradients in the atmosphere are driven by thermal processes which also give rise to turbulence, and the two can be related to each other using the Monin-Obukhov model or other semiempirical models. By measuring turbulence-induced scintillation (intensity fluctuations) or image dancing (variations in angle of arrival, giving rise to fluctuations in image position in the focal plane) it is possible to determine the parameters that characterize the turbulence (structure constant and inner scale) and with heat-transfer models this can be related to the thermal gradient along the observation path.

The method has shown promise although it may not be reliable under all atmospheric conditions and is particularly unlikely to achieve good results in complex non-uniform environments such as, for example, if half of the measurement path were over a parking lot and half over grass. For imaging LADAR systems, the most interesting implementation of this technique would be to determine turbulence parameters directly from measurements of fluctuations in image intensity and jitter in image position, without the need for additional equipment. This method has been applied to video theodolites [Böckem, 2000; Flach and Maas, 1999; Flach, 2000].

A relatively simple method has been demonstrated recently to compensate for fluctuating thermal gradients while measuring displacements perpendicular to the line of sight. The system [Shirley, 2001] is based on sending out two crossed beams of light with a small angle between them (or observing two point sources along two crossed lines of sight), and combining the two crossed rays with a small angle between them so as to form interference fringes at a detector. In the most advanced implementation of this method, the detector is located right next to the transmitting optics, and the two transmitted rays are reflected by a special optic attached to the structure whose motion is to be determined. (See Figure 4.5.1)

The position of the fringes changes in response to motions of the reflector perpendicular to the line of sight, but the position is insensitive to thermal gradients bending the rays. This insensitivity is achieved, for uniform thermal gradients along the measurement path, by employing a geometry where the rays cross at the center of the measurement path, so that the excess refractive index encountered by one beam in the first 
half of its travel is cancelled by an equal deficit in the second half. As demonstrated thus far, the system works only for uniform gradients but work is underway on methods of avoiding this limitation. It should be noted that, although the technique successfully eliminates the effects of changing thermal gradients, it cannot determine the actual gradient-free angle of observation without the help of additional non-optical equipment (such as levels) to determine angular relationships between the system components at the two ends of the measurement path. Furthermore, if this angular relationship cannot be held rigidly constant, it is necessary to measure and correct for any angular variations.

The most generally applicable method for eliminating gradient-induced angular errors

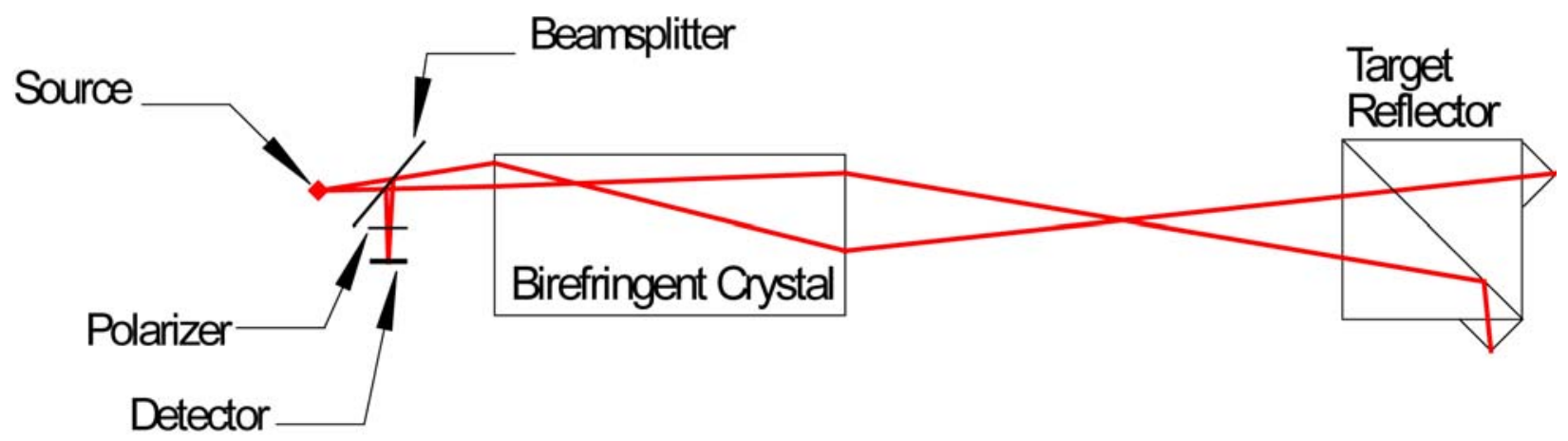

Figure 4.5.1 Compensation of angular errors using the method of Shirley. The birefringent crystal produces two polarization-encoded images of the source. The target reflector consists of a polarizing beamsplitter and two retroreflectors.

is a technique analogous to the two-color method previously described for correcting range data. Two-wavelength instruments for angle measurement ("dispersometers") have been discussed since the 1930's; an extensive review of early work is given by [Williams and Kahmen, 1984].

As a consequence of dispersion, bending is greater at shorter wavelengths, and the difference in bending of two beams of different color can be used to infer the absolute bending of both beams. As in the case of compensation of range data, the two-color angular compensation is very demanding because the difference in the bending of two beams is only a small percentage of the overall bending. Fashing and Hauser [1993] point out that, using their telescope with $1.65 \mathrm{~m}$ focal length and an objective of diameter $0.11 \mathrm{~m}$, the compensation of atmospheric bending at even a coarse level of $50 \mu \mathrm{rad}$ (10 arcseconds) requires measuring separations of $1.1 \mu \mathrm{m}$ between the two color spots, while the diffraction limit of their telescope is about 50X times larger than this value. Many instruments are capable of this performance at short distance, but for large-scale measurements the task is complicated by turbulence and scintillation. Although the measurement task seems daunting, Huiser and Gachter [1989] have apparently demonstrated microradian performance at a distance of $50 \mathrm{~m}$, using a theodolite that views a two-color light source (864 nm and $432 \mathrm{~nm})$. More precisely, 
they observe the variance in angle-of-arrival, and find that the standard deviation in the arrival angle is on the order of $1 \mu \mathrm{rad}$ for integration times of about $10 \mathrm{~s}$.

\subsection{Atmospheric Turbulence}

We have not yet discussed short-term fluctuations associated with atmospheric turbulence. The general problem of propagation of a beam of light through a turbulent medium, where the temperature distribution is given by a statistical distribution, can be treated using the Helmholtz scalar wave equation with the refractive index described statistically in terms of its power spectrum [Andrews and Phillips, 1998]. Calculations are usually based on a Kolmogorov-type model of turbulence. In the Kolmogorov picture, turbulence is modeled as a cascading series of eddies ranging in size from an outer scale (with a size that is of the order of half the height above the ground) to an inner scale below which eddies die out because of viscous dissipation. The inner scale is typically on the order of $5 \mathrm{~mm}$ at a height of $1.5 \mathrm{~m}$ above the ground. The larger eddies may refract a light beam ("beam wander") while smaller eddies give rise to scintillation, beam spreading, and loss of spatial coherence across a laser beam wavefront. For classical interferometric measurements these effects may cause the measurement signal to be lost entirely, while for imaging systems it will cause the image to dance across a focal plane detector. The details of these calculations, as developed by many researchers and summarized by Andrews and Phillips, are beyond the scope of this review. Below we list a few relevant formula from Andrews and Phillips that provide a starting point for estimating the magnitude of turbulence effects .

1) The strength of turbulence is measured by the index of refraction structure constant, $\mathrm{C}_{\mathrm{n}}{ }^{2}$. The structure constant can be determined by measuring instantaneous temperature gradients between nearby points in space or by using a scintillometer. Typical values are on the order of $10^{-13} \mathrm{~m}^{-2 / 3}$ at $1.5 \mathrm{~m}$ above the ground on a sunny day.

2)Turbulence can be characterized as "weak" or "strong" depending on the value of where $\mathrm{k}$ is the wavenumber of light and $\mathrm{L}$ is the range. Weak turbulence, corresponding to $\sigma_{1}^{2}<1$, leads to relatively simple analytic expressions for parameters that char-

$$
\sigma_{1}^{2}=1.23 C_{n}^{2} k^{7 / 6} L^{11 / 6}
$$

acterize the effect of turbulence on the beam. Assuming that we are using red light, with

and $L=100 \mathrm{~m}$, Eq 4.6.1 gives $\sigma_{1}{ }^{2}<0.17$, indicating that weak turbulence characterizes the applications of interest here. For a Gaussian beam with radius $W$ at the

$$
C_{n}^{2} \approx 2 \times 10^{-13} \mathrm{~m}^{-\frac{2}{3}}
$$


receiver, an additional requirement for weak turbulence is that where the Fresnel ratio, $\Lambda$, is given by

For a red beam of reasonable diameter at $100 \mathrm{~m}$ range $\Lambda$ is less than 1 and Eq 4.6.2 is not important.

$$
\begin{gathered}
\sigma_{1}^{2} \Lambda^{5 / 6}<1 \\
\Lambda=2 L /\left(k W^{2}\right)
\end{gathered}
$$

3) For weak turbulence, beam wander (random variations over time in the position of the beam centroid) and beam spread (an increase in short-timescale beam size) increase the effective size of the beam from a radius $W$ to an effective radius $W_{e}$ given by

$$
W_{e}=W\left(1+1.33 \sigma_{1}^{2} \Lambda^{5 / 6}\right)^{1 / 2}
$$

As an example, consider a Gaussian beam of red light that is collimated with $5 \mathrm{~mm}$ diameter, so that the radius at the source is $W o=2.5 \mathrm{~mm}$. At a range $\mathrm{L}=100 \mathrm{~m}$ the beam will spread due to diffraction, increasing the spot size from $W_{0}$ to

$W=W_{0}\left[1+\left\{2 L /\left(k W_{0}^{2}\right)\right\}^{2}\right]^{1 / 2}=8.4 \mathrm{~mm}$

Equation 4.6.4 now implies that turbulence will further increase the beam radius to $\mathrm{W}_{\mathrm{e}}$ $=8.8 \mathrm{~mm}$, a small increase relative to the diffraction effect. The turbulence-induced increase is primarily a consequence of beam wander with only a small contribution from short-timescale beam spread.

4) For a plane wave, variance in the angle of arrival, denoted $\sigma^{2} \beta$, is given by

$$
\sigma_{\beta}^{2}=2.914 C_{n}^{2} L D^{-1 / 3}
$$

where $D$ is the diameter of the receiver aperture. Equation 4.6 .5 is valid if $(L / k)^{1 / 2}<<D$. With $\mathrm{D}=25 \mathrm{~mm}$ and other parameters as given previously, this predicts that the stan- 
dard deviation $\sigma_{\beta}=14 \mu \mathrm{rad}$. More complex expressions predict fluctuations of the same order of magnitude for a Gaussian beam. Image dancing in the focal plane can be determined from $\sigma_{\beta}$ by multiplying by the focal length.

Additional measures of the effect of turbulence (including covariance functions, scintillation index, spatial coherence radius, short-term beam radius, etc.) can be calculated as well; the reader is referred to Chapters 6 and 7 of Andrews and Phillips (1998) for a complete discussion of weak turbulence. Additional atmospheric considerations such as spatial variations in the structure function and double-pass effects (enhanced backscatter) are also discussed by Andrews and Phillips. All of these atmospheric effects plus additional complications have been incorporated into complex LADAR models such as described by [Burton et al, 2002].

\subsection{Summary of refractive index effects}

In summary, non-uniformity of air temperature will cause refractive index variations that might, under unfavorable circumstances, give rise to errors as large as a few parts per million in range and possibly several hundredths of a degree in angle. Methods to eliminate these errors have been developed, but all of these techniques either have significant limitations or are very difficult to implement. Newer techniques show promise of overcoming many of the drawbacks of older methods but have not yet been tested under a variety of realistic conditions. Additional testing and development is needed to provide us with practical systems in which we have confidence. 


\subsection{Focal Plane Arrays}

In Chapter 2 we presented overviews of the primary technologies currently being used to achieve light-based range measurement in operational LADAR systems. All of those technologies can be implemented as stand-alone, "0-D" single point measurement devices. When so done, and equipped with a pan (yaw) / tilt (pitch) mechanism that moves the physical sensor, or with a beam deflection system (Chapter 3 ) that raster-scans the illumination beam across the scene of interest, we can create a "scanning" LADAR.

The intent of this chapter is to summarize the state-of-the art in focal plane array (FPA) detector technology as applied to LADAR. We devote an entire chapter to this subject because this is an area that is seeing heavy $R \& D$ funding at this time, largely driven by a demand for real-time, medium accuracy sensors for the military. Certain of the approaches presented here, however, do have potential for miniaturization and extension to low cost high-resolution sensors in the class that would be suitable for machine control and surveying.

Although mentioned briefly in Chapter 2 (Figures 2.2.4 and 2.3.2) a focal plane array is a 2D chip in which individually-addressable photo sensitive "pixels" can be accessed. Early FPA detectors were developed as, first, television imagers and later as passive IR (infrared), and later as FLIR (Forward Looking InfraRed) detectors, the latter largely for military purposes. For an in-depth discussion that represents the state-of-the-art through 1996 in passive FPA detectors, see (Crowe et al., 1996). CCD FPAs in the early 1960s made it possible to consider "on chip" processing electronics, making it possible to multiplex the signal from a large imaging array in real-time. These were not, however, ranging devices. Additional electronics must be added to an FPA -- in the form of timing circuitry -- in order to turn an FPA into a LADAR detector. The addition of such timing circuitry, and ancillary signal conditioning, analog and digital components, costs "real estate." This circuitry must fit behind each pixel in the array and usually causes the pixel size to be large relative to the pixel sizes in digital cameras, for instance. This tends to limit the physical size of the array. As an illustration of how much this "pixel logic" costs, current video camera chips - which are passive devices whose response is tuned to the optical wavelengths - can have an FPA with well in excess of $1,000 \times 1,000$ pixels (the Nikon D1X camera uses a 3,000 $\times 1,960$ pixel chip e.g.). Yet the best fielded operational LADAR FPA is presently a $32 \times 32$ array. Laboratory demonstration tests have been performed with $128 \times 128$ arrays, but this is still more than an order of magnitude more sparse than a common digital camera CCD chip. Despite these difficulties, there are advantages to moving towards FPA LADARs or, as some have labeled them, "3D video cameras" (since most of the effort is being directed at acquiring the LADAR frames in real-time). We will discuss in detail these advantages in the following sections.

An FPA LADAR can superficially look a lot like a scanning 0-D LADAR (see Figure 5.0.1), particularly if the array size is small and the system must be scanned to cover 


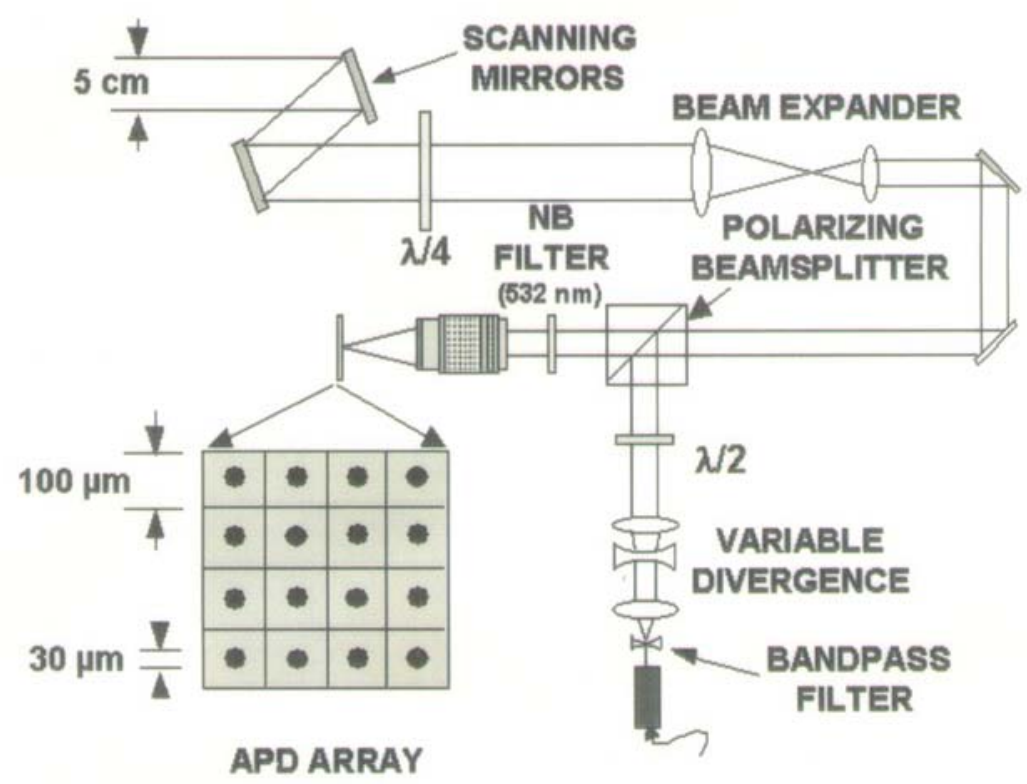

MICROCHIP LASER

Figure 5.0.1: Generic FPA LADAR architecture showing use of a $4 \times 4$ pixel APD (Avalanche PhotoDiode) detector. Courtesy Lincoln Labs

an overall field of regard that is larger than the sensor FOV. The distinguishing attribute of an FPA LADAR is that a significant number of independent range measurements are being made in parallel and, while not a requirement, a general design objective in such sensors is the achievement of real-time $(>10 \mathrm{~Hz})$ frame rates for the entire array. The process of sensor measurement parallelization is the crux of such designs. Implied in the notion of parallel sensor measurements is the necessity of each pixel in the array being illuminated by the return signal at the same time. Hence, there is a common lens that focuses the returned light onto the FPA. This has dramatic consequences on the power requirements of the illumination source (e.g. the laser). An " $\mathrm{x}$ $n$ " FPA represents an $n^{2}$ increased illumination power requirement, all other things held equal. The alternatives to increased illumination power include larger receiver apertures, photoelectron boosting techniques, and the use of single-photon detectors. In the sections below we present four distinct solutions that have been developed into operational LADAR systems and discuss their differences with respect to detector and ROIC design.

\section{$\underline{5.1 \text { All CMOS Solutions }}$}

Photonic Mixing Devices (PMDs) [see Section 2.2], can be fabricated using all-CMOS fabrication methods (as opposed to, e.g. hybrid approaches using InGaAs photodetectors and backing CMOS ROICs). In either case, each pixel must be accompanied by a readout circuit. The PMD approach is inherently one that can be integrated into a monolithic FPA chip and fabricated using standard CMOS processes. 


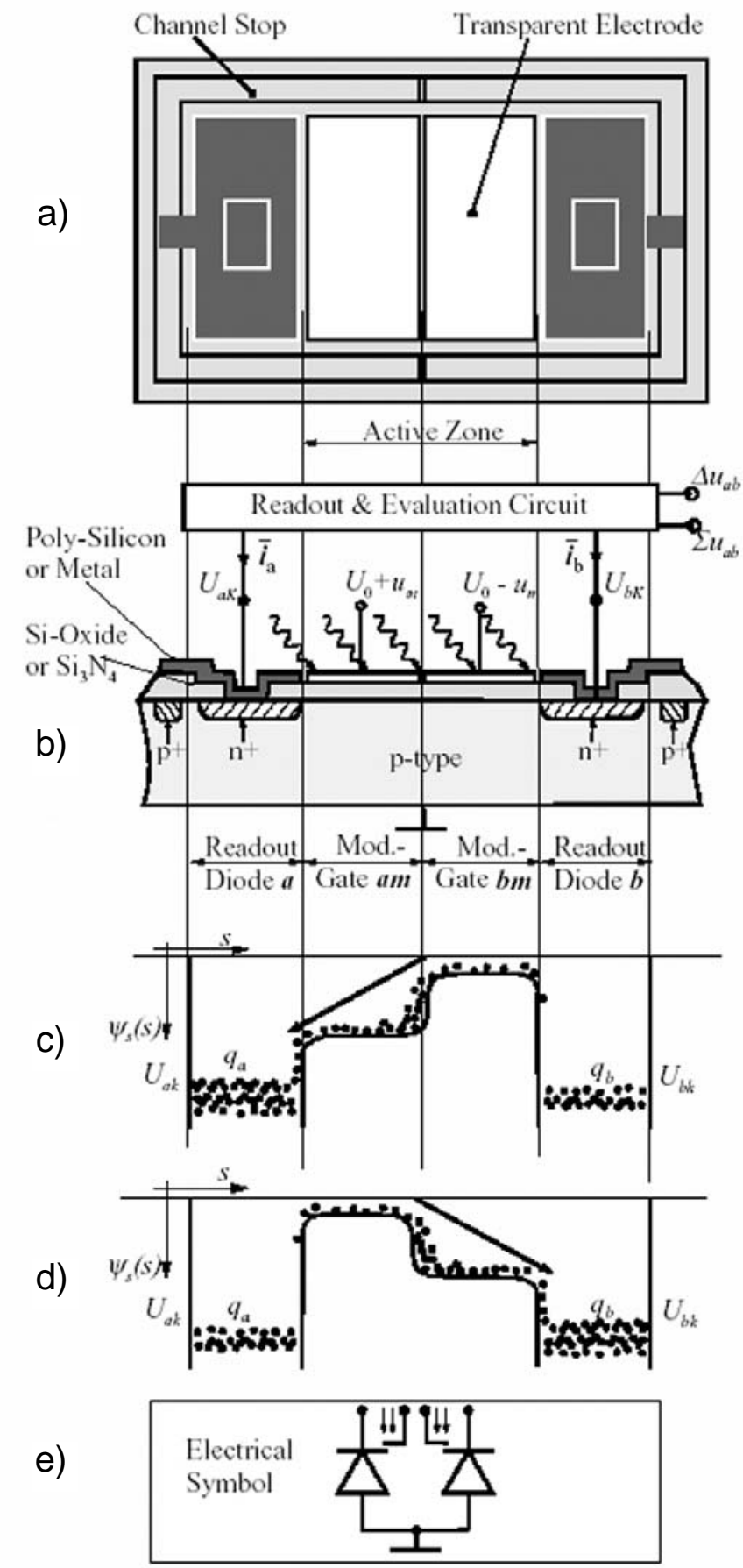

Figure 5.1.1 Photonic Mixing Concepts. The figure at left shows a simplified 2-gate PMD.

a) Overview of chip layout. The central areas are two transparent photo gates (the active, optical input window for receiving RFmodulated optical signals. The total optical active zone is square to maximize sensitivity.

b) Cross section of the layout showing readout circuit. The modulation gates 'am' and 'bm' with conductive and transparent electrodes, are isolated from the $\mathrm{p}^{-}$doped substrate by an $\mathrm{SiO}_{2}-$ or $\mathrm{Si}_{3} \mathrm{~N}_{4}$-isolation layer.

They are bounded between the left and right sides by $(n+)$-diffusion areas that form the cathodes of two pn-diodes 'a' and 'b'.

Positive voltages $U_{a K}=U_{b K}$ of about $5 \mathrm{v}$ are applied to reversely bias both diodes, with common anodes on ground potential.

c) \& d) illustrate the surface potential distributions for two different snapshot cases of $+/-u_{m}$, respectively

e) Electrical schematic of the 2-gate PMD representing a balanced optical mixer. The electrodes are associated with those described in b) above. The 2-gate PMD is a five terminal device with an optical input window. Two input electrodes, 'am' and 'bm', control the modulation gates that are connected to the push-pull RF-modulation voltage $u_{m}$, based upon a positive offset $U 0$ depending on the CMOS process. The maximum voltage $U_{0}+\left|u_{m}\right|$ should be lower than the cathode voltage $\mathrm{UaK}=\mathrm{U}_{\mathrm{bK}}$. The readout circuits process the photo currents ia and ib, delivering both the balanced mixing product as the output difference, $\Delta u a b$, and the total photo current as the output sum, $\Sigma \mathrm{u}_{\mathrm{ab}}$.

(Courtesy PMD Technologies $\mathrm{GmbH}$, Siegen, Germany) 
One approach to photonic mixing, taken by S-tec/PMD Technologies [Buxbaum and Gollewski, 2002* ] uses two transparent photogates as the active zone for receiving RF-modulated optical signals (see Figure 5.1.1). The modulation gates "am" and "bm" have conductive, transparent electrodes and are isolated from the ( $p-)$ doped substrate by an $\mathrm{SiO}_{2}$ or $\mathrm{Si}_{3} \mathrm{~N}_{4}$ layer. They are bounded on the left and right sides by $(\mathrm{n}+)$ diffusion areas that form the cathodes of two pn-diodes "a" and "b". Positive voltages $U_{a k}$ $=U_{\mathrm{bk}}$ of around $5 \mathrm{v}$ are applied to reverse bias both diodes.

The PMD operation principle is determined by the dynamic modulation process. The optical active area works at deep depletion mode. The potential distributions shown in Figure 5.1.1 c) and d) act like a "seesaw" that makes the carriers moving to the left and right in the potential wells. Thus, the mixing effect results from the synchronized charge drift and separation, which is caused by the applied push-pull RF-modulation voltages. Physically, the charge-transfer mechanism of the PMD is dominated mainly by the fringing field effect that governs the self-induced drift and the termal diffusion. PMD structure optimization means to enhance the dominant fringing field.

To understand the modulation process several characteristic cases should be discussed. If no push-pull RF-modulation voltage is applied, the photo-generated charges symmetrically drift to both cathodes ' $a$ ' and ' $b$ ' with a current difference of $\mathrm{i}^{\mathrm{a}} \mathrm{i}^{\mathrm{i}} \mathrm{b}=0$ or charge difference $q_{a}-q_{b}=D_{q}=0$.

As shown in Figure 5.1.1c) the surface potential configuration makes most of the photo generated electrons move always from the potential well under the right modulation gate $\mathrm{bm}$ to that under the left modulation gate am. Further, most of the carriers are coupled and drained to the readout diode a. However, some of the carriers drift and diffuse to the right readout diode $b$. For another case of surface potential distribution, as shown in Figure 5.1.1 d), most of the photo electrons are driven to the right potential well.

The averaged photo currents $\bar{i}_{a}$ and $\bar{i}_{b}$ can be sensed by an on -chip readout circuit in a way similar to a CCD camera, including reset MOSFETs. In practice a low impedance or current readout circuit is preferred beca use of eliminating feedback effects. The charge transfer of the device described here operates in the surface channel mode. It can also operate in a buried channel mode. The buried channel has a larger effective potential gradient under the electrodes $t$ hat speeds up the signal charges transferring to the readout diodes. Besides, the buried channel minimizes interface trapping, providing the charge transfer inefficiency as low as $10^{-5}$ at usual CCD -clock rates. Hence, the PMD is suitable to operate up to very high frequencies with high transfer efficiency in a short channel case.

\footnotetext{
* The majority of Section 5.1 is extracted directly from this document, which was commissioned by NIST under contract.
} 
Range calculation using a PMD approach is illustrated in Figure 5.1.2. If a constant flow of photons 'falls' into a homogeneously 'seesawed' potential well, the potential well separates the photo-generated charges into two equal parts, as shown in Figure 5.1.2 b. The device cannot determine the range in this condition. However, if the photons are timed or modulated, the potential well separates the photo-generated charges into one side; the charges are not equally collected as shown in Figure 5.1.2 c. For this extreme situation, one can determine the distance from the light source to the potential well by using the same modulation frequency for both the illumination source and as a bias on the potential well. For an ideal case of sinusoidal mixing with a mod-

$$
U_{a}=A\left[1+K \cos \left(\phi_{0}+\phi_{d}\right)\right]
$$

In which $\mathrm{A}$ is the local mean value, $\mathrm{K}$ the contrast, $\phi_{0}$ the phase offset, and $\phi_{d}$ the phase delay due to the time of flight. The output signal, $U_{b}$, at the readout port $b$, has a phase delay of $\pi$ due to the push -pull modulation voltage applied to the gates. Therefore:

$$
U_{b}=A\left[1+K \cos \left(\phi_{0}+\phi_{d}+\pi\right)\right]
$$

Evaluating several measurements (that is, measuring the voltage at 4 intervals $\pi / 2$ apart along one period of the modulated response) the phase delay $\phi_{d}=2 \pi f t_{d}$ can be obtained which corresponds to the time of flight. The $s$ ignal mixing (between the returned signal and the modulated voltage bias) takes place only in the PMD gate region. The fact that the mixing takes place on the CMOS chip itself means that a single-chip solution can be obtained, since it is possible using standard CMOS processes to also include the timing circuitry adjacent each pixel.

ulation frequency $f$, the mixed output signal $U_{a}$ at the readout port a can be expressed as:

\subsubsection{CMOS Readout Techniques}

The pixel pitch in an FPA designed using the PMD approach can be about the same size as those in a traditional CMOS sensing array. Therefore, the photo charge to be measured in a frame integration cycle is at a very low level. Like a CMOS sensor, it is usually not transported to an off-chip circuit without an on-chip readout circuit. The sensed signal is scaled by photo-generated charges in an area with a dimension of only several microns. The main task of the readout circuit is first to convert a small quantity of charge to a measurable voltage. Then the voltage is pre-amplified by an on-chip amplifier. The gain of the pre-amplifier is not crucial in comparison to its load ability and linearity for the PMD application case. With attention to detail the signal 

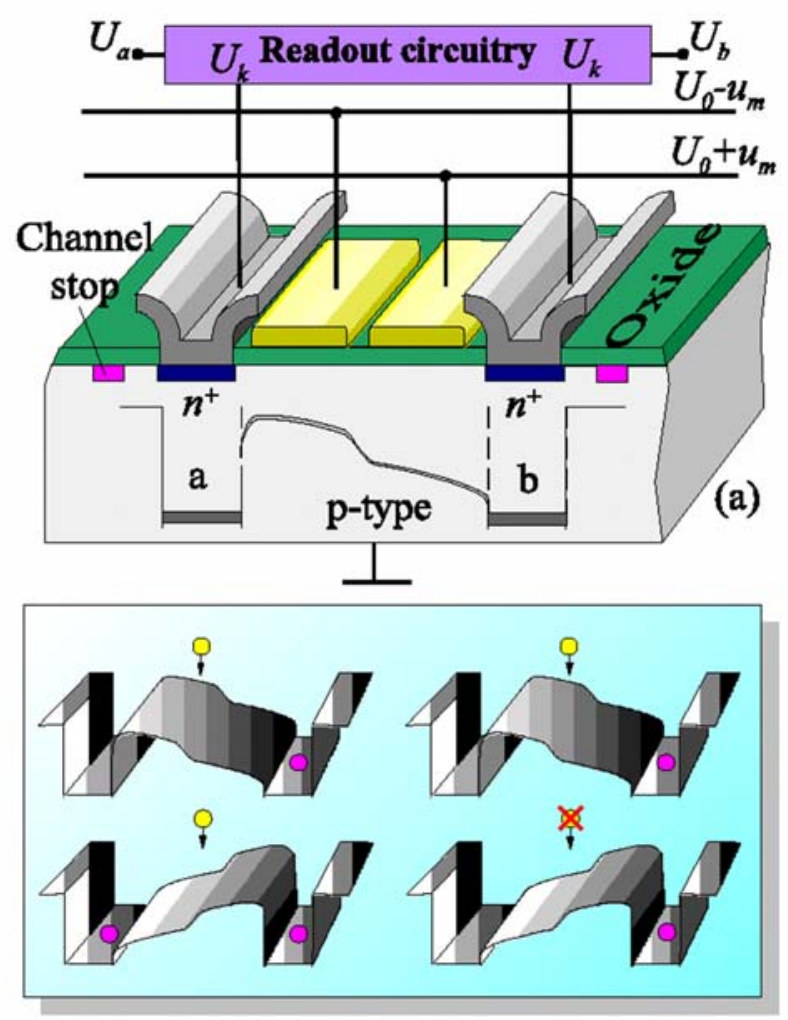

(b)

(c)

Figure 5.1.2 Simplified structure of a photonic mixing device. Figures $\mathrm{c}$ ) and d) illustrate the sensing and mixing processes of the PMD in two special cases. (Courtesy PMD Technologies GmbH, Siegen, Germany).

charge packet has less distortion so that it can be transported to an external location for advanced processing. The on-chip readout circuit performs charge to current conversion. Such a conversion can be achieved in a number of ways. Two that will be discussed below include floating diffusion sensing and current output sensing. It is possible to use CCD camera and CMOS camera technology to advantage in PMD design. The following discussion makes this clear.

\subsubsection{Floating Diffusion Readout}

When a signal charge is directly dumped to a small pre-charged floating diffusion capacitance on the chip, it causes a voltage variation across the capacitance. This method is called a floating diffusion sensing technique. An example of this approach is given in Figure 5.1.3, with a typical readout circuit serving as the structure for the floatThe diffusion capacitance $\mathrm{C}_{d}$ is first pre-charged by a reset MOS transistor M1. Then the diffusion capacitance $C_{d}$ remains in a floating state during the whole sensing process so that the signal charge $\Delta Q_{S}$ can be dumped to it. After a charge dumping cycle, a voltage variation $\Delta V_{s}$ at the capacitance is produced, which is approximately given by: 
ing diffusion.

$$
\Delta V_{S}=\frac{\Delta Q_{S}}{C_{d}+C_{G M 2}}
$$

$\mathrm{C}_{\mathrm{GM} 2}$ is the gate capacitance of the MOS transistor M2. The charge conversion efficiency (CCE) of the readout technique is reciprocally proportional to the sum of the $\mathrm{C}_{\mathrm{d}}$ and $\mathrm{C}_{\mathrm{GM} 2}$.

A very high CCE can be obtained by designing a small diffusion capacitance. However, the dynamic range or charge handling capability, on the other hand, is directly proportional to the sum. Therefore, a compromise should be made between CCE and the charge handling capability.

The floating diffusion technique can be easily fabricated by a standard CMOS process. It is the most frequently used readout technique. However, several facts should be taken into account if this technique is adapted to a PMD FPA. The floating diffusion capacitance is a voltage dependent variable. Therefore, the charge conversion causes

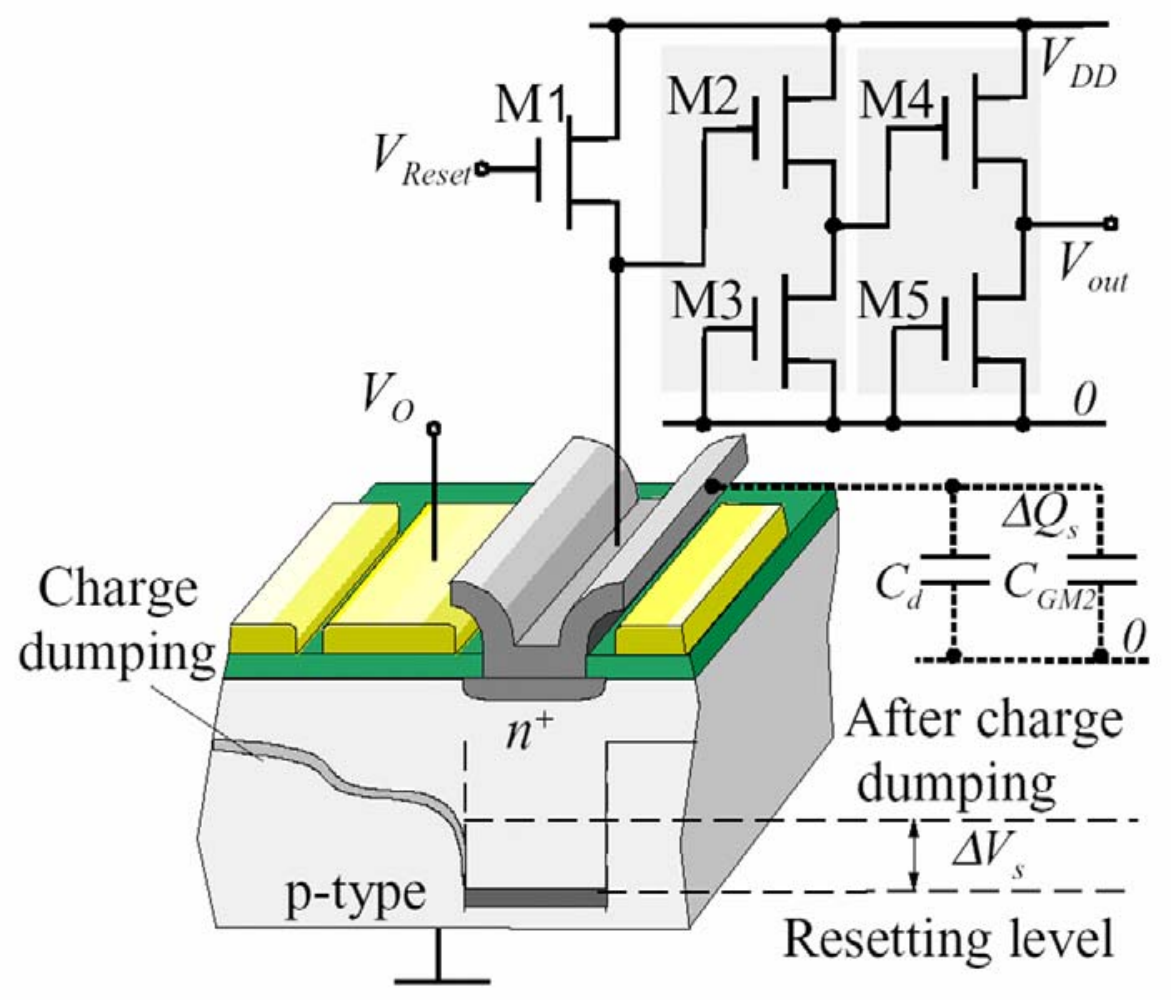

Figure 5.1.3 Floating diffusion sensing structure with associated readout for a PMD FPA pixel. (Courtesy PMD Technologies GmbH, Siegen, Germany). 
some non-linear distortion. In order to keep the floating diffusion less influenced by the side effect due to the modulation signal, an auxiliary electrode adjacent to the diffusion area is held at a constant voltage as a screen. Except for the MOS transistors M1 and M3, which remain as local on-chip circuitry, the transistors M4 and M5 can be treated as a sharing amplifier for a column or a row in a pixel array.

\subsubsection{Current Output Sensing Readout}

Another readout technique is to dump the signal charge on the diffusion capacitance that is fixed to a constant voltage value of $\mathrm{V}_{\text {ref }}$ by an amplifier as shown in Figure 5.1.4. The amplifier with capacitance feedback comprises a Miller integrator.

Therefore, the transported charge on the diffusion capacitance forces a current flowing in the feedback circuit, rather than accumulating in the diffusion region. The difference between this technique and the floating diffusion technique mainy lies in the readout circuits. Although the current will be ultimately be converted to a voltage, this technique is classified as a current-output approach. A voltage variation $\Delta \mathrm{V}_{\mathrm{S}}$ across the storage capacitance $\mathrm{C}_{\mathrm{S}}$ is given by:

$$
\Delta V_{S}=\frac{\Delta Q_{S}}{C_{S}+C_{P}}
$$

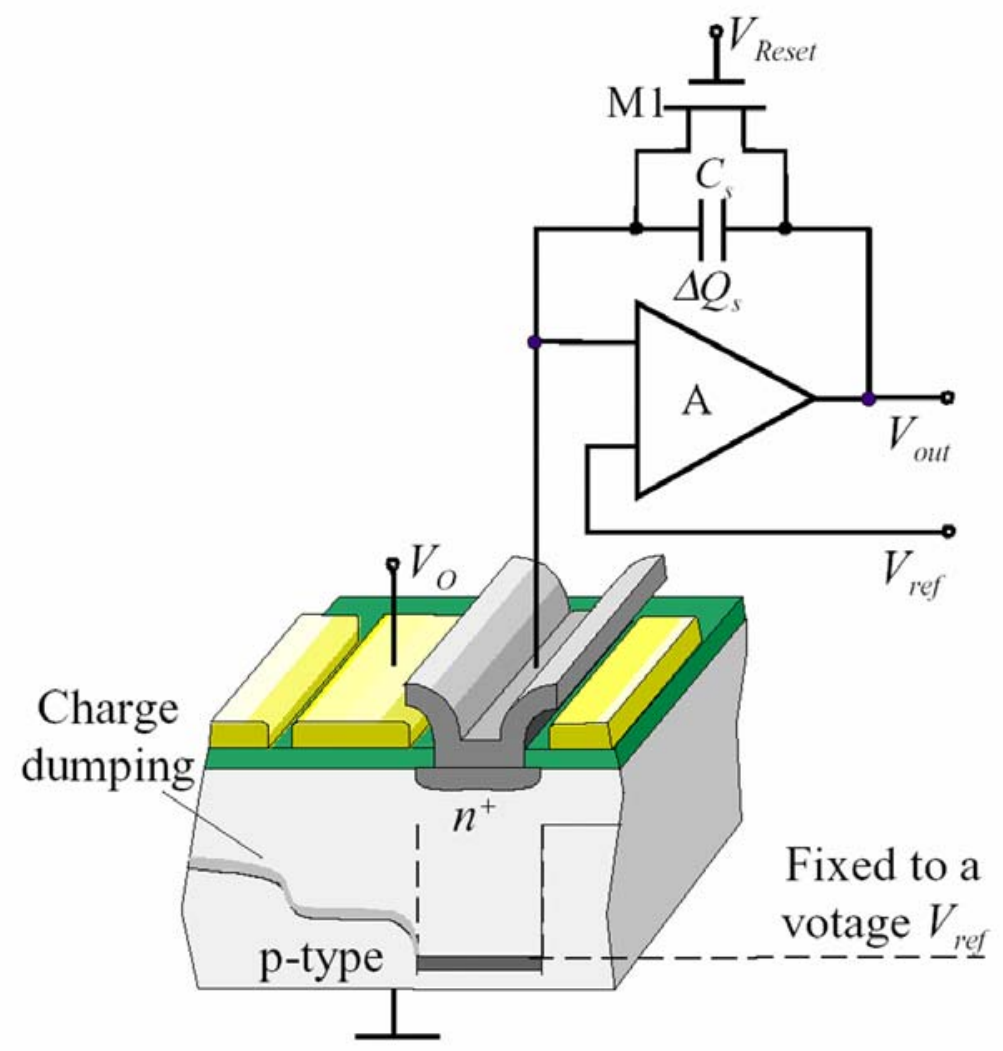

Figure 5.1.4 Current output sensing readout for a PMD FPA. pixel. Figure courtesy PMD Technologies $\mathrm{GmbH}$, Siegen, Germany. 
In the above equation the signal charge $\Delta \mathrm{Qs}$ is the integration of signal current over a time interval $\Delta \mathrm{T}$. Therefore the readout voltage CCE, as well as the dynamic range, is determined by the sum of $\mathrm{Cs}$ and parasitic capacitance $\mathrm{Cp}$, instead of the total capacitance of the diffusion region. In contrast to the floating diffusion technique, an additional noise source is involved in the readout circuit, i.e. the bias circuit for the constant voltage. This current output sensing method also brings advantages to the PMD application. Both readout diodes (i.e. the diffusion regions) are biased with the same voltage, therefore it eliminates the possible feedback of charge back-flow if the voltages across both diodes are unbalanced.

Moreover, there will be little or no signal charge accumulation in the diffusion regions. The charge handling problem is delivered to the storage capacitance Cs. this capacitance can be fabricated with two levels of poly-poly structure so that this circuitry has better linearity. The output gate will reduce the burden of the amplifier due to the RFinterference.

The approaches described above (see also Section 2.2) form the technical basis for several short range $(<10 \mathrm{~m})$, very compact, FPA LADARs that are now available commercially in small numbers from PMD Technologies GmbH and CSEM (see Appendix A). The accuracy of these devices is on the order of 5-10 mm and depends on both integration time and range. As explained in Section 2.2, these are phase-based, AMhomodyne devices and as such integrate whatever is within a pixel FOV to produce a range estimate. For angularly fully resolved targets, the accuracy will approach the numbers cited here; for non-resolved and multiple targets within a pixel FOV the results can stray dramatically. Currently, the illumination source for both commercial examples cited above is in the form of a modulated bank of normal IR-wavelength LEDs. This illumination power level presently limits the range of the sensor to under $10 \mathrm{~m}$. Furthermore, the design presented above is susceptible to saturation from stray DC illumination sources (e.g. bright sunlight) and background illumination suppression and/or compensation schemes must be implemented for outdoor daytime use.

As a further point of discussion, many proponents of FPA systems believe this to be the future of LADAR. One needs to take this with a grain of salt. A pure FPA LADAR, without any subsequent scanning of the FPA, may be sufficient for certain applications (e.g. military target recognition and homing). But it it likely to miss the mark entirely for general measurement applications, particularly engineering and construction applications. A case in point is shown in Figure 5.1.5. The upper image shows a $25 \times 64$ pixel FPA frame for a steel building beam equipped with a pre-fabricated connector, designed to enable robotic assembly. The Construction Metrology and Automation Group at NIST is developing methods for autonomous component identification, capture (e.g. using a specially modified crane), and placement. The picture at center left in Figure 5.1 .5 shows a digital image of the same scene. Even at close range it is evident that $25 \times 64$ pixels make for an extraordinarily coarse model -- one that would lead to a low probability of correct detection in the presence of hundreds of other similar components likely to be present in the lay down yard of the average construction site. 

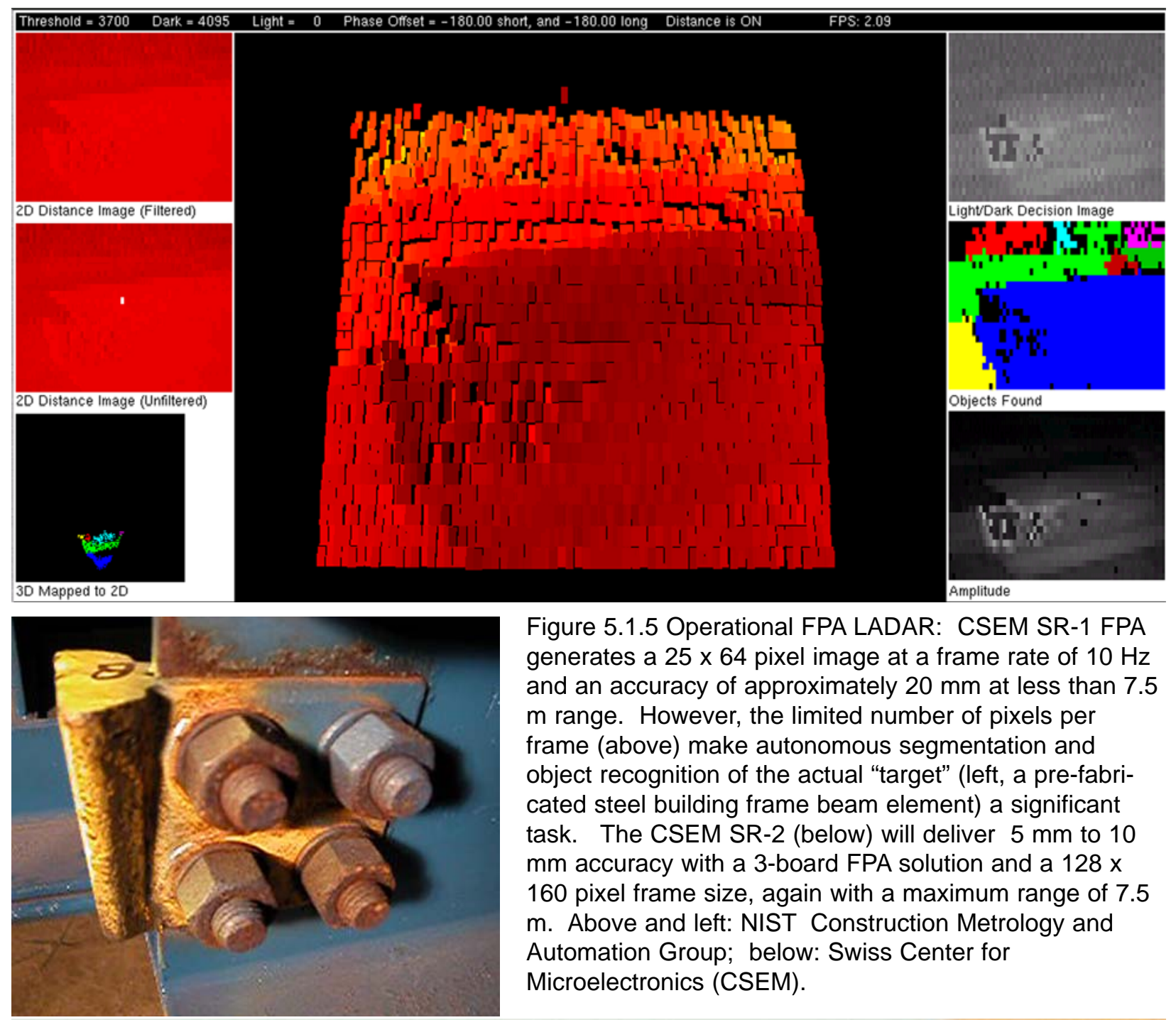

Figure 5.1.5 Operational FPA LADAR: CSEM SR-1 FPA generates a $25 \times 64$ pixel image at a frame rate of $10 \mathrm{~Hz}$ and an accuracy of approximately $20 \mathrm{~mm}$ at less than 7.5 $m$ range. However, the limited number of pixels per frame (above) make autonomous segmentation and object recognition of the actual "target" (left, a pre-fabricated steel building frame beam element) a significant task. The CSEM SR-2 (below) will deliver $5 \mathrm{~mm}$ to 10 $\mathrm{mm}$ accuracy with a 3-board FPA solution and a $128 \mathrm{x}$ 160 pixel frame size, again with a maximum range of 7.5 m. Above and left: NIST Construction Metrology and Automation Group; below: Swiss Center for Microelectronics (CSEM).

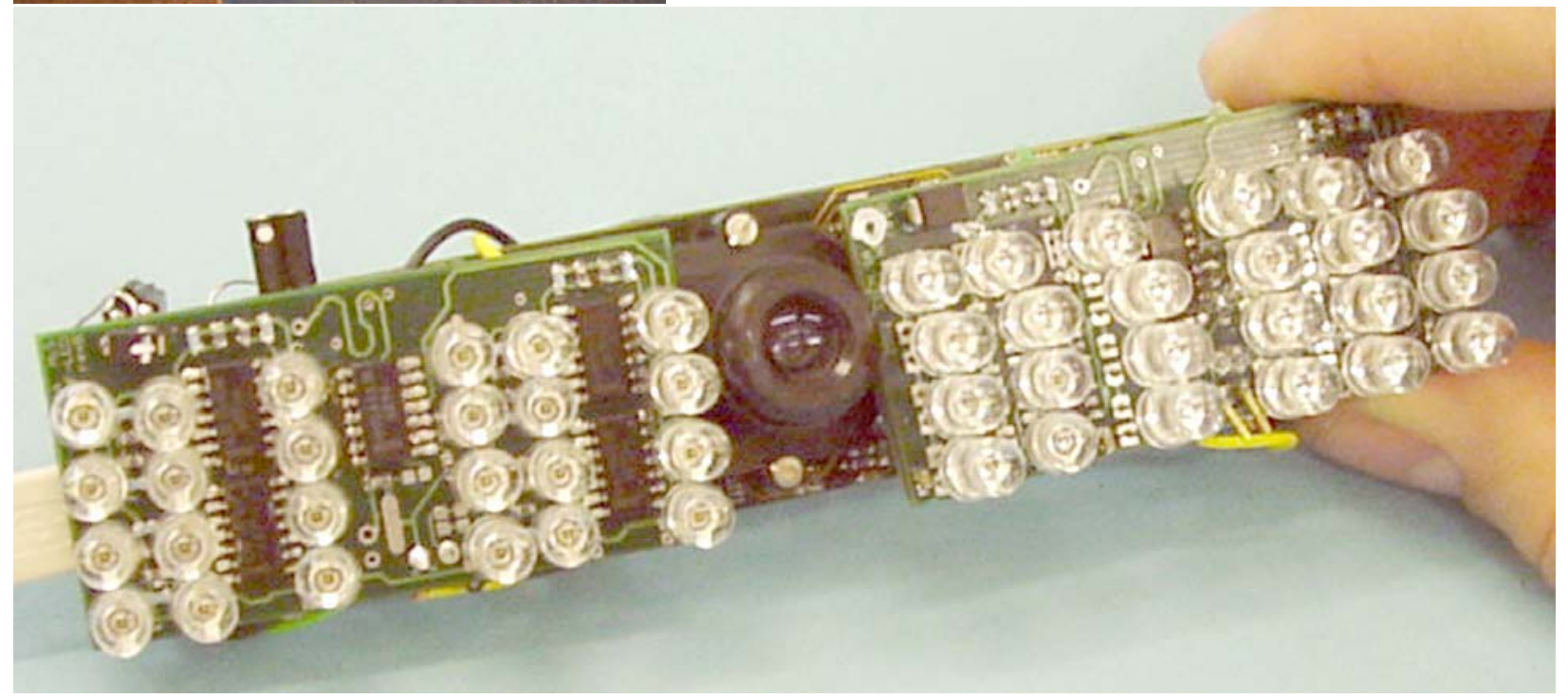




\section{$\underline{5.2 \text { Image Tube Solutions }}$}

The FPA design approach described in Section 5.1 can be considered a "homogeneous" approach in that the detector chip and ROIC are one and the same, fabricated on the same chip using the same CMOS processes. In general this is not the case. One particularly recurrent issue in LADAR design is that of eye safety. This is true even for the majority of military applications, since there can never be guarantees that friendly forces are not within the field of illumination. In scanning LADAR systems it is common to incorporate a "kill" feature that disconnects the laser power supply in the event that either of the scanning servos freezes or dies, such that the laser would be pointed at a single spot. This is an issue because most commercial scanning LADAR systems today operate in the $400 \mathrm{~nm}$ to $900 \mathrm{~nm}$ wavelength regime and eye safety requirements severly limit allowable laser power. This becomes of critical importance in FPA LADAR design because there the already limited photon count is spread over

the nxn array. There are two common solutions. The first is to switch detector chemistry. Figure 5.2.1 shows a few sample photonic materials and their spectral responsiveness to impinging light. Silicon, which forms the basis for the CMOS process described in Section 5.1 above, shows significantly reduced sensitivity (the ability to generate photo charge, or current per incident photon flux) beyond $1 \mu \mathrm{m}$ wavelength. Others, particularly InGaAs (Indium Gallium Arsenide) show peak performance in the $1.5 \mu \mathrm{m}$ to $1.6 \mu \mathrm{m}$ regime. Coincidentally, it is in this exact regime that the human eye can tolerate the highest light intensity without causing irrepairable damage. Other materials (e.g. HgCdTe, CdZnTe) exhibit similar behavior to InGaAs. For this reason, all are being considered presently for high power real-time FPA LADAR systems. But, these alternate chemistries are difficult to fabricate -- far more difficult and costly than silicon CMOS - and as a result high pixel density is limited. It is inefficient to attempt to place the ROIC (the timing read out integrated circuitry) on a non-Si detector chip. Thus, as shown in Figure 5.2.2, a hybrid sensor is required. The detector and ROIC are frequenty joined through the use of a "bump bonding" technique in which small "bumps" of either indium (In) or gold (Au) are used to fuse (solder) contacts between the two dissimilar fabrication components.

Yet another means for enhancing FPA detector sensitivity is through the use of an "imaging tube" as shown in Figure 5.2.3. An image tube is a device that was pioneered for night vision and infrared vision devices. Incoming photons (P in Figure 5.2.3) strike the high voltage photocathode plate that is located behind a transparent (at the wavelength of interest) window and inside an evacuated cavity. Electrons are generated from the back side of the photocathode. The electrons are then accelerated by the anode-cathode field ( $E$ in Figure 5.2.3) and cause impact ionization in the detector array. The higher impact energy leads to higher photo current output at each pixel of the FPA array. Because of this, it is possible to use a silicon-based (i.e. CMOS) detector (as opposed to the previously mentioned alternate detector chemistries). This approach allows the use of off-shelf silicon detector arrays. The ROIC, in the example shown in Figure 5.2.3, is provided by the aforementioned technique of bump-bonding to the back side of the detector chip. While this is a feasible 


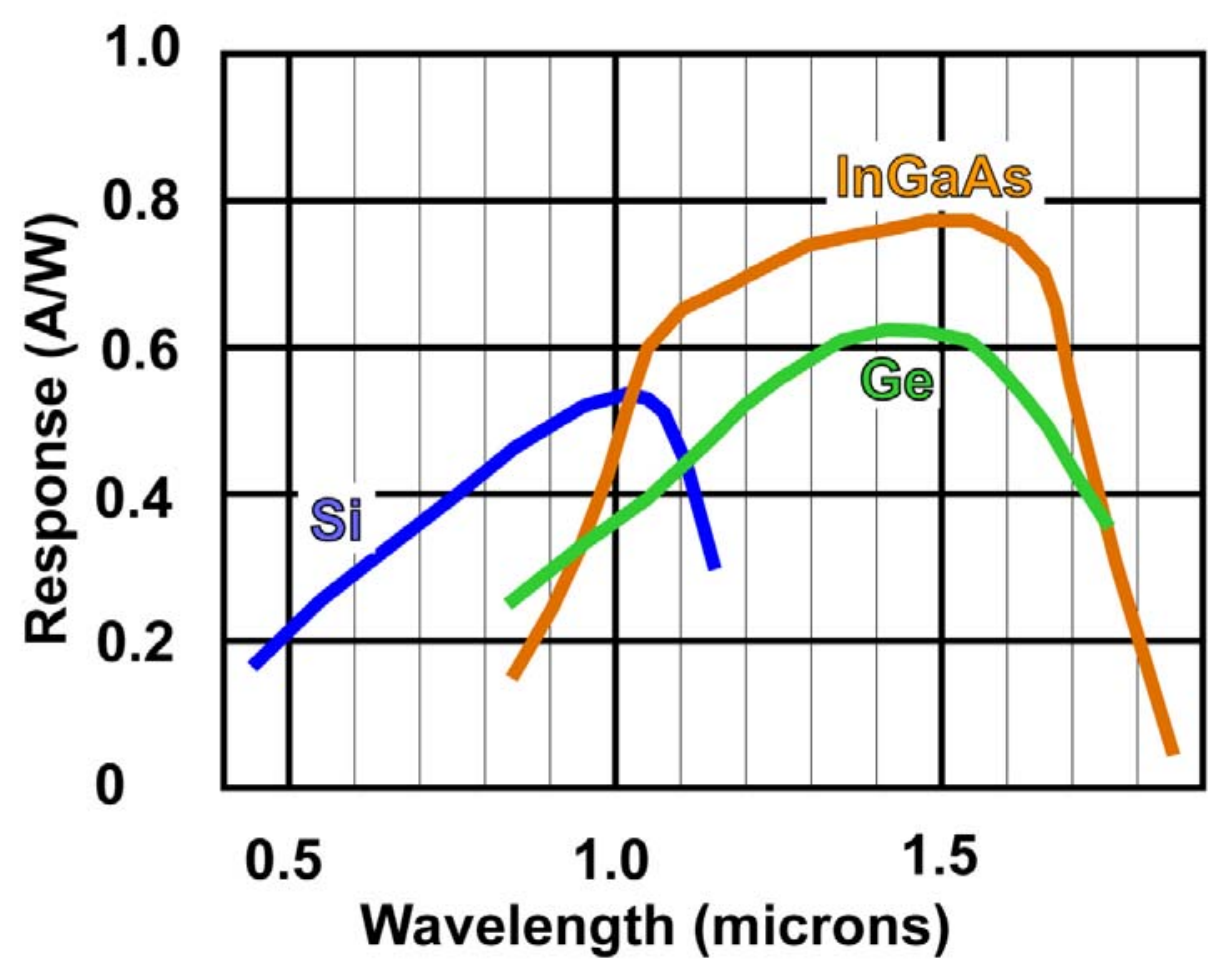

Figure 5.2.1 Spectral response for various detector chemistries.

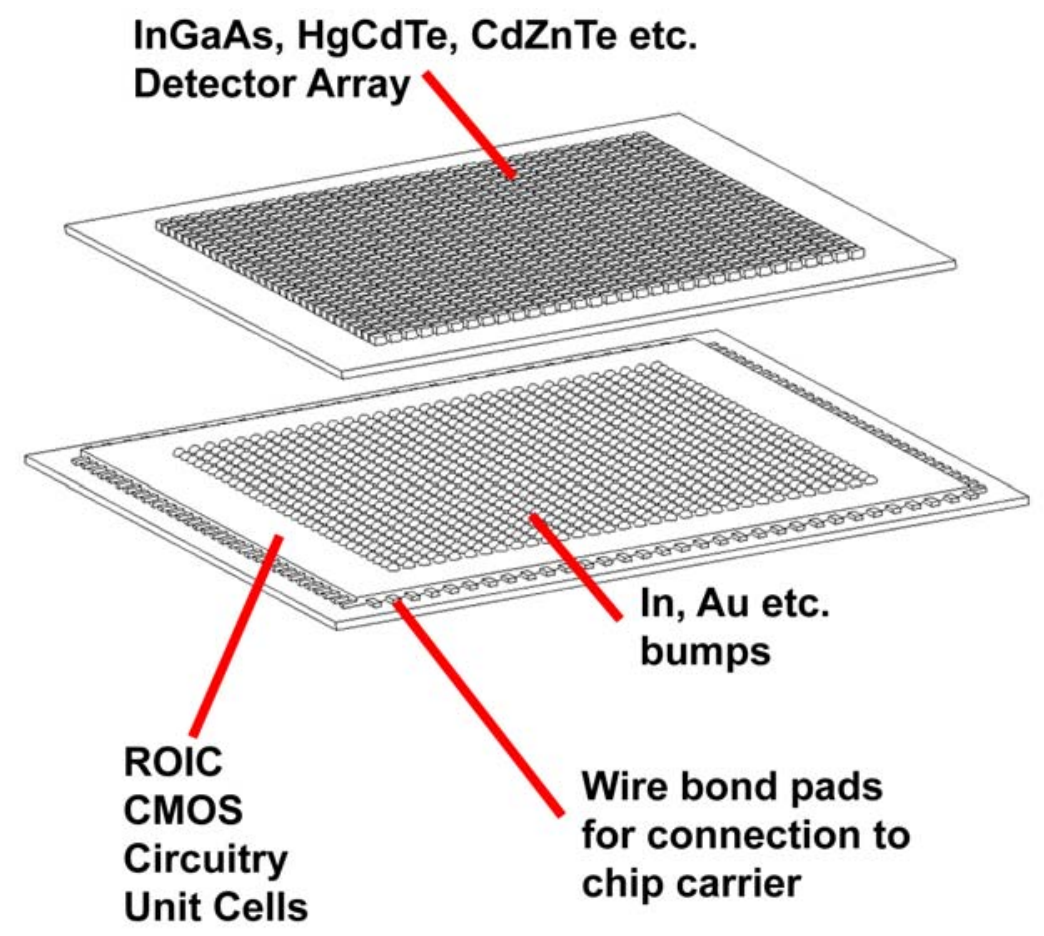

Figure 5.2.2 Traditional non-Si FPA detector and CMOS ROIC (read out integrated circuit) 

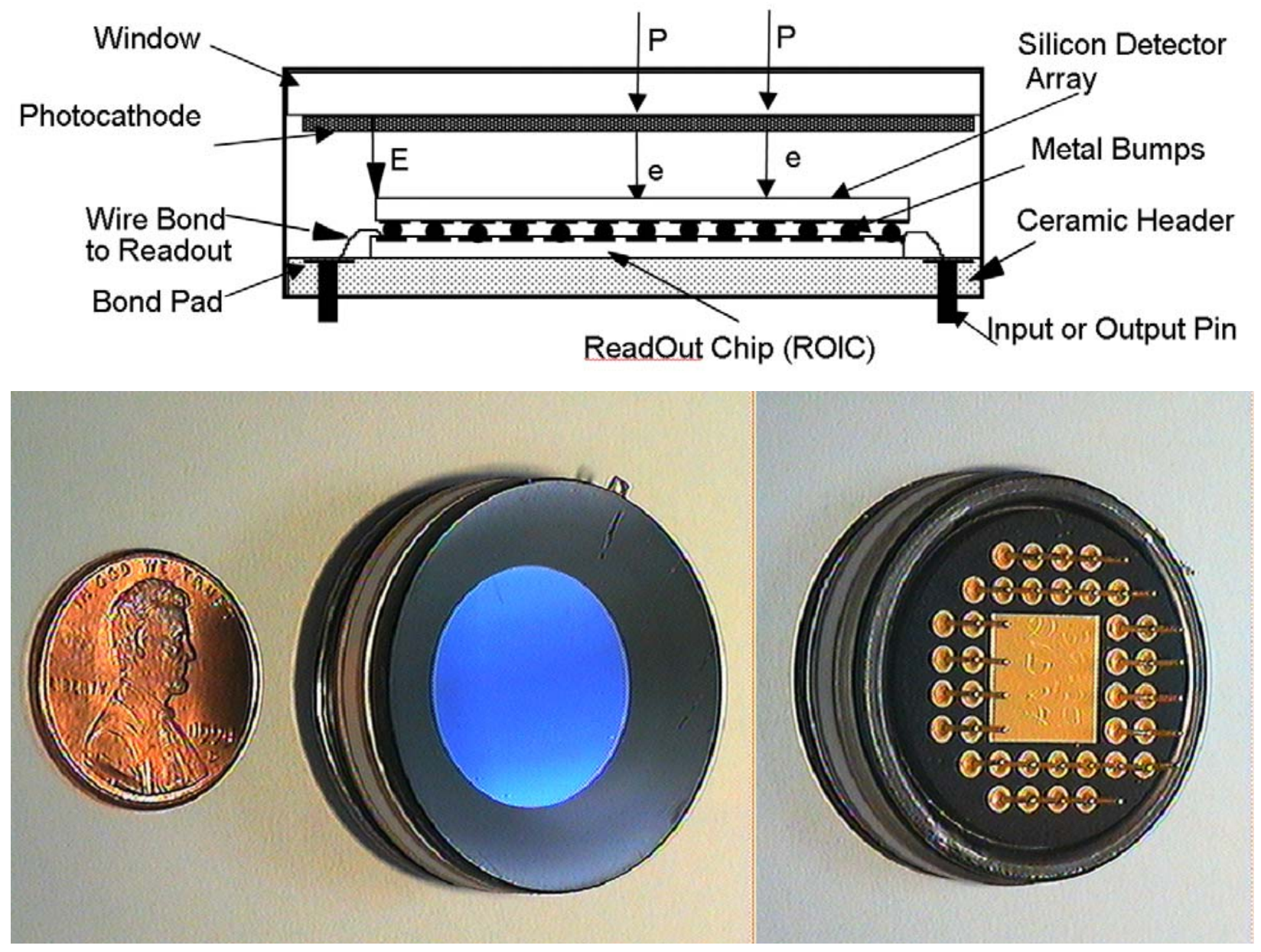

Figure 5.2.3 Image tube approach to increasing the sensitivity of an FPA. Upper schematic shows a cross section of the device; central images show front (left) and back of the integrated image tubedetector-ROIC sensor. Bottom figure shows a digital image of the $128 \times 128$ pixel detector array. (Courtesy Advanced Scientific Concepts).

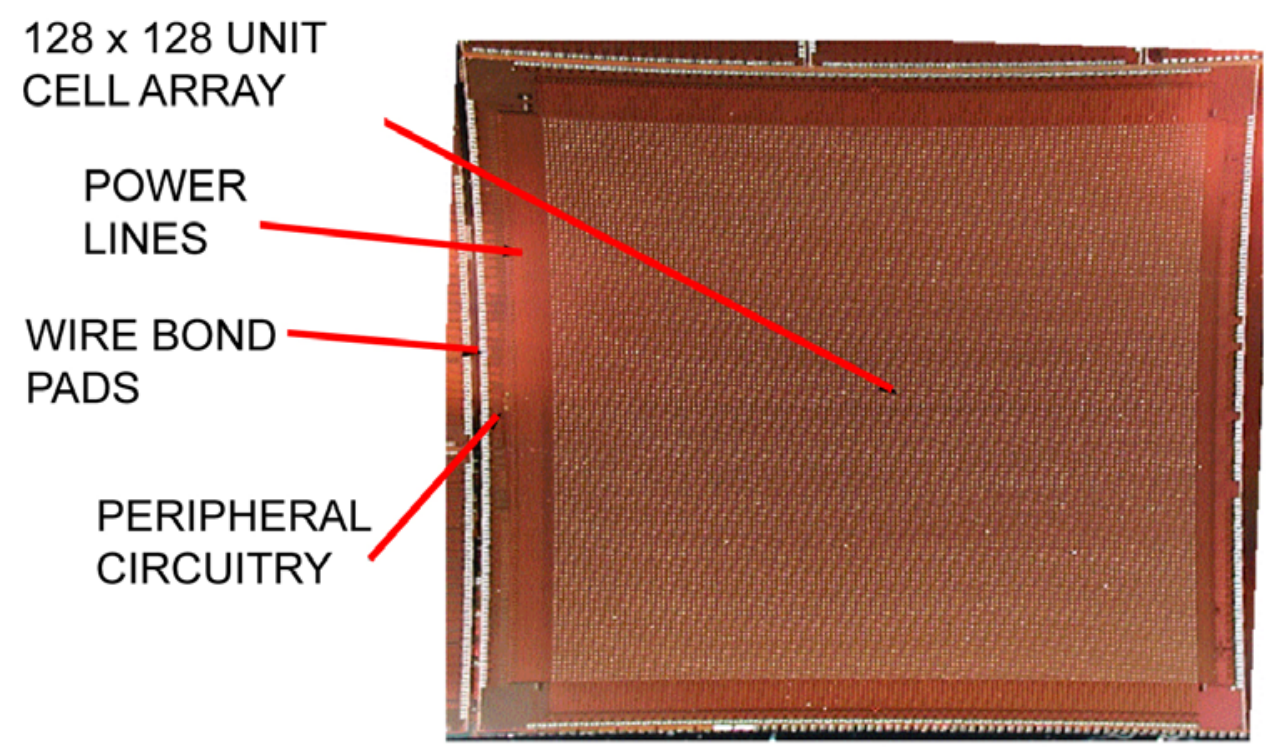




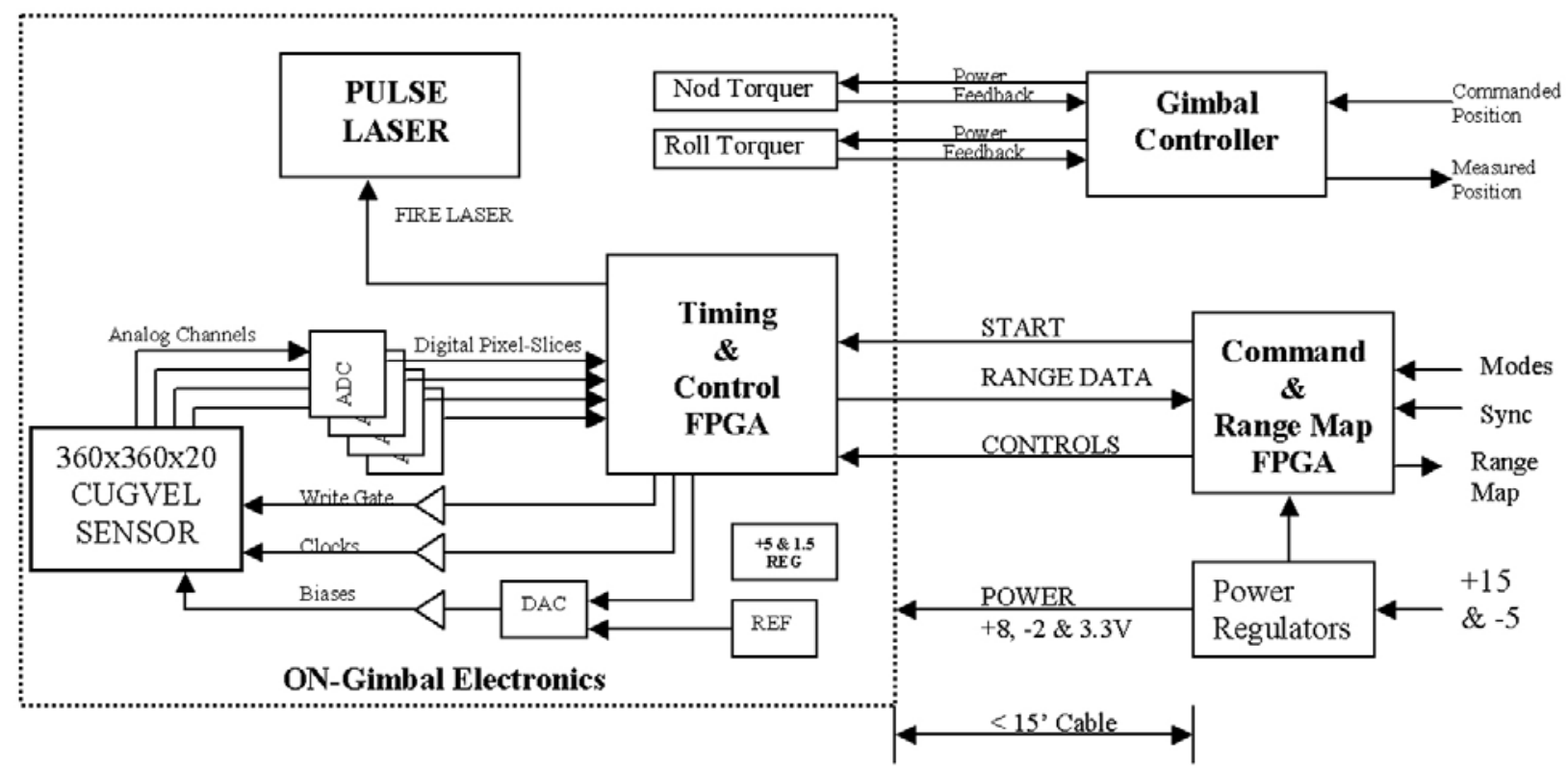

Figure 5.2.4 Typical FPA control architecure surrounding the sensor chip. (Courtesy Advanced Scientific Concepts).

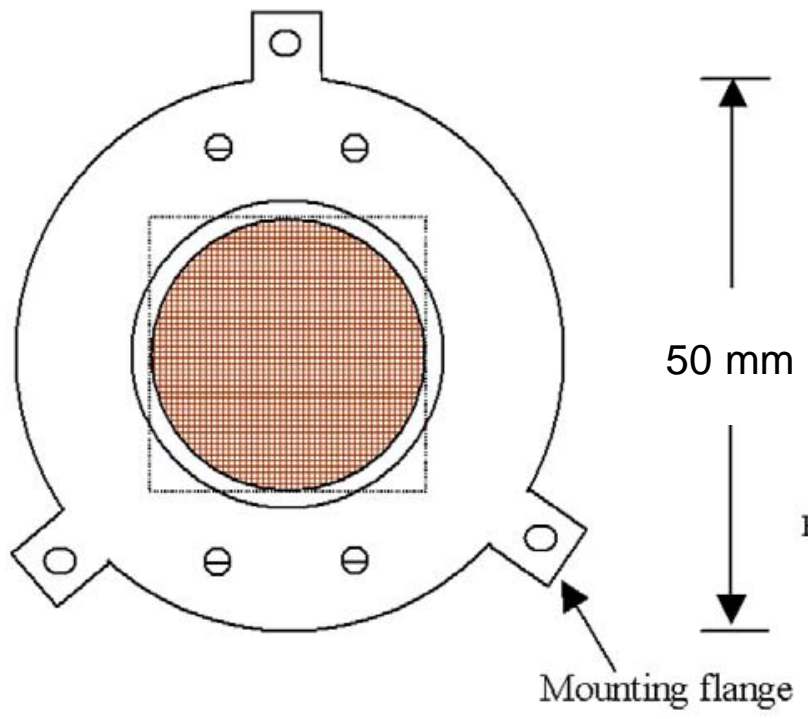

Front (Filter removed)
Light Out Sensor

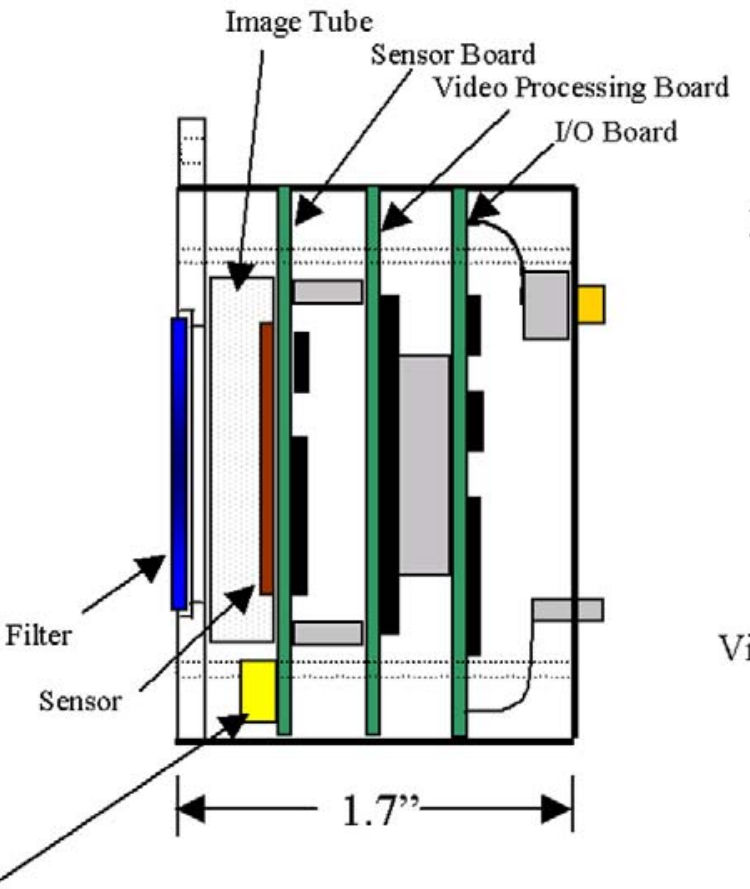

Side

(Cut-away)

Figure 5.2.5 Typical "brass board" FPA implementation.(Courtesy Advanced Scientific Concepts). 

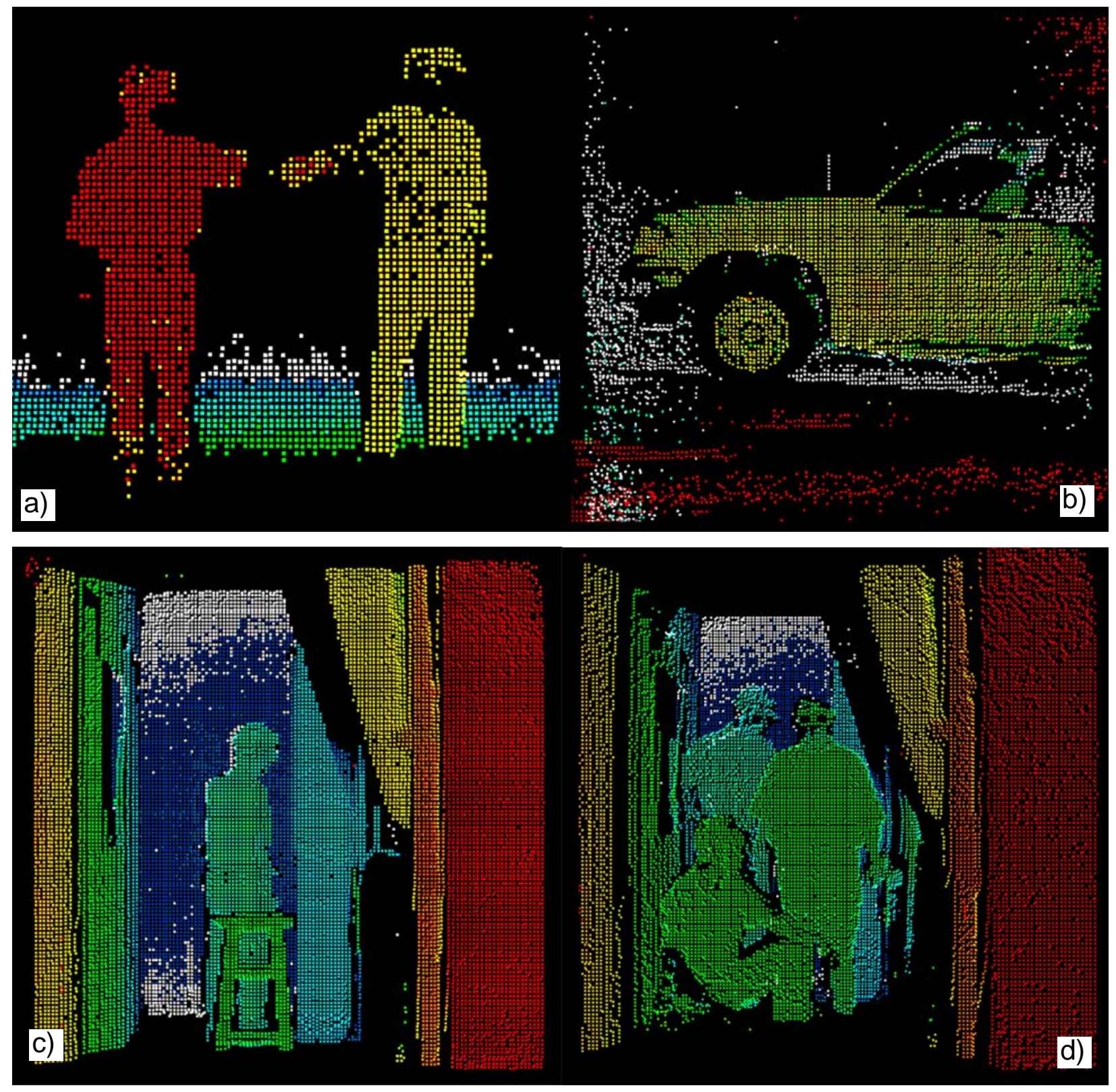

Figure 5.2.4 Four sample frames from a 128x128 pixel FPA. a) Upper Left: "shaking hands"; b) Upper Right: driver's side of passenger vehicle. Note the no-return zone in the area of the matte black tire; $c$ ) Lower Left: single individual seated on stool indoors; d) Lower Right: three individuals in various poses indoors. These figures in general continue the theme initially discussed in Figure 5.1.5. FOV is approximately $45^{\circ} \times 45^{\circ}$. For reference, compare Figures 5.1.5 and this set of figures with Figure 5.2.5. 


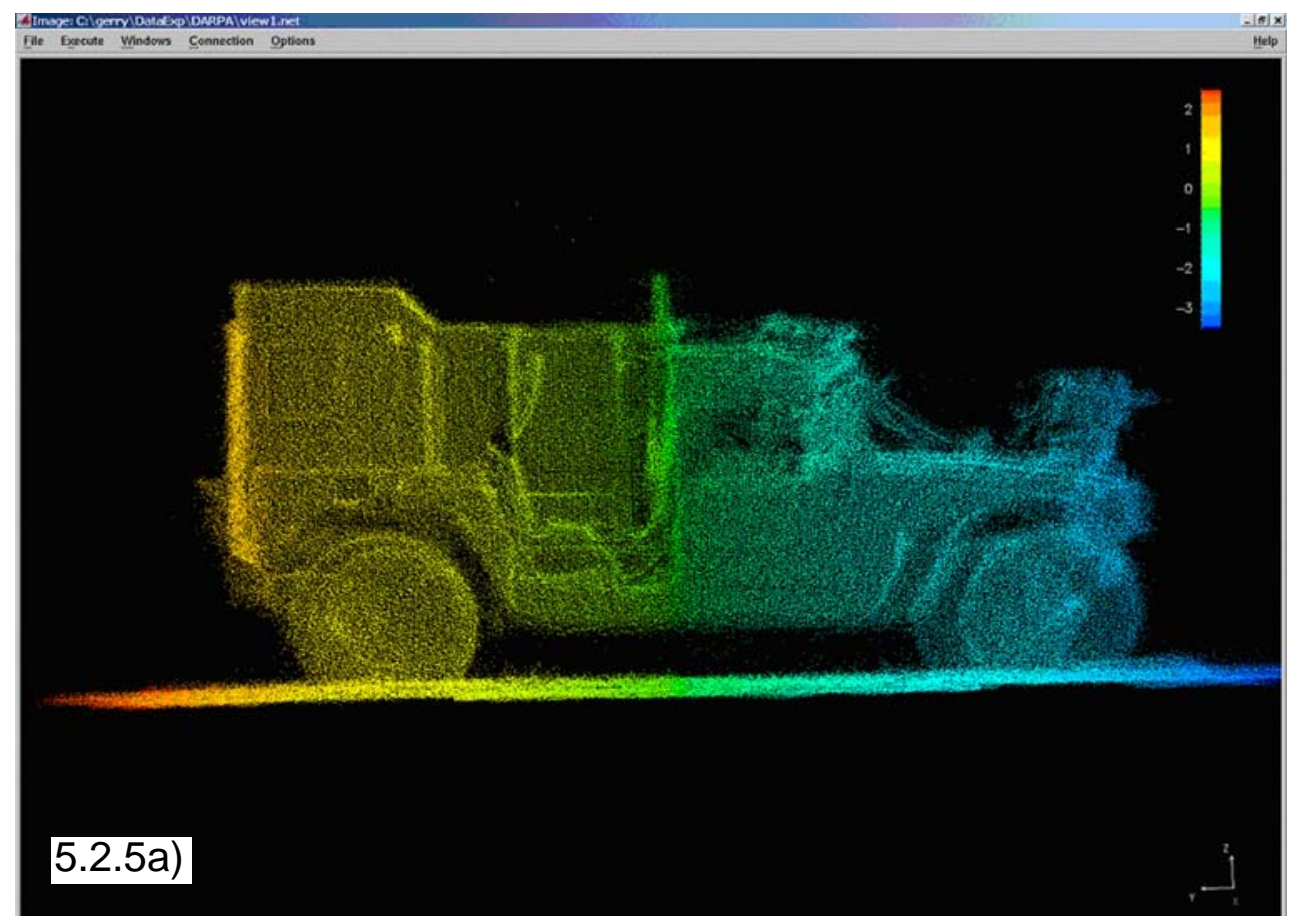

Figure 5.2.5: Typical large-scene images produced using commercial LADAR systems. FOV for the system that acquired the two images seen here (Humvee, top; Woods scene, below) is $80^{\circ} \times 330^{\circ}$. The angular resolution for these images is $0.072^{\circ}$-- at least three times finer than that shown in Figure 5.2.4, yet 36 times more coarse than some state-of-the-art commercial systems now available for survey work. In order to work at this high level of angular resolution a $128 \times 128$ FPA would have to have an optical FOV on the order of $0.25^{\circ} \times 0.25^{\circ}$ and would therefore have to be scanned in order to image any significant area.

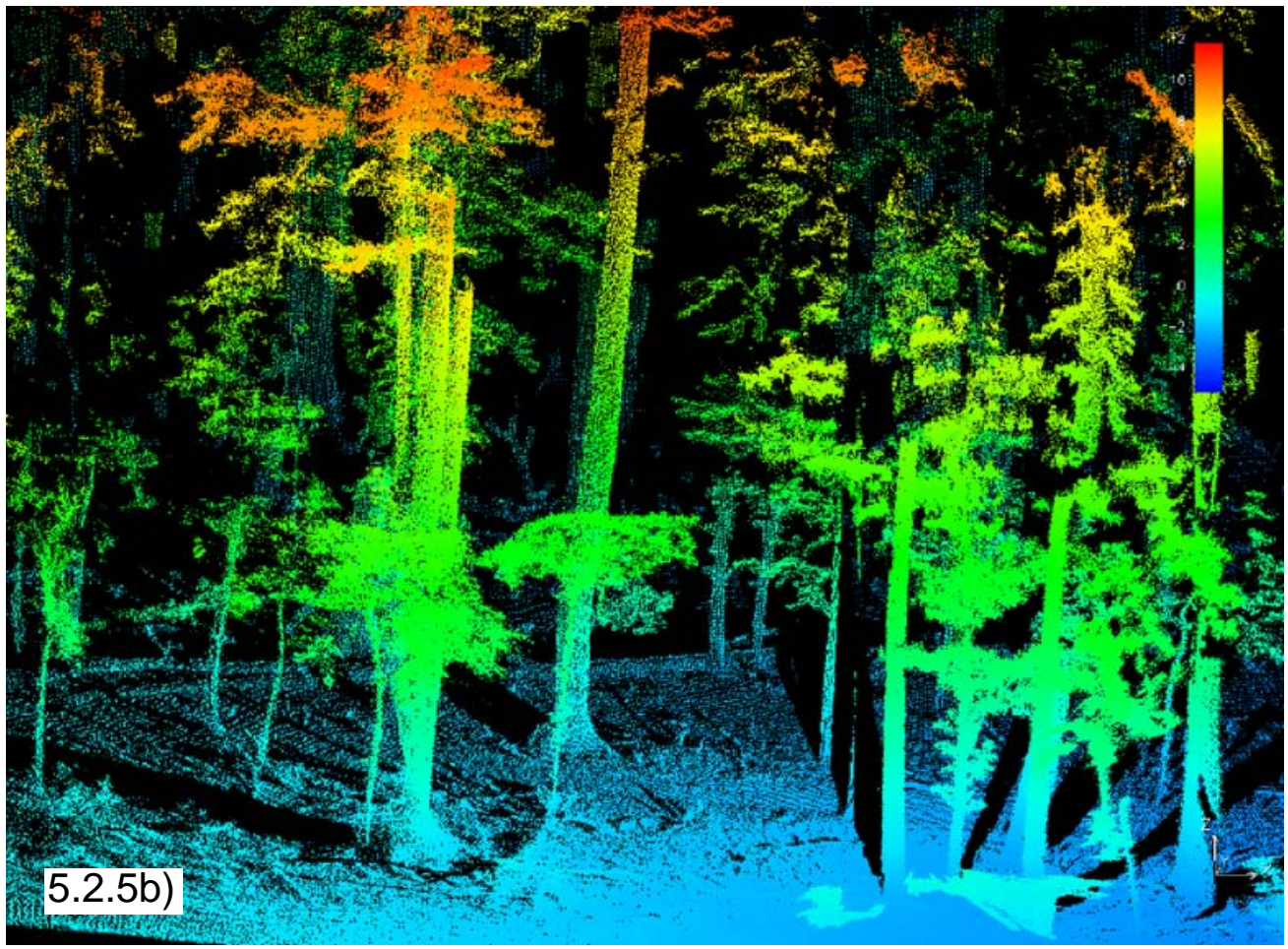


mass-production device, the image tube front end adds significant cost to the sensor and has a finite lifetime.

The architecture for implementing an FPA-based LADAR must be designed to handle a flood of data. A $128 \times 128$ pixel array generating 16 bit range information at $30 \mathrm{~Hz}$ produces a data bus load of a megabyte/s. Typically this is handled by a commercial "frame grabber" chip. This is assuming that only one range value is reported per pixel. There are situations (e.g. Figure 2.1.5) where this is not the case and one finds the entire time history to be of significance. Figure 2.1.6 showed what such a time domain response might look like where there exist multiple returns of interest within a single pixel response. If we are working at a nominal $100 \mathrm{~m}$ range and the accuracy (bin size) required is $75 \mathrm{~mm}$, then we must sample each pixel at $2 \mathrm{GHz}$. For a $30 \mathrm{~Hz}$ system, then, there is an 80 kilobyte/s bandwidth that must be handled in the off-loading of the data per pixel (assuming 2 bytes/sample and approximately 1300 range bins). Automated thresholding techniques can be used to reduce this required bandwidth (reporting only "hits" that register above the noise threshold). A fast, dedicated FPGA (field programmable gate array) can then be used to handle multiplexing and readout. Figures 5.2.4 and 5.2.5 show imaging results from a typical "brass board" implementation of an FPA LADAR.

\section{$\underline{5.3 \text { APD Solutions }}$}

Work at MIT Lincoln Lab has taken yet another approach to FPA development. Rather than using image tubes to achieve enhanced impact ionization they have developed a "geiger-mode" avalanche photodiode (APD) array that is integrated with fast CMOS time-to-digital converter circuits. The particular detector designed for this work is responsive to the arrival of single photons. When a photon is detected there is an explosive growth of current over a period of tens of picoseconds. The advantage to this approach is that smaller, more compact and lower power eye-safe laser illumination sources can be used. Lincoln has strongly promoted the "geiger mode" detector approach in the LADAR community and it is worth distinguishing here the difference between photodiodes, avalanche photodiodes, and "geiger-mode" APDs as these all are candidate approaches for Next Generation LADAR systems. Aull et al [2001] provides a useful discussion of this distinction:

$P-N$ Photodiode: An avalanche photodiode is a variation of a $p-n$ junction photodiode. When a $p-n$ junction diode is reverse biased, an electric field exists in the vicinity of the junction that keeps electrons confined to the $n$ side and holes confined to the $p$ side of the junction. When an incident photon of sufficient energy $(>1.1 \mathrm{eV})$ in the case of silicon) is absorbed in the region where the field exists, an electron-hole pair is generated. Under the influence of the field, the electron drifts to the $n$ side and the hole drifts to the $p$ side, resulting in the flow of photocurrent in the external circuit. The time integral of the current can be shown to be one electron charge. An electron-hole pair can also be thermally generated, resulting in leakage current, which is also called dark current because it is present even in the absence of incident light. 
The drift velocity, for high electric fields in silicon, is about $107 \mathrm{~cm} / \mathrm{s}$ for both electrons and holes. When a photodiode is used to detect light the number of electronhole pairs generated per incident photon, a metric known as quantum efficiency, is at best unity. Losses due to reflection or absorption in zero-field regions usually lower [sic] the quantum efficiency.

Linear Mode APD: An APD detects light using the same principle. The difference between an APD and a p-n junction photodiode is that an APD is designed to support high electric fields. When an electron-hole pair is generated by photon absorption, the electron (or the hole) can accelerate and gain sufficient energy from the field to collide with the crystal and generate another electron-hole pair, losing some of its kinetic energy in the process. The process is known as impact ionization. The electron can accelerate again, as can the secondary electron or hole, and create more electron-hole pairs, hence the term "avalanche." After a few transit times, a competition develops between the rate at which electron-hole pairs are being generated by impact ionization and the rate at which they exit the high-field region and are collected. If the magnitude of the reverse-bias voltage is below a value known as the breakdown voltage, collection wins the competition, causing the population of electrons and holes to decline. This situation represents the most commonly known mode of operation of APDs: measuring the intensity of an optical signal and taking advantage of the internal gain provided by impact ionization. Each absorbed photon creates on average a finite number $\mathrm{M}$ of electron-hole pairs.

Because the average photo-current is strictly proportional to the incident optical flux, this mode of operation is known as linear mode.

Geiger Mode APD: This effect is achieved by reverse-biasing the APD above the breakdown voltage using a power supply that can source unlimited current. When the reverse bias exceeds the breakdown voltage, the electrons and holes multiply

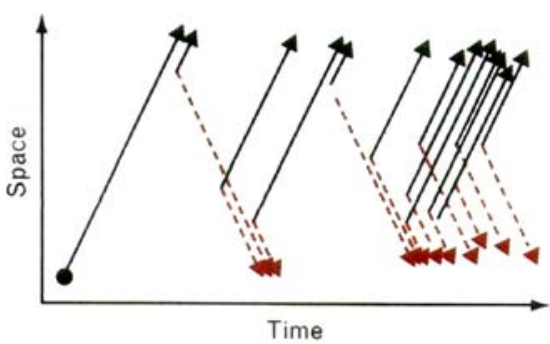

Figure 5.3.1 In Geiger-mode, in which the APD is biased above the avalanche breakdown voltage, the growth in the population of electrons and holes due to impact ionization outpaces the rate at which they can be extracted, leading to exponential growth of current. Courtesy MIT Lincoln Lab. by impact ionization faster, on average, than they can be extracted. This is the best definition of avalanche breakdown voltage. Figure 5.3.1 shows this concept. The population of electrons and holes in the high field region and the associated photocurrent grow exponentially in time. The more above breakdown the APD is biased, the faster the growth time constant. This growth of current continues for as long as the electric fields in the device are negligibly altered by the presence of the growing current and the growing population of electrons and holes. If there is a series resistance in the diode, however, more and more voltage is dropped across that resistance as the current grows. This effect reduces the voltage dropped across the high-field region, and 
therefore slows down the rate of growth of the avalanche. Ultimately, a steady state condition is reached where the voltage across the high-field region is reduced to the breakdown voltage, where the generation and extraction rates balance. At this point the current neither grows nor decays, and the series resistance provides negative feedback that tends to stabilize the current level against fluctuations. A downward fluctuation in current, for example, causes a decrease in the voltage drop across the series resistance, and an equal increase in the drop across the APD high-field region, which in turn increases the impact-ionization rates and causes the current to go back up. If the level of steady-state current is not too small (less than a few tens of microamps), it continues to flow indefinitely. Therefore, an avalanche initiated by the absorption of a single photon causes the diode current to grow to some resistance-limited value. The turn-on transient of this current is fast, typically lasting tens of picoseconds.

Geiger-Mode Quenching: Simply connecting an APD to a low-impedance power supply gives no way to either detect the turn-on or shut-off of the avalanche so that the APD is ready to detect another photon. Shutting off the avalanche current is called quenching, and is accomplished by two types of circuit techniques. In passive quenching circuits (see Figure 5.3.2), the APD is charged up to some bias above breakdown and then left open circuited. Once the APD has turned on, it discharges its own capacitance until it is no longer above the breakdown voltage, at which point the avalanche dies out.

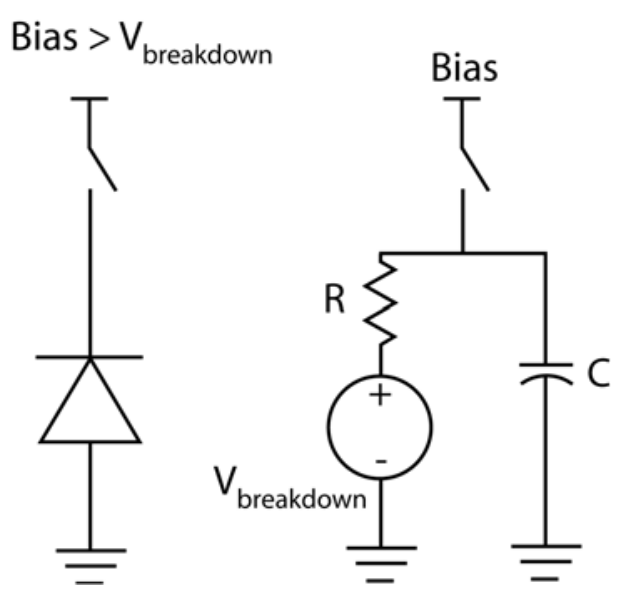

Figure 5.3.2: In Geiger mode (left) the APD is charged up to some bias above the breakdown voltage $V$ and then left open circuited.

Subsequently, once the avalanche has been initiated, the APD behaves according to a simple circuit model (right). Courtesy MIT Lincoln Lab.
Once the avalanche has been quenched, the APD can be recharged through a switch transistor. With passive quenching, the count rate will saturate at low optical fluxes because many photons will arrive when the APD is partially or fully discharged, and therefore unresponsive. Active quenching circuits, in contrast, uses a circuit to sense when the APD starts to self-discharge, and then quickly discharges it to below the breakdown voltage with a shunting switch. After sufficient time to quench the avalanche, it then recharges the APD quickly by using a switch. With a fast active quenching circuit, the APD can be reset after each detection on a time scale as short as nanoseconds, enabling it to function as a photon counting device at much higher optical intensities." 
Figure 5.3.3 indicates that relatively high photon detection efficiencies (probability of detection of a single photon) can be achieved in Geiger-mode operation of an APD through the use of high over-bias voltages.
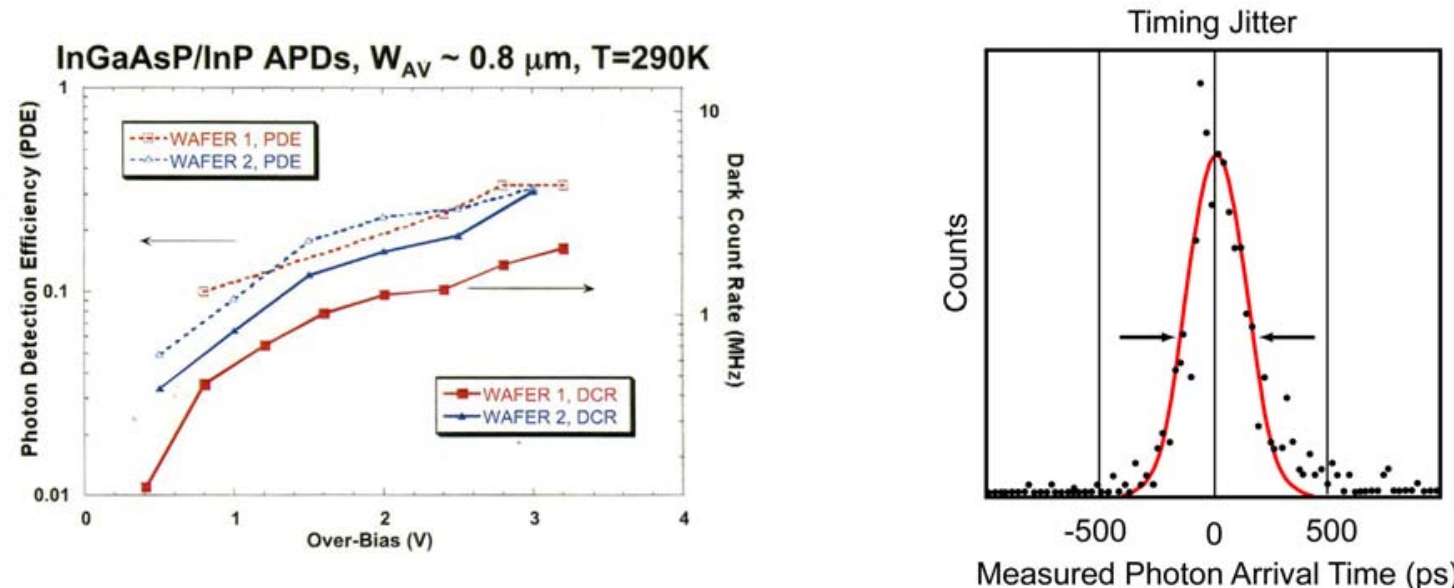

Figure 5.3.3: In Geiger mode the APD is charged up to some bias above the breakdown voltage $V$ and then left open circuited. The photon detection probability is shown at left as a function of the over bias voltage. A photon detection probability of 1 would imply a valid measurement for each photon received. The figure at right shows measured photon detection times obtained by repeatedly illuminating an APD with a series of laser pulses of 250 ps duration, attenuated so that the probability of detection is low. The width of the curve is due to a combination of the duration of the laser pulse and the timing jitter of the APD. This curve implies an accuracy error budget of $+/-45 \mathrm{~mm}$ (1 sigma) due to these factors alone. Other elements (e.g. digitizing clock speed) add further to the error budget. (Courtesy MIT Lincoln Lab).

The limiting accuracy of a time of flight measurement in a LADAR is controlled at the APD level by the statistical variation of the time interval between the pulse arrival and the resulting electrical signal from the APD. Lincoln Lab refers to this variance as "timing jitter" and it is associated with a number of error sources including: finite laser pulse duration combined with photon detection probabilities less than 1 infers that the detected return pulse can occur at any point along the received pulse shape; the APD response time is dependent on how deeply (energetic) the impacting photon reaches within the silicon - and the absorption length increases with wavelength; statistical variation in the growth of the avalanche current; and thermal noise due to APD resistance. The approach developed at Lincoln has limited "timing jitter" to +/- 150 ps. Further details on this subject are available in Aull, et al. 2001.

\subsection{1 $\underline{\text { Timing }}$}

The crux of any FPA LADAR design lies in the extraction of the time-of-flight. In the case of direct detection techniques, this means developing a fast clock and a means for reading out the time for each pixel. Previously, we have described simple approaches for single detector scanned systems (e.g. Schilling, Barr et al., 2000) in 
which the output of a telecom APD is directly fed to a high speed digitizer. In FPA design, all of this "pixel circuitry" has to go on the detector chip itself, or in a bumpbonded ROIC chip in a plan form that is no larger than that of the detector pixel. In actual practice, it is the timing circuitry that practically limits the array size. Figure 5.3.4 illustrates the concept of the "unit cell" or pixel timing "pipeline". Mastery of this concept means getting the highest timing resolution in the smallest space while prohibiting "cross talk" among neighboring pixels.

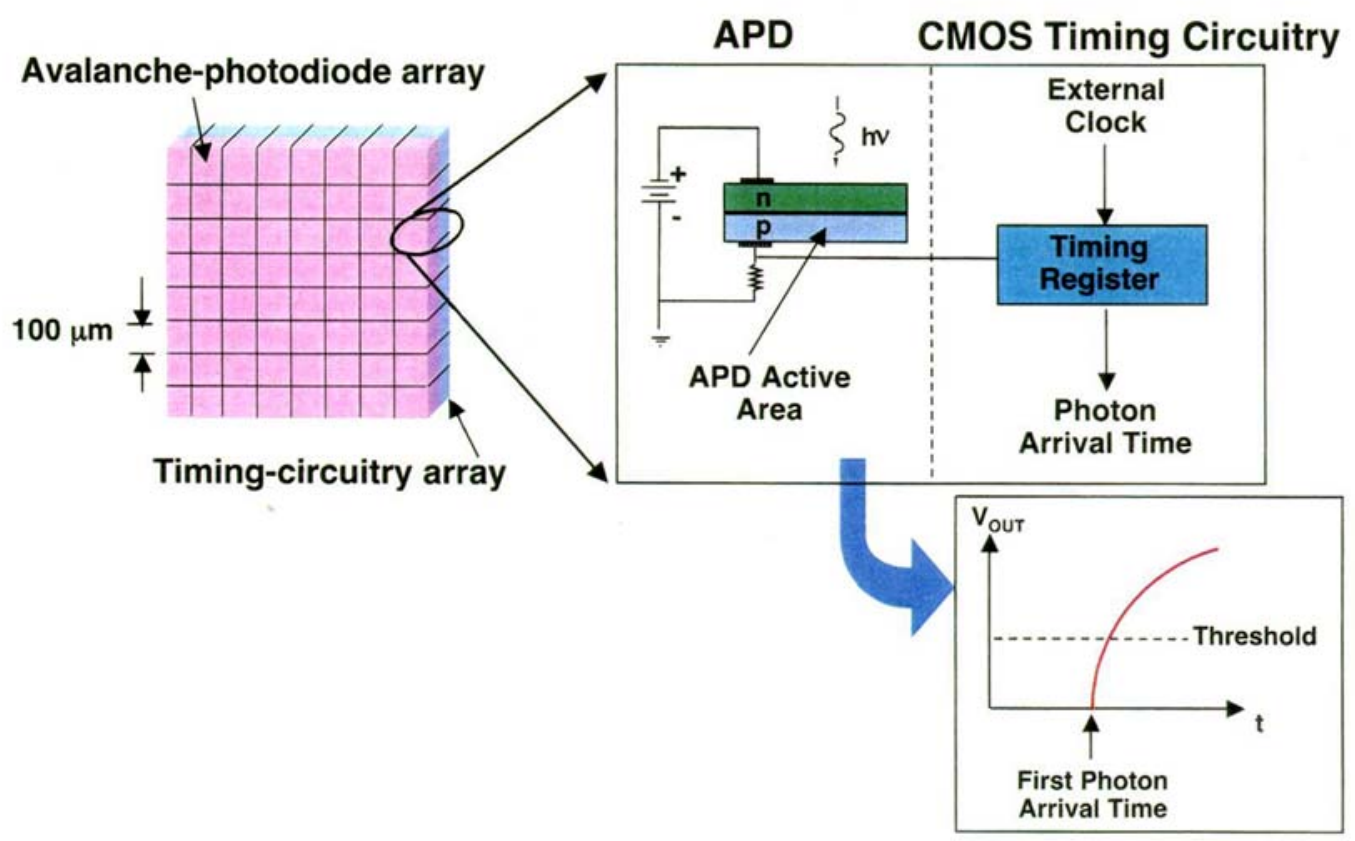

Figure 5.3.4: Basic structure of focal-plane array pixel circuit (unit cell) consisting of Geiger-mode avalanche photodiodes bonded to CMOS timing circuitry. In this architecture, the first detected photoelectron triggers an avalanche - a large current pulse; the current pulse, corresponding to a valid object hit above some predefined threshold, latches the timing register; the register is then decoded and the range stored. Courtesy MIT Lincoln Lab.

The concept of pixel timing circuitry is important enough to NGL that the majority of the remainder of this chapter is devoted to the subject. The initial approach taken by Lincoln Lab is shown in Figures 5.3.5 and 5.3.6 which show a block diagram and circuit schematic, respectively of their prototype timing circuit. Aull et al, 2000 explains the operation of this circuit as follows:

[The approach] consists of a pseudorandom counter clocked by a $500-\mathrm{MHz}$ master clock that is broadcast to all pixels. The pseudorandom counter is a shift register with a feedback path that has an exclusive OR gate; if the shift register is $n$ bits long, it cycles through a sequence of $2^{\mathrm{n}}-1$ distinct states. The clock is fed to the counter through a transparent latch whose output is frozen when the photon is detected. The state of the counter thus encodes the number of clock cycles that 
elapsed from the start of counting to the photon detection time. The state of the transparent latch, which is also read out, indicates whether the photon was detected in the high-clock or low-clock portion of the clock period. Thus 1-ns photonflight-time resolution is achieved. By generating a secondary clock delayed by 90 degrees with respect to the master clock and storing its state in a second transparent latch, an additional vernier bit is generated, and 0.5 ns resolution is then achieved. In LADAR applications this approach yields a range resolution of $76 \mathrm{~mm}$ (but see the notes above regarding timing jitter).

The pseudorandom count value must be decoded by table lookup or reverse encoding after it is read out. The benefit of this implementation is that it gives a compact pixel circuit; a conventional binary counter would require much more chip real estate. The vernier bit scheme facilitates achievement of sub-ns precision without requiring $2 \mathrm{GHz}$ clocking. Therefore, it is possible to use an established commercial foundry process with $0.5 \mu \mathrm{m}$ or $0.35 \mu \mathrm{m}$ gate geometries.

The Lincoln Lab approach uses a Geiger-mode APD to serve as a photon detector. When it detects a photon there is an explosive growth of current - over a period of around tens of ps with timing "jitters" on the leading edge of the current buildup of tens of ps. A circuit that could sense the rise of the photocurrent when the APD fires might have something inherently capable of yielding the mm-level accuracies sought for NGL. But the question is: how do you sense that current rise? In the Lincoln approach they are driven by making FPAs. They are not so driven to achieve enhanced timing for an APD since $76 \mathrm{~mm}$ resolution has been sufficient for

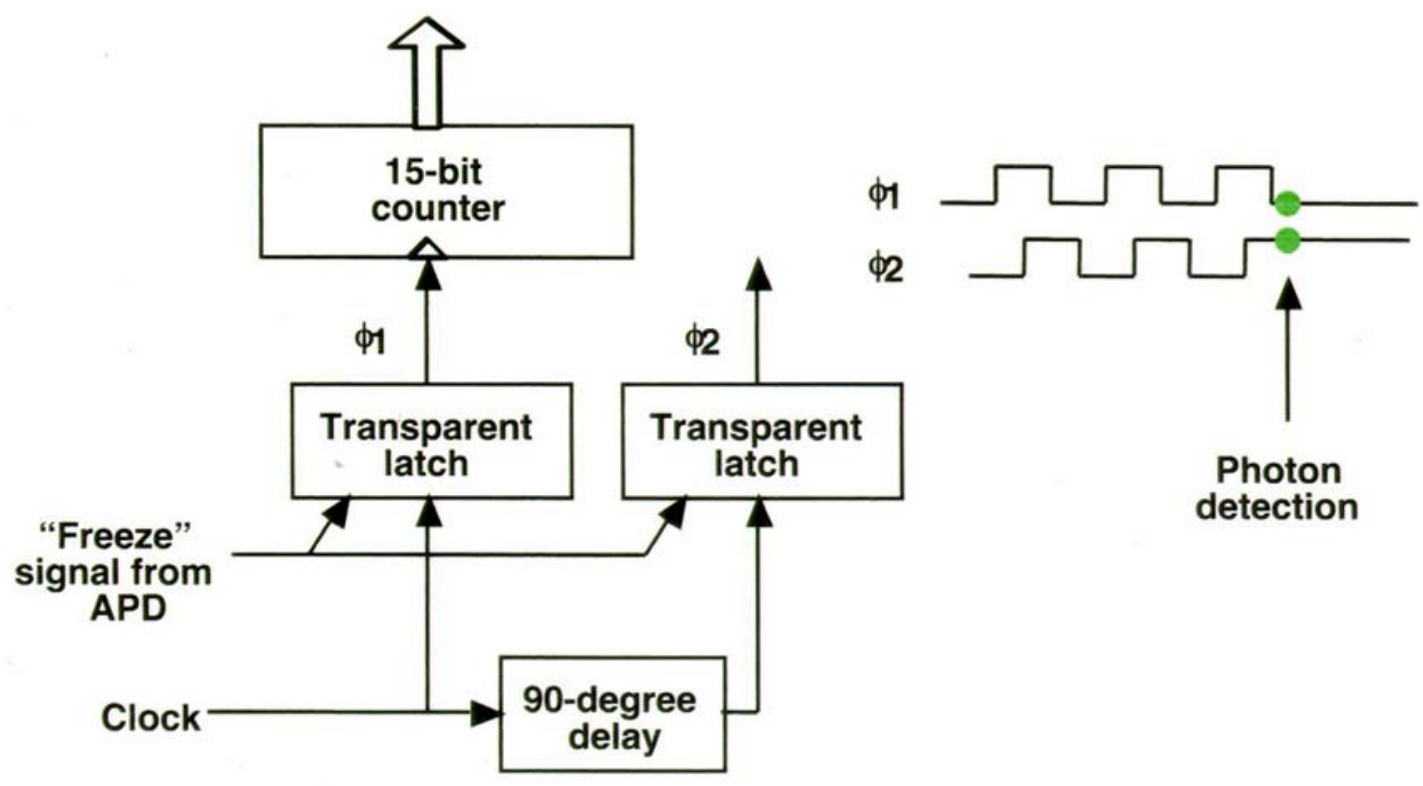

Figure 5.3.5: FPA Timing circuit block diagram showing the method by which a vernier bit is obtained to improve range precision. Courtesy MIT Lincoln Lab. 
their design purposes. The present Lincoln approach is basically a photon-counter. One could, alternatively, proceed directly to an APD connected to a pixel circuit. If you really want to sense the photocurrent event in the APD there are good circuits to do that, but generally they are analog circuits (see Section 5.4 below).

Lincoln was interested in a photon counting approach with a compact simple pixel circuit. They did the simplest thing possible and developed an APD to a CMOS inverter. In that format you sense the voltage, not the current, which means that you have to wait longer for the current to build up, saturate, and then discharge the capacitance across the APD to below breakdown. That event -- the time for the APD to discharge its voltage -- is a longer time scale than the initial buildup of the current. When you move to voltage sensing you introduce a timing latency on the order of ns rather than ps. The advantage is that it is a simple pixel circuit, and by careful choice of the bias voltage on the APD one can achieve a photon-to-digital converter where no analog circuitry in the pixel is needed.

It is important to recognize that in the above approach (and the one described in Figures 5.3.7 and 5.3.8) that the entire waveform from the APD is not being digitized (in contrast, see section 5.4). The output of the APD is connected to a thresholding element. The circuit senses when the voltage on the APD drops below a certain threshold. When the APD turns on it looks like an RC circuit, that is, a resistance discharging a capacitor. The APD is charged up to $5 \mathrm{v}$, a photon hits the APD and turns it into a $20 \mathrm{~K}$ (ohm) resister that starts discharging the capacitor. Typically the RC time constant of that combination is of ns duration (not ps). One can easily get "jitter" in the hundreds of ps just because of the simplicity of the circuit. Unlike some approaches, there is no oversampling of the entire waveform.

To obtain time-of-flight, Lincoln Lab uses an ordinary telecom APD next to the laser to provide an optical trigger (to start the clock). In each pixel of the FPA they also have a digital counter... a stopwatch...on the CMOS readout chip. The master clock is created using a ring oscillator. This can be created using a circular chain of an odd number of inverters. For example, one could take nine inverters and connect them into a circle. Logically, this is an inconsistent circuit. If you assume logic 0 on output and then trace your way around to the same place -- the same node in the circuit -- you arrive at the conclusion that it has to be logic 1 . So the circuit is not stable and it oscillates, producing a clock whose period is the time to make two round trips around the chain of inverters. This is a standard means of generating a clock. In the Lincoln Lab approach (Figures 5.3.7 and 5.3.8) one of the inverters is replaced with a NAND gate. The second input to the NAND gate is a gating signal to turn the clock generator on. So this is a circuit that produces a clock that can be turned on remotely -- at some designated time (by the light pulse sensed upon laser firing). Regarding the triggering of the clock, there are potential problems that arise due to the nature of the illumination source. If, as in the case of the Lincoln Lab design, a microchip laser is used, then one can generate significant jitter (due to timing lag) from the command signal that pumps the pump laser and the actual time that the light comes out 


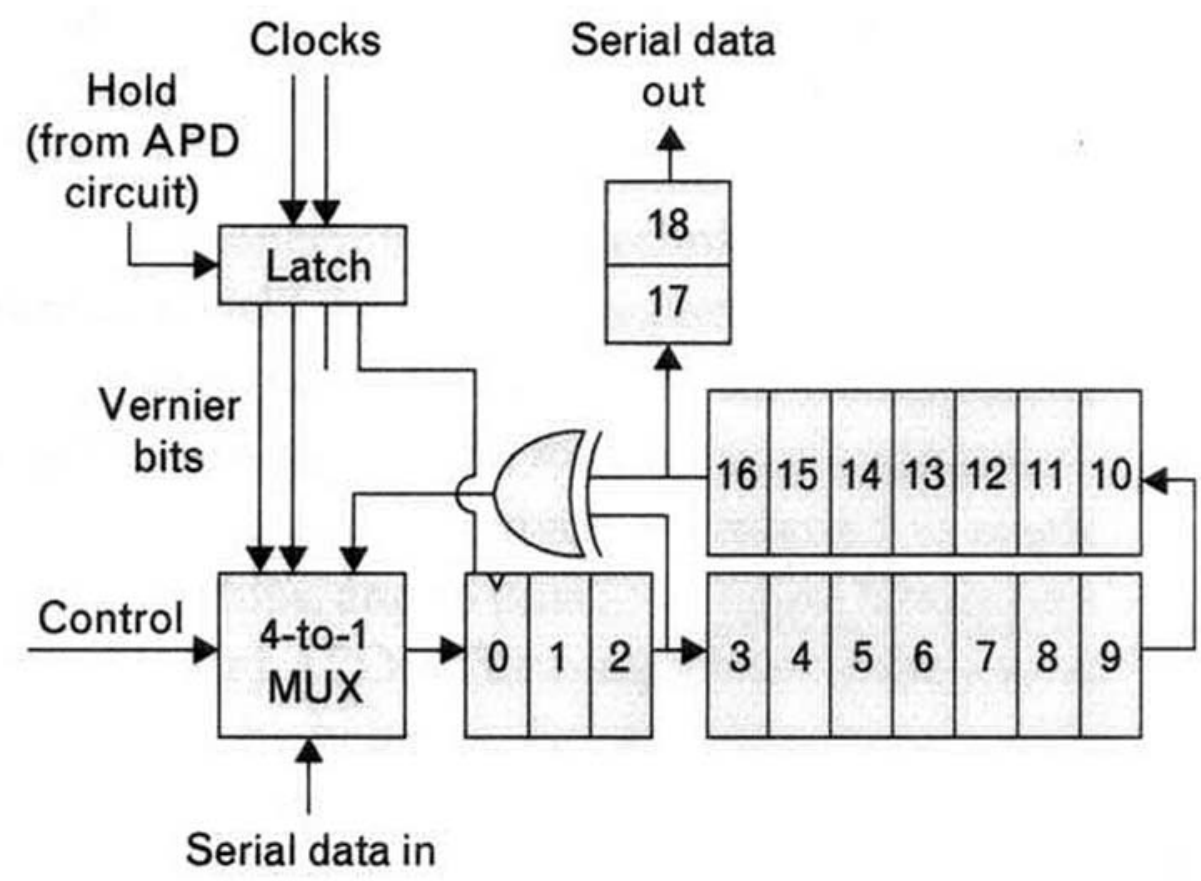

Figure 5.3.6: Block diagram of the pixel circuit of the timing chip, which functions as stopwatch, timing the detection of the photon by the APD. A 17-bit shift register with an exclusive OR (XOR) feedback loop is a pseudorandom counter. Photon detection causes the clocks that drive the counter to freeze. The counter and the stored states of the clocks encode the time of detection. (Courtesy MIT Lincoln Lab.

(microchip lasers typically use pump laser diodes to provide the lasing energy for the primary laser chip). Thus, to avoid this timing lag you have to sense the beginning of the lasing pulse output optically and use that as the trigger signal to start the clock. Traditionally this is done with a beam splitter. It could also be accomplished by using scattered light from the optics array itself. If the illumination source is, instead, a diode laser, then the requirement for optical sensing diminishes since the delay between the pump circuit charging and actual lasing is on the order of a few ps.

The CMOS clock signal on chip is broadcast to all the pixels in the FPA. This starts clocking the digital counters in each pixel. Each pixel's counter is controlled by this master clock. Within each pixel the clock goes to the counter through another logic element that is gated by the signal from the APD. This additional logic element is a tri-state inverter -- a logic element that has three possible states: 1) 0 and invert to 1 ; 2) 1 and invert to 0 (a normal inverter); and 3) freeze whatever logic state is on the output, e.g., if 1 , hold that 1 and don't listen to other input; if 0 , hold that. So the tristate inverter has input, output, and a control signal that says "invert" or "freeze." In each pixel the APD, when it discharges, triggers an inverter that in turn goes to this input of the tristate inverter that tells it to freeze its output. The APD tells the tri-state inverter to store the current state of the clock. 


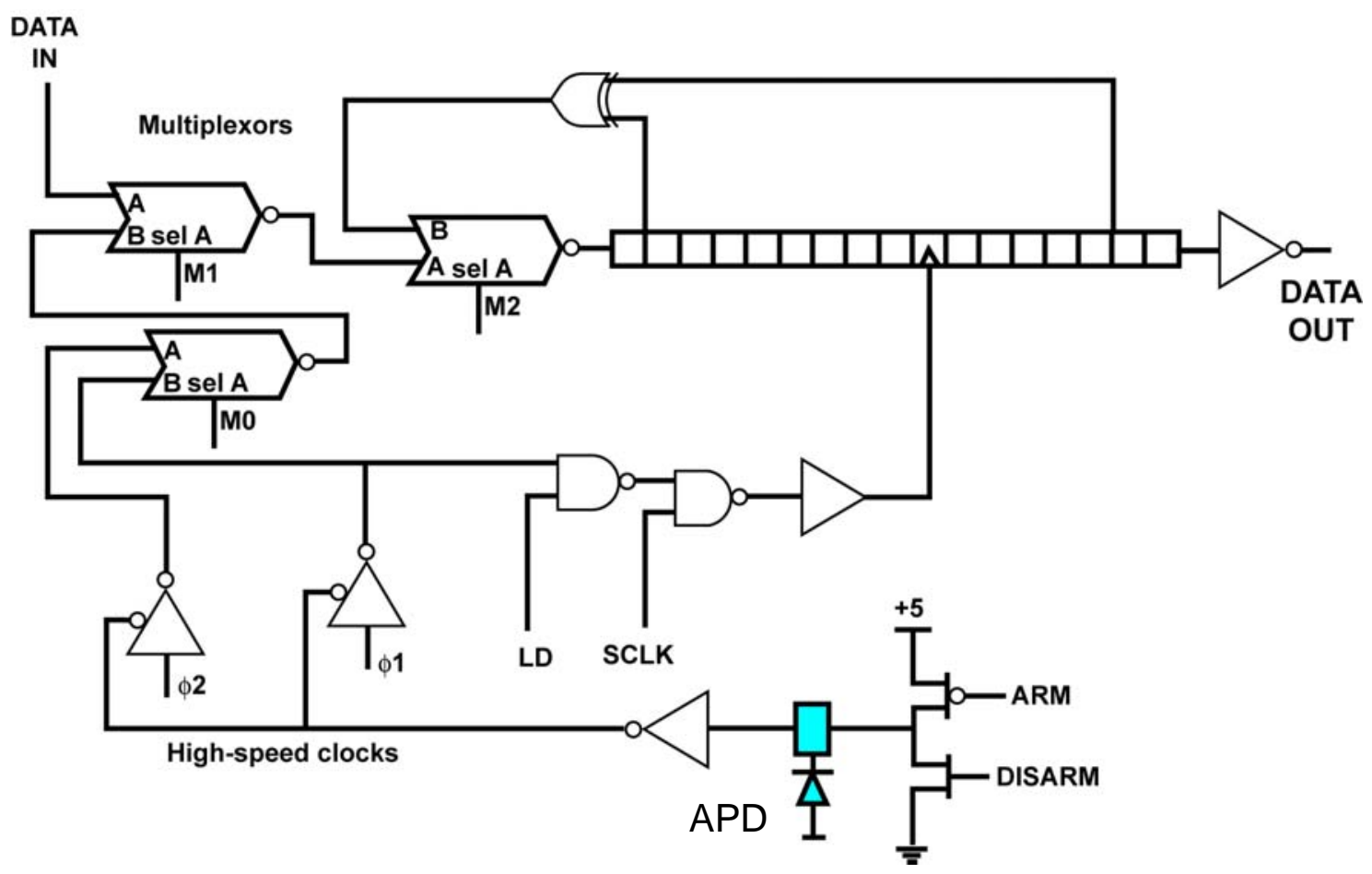

Figure 5.3.7: FPA Pixel (Unit Cell) Timing circuit schematic, 2001. Below: 2001 Master clock generator circuit. (Courtesy MIT Lincoln Lab).

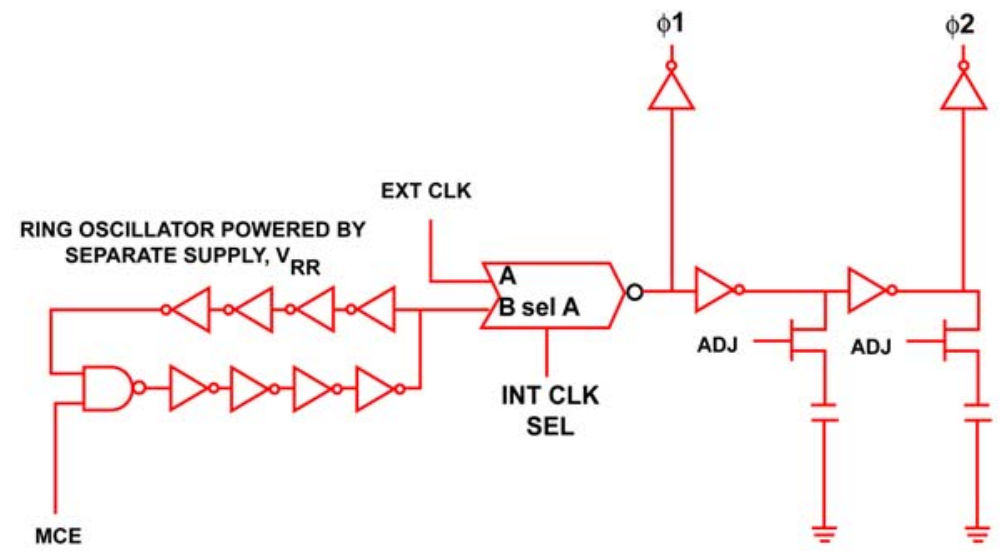

This does two things. First, it stops the counter, which is incremented by transitions of the clock. Now, stored in the counter in the pixel, there is a digital value that is the number of clock cycles since the generation of the transmitter start pulse. The counter circuit (see Figures 5.3.7 and 5.3.8) is a chain of flip flops. The flipflops store the digital values -- each comprising a small memory element. When clock pulses are applied to the string of flip flops, it increments. When clock pulse is stopped it holds the last value.

The accuracy of the system, "timing jitter" issues notwithstanding, is limited by the clock frequency -- purely the clock frequency. If the ring oscillator on chip generates a $500 \mathrm{MHz}$ clock, then the clock period, and hence the bin size, is $2 \mathrm{~ns}$. The flipflop 


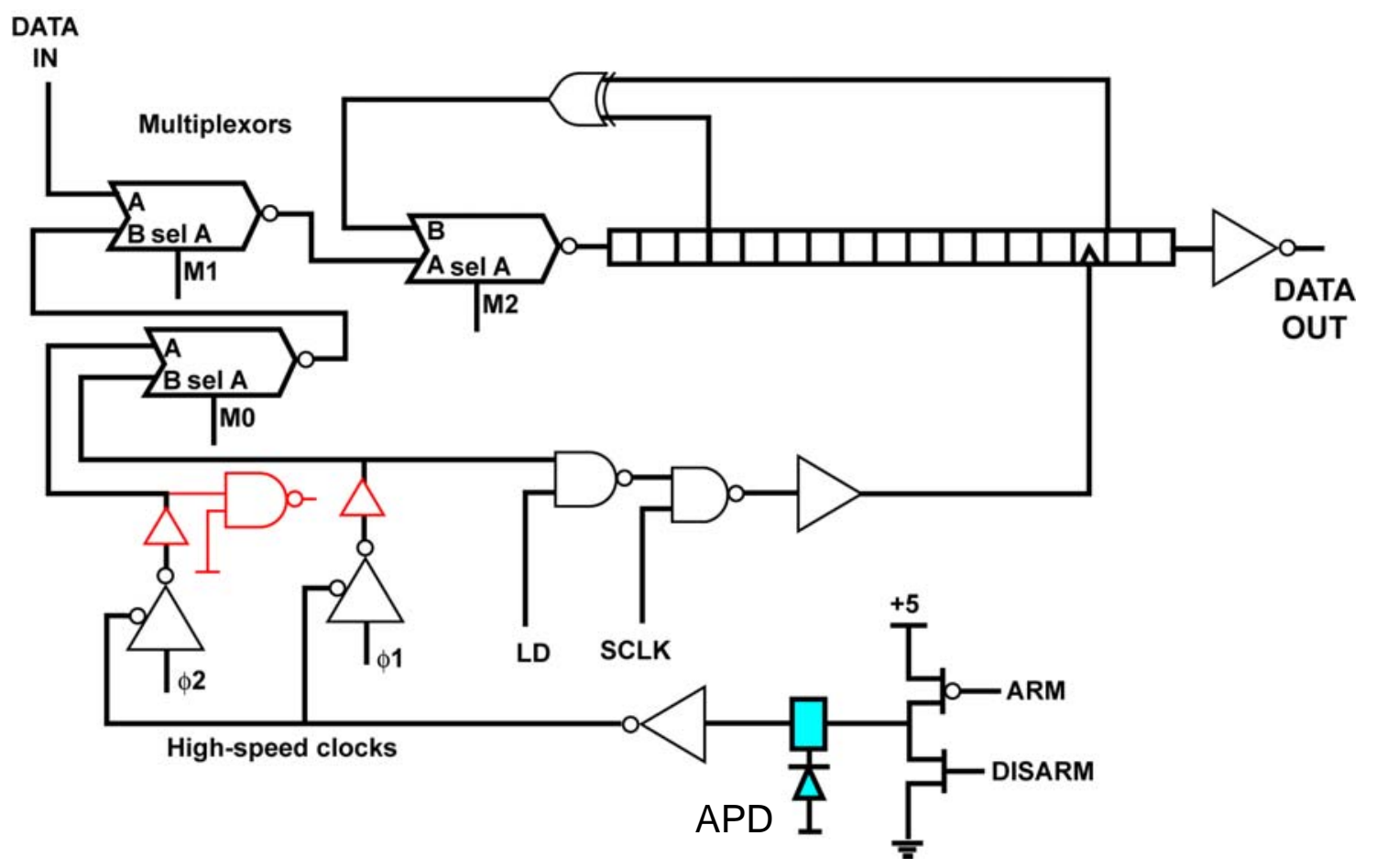

Figure 5.3.8: FPA Pixel (Unit Cell) Timing circuit schematic, 2002. Below: 2002 Master clock generator circuit. (Courtesy MIT Lincoln Lab).

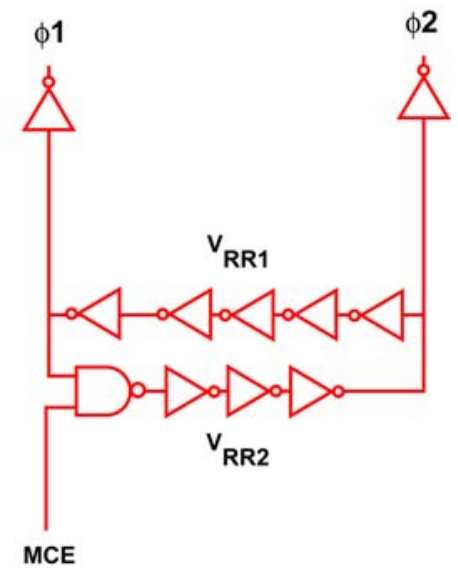

string counter in the pixel has thus stored a number that tells you the flight time (round trip) with a resolution of $2 \mathrm{~ns}$. Once the counter has been stopped, one can additionally inspect the output of the tri-state inverter to determine whether the clock was high or low when photon detection occurred. So you have an additional bit that tells what half of the clock cycle you were in. This additional bit increments on the rising or falling edge of the clock -- a square wave signal. As a further example, suppose the rising edge triggered the flip flops in the counter, which increment when you go from low to high and do nothing when you go from high to low. If you had only the counter you would know that the photon was detected in that particular clock period. But you would not know whether it happened during the high or low transition since the counter 
only increments on the rising edge. If the counter is "frozen" at some point in time then you know by reading out the counter how many clock cycles have elapsed. A string of flipflops is an unusual counter circuit -- it is essentially a shift register with a feedback loop, not a conventional counter, that stores a simple binary representation of clock count. In actuality, it goes through a sequence of states. If you have 15 flip flops the string will cycle through $2^{15}-1$ distinct states before returning to the starting state. When the flipflop string is read out it must be decoded into an actual timing value. This can be done most rapidly through a lookup table. The counter overflows (in range) when you reach $\left(2^{15}-1\right)^{\star} 2 \mathrm{~ns}$. But $2 \mathrm{~ns}$ is equal to $300 \mathrm{~mm}$ in range and thus 15 bits would provide an unambiguous range far in excess of any range of interest for NGL development. An 8 bit counter, on the other hand, would provide an unambiguous range of $\left(2^{8}-1\right)^{*} 300 \mathrm{~mm}=76.5 \mathrm{~m}$, which is in the correct range of interest. This reduction in flip flop count would serve to reduce required pixel real estate and lead to better form factor in an NGL device.

As described above, in this timing architecture the clock is gated off in each pixel following photon detection. There is thus only one timing value per pixel per laser pulse. There has been significant interest in the development of multiple-return per pixel designs, mainly driven by the DARPA JIGSAW program. Multiple timing values (per pixel) can be obtained with the above design by accumulating data over multiple laser pulses. Work is underway at Lincoln Lab to develop advanced pixel architectures in which the APD is rapidly reset following initial detection. Additional storage is available in the pixels for more than one timing value. Alternatively, one could assign multiple detectors to the diffraction limited pixel FOV. A 4x4 array assigned to a single pixel FOV would thus allow for 16 independent timing values (i.e. "hits" above threshold, not sequential bins) for a single illumination pulse.

\subsubsection{Fabrication}

Before leaving discussion of the Lincoln Lab approach it is worth discussing the methods used to fabricate the FPA since they are non-standard. Aull et al. [2001], explain:

The voltage requirements and the fabrication process requirements of a Geigermode APD are quite different from those of a CMOS logic process. A standard CMOS process cannot be used, forcing costly development of a specialized process that might necessitate compromises between circuit performance and detector performance. Second, a monolithic process most likely involves a partition of the pixel real estate into detector and circuit sections, thus limiting the fill factor of the detector array. In the long run, silicon-on-insulator technology and threedimensional integration techniques are likely to mature and yield monolithic implementations without these drawbacks. In the short run, our approach has been to use inexpensive foundry services to prototype the timing circuits, which are received as single unpackaged chips, and to pursue in-house fabrication of full wafers of APD's. The integration of the two is then achieved by bonding the chips face to face with the APD arrays, and illuminating the APD array from the substrate 


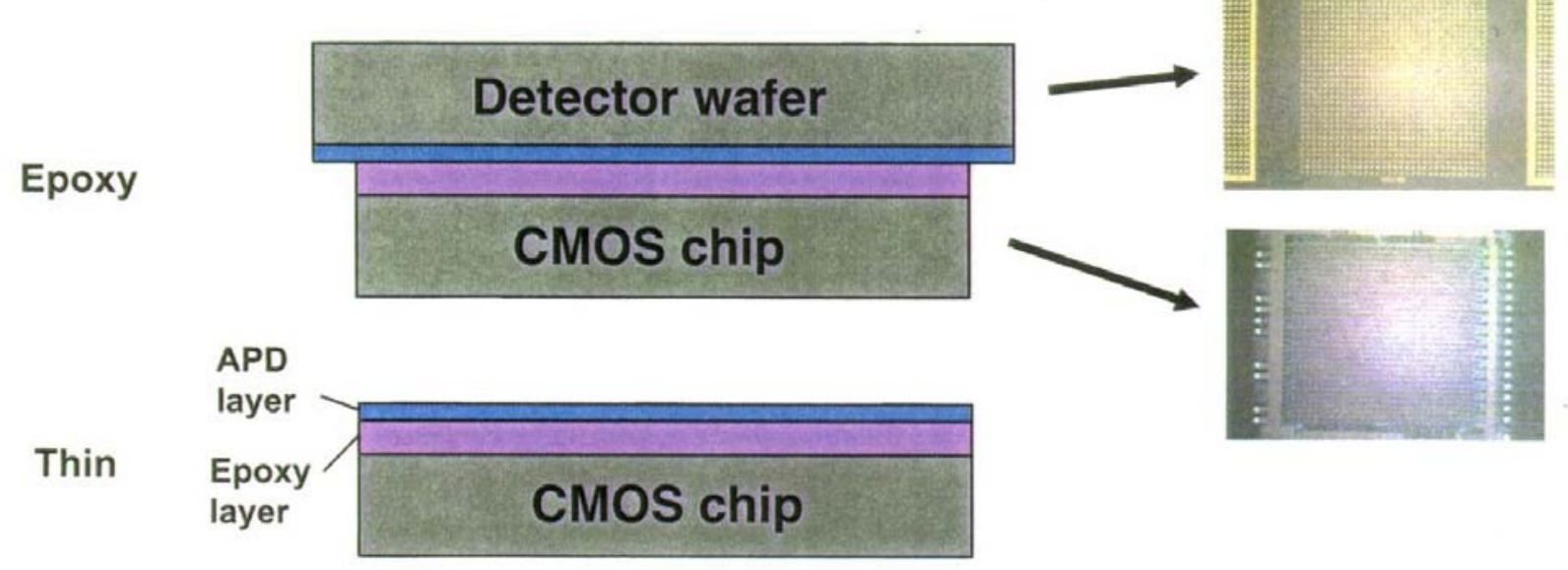

\section{Bridge bond}
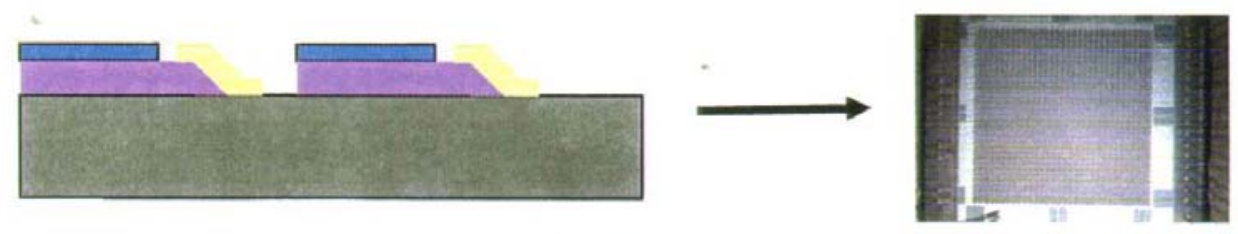

Figure 5.3.9: Bridge Bonding: The detector and ROIC wafers are epoxied together, and the APD substrate is removed through selective etching. Electrical connections are made by etching vias between the APDs and then depositing metal connections in those vias. (Courtesy MIT Lincoln Lab).

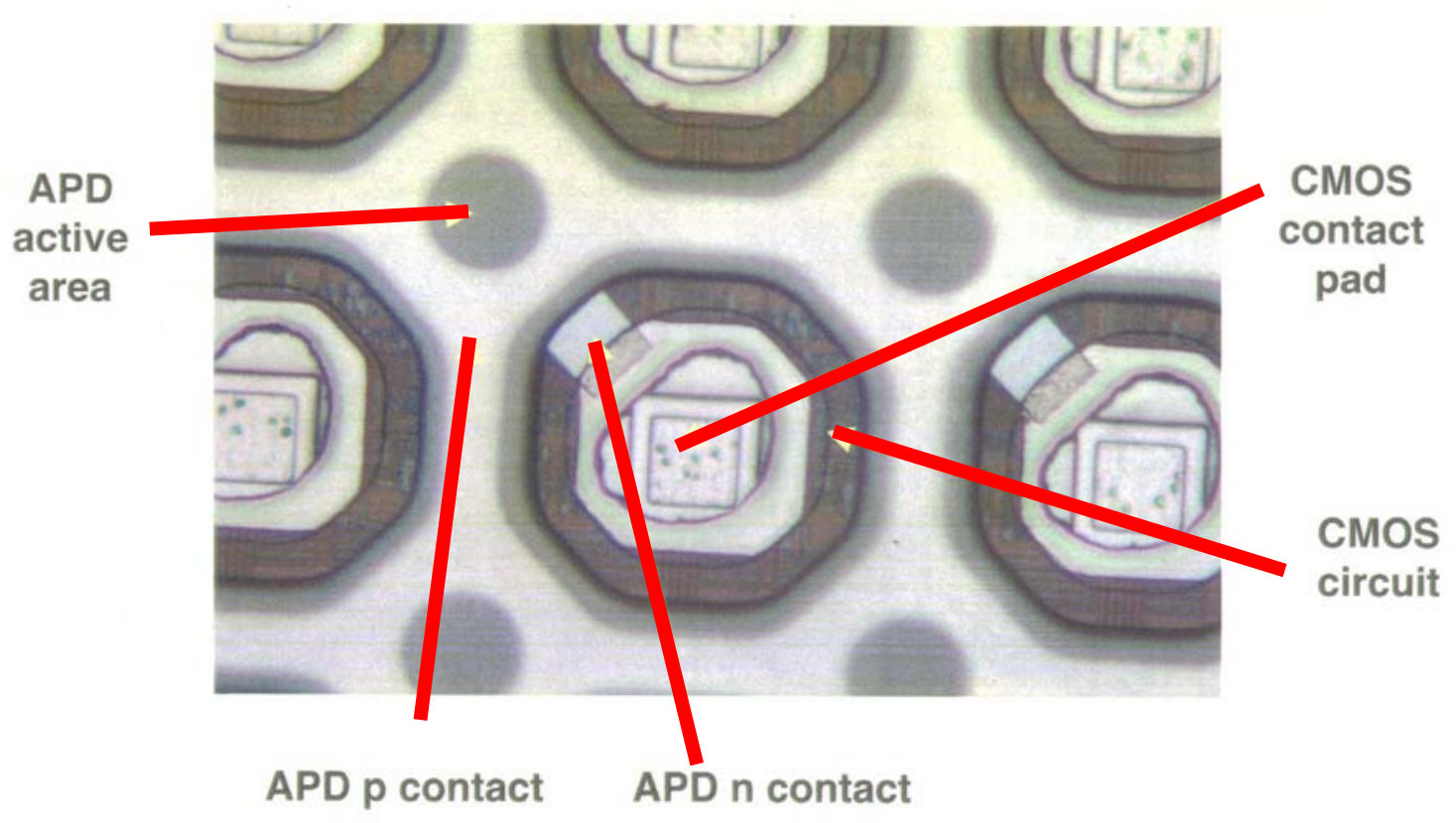

Figure 5.3.10: Photomicrograph of a bridge-bonded APD/CMOS device, showing features of the individual FPA pixels. (Courtesy MIT Lincoln Lab). 
side (so-called back illumination). ..... [The solution involves developing an] alternative process known as bridge bonding [see Figure 5.3.9 and 5.3.10]. First, the CMOS chip is epoxied face to face with the corresponding APD array without making any electrical connection whatsoever. The APD wafer, with several CMOS chips bonded to it, is epoxied, substrate up, to a handle wafer. The resulting APD/CMOS handle "sandwich" can then undergo the same types of clean-room fabrication processes that are applied to normal full wafers. The APD substrate is [then] removed. A shallow $p+$ implant and laser anneal is done on the exposed backside of the APD wafer in order to replace the p-side electrical contact layer formerly provided by the substrate. Vias are then etched in between the APDs and metal "bridges" patterned within the vias to connect each APD with the corresponding timing circuit. The backside of the APD is also metallized to provide a common electrical contact to the $p$ sides. In operation, this backside contact is held at a negative voltage slightly smaller in magnitude than the breakdown voltage, and the $n$ side of each APD is chartged up to a positive voltage (typically $4 \mathrm{v}$ or $5 \mathrm{v}$ ) by the pixel circuit.

Successful development of the bridge bonding process required overcoming a number of technological hurdles. Thinning must be uniform. Curing the epoxies used must not lead to destructive mechanical stresses due to the large thermalexpansion coefficient mismatch between semiconductors and epoxies. The vias through the epoxy must have sloped sidewalls to allow good step coverage of the bridge metal. Because of the vias, most of the required photolithographic steps are done on a nonplanar surface, which presents challenges of nonuniform photoresist thicknesses and exposure depth of focus issues. Finally, the handling of the APD must not result in excess increases in leakage current or dark-count rate.

\subsubsection{Compensating for Low Detector Fill Factor}

As can be seen in Figure 5.3.10, the chip real estate occupied by the APD detector active area is relatively limited in comparison to the chip area assigned to one pixel the remainder being occupied by etched vias, bridge bonds, and the CMOS contact pads and circuitry. A similar situation will arise for any single chip solution, e.g. as described in section 5.1. A $100 \%$ "fill factor" would imply that the APD occupied $100 \%$ of the usable chip surface (pixel area) exposed to the incident radiation from the laser illuminator. Although higher fill factors can be achieved by going with a more traditional indium bump-bonded ROIC, the area of the ROIC unit cell will then control the achievable pixel density to one similar to the approach described above. Alternate methods for achieving higher pixel density are discussed in sections 5.4 and 5.5 below.

Figures 5.3.11 and 5.3.12 show two commonly used methods to compensate for low fill factor. Each has advantages and disadvanges that depend on the application of interest. For longer range LADARs, where the FOV is likely to be small, one can use a microfabricated lenslet array (Figure 5.3.11 b). However, for short range, wide FOV applications, solar illuminated background can be an issue. One means of overcoming 


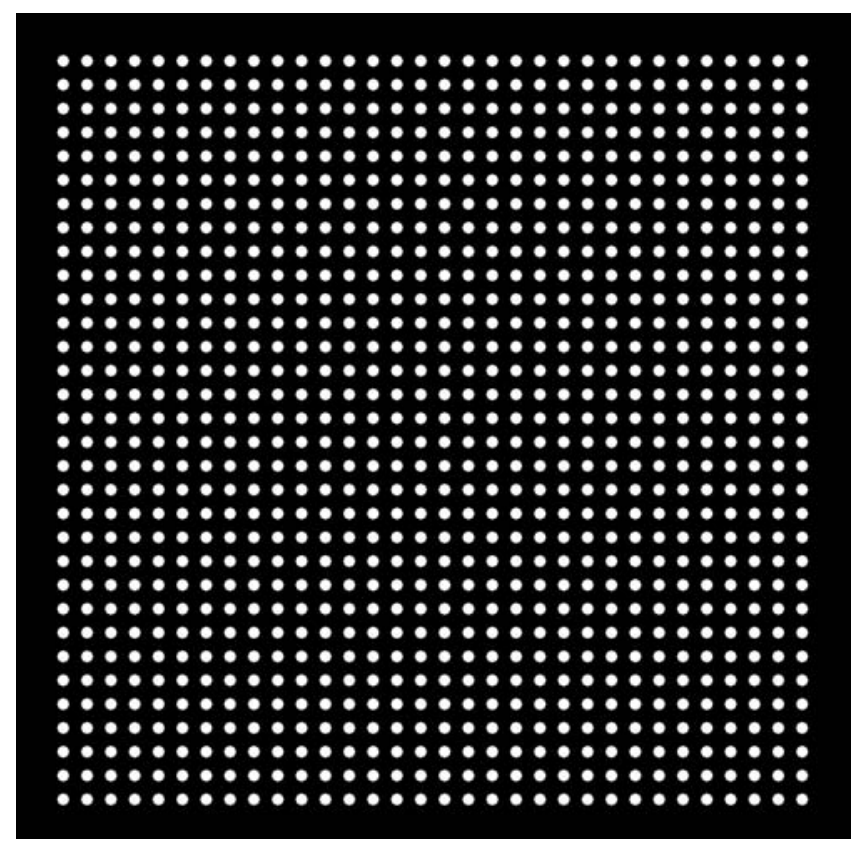

(a)

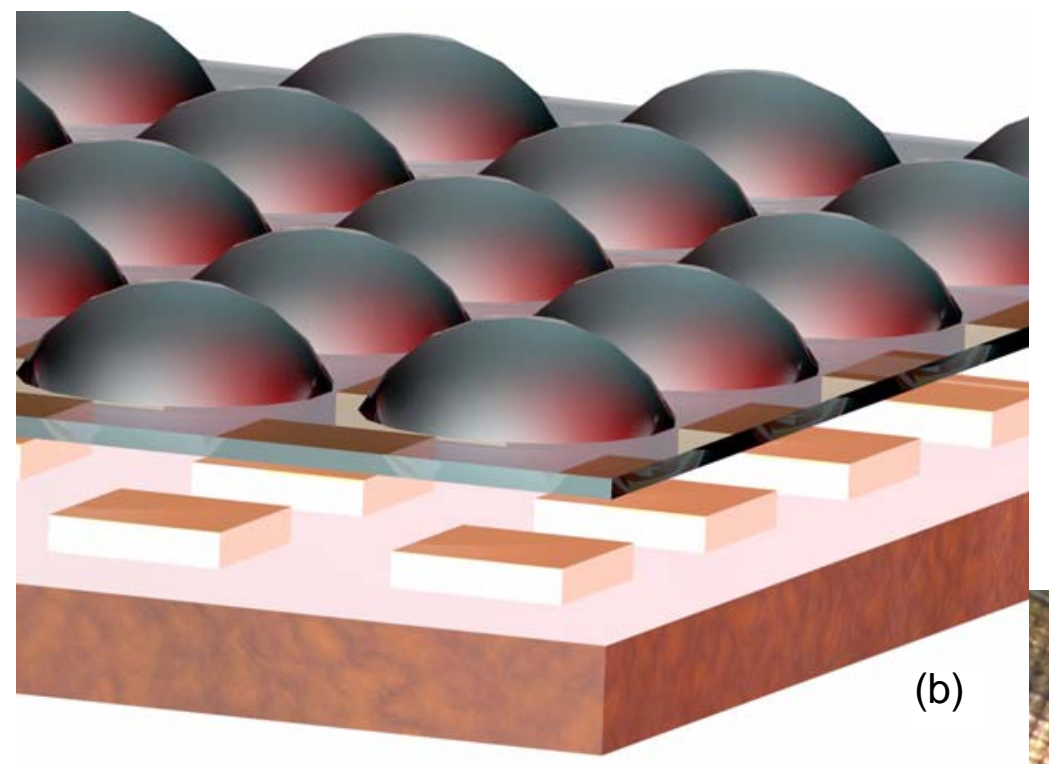

Figure 5.3.11: Two solutions to the problem of low fill factor. A transmitted spot array (a) has been proposed (and developed) by several organizations in concert with FPAs as a means of increasing the incident power (and hence signal strength) on the detector pixel. Such a pattern can be achieved through the use of binary diffractive optics at the output stage of the laser illuminator. Care must be taken to insure that the pattern FOV matches the receiver FOV for each of the detector pixels.

Yet another means of increasing the signal strength at the detector is to micro-fabricate a "lenslet" array (b) and mechanically assemble that with the detector FPA. In this manner a high percentage of the light impinging on each pixel area reaches the APD.

Figure 5.3.12 (right): Example of a $32 \times 32$ FPA with a lenslet array. One can see the moiré pattern of connection and active areas (compare to Figure 5.3.10.)

(Courtesy MIT Lincoln Lab)

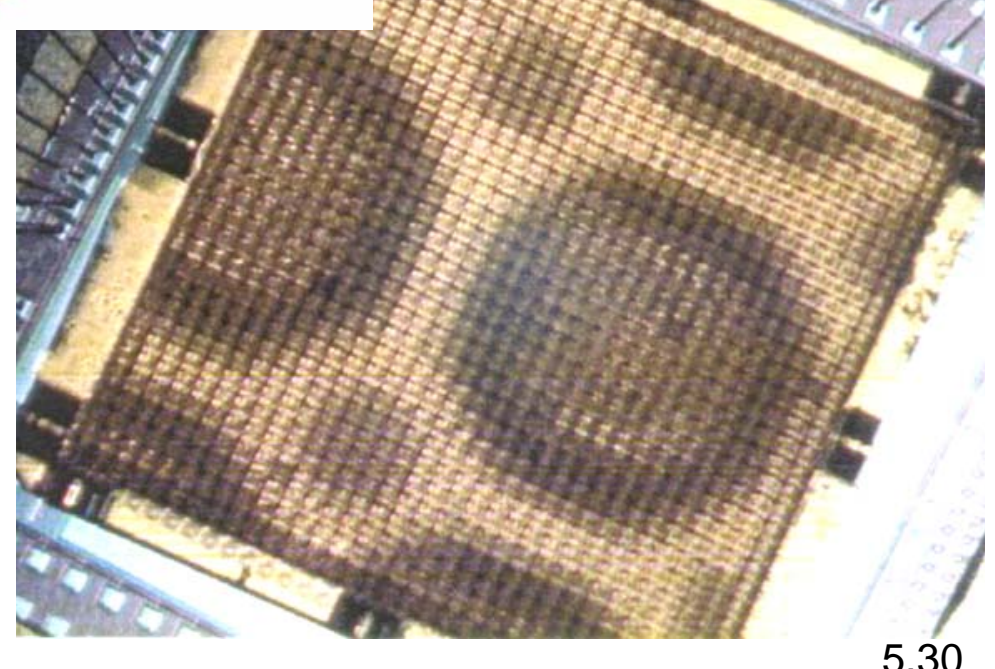


that is to focus a portion of the laser illumination towards each pixel FOV. In effect, the transmission beam is used to illuminate discrete locations associated with each pixel rather than to "area illuminate" as would normally be the case with staring active sensors. One can place binary diffractive optics in front of the laser transmitter to produce such a discrete spot array (see Figure 5.3.11a) that is optically mapped to each pixel. One can use this approach to obtain higher resolution in the illuminated spot areas.

\subsection{Z-Stacking and VISA Solutions}

The problem of limited real estate for the ROIC unit cell was recognized long ago when the first IR focal plane arrays were being developed for infrared imaging (not ranging) in the 1970s and 1980s. In those cases the ROIC was tasked with signal amplification, conditioning and image readout, not timing. But the concept remains the same. A novel solution that adds a third dimension to the available circuitry area is shown in Figure 5.4.1 and is known variously as "Z-technology," "Z-stacking," or simply "stacking" (e.g. see Carson [1975], [1984]; SPIE 1097).

In the chip-stacking ROIC concept, the unit cell does not occupy a finite planar area beneath, and parallel to, the detector plane and defined by the limits of the pixel area. Rather, the ROIC elements, which are on separate, thinned, IC wafers, are oriented

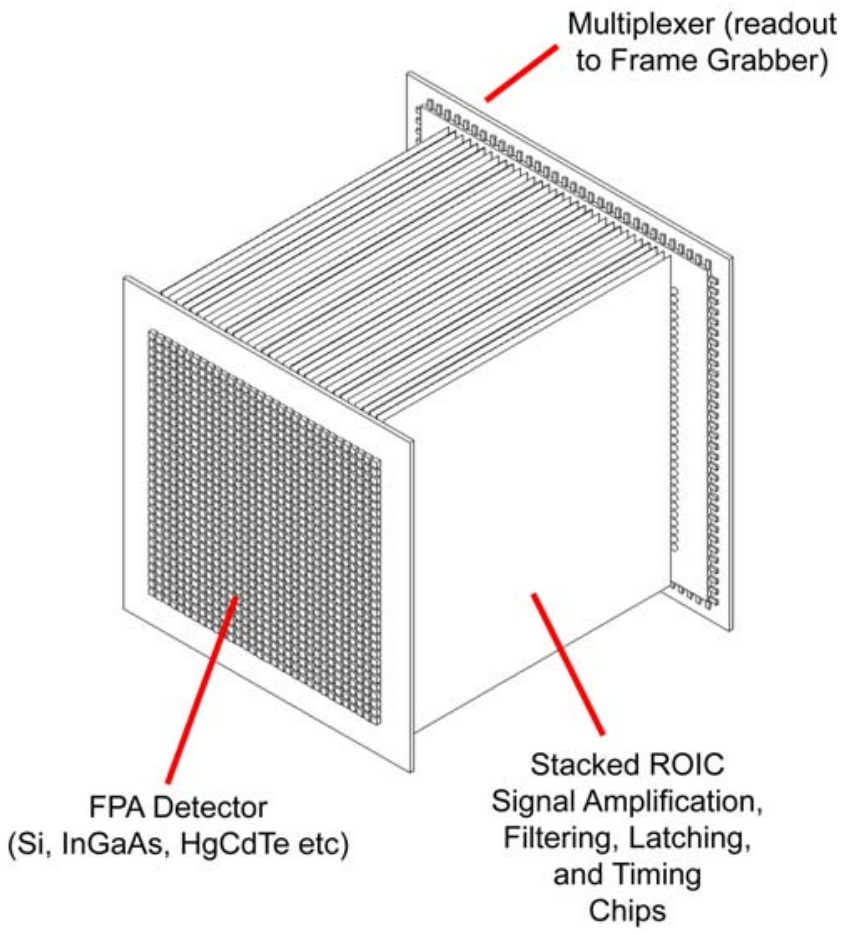

Figure 5.4.1: Z-stacked Detector Architecture. A planar FPA photon detector (photodiode or APD array) is back-side bump-bonded to a glued stack of thinned CMOS ROIC chips. Each ROIC chip handles one row of pixels from the FPA and the ROIC unit cells extend as pipellines down the length of the CMOS ROIC chip where they are then connected (through edge contacts) to a multiplexer chip. This approach provides a dramatic increase in unit cell circuitry real estate. 

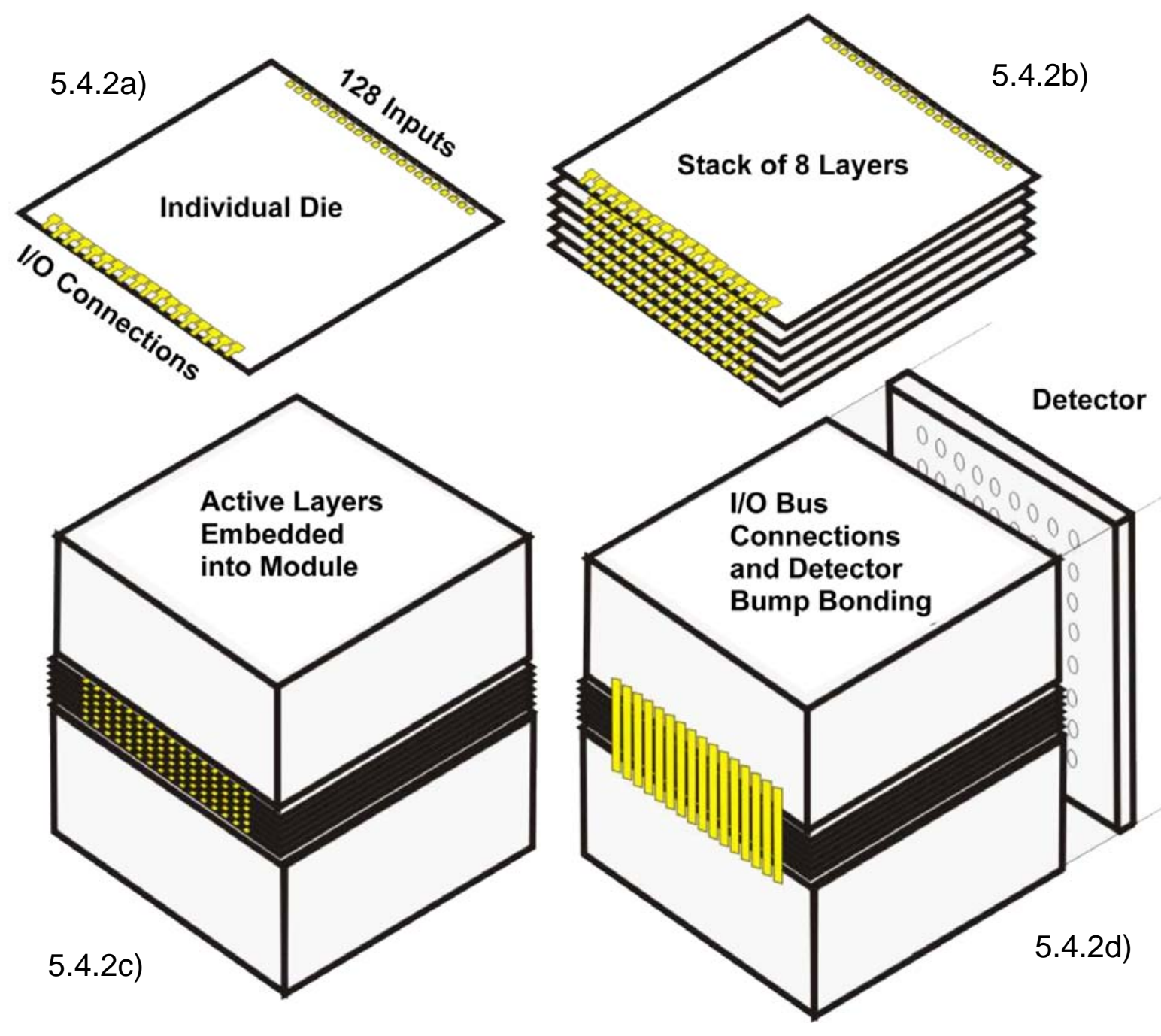

Figure 5.4.2: Chip Stacking in an FPA device. In the example shown above a $128 \times 128$ pixel detector array is to be timed by a stacked ROIC. a) Each ROIC die (layer) handles one row of 128 pixels. I/O connections are established at the ends of the thinned CMOS ROIC chips, usually through the use of indium bump bonds. b) The individual layers are aligned and glued (generally with a 1 micron glue line thickness) and edge bond contact pads are established on the faces of the composite slab. c) For test articles an 8-stack layer was bonded to dummy components to form a cube-like module. d) The FPA detector is then bump-bonded perpendicular to the plane of the stacked ROIC. Likewise, on the bottom of the stack, an I/O multiplexor chip is bonded on which serves as the conduit for the data to a frame grabber. Courtesy Irvine Sensors.

perpendicular to the plane of the detector, as shown in Figures 5.4.1, 5.4.2, and 5.4.3.

One can envision this as a stack of pancakes (note that this is only one several possible method of stacking). The signals run down strips and terminate in contact bumps at the edges of the ROIC chips and the FPA detector chip is bump bonded to the top 


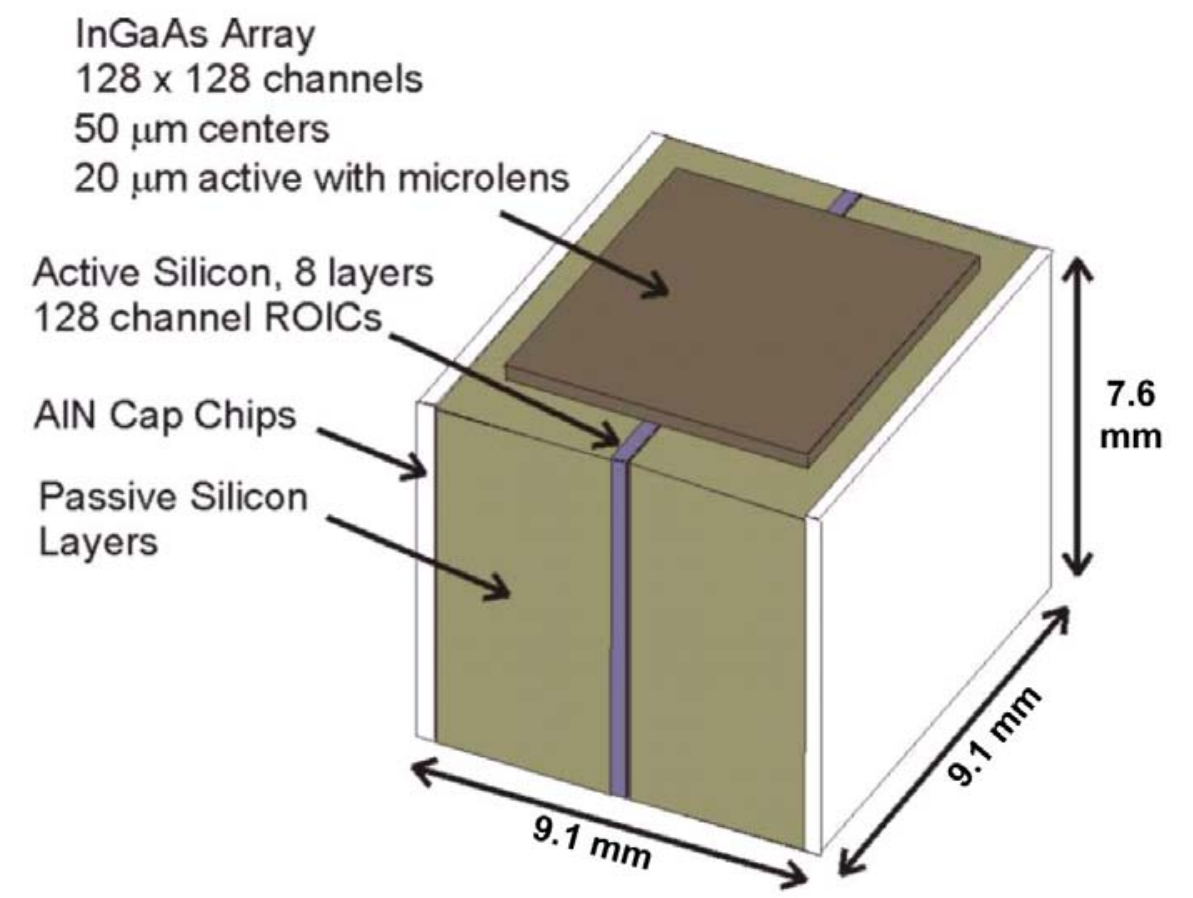

a)

Figure 5.4.3: Actual stacked ROIC and FPA sensor developed for captive flight tests. a) overall components definition and dimensions; b) top view showing APD detector chip; c) bottom view showing frame grabber interface contacts. Although originally conceived as a $128 \times 128$ active array, the test article included only eight ROIC active wafers, bump-bonded to eight rows of the overlying APD array. The remaining layers consisted of passive silicon. In tests the active $8 \times 128$ pixel array was scanned to achieve the desired sensor FOV. The majority of the depth of the sensor is devoted to storage of a 76 $\mathrm{m}$ long return signal record with a $75 \mathrm{~mm}$ bin size. (Courtesy Irvine Sensors).
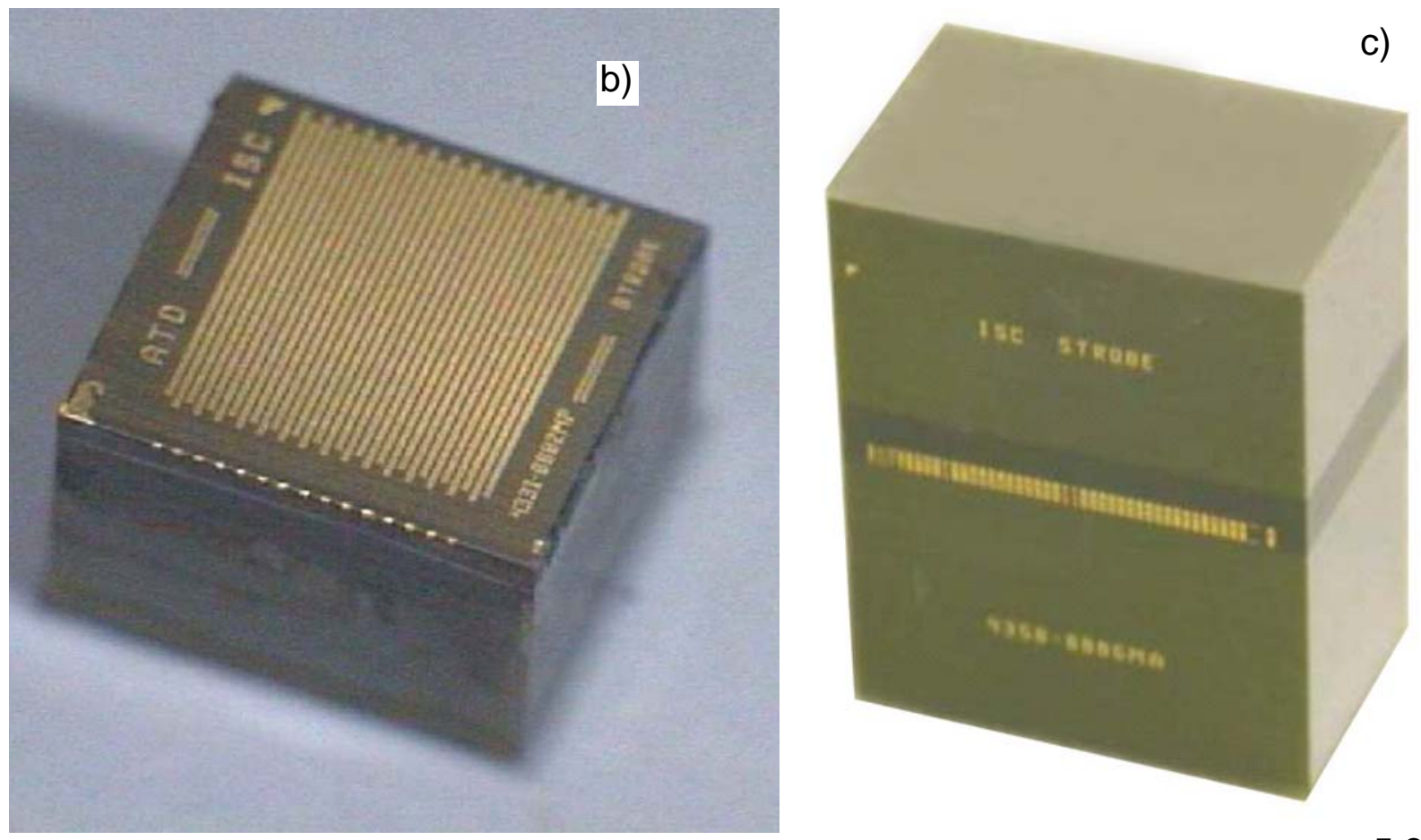
(see Figures 5.4.3 and 5.4.4). In this scenario, if one were developing, say, a 256x256 FPA, and the pixel pitch was 30 microns, then each ROIC stack slice (chip) would have to be on the order of $25 \mu \mathrm{m}$ to $28 \mu \mathrm{m}$ (allowing for inter-chip glue thickness) in order to allow for alignment with the next detector pixel row on the FPA. Each ROIC chip would then carry 256 unit cell pipelines (channels).

Current technology as a result of the DARPA JIGSAW program has seen the development of $30 \mu \mathrm{m}$ wide channels (unit cells) in the ROIC chip with capabilities for those unit cells to be up to $60 \mathrm{~mm}$ in length. Because such ROIC layers are CMOS devices it is possible to generate them economically through MOSIS on a wafer by wafer basis. The procedure is to mechanically grind the individual chips to $50 \mu \mathrm{m}$ thickness and then to chemically polish to $30 \mu \mathrm{m}$. The wafers can be diced part way through before lapping and will then come off the thinning wheel already diced without the requirement of handling a thinned wafer. Power dissipation and reduction is a major issue with stacked devices and special stack end caps of materials such as aluminum nitride or diamond have been used in such applications. Power reduction methods would involve the use of custom ASICs as opposed to COTS processors. It is generally believed that $512 \times 512$ pixel stacked arrays are feasible, although the largest that has thus far been produced in a working prototype is an $8 \times 128$ pixel array [Ludwig et al, 2004]. When gluing IC layers there is typically a $1 \mu \mathrm{m}$ glue line thickness. The procedure for connection of the assembled ROIC stack to the FPA is generally as follows:

etch silicon

passivate silicon

deposit contact pads

bump bond to back side of FPA

The location and sizing of the contact pads must be such as to allow for variance in spacing due to irregularities in the glue line thickness as well as mask tolerances on the FPA, and planarity, rotational and translational errors in the positioning of the various ROIC layers during gluing and setup.

Ludwig et al [2004] describe the first stacked LADAR ROIC:

The STROBE receiver is comprised of an innovative, stacked readout electronics module (ROIC module), support circuit cards, input/output interface connectors, 187 cubic inch housing and $67 \mathrm{~mm}$, F\# 1 optical system. It is a self-contained unit requiring only power and a $T_{0}$ (time zero) input to activate its time-of-flight operating system. Other command parameters include a prefire signal (for power dissipation management), a three-bit hold off word (for acquiring return signals from a distance greater than its storage depth), and a four bit threshold word (for threshold to noise ratio adjustment). 

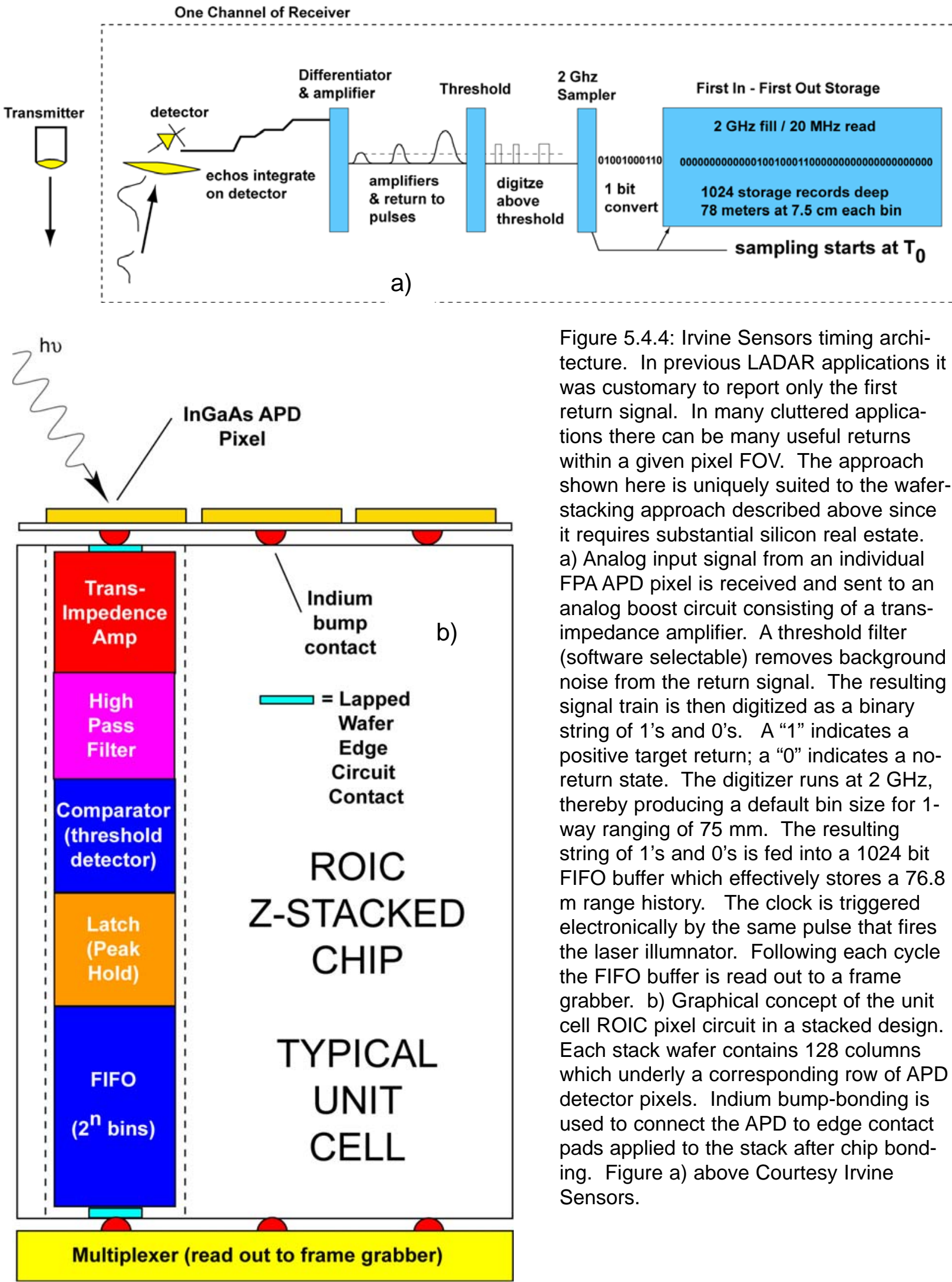

Figure 5.4.4: Irvine Sensors timing architecture. In previous LADAR applications it was customary to report only the first return signal. In many cluttered applications there can be many useful returns within a given pixel FOV. The approach shown here is uniquely suited to the waferstacking approach described above since it requires substantial silicon real estate. a) Analog input signal from an individual FPA APD pixel is received and sent to an analog boost circuit consisting of a transimpedance amplifier. A threshold filter (software selectable) removes background noise from the return signal. The resulting signal train is then digitized as a binary string of 1's and 0's. A "1" indicates a positive target return; a "0" indicates a noreturn state. The digitizer runs at $2 \mathrm{GHz}$, thereby producing a default bin size for 1 way ranging of $75 \mathrm{~mm}$. The resulting string of 1's and 0's is fed into a 1024 bit FIFO buffer which effectively stores a 76.8 $\mathrm{m}$ range history. The clock is triggered electronically by the same pulse that fires the laser illumnator. Following each cycle the FIFO buffer is read out to a frame grabber. b) Graphical concept of the unit cell ROIC pixel circuit in a stacked design. Each stack wafer contains 128 columns which underly a corresponding row of APD detector pixels. Indium bump-bonding is used to connect the APD to edge contact pads applied to the stack after chip bonding. Figure a) above Courtesy Irvine Sensors. 

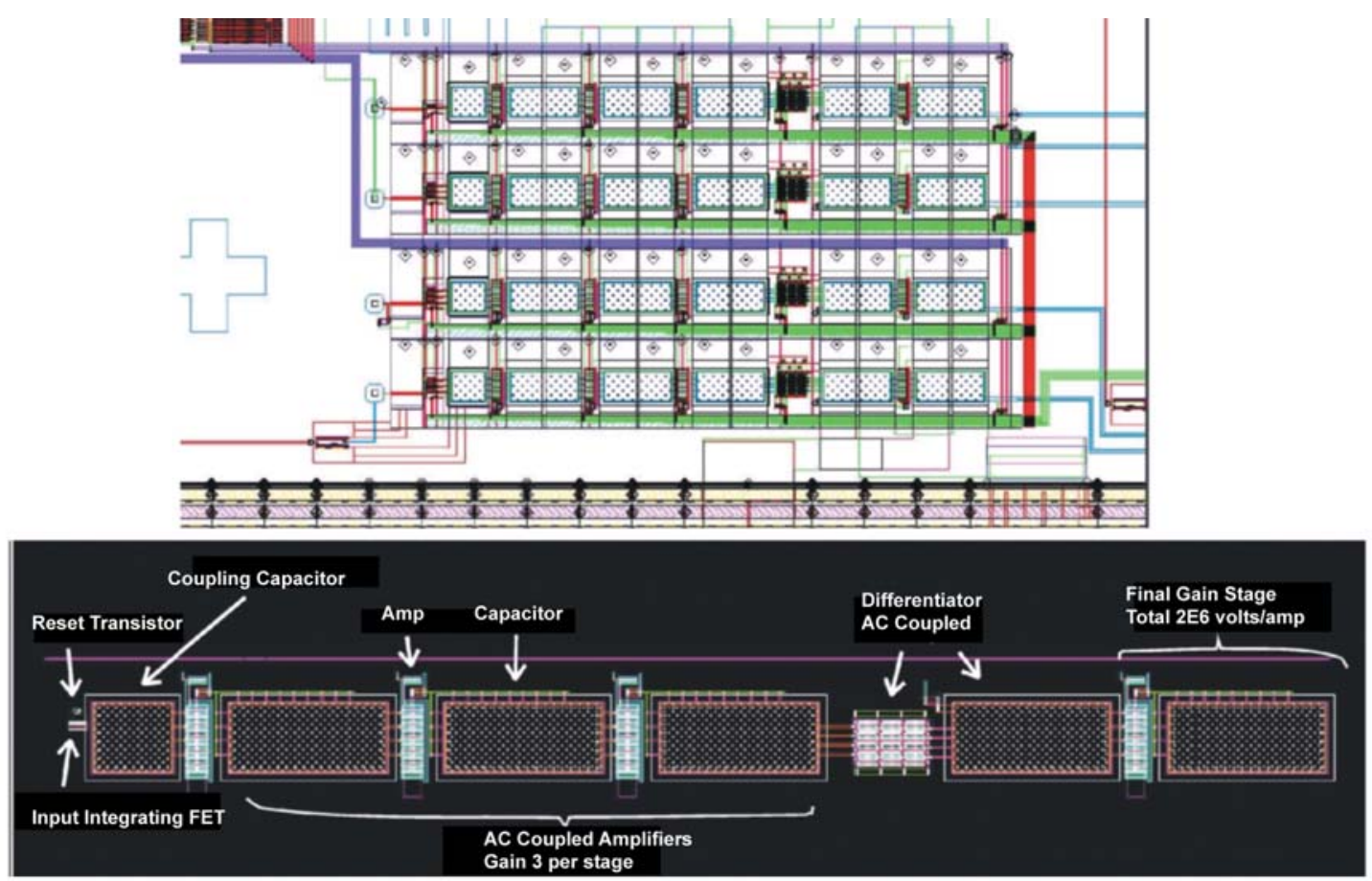

Figure 5.4.5: Photomicrograph showing a trans-impedance amplifier test article for the STROBE ROIC unit cell. The pipeline width is on the order of $30 \mu \mathrm{m}$ while the length can be up to $60 \mathrm{~mm}$. These dimensions are controlled predominately by current CMOS fabrication and chip stacking limits.

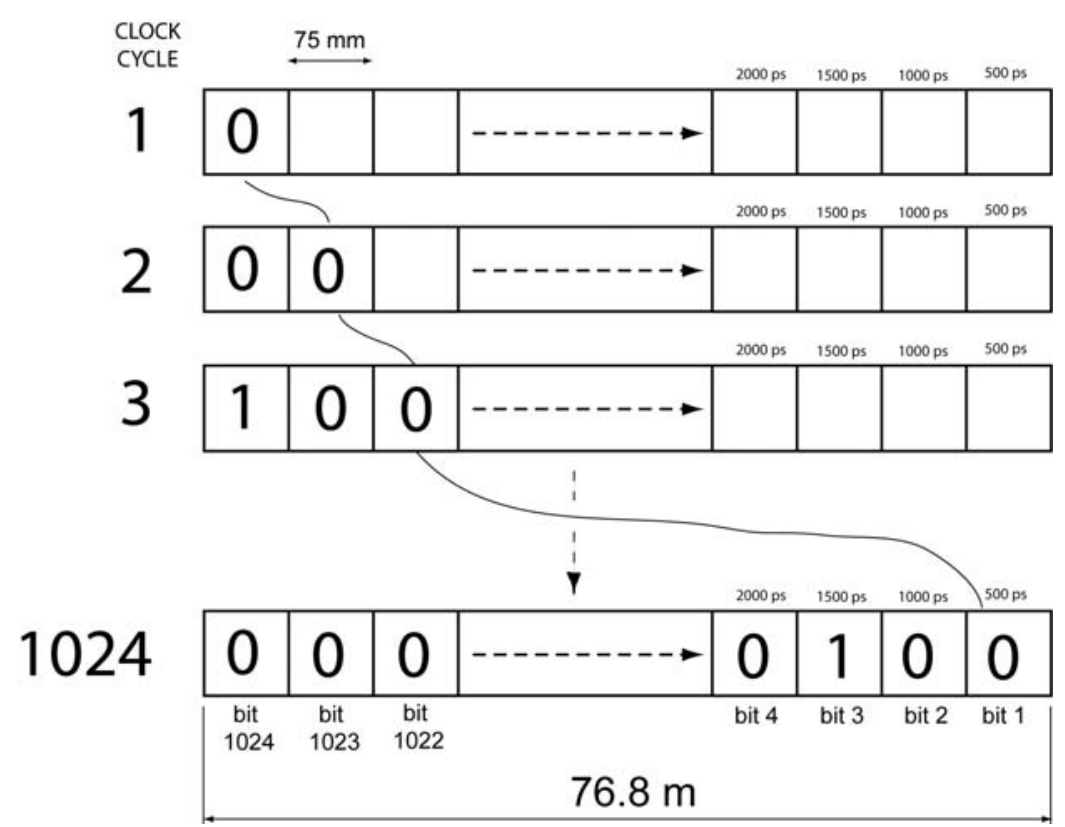

Figure 5.4.6: FIFO concept of storing time. Following signal amplification, filtering, and digitizing, the resulting return signal is stored sequentially as a string of 1's and 0's in a FIFO buffer. The speed of the digitizer determines the bin size (in this case a $2 \mathrm{GHz}$ clock yields a $75 \mathrm{~mm}$ bin), but the length of the FIFO determines the unambiguous range limit before aliasing. 

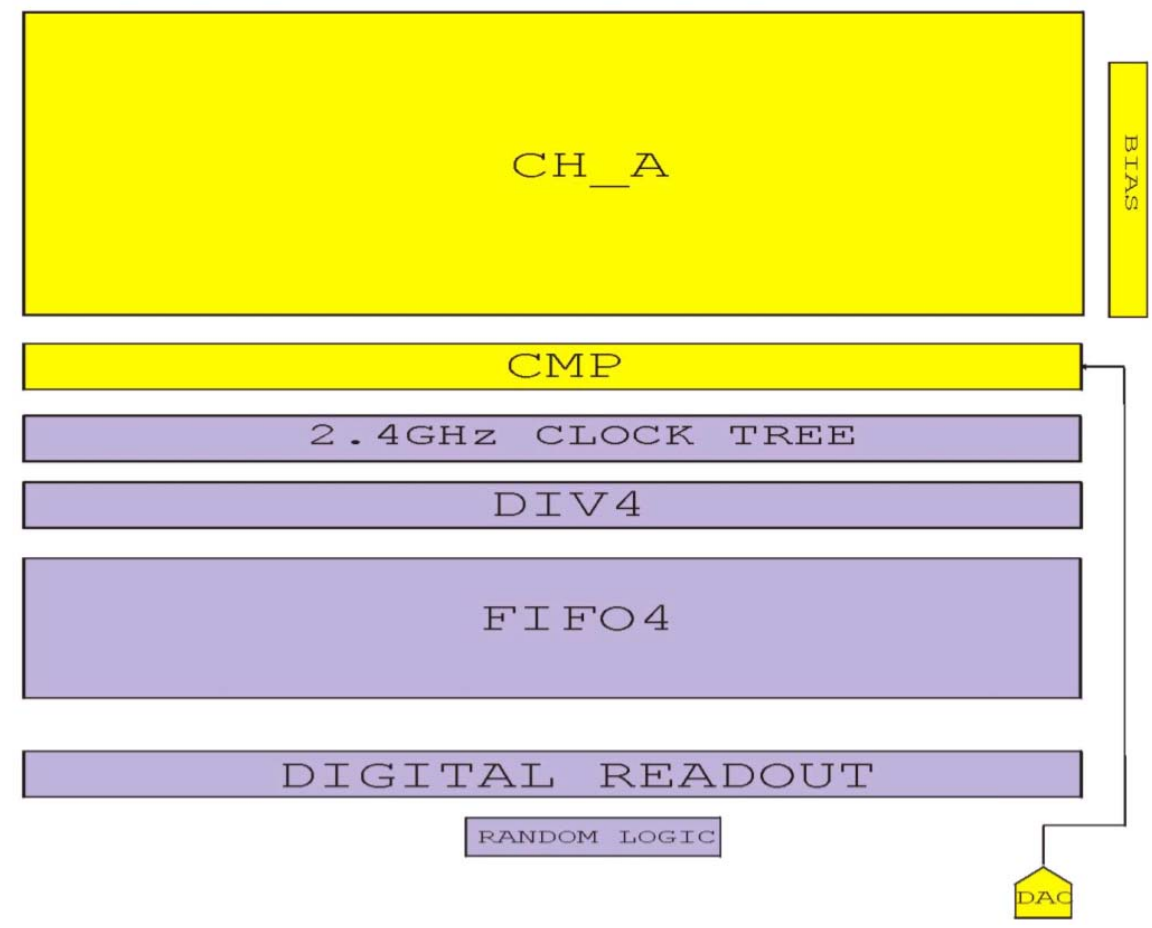

a)

Figure 5.4.7: a) ROIC Chip layout for timing a 128 pixel detector row; b) photomicrograph of the same chip. The large white section at the bottom, occupying more than $30 \%$ of the total chip area, is the 1024 bit FIFO buffer for storing the complete return signal. The chip can be considered as a collection of 128 vertical processing pipelines, each of which services an individual pixel from a particular row from the detector array.

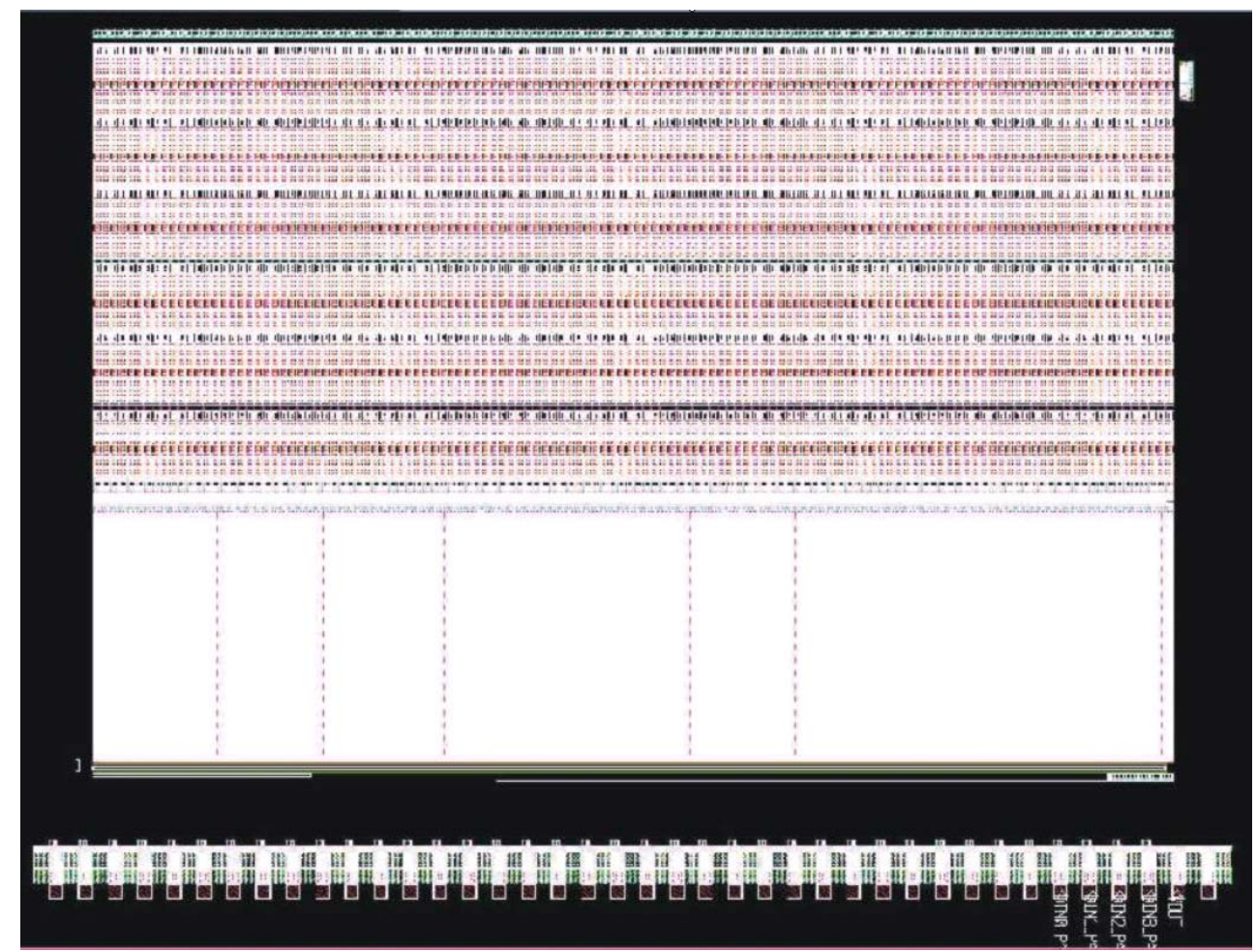

b) 


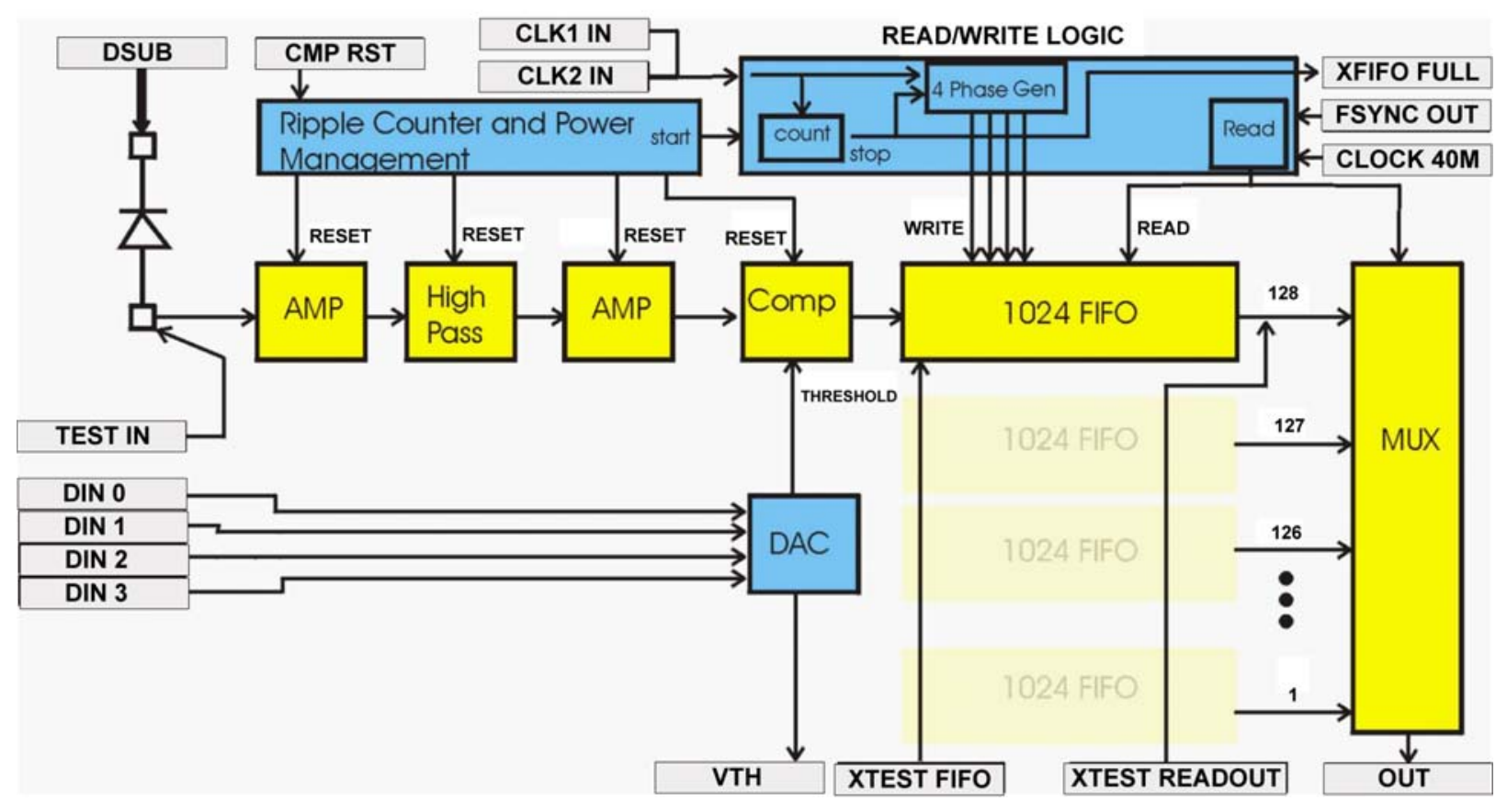

Figure 5.4.8: Block diagram of one signal processing channel for the STROBE ROIC. )Courtesy Irvine Sensors).

The heart of the receiver is a custom integrated circuit that counts time from the $T_{0}$ trigger to the time of the laser echo's arrival. Received laser echoes are integrated on the detector during a capture interval. The laser pulse is very short compared to the capture interval (500 ps versus $500 \mathrm{~ns}$ ) and multiple returns can be detected in a single pixel. The integrated signals are amplified and differentiated. This converts the signals back into pulses with an amplified signal strength great enough that they can be detected by a comparator (see Figure 5.4.4). The comparator detects if the reconstituted pulses are above or below threshold. The comparator's output is sampled at up to a $2 \mathrm{GHz}$ rate, resulting in a one bit $A$ to $\mathrm{D}$ converted signal. A 1024 deep FIFO (first in - first out) register stores a history of the comparator's output from $\mathrm{T}_{0}$ to $\mathrm{T}_{0}$ plus $500 \mathrm{~ns}$. With the FIFO clocking at $2 \mathrm{Ghz}$ each stage of the FIFO represents 500 ps of time history or equivalently $7.5 \mathrm{~cm}$ in range history. After the 1024 stages of the FIFO are filled, the ROIC begins to read the data out using conventional frame and line sync signals at a $20 \mathrm{MHz}$ rate. A high bit in the FIFO represents the time of arrival of a laser echo, determined by its location within the register.

A block diagram for a single channel of the ROIC is shown in Figure 5.4.8. Each ROIC contains 128 channels that are multiplexed to a single output. The STROBE focal plane is composed of 8 IC layers stacked to form a 128x8 pixel imaging array. The dies in the stack are thinned to $50 \mu \mathrm{m}$. The center-to-center spacing of the unit cells within an IC is also $50 \mu \mathrm{m}$, allowing a detector array to be bump bonded to the face of the module. The thinned active layers are imbedded into caps of sili- 
con to give the module height for handling. Input/output connection to the ROIC module is achieved by wire bonding from buses on the module (side opposite the detector) to a printed circuit board that attaches to the housing face assembly.

The approach described above involves straight-forward electronics and yields a

range resolution of $75 \mathrm{~mm}$. When considered on a pixel-by-pixel basis, the unit cell ROIC works in exactly the same fashion as first introduced in Figure 2.1.2, since this is a direct detection, or pulse time-of-flight architecture. The accuracy is dominated by the sample rate of the digitizer, which in this case is $2 \mathrm{GHz}$. This sets the sample bin size at $75 \mathrm{~mm}$. Setting aside issues of super-resolution techniques for obtaining subbin resolution, if one were interested in mm-level accuracy with such a sensor the digitizer would need to be running at approximately $150 \mathrm{GHz}$, thus generating $1 \mathrm{~mm}$ bins. For a measurement range of $100 \mathrm{~m}$ with $1 \mathrm{~mm}$ range resolution, a long FIFO is required. In Figure 5.4.7b we note that the chip area (height) devoted to the return signal storage FIFO is approximately $30 \%$ of the overall height of the wafer. Figure $5.4 .3 \mathrm{a}$ reports the wafer physical height as $7.6 \mathrm{~mm}$. This provides us with a rough estimate of $0.0022 \mathrm{~mm} / \mathrm{FIFO}$ logic element on the wafer. Figure 5.4 .9 provides an

approximate relationship between unambiguous range and range accuracy, using $60 \mathrm{~mm}^{*}$ as the

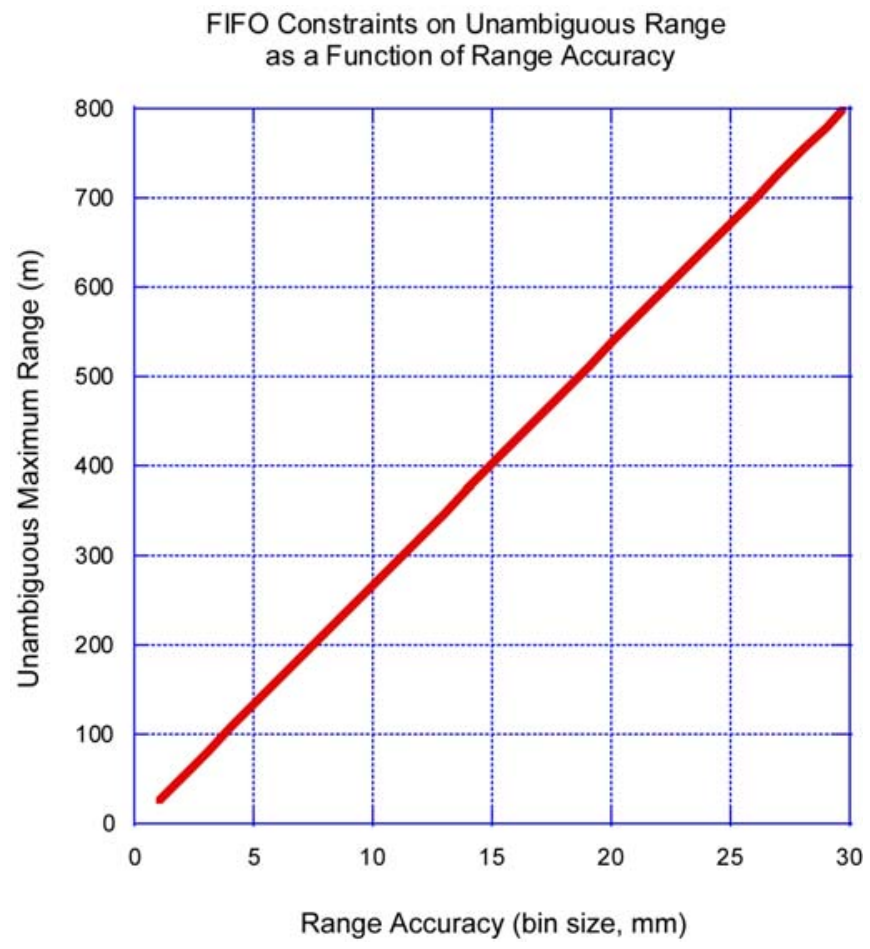
maximum usable wafer height (disregarding for the moment the real estate required for such things as analog trans-impedance amplifier lines, filters, latches, and digitizers). These figures indicate that a full response history per pixel could be stored and read out to frame grabbers at approxmately 4 $\mathrm{mm}$ bin size for a $100 \mathrm{~m}$ range. Commensurately smaller bin sizes yield the ability to store $1 \mathrm{~mm}$ accuracy at $25 \mathrm{~m}$ range. These all presume the availability of onchip digitizers (timers) operating at 37 and $150 \mathrm{GHz}$, respectively.

Figure 5.4.9: Unambiguous Range (in meters) for various bin sizes $(\mathrm{mm})$ assuming full CMOSwafer utilization for FIFO real estate in a pulse time-of-flight timing ROIC.

* $60 \mathrm{~mm}$ is considered the maximum currently achievable chip dimension in silicon CMOS fab technology (Dave Ludwig, Irvine Sensors, 2003 personal comm.) 


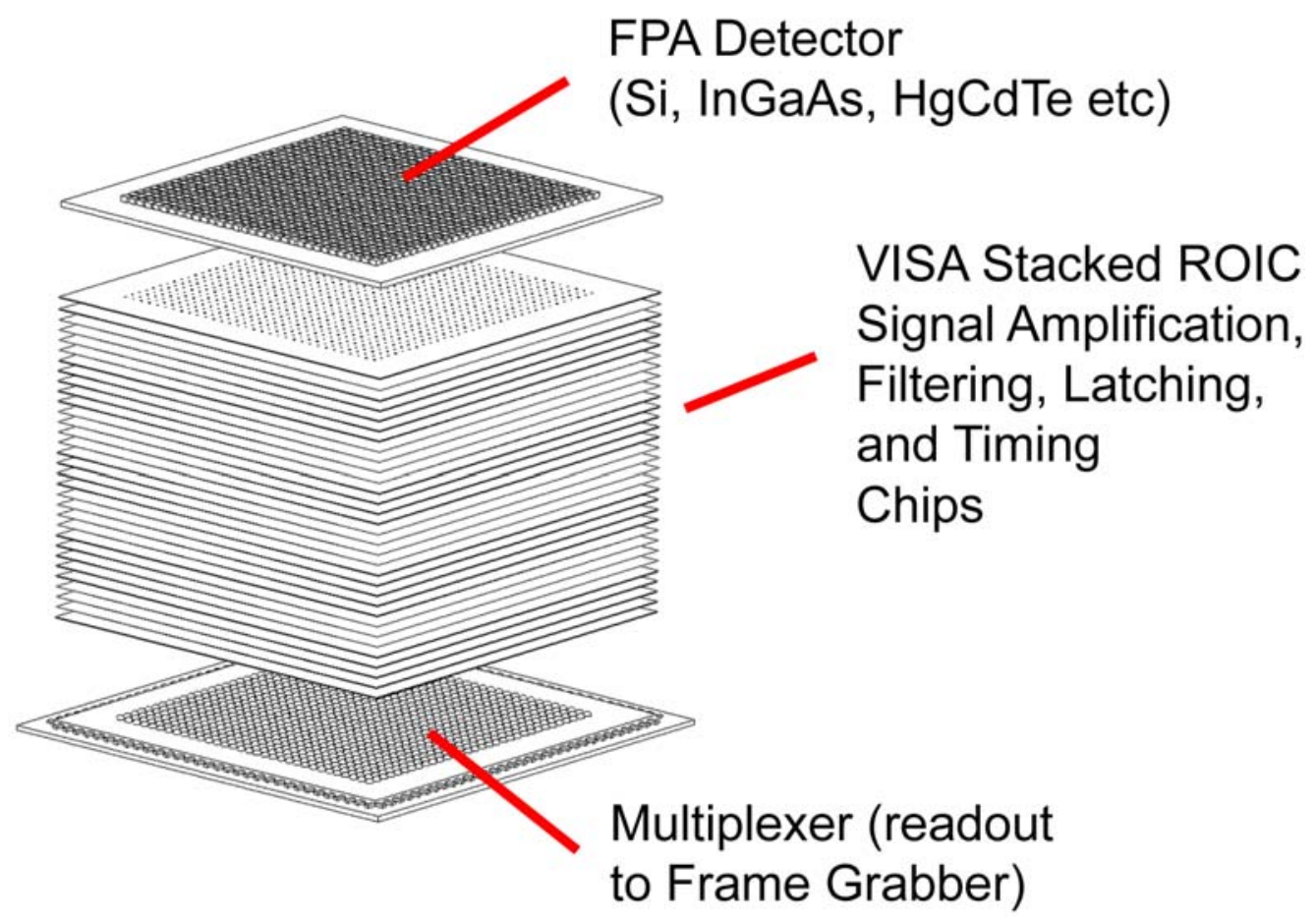

Figure 5.4.10: VISA stacking approach

\subsection{VISA Chip Research}

An alternate method of wafer stacking that is presently the subject of considerable research involves the use of horizontally stacked ROIC chips (Figure 5.4.10) and the use of vertical, thru-wafer vias, analogous to the concepts used for multi-layer printed circuitry board interconnects. The concept is known as Vertically Interconnected Sensor Arrays (VISA). Work in this area has only recently begun and there are no public-domain reports yet available on the results although it is anticipated that such information will begin to appear in 2005. DARPA describes the program as follows:

The program objectives are to develop and demonstrate vertically interconnected, FPA

readout technology capable of more than 20 bits of dynamic range at array readout rates exceeding $10,000 \mathrm{~Hz}$ in 3D unit cells with pixel footprint areas of smaller than $25 \mu \mathrm{m} \times 25 \mu \mathrm{m}$. The dynamic range of an FPA is defined to be the ratio of the highest signal power which can be linearly measured in an illuminated pixel to the equivalent noise power measured in an un-illuminated pixel operating under the same readout conditions. The extremely wide dynamic range can be accomplished by novel multilayer readout circuits vertically connected to individual detectors in stacked single-or multi-color 2D staring arrays. These circuits may enable imaging at more than 20 bits of dynamic range, where the current state of the art (around 10 bits) is over an order of magnitude lower. The ability to bring signals directly from the detectors to the readouts in a highly parallel manner without initially going through row-column multiplexers will allow for high frame rates concurrently with 
high-resolution images.

Specific interest is in the development and implementation of massively parallel, densely interconnected imaging focal plane architectures. This is expected to result in the achievement (separately or in concert) of high resolution, high fill-factor pixels, ultra-wide dynamic range (greater than 20 bits), multispectral, and very fast imaging capabilities. [The program is focused on] (1) Process technology for dense interconnects (electrical or optical) through three-dimensional structures including via generation, wafer bonding, and thermal management. The ability to demount and repair stacks may be important; (2) Novel circuitry that capitalizes on direct connection to the detectors so as to achieve adaptive signal processing heretofore impossible.

If achieved, such approaches could lead to dramatic cost and size reductions for

FPA sensors. One advantage of the VISA approach is that, if proven successful, it will effectively avoid the unit cell length restriction $(60 \mathrm{~mm})$ implicit in CMOS foundry wafer production. On the other hand, practical limitations of bonding quality control and alignment may limit the effective number of layers. Irvine Sensors has posited that a $512 \times 512$ pixel Z-stacked FPA is feasible but beyond that heat dissipation and manufacturing quality control become serious problems. Taking this as a basis, Figure 5.4.11 (below) suggests that a Z-stacking approach yields upwards of $5 \mathrm{X}$ more circuitry real
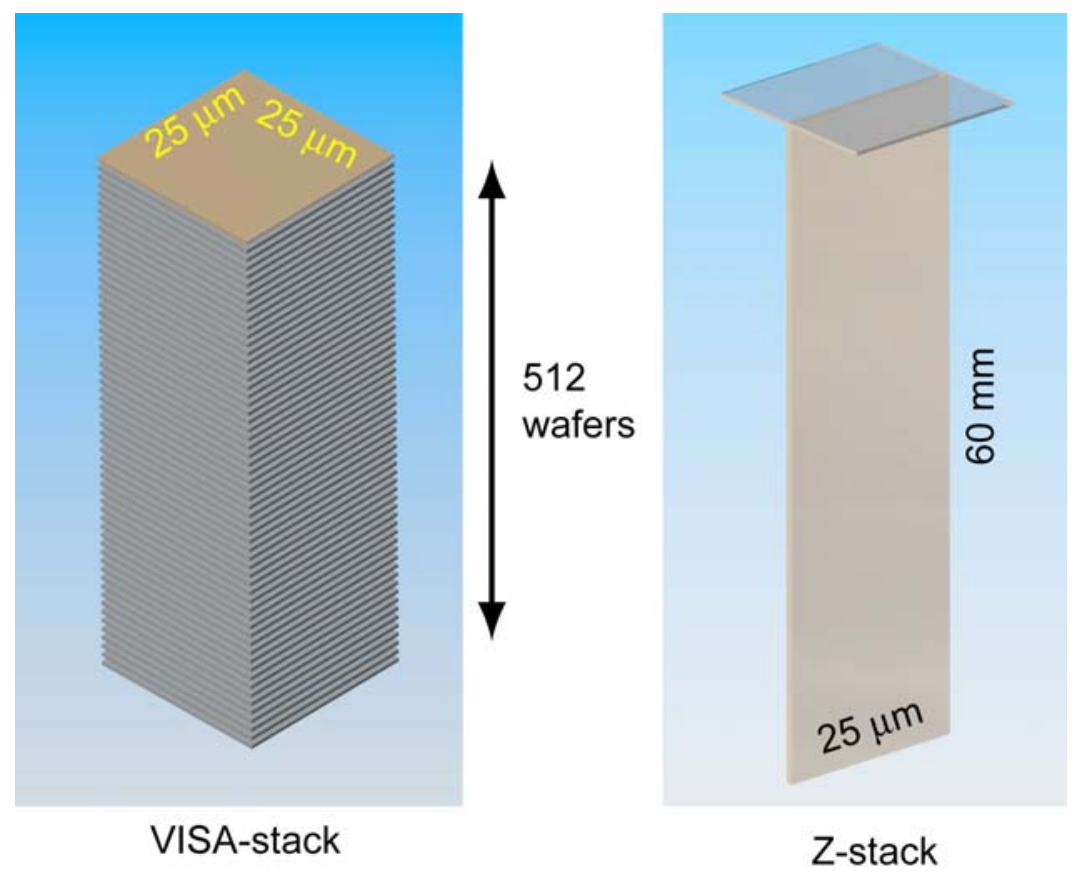

Figure 5.4.11: Anticipated physical limits of VISA and Z-stack time-of-flight ROICs. 
estate per unit cell than the VISA approach. This may not be a factor in low resolution real-time 3D cameras, but high resolution systems will push these limits.

\section{6 $\underline{\text { Coherent Solutions }}$}

Discussion in this chapter has thus far dealt with pulse time-of-flight or AM homodyne timing concepts. It is feasible to construct coherent FPAs ${ }^{1}$, but no one has yet done so. There are significant design requirements that must be resolved in addition to all of the issues described above. For example, each spatial pixel must have a diffraction limited field of view, in order for it to be an optimally efficient optical heterodyne receiver. In a coherent FPA the multipixel scene would be imaged onto the coherent ladar focal plane and each pixel would present lambda/(aperture diameter) in angular extent and image a distinct portion of the scene. Then, one optical local oscillator beam could be broadcast to all of the pixels either by freespace or guided wave optics in order to optically mix with the signal echoes on each of the pixels. Less than a milliwatt of LO would be needed on each pixel for heterodyne detection (no LO is required for autodyne detection) so the LO power required would be modest for up to a 10x10 array which could subsequently be scanned.

\footnotetext{
1 Personal communication Duane Smith, Coherent Technologies, Inc.
} 


\subsection{Status Report on NIST BAA: Next Generation LADAR for Driving Unmanned Ground Vehicles}

\subsection{Background}

The U.S. Department of Defense has initiated plans for the deployment of autonomous robotic vehicles in various tactical operations starting in about 7 years. Several programs, including the Future Combat Systems program, have received significant funding in order for this to take place. Envisioned are manned and autonomous unmanned ground vehicles as well as manned and unmanned air vehicles performing cooperative tactical missions. Some of the tactical missions being considered for unmanned ground vehicles include: Reconnaissance, active or passive surveillance, communication relay, mine detection or clearing, targeting, search and rescue, supply, terrain control/denial, forward observation, and lethal or non-lethal missions. These missions will require the vehicles to drive autonomously over open terrain and on roads which may contain traffic, obstacles, military personnel as well as pedestrians. UGVs must therefore be able to detect, recognize and track objects and terrain features in very cluttered environments.

Although several LADAR sensors exist today which have successfully been implemented and demonstrated to provide somewhat reliable obstacle detection which can be used for path planning and path selection, they tend to be limited in performance (primarily resolution and maximum range), are effected by obscurants (dust, fog, grass, foliage), are quite large and expensive. An example of where the current technology falls short is shown in Figure 6.1. Although the flatness of the road is visible in the display of the 3D range image of the road the vehicle is following, it has very limited range information from the road and terrain further ahead in the scene.

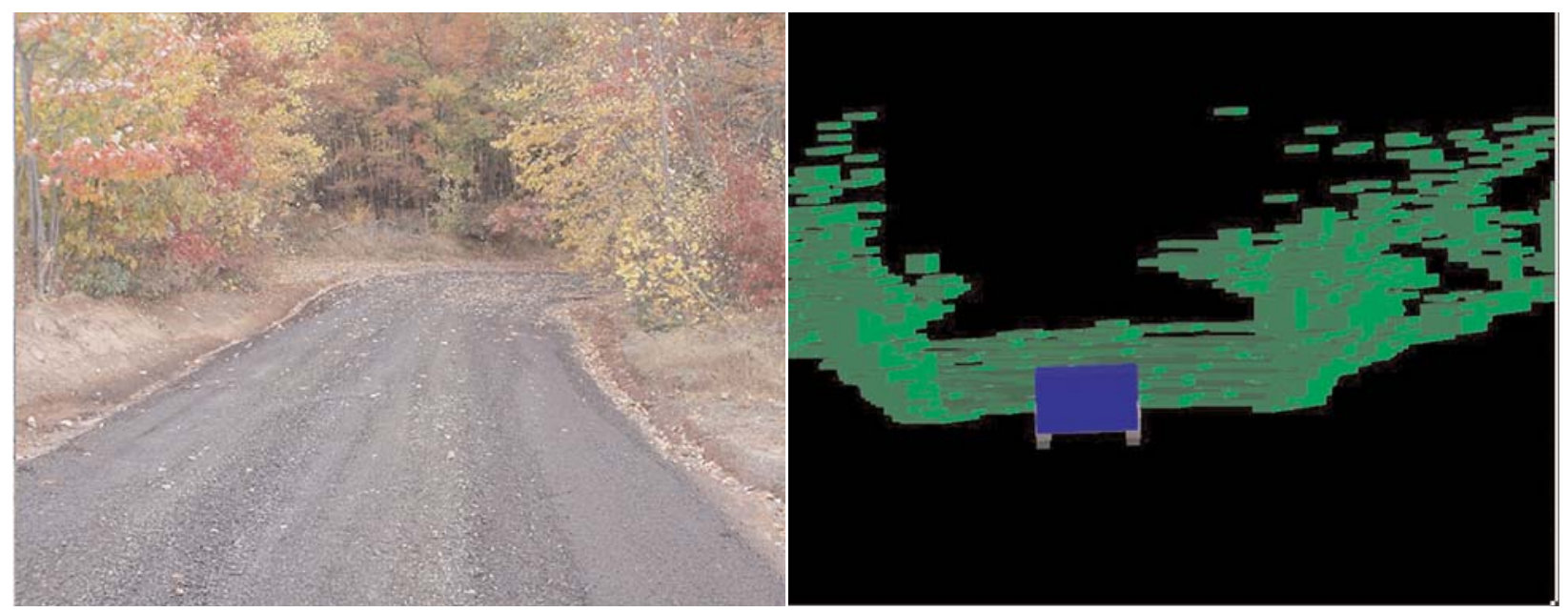

Figure 6.1: (left): Digital camera image of road and trees; (right) the same scene as viewed by real-time (GDRS) LADAR. Where does the technology fall short? The vehicle is effectively myopic. Current LADAR is low resolution (75 $\mathrm{mm}$ to $150 \mathrm{~mm}$ accuracy) and short range $(50 \mathrm{~m})$. Current LADAR does not penetrate dust and smoke. Planning is not optimized for road following. 
In addition, although the trees and brush along the sides of the road are seen as obstructions, it would be very hard to use that information for classifying or identifying what those obstructions are. Another example of current technology limitations is shown in Figure 6.2. The figure displays the range image of a scene taken in a parking lot. It is very hard to identify any detected known objects in the scene. In the future, it is expected that unmanned vehicles be able to locate available parking spaces on their own and to be able to park autonomously.

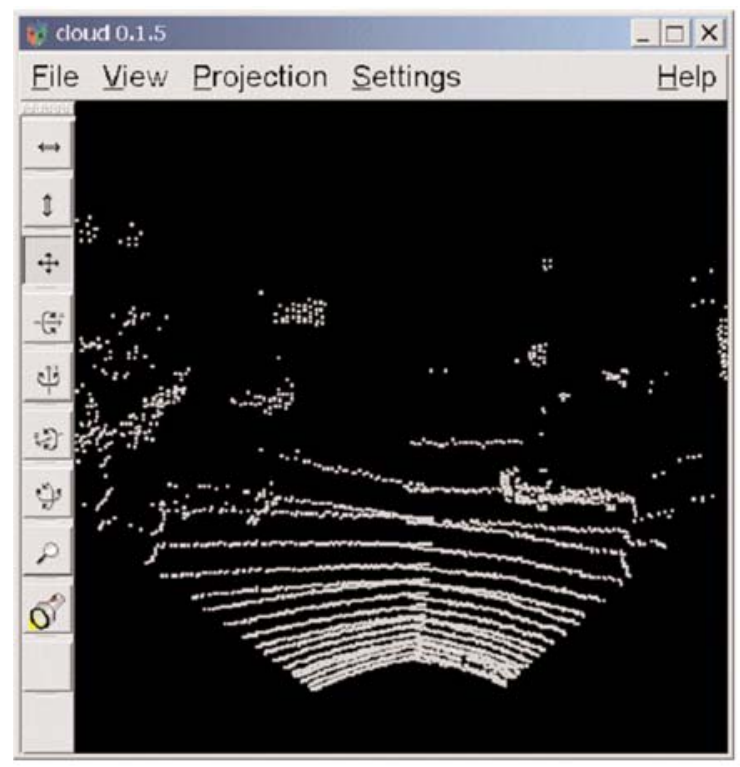

Figure 6.2: Typical point cloud produced by a coarse UGV LADAR.

What denser, high resolution range data can provide is clearly evident in the range image taken of a parking lot with a high resolution LADAR camera. This is shown in Figure 6.3

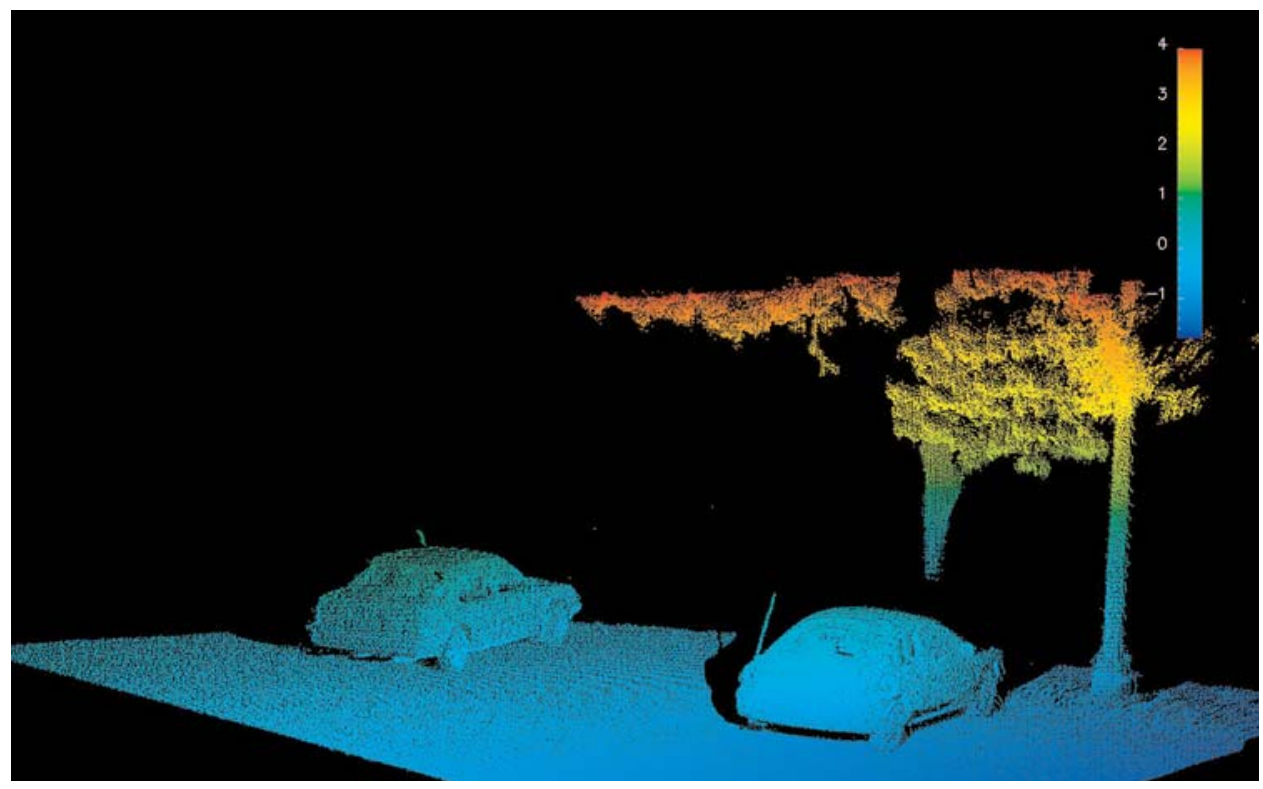

Figure 6.3: High resolution point cloud image of parking lot. Image is color-coded in vertical (z) dimension. 
Detection and identification of cars, tree trunks, overhanging branches, man-made structures (light pole) and even curbs are highly possible from this kind of data. It must be pointed out, however, that the data was taken with a high resolution scanning LADAR that required several minutes to generate the range image.

\subsection{BAA Status Report}

Even though considerable effort and funding has been provided by the DOD R\&D community, nearly all of the development has been for target detection (ATR) and tracking from various flying platforms. This includes LADAR technology development which allows for foliage penetration, permitting detection of target hidden under trees and camouflage netting. Although much of the development has contributed significantly for furthering the performance of LADAR sensors, they have not addressed the needs for autonomous driving with unmanned ground vehicles. LADAR sensors for ground vehicles have their own particular requirements. This includes having a very broad dynamic range. This is the ability of the sensor to detect and recognize obstacles/objects/terrain features which are at very close range $(<1 \mathrm{~m})$ and at more than $100 \mathrm{~m}$, all in a single frame of data.

Participation in the Army Demo III program, has helped NIST to identify requirement specifications for LADAR to be used for on and off-road autonomous driving. NIST envisions the need for two types of LADAR range imaging sensors for this type of application. One having a wide field-of-view $\left(40^{\circ} \times 9^{\circ}\right)$ with a resolution of about .25 degrees or better per pixel and the second, a foveal LADAR having a narrow field-ofview of approximately $1 / 10$ th of the wide field-of-view with a resolution of about .05 degrees or better per pixel. The intent is to make the foveal LADAR quickly steerable to (points-of-interest) positions within the wide peripheral angle field-of-view LADAR at a rate of at least 3 saccades per second. Both types of LADAR sensors should have a resolution of about $5 \mathrm{~cm}$, or better, in range, shall be able to detect the ground plane out to a distance of about $40 \mathrm{~m}$ and vertical surfaces out to a range of at least $100 \mathrm{~m}$. Frame rates of higher than $10 \mathrm{~Hz}$ are expected. Both LADAR must be eye safe and shall be provided with the capability to penetrate dust, fog, grass and light foliage (either by sensing multiple returns or looking for the last return), and be able to operate in full sunlight conditions. Small size and low cost were also emphasized as important requirements.

The initial BAA announcement, which contained details on the expected requirements, was released in June of 2002 and is attached as Appendix B for reference. There was a good industry response to the announcement with 15 proposals being submitted. A unanimous decision was made by the proposal reviewers to make four awards for Phase I at the end of September. The four awards went to (listed in alphabetical order): 
- Advanced Scientific Concepts Inc., Santa Barbara, California

- Coherent Technology Inc., Lafayette, Colorado

- Lockheed Martin Missiles and Fire Control, Dallas, Texas

- Raytheon Missile Systems, Tucson, Arizona

Synopses of some of the main unique features of each design was prepared by each contractor for inclusion in this report. These are provided in Section 6.3. Because of the proprietary nature of the designs, however, the contractors desired to keep much of the information confidential until an award is made for follow-on development.

At the time of this report, the Phase I LADAR designs have been reviewed, but, plans for the next phase of development are on hold pending availability of funds. Requests for additional information can be directed to the contact person or persons listed at the end of each synopsis.

\subsection{Synopses of Designs}

The following are the brief technical synopses offered by the four Phase I BAA winners on their proposed designs for a next generation LADAR for Driving Unmanned Ground Vehicles.

\subsubsection{Advanced Scientific Concepts Inc. (ASC)}

\section{Compact Unmanned Ground Vehicle LADAR (CUGVEL)}

The objective of the Advanced Scientific Concepts Inc.'s (ASC) contract effort was the design of a 3-D Flash LADAR system that met specifications discussed elsewhere in this report. The ASC system is called the Compact Unmanned Ground Vehicle LADAR (CUGVEL). The ASC designs used COTs parts as much as possible to reduce cost. In addition a very compact laser was used to reduce volume, weight and power. Two alternate WFOV designs are discussed using two different photon-amplification detector-array systems. In the first of these designs there are no mechanically moving parts and only a single laser pulse is needed to capture the entire WFOV. This system is called the single-pulse WFOV system and uses an image-tube amplification system. In the second WFOV design two laser pulses are required to capture the entire WFOV and there is some minor, two-position, mechanical motion to achieve the full field of view. This system is called the two-pulse WFOV system and uses APDs for the detector system. APDs provide less photon amplification than image tubes.

Both WFOV systems work well as stand-alones. A gimbal-based foveal system with longer focal length optics than the WFOV systems, but with the same 3-D FPA, that can access the full WFOV appears to be completely adequate as a NFOV stand-alone. There are advantages for having two WFOV and NFOV imaging systems; objects that require fine resolution can be investigated completely independently, without affecting the WFOV frame rate. In addition the optics is simpler. The disadvantage is the increased weight and volume of two separate systems. 
A combined WFOV and NFOV system is also possible. When combined with a foveal system the ASC WFOV systems offer a simple design. However, the combined systems put increased acceleration requirements on the gimbal in order to satisfy the WFOV frame rate specifications. As currently designed with off-the-shelf optics, all the 3-D imaging systems are two aperture systems whether they are WFOV, NFOV or a combination of the two: an aperture for the laser-transmit optics and an aperture for the 3-D imaging, receive optics. Figure 1 illustrates the single-pulse WFOV design. Single aperture CUGVEL systems are possible at an increased cost with potentially reduced weight and volume. Other proprietary design features enhance CUGVEL object identification.

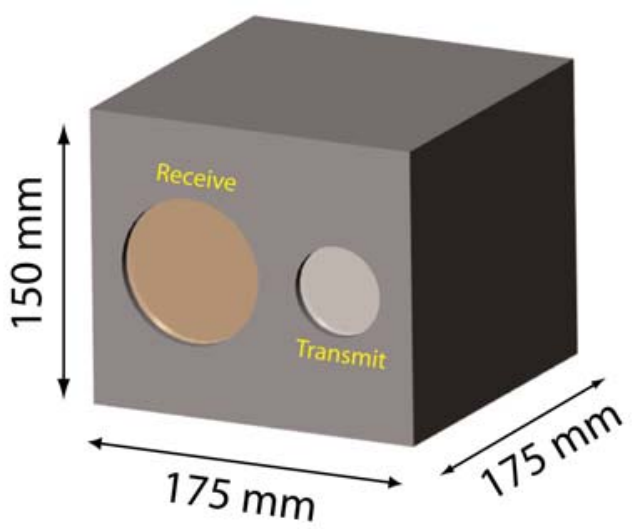

Figure 6.4: Approximate packaged configuration for the standalone WFOV CUGVEL. Approximate weight is 1.8 $\mathrm{kg}$; the COTs optics is a large fraction of this weight.

Additional information on focal plane array technology development is presented in Chapter 5. Additional information on the BAA Phase I designs for the CUGVEL can be requested from Roger Stettner (rstettner@advancedscientificconcepts.com) phone: 805-966-3331.

\subsubsection{Coherent Technologies Inc. (CTI)}

\subsubsection{Synopsis of the Architectural Approach}

$\mathrm{CTI}$ has designed an innovative, first-of-class coherent Frequency Modulated Continuous Wave (FM-CW) LADAR for an UnMANNED Ground Vehicle (UGV) 3-D imaging sensor. The proposed approach is a departure from conventional direct and coherent detection designs. It offers significant size, weight, power, and performance advantages over direct detection systems such as pulsed Time-of-Flight (TOF) or Amplitude-Modulated Continuous Wave (AM-CW) waveforms. It also offers cost reduction over conventional pulsed coherent detection designs. For the NIST-specified UGV sensor, the compact coherent FM-CW system meets or exceeds all narrow field-ofview (FOV) requirements (few $\mathrm{cm}$ range resolution, 200m range, IFOV $<1 \mathrm{mrad}$ $\left(0.05^{\circ}\right)$, frame rate $\sim 20 \mathrm{~Hz},>9^{\circ} \times 9^{\circ} \mathrm{FOV}$ ) and provides pan/tilt mosaics for the wide FOV $\left(40^{\circ} \times 90^{\circ}\right)$ operation with moderate frame rates. The narrow FOV high-resolution sensor meets the most stringent requirements while also providing higher sensitivity, 
superior countermeasure/jam/spoof/damage resistance, and greater range-scalability compared to alternative direct detection architectures.

\subsubsection{Advantages of the Approach}

- The innovative optical (not RF) homodyne receiver architecture offers a path to very high range resolution $(0.75 \mathrm{~cm}$ to $3 \mathrm{~cm})$ through high effective bandwidths $(10 \mathrm{GHz}$ to $40 \mathrm{GHz}$ ) that would be difficult or costly to achieve with electronics-based receivers in direct detection systems, whether they be pulsed TOF, AM-CW, or other variants.

- The unusual homodyne receiver reduces signal processing burdens by more than 10 times versus conventional broadband signal processing approaches. The innovative approach eliminates the need for range compression by de-ramping, or operating at full $40 \mathrm{GHz}$ class bandwidths.

- High quantum efficiency ( $80 \%$ ) shot noise-limited performance is obtained by the coherent transceiver where mixing function in the optical domain as opposed to the RF domain, where AM-CW transceivers operate. RF heterodyne receiver noise, while quite low, cannot match the ultra-quiet performance of optical heterodyne receivers. Because the sensitive transceiver operates at the fundamental limits of low operating power, it minimizes the probability triggering opponent's laser warning receivers.

- The transceiver aperture is a few millimeters in diameter vs. many centimeters required by most direct detection technologies for equivalent power-aperture products $(<0.1 \mathrm{~W} / \mathrm{cm} 2)$. The miniature aperture significantly simplifies the scanner design; thereby enabling high-speed raster scans with a compact low power system that would be difficult and costly with larger apertures. The smaller sensor aperture is also more defensible against kinetic energy weapons and more covert in battlefield environments by offering smaller optically augmented returns for adversary's anti-sensor searches. While a Geiger Mode Avalanche PhotoDiode (GAPD) receiver has comparable sensitivity to the proposed coherent transceiver, it has other limitations that make it undesirable (see below).

- The laser power is transmitted with $100 \%$ duty cycle, enabling full utilization of the energy to generate target returns and receiver signal. Extra-cavity amplitude modulation schemes are inherently less efficient due to rejected laser power and gain modulation brings many trades, including unwanted sidebands at high modulation depth. The FM-CW waveform does not suffer these disadvantages and CW operation allows optimization of the threshold, slope efficiency, and dynamic reserve of efficient $1.5 \mu \mathrm{m}$ diode lasers and higher power, scalable ion-doped waveguide lasers.

- Costly master oscillators were eliminated in the design, making a coherent transceiver practical for this moderate volume, cost-sensitive market. Cost was a critical driver in the system-level design. The innovative signal processing and concomitantly simpler MO were motivated by the need to reduce cost and complexity. 
- Highly linear frequency modulation is not required by innovative servo and sensor designs. The signal processing approach also does not require long range record processing. Multiple report range records are possible, along with maximum report, last report and "n largest" reports. Highly linear frequency sweep requirements have been a critical problem and nearly show-stoppers in other radar and LADAR system designs, so avoiding the challenge is valuable.

- Optically mixing the FM-CW waveform does not encounter the $3 \mathrm{~dB}$ SNR loss that is inherent in AM-CW transceivers (single sideband demodulation) and other RF domain mixing receivers.

- Countermeasure resistance is unmatched for the coherent transceiver. While a Geiger Mode Avalanched Photodiode (GAPD) receiver offers sensitivity matching that of a coherent transceiver, high quantum efficiency GAPDs do not yet exist at $1.5 \mathrm{~m}$ wavelength. If an acceptable $1.5 \mu \mathrm{m}$ GAPD existed, it would be highly susceptible to jamming and countermeasures. One such countermeasure would be corner cubes, with few mitigation approaches available because of GAPDs essentially "zero dynamic range" ( 1 bit only) and microsecond class reset/recovery time before it can detect the next photon. Operating existing GAPD transceivers from $750 \mathrm{~nm}$ to $1064 \mathrm{~nm}$ non-eyesafe wavelengths means that the system can be detected by advanced night vision gear, complicating covert operations. The proposed coherent transceiver can reject large in-spectral band jamming signals by Doppler filtering. Immunity to high intensity jamming is conferred by the fact that the local oscillator is already intentionally driving the receiver to the saturation limit of the detectors. Damage due to very high pulse energy countermeasures requires additional approaches such as nonlinear optical power limiters at the exit/entrance pupil of the transceiver.

- A coherent transceiver is inherently more immune to background interference. It can look into the sun's disk and maintain operation where this is difficult to do with any direct detection transceiver, especially GAPD-based designs. Again, the fact that the coherent local oscillator is putting milliwatt-class average power on the detectors means that background light is inconsequential. Still, some care must be exercised to choose narrow spectral band pass filters on the receiver.

- Coherent transceivers offer significantly higher dynamic range than direct detection transceivers. A coherent transceiver detects the field amplitude, not the intensity of an echo as direct detection transceivers do. CD receivers typically have an $80 \mathrm{~dB}$ dynamic range vs. $40 \mathrm{~dB}$ for $\mathrm{DD}$ systems because they are detecting the square root of the intensity. The dynamic range of GAPD receivers is especially low (dynamic range of $0 \mathrm{~dB}$ ) vs. other DD receivers because it cannot collect intensity data on a single echo and they must infer it from a statistically large number of samples. The wide dynamic range improves countermeasure resistance and the ability to detect last reports in difficult situations, such as seeing terrain dips and trip-wires through tall grass. 
- The proposed design utilizes small pixel count focal plane arrays, but can utilize large ones as they become available. Coherent detection focal plane arrays are in their infancy, as are direct detection FPAs. Coherent FPAs are being developed at CTI and other locations, but their bandwidth, record length, and pixel count leave something to be desired, thus far. Small linear coherent arrays can be produced at high bandwidth and therefore are the baseline for the proposed transceiver. Small pixel count arrays (1x8 for example) are also significantly less costly than larger arrays.

- Speckle is mitigated in the proposed high bandwidth ( $>4 \mathrm{GHz}$ ) transceiver. There are occasional concerns about the "grainy" character of coherent ladar images. This is not a driving issue here because first, the proposed sensor is only recovering range data, not intensity data, and second, the spectral diversity for a $4 \mathrm{GHz}$ to $40 \mathrm{GHz}$ bandwidth waveform is high, strongly mitigating speckle, scintillation and fade.

\subsubsection{Technical Feasibility}

There have been significant technical advances in all coherent transceiver subsystems, thereby making the proposed system tractable. First, CTI has demonstrated (patent pending) $1.5 \mathrm{~m}$ wavelength, single frequency, diffraction-limited, diode pumped high power waveguide lasers that offer from 1 to $50 \mathrm{~W}$ average power in highly efficient, compact packages. The master oscillators have also improved, with conventional linear resonator designs dropping in cost and improving in performance (100's of $\mathrm{mW}$, sub-kHz line width). CTI has also initiated detailed technical discussions with a telecommunications micro-electro-mechanical (MEMs) based master oscillator (MO) provider who has recently developed a few-gram mass, sub-watt power consumption, master oscillator that appear to have coherence adequate for the proposed 100's of meters of $\mathrm{FM}-\mathrm{CW}$ range operation. The MEMs-MO may require slight modification, but it appears to be stable and rapidly tunable for the UGV application. The receiver signal processor can be assembled from digital signal processors (DSPs) that CTI has direct experience with. Depending upon how many range reports are required, cheaper/faster/simpler DSPs can be implemented. The scanner is within the limits of designs that CTI has fielded before and is made practical by introduction of the small, few $\mathrm{mm}$ diameter aperture in the proposed transceiver.

\subsubsection{Cost-Scaling, Power-Scaling, Upgrades}

The figure shows a preliminary conceptual engineering drawing of the coherent FMCW laser radar sensor. The sensor dimensions are $42 \mathrm{~cm} \times 17 \mathrm{~cm} \times 18 \mathrm{~cm}(\mathrm{~L}, \mathrm{~W}, \mathrm{H})$. The design can be made smaller with additional effort. Smaller in many cases, means lower cost, motivating size reduction. The miniature $2 \mathrm{~mm}$ class transceiver aperture in the design reduces the scanner cost and size significantly, for example. The unit production cost estimate of the transceiver is challenging to assess without detailing environmental specifications (thermal, shock, vibration), signal processing requirements (raw vs. partially processed range data vs. end product 3D images), and interface requirements (electrical, thermal, optical, mechanical). At the risk of over-simplify- 


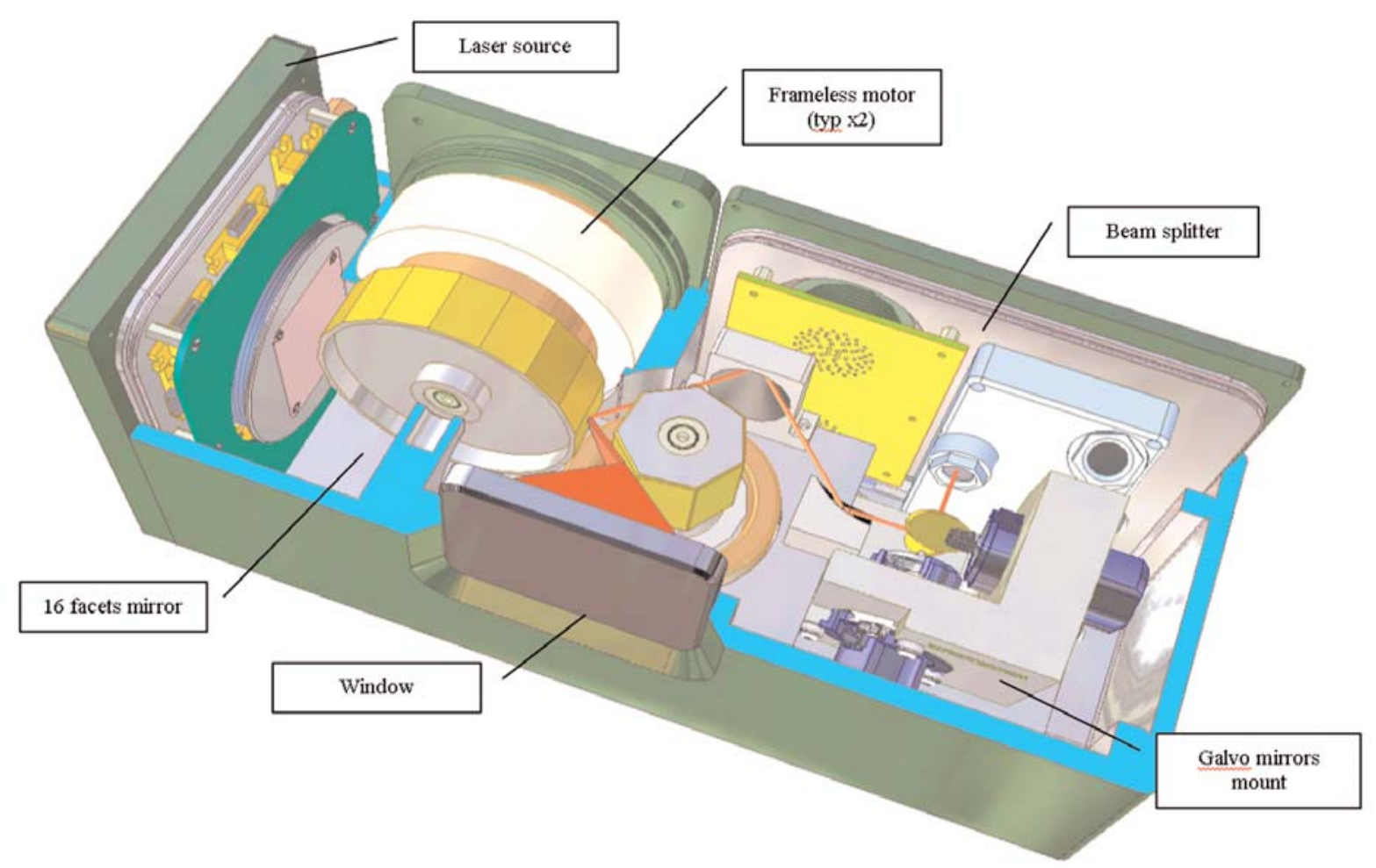

Figure 6.5: Mockup of Coherent Technologies Inc. FMCW UGV LADAR.

ing, the objective is $\$ 10 \mathrm{k}$ class transceivers in quantities of 1,000 . In yet larger quantities, more sophisticated manufacturing approaches such as custom molded optics, ASICs, roto-molded precision EMI enclosures, and other technologies offer further cost reduction.

The baseline hybrid-mount linear array receiver can be upgraded to $2 \mathrm{D}$ format (when such technology exists, and it does not yet), the transmitter can be upgraded from $1 \mathrm{~W}$ to $50 \mathrm{~W}$ average power (exists) for enhanced range performance and higher frame rates, and diversity imaging (multispectral, polarimetric, etc.) can be added straightforwardly based on designs that CTI has already developed.

Additional information on FM-CW LADAR technology is provided in Section 2.4. Further details on CTI-developed concepts for the BAA Phase I design can be requested from the following contact person: Duane Smith (duane@ctilidar.com), phone: 303379-3137.

\subsubsection{Lockheed Martin Missiles \& Fire Control}

A simple system that can meet the shorter-range requirements for autonomous navigation can be constructed using commercial parts and non-developmental items, such as Lockheed Martin's mature laser pulse signal processing electronics. Our experience led us to propose a dual-axis scanned, small linear array receiver. This approach allows us to propose a design that is small, flexible, and inexpensive. Items such as 


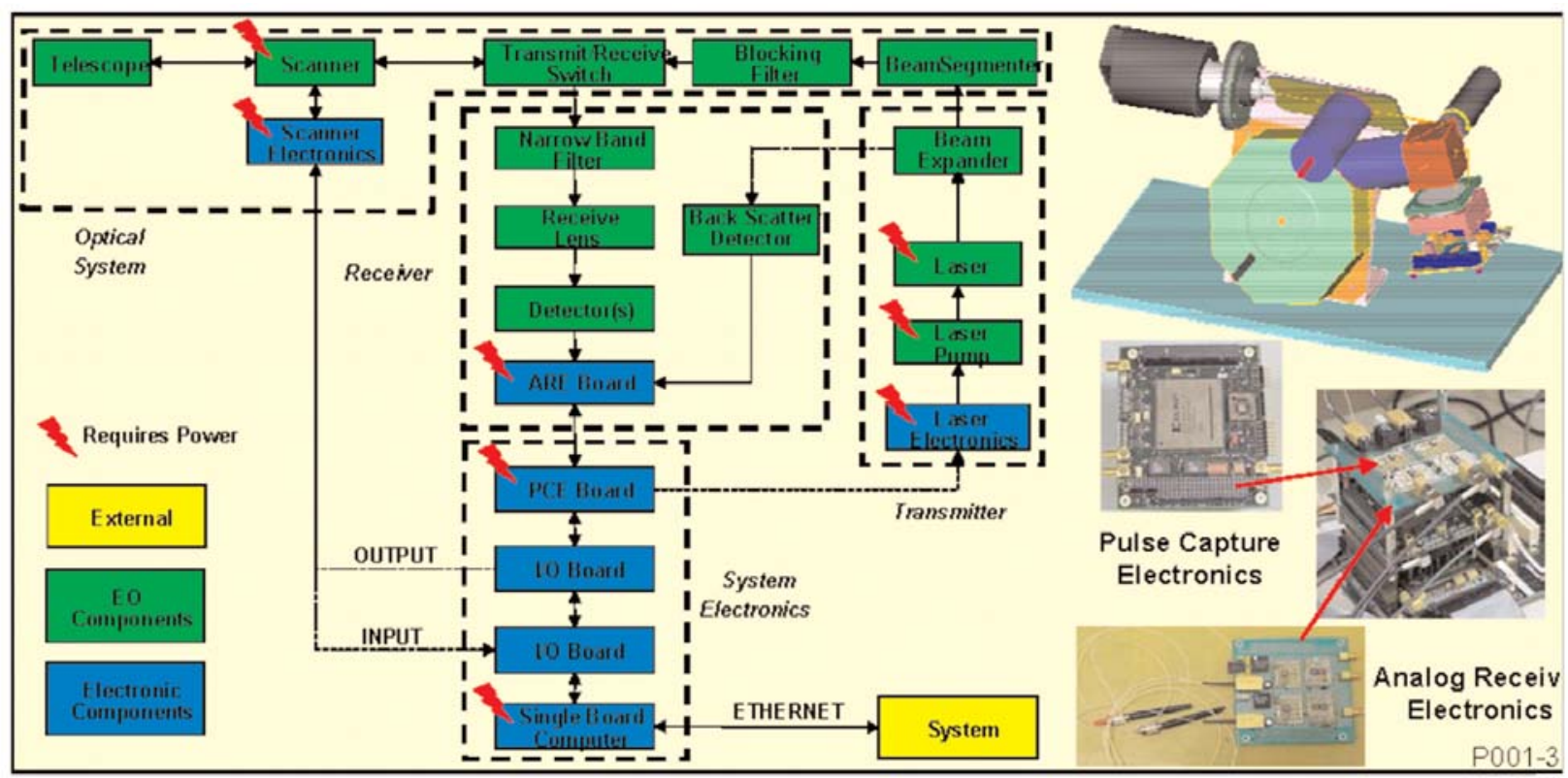

Figure 6.6: Lockheed LADAR concept uses proven system architecture, signal processing electronics, commercial components, and standard interfaces.

high-repetition rate, low-pulse-energy lasers, detectors, scanners, and processors are commercially available. An added benefit is the ability to change the scan rates to adjust resolution, FOV, and frame rates to meet mission needs without requiring complex gimbaled and/or optical designs.

The basic components of a LADAR sensor are the transmitter, receiver, optical system, and system electronics. The transmitter is a compact, high pulse rate laser (or laser diode). The optical system fully scans the laser to create an image with fine angular resolution. It also provides the collecting aperture for the InGaAs (or Silicon) detector receiver. The system electronics control the laser, scanner, process the laser returns, and provides data to update the terrain database.

A core technology of the system is Lockheed Martin's existing, flight-tested, signal processing electronics to capture and analyze laser pulses. This technology is referred to as the Pulse Capture Electronics (PCE). It is a well-established direct detection approach that accurately determines relative reflectivity (scene intensity) under varying conditions of range, atmospheric attenuation, obliquity, multiple returns, and noise. This is accomplished by matching a programmed template with the entire return pulse data to minimize the effects of signal strength variation, noise and distortions.

Additional information on the advanced LADAR technology being developed at Lockheed Martin and being offered in the BAA Phase I design can be requested from the following contact person: Bruno Evans (bruno.evans@Imco.com), phone: 972-6037945 


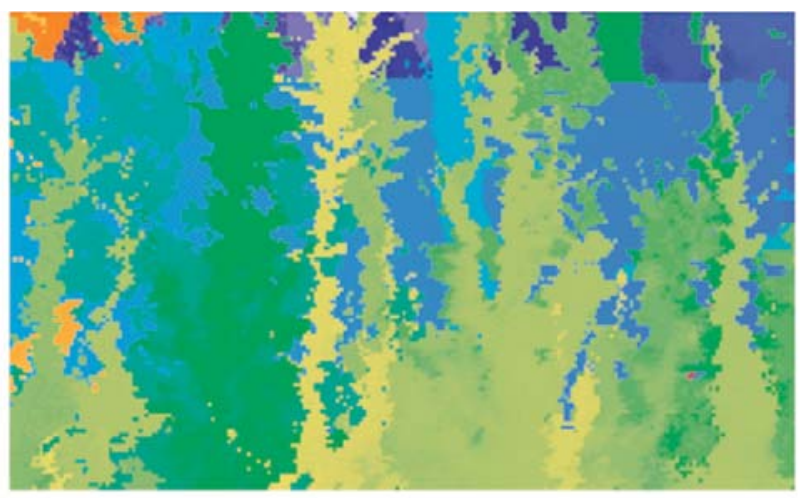

First Pulse Sees Foliage

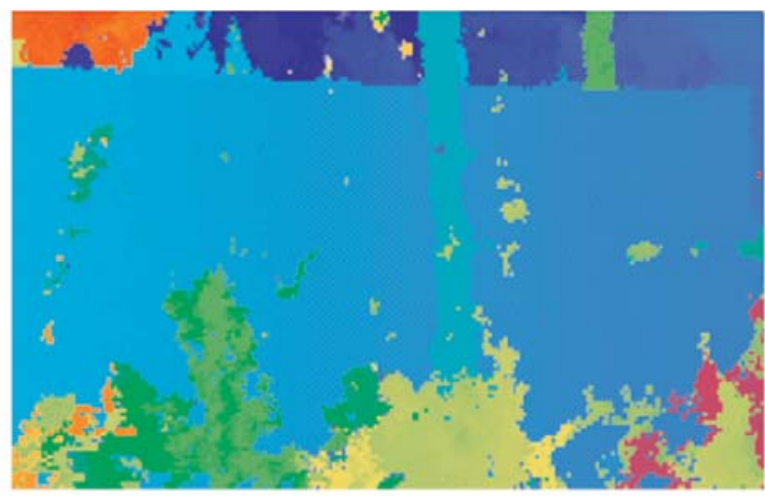

Second Pulse Sees Wall Behind Foliage

Figure 6.7: LADAR Pulse Processing. Pulse signal processing has first / best / last pulse logic for improved, single-frame, imaging performance through foliage.

\subsubsection{Raytheon Missile Systems}

Raytheon is now in the 28th month of a 42 month effort funded by the Air Force Research Laboratory, Munitions Directorate, Eglin A.F.B, FL, to generate a brassboard Flash LADAR seeker. Dr. William Humbert is the AFRL/MNGS program manager. AFRL intends to use the brassboard seeker for development of Flash LADAR autonomous target acquisition (ATA) algorithms. Delivery of the seeker to AFRL is planned for May, 2004. To generate the seeker, Raytheon is developing a 256x256 pixel $\mathrm{HgCdTe}$ flash LADAR avalanche photodiode (APD) detector array, a multi-pulse processing read-out-integrated-circuit (ROIC), and Flash LADAR seeker system components and architecture.

The Flash LADAR APD array and ROIC are well along in the development cycle. APD's and ROIC's have been designed, fabricated, hybridized and tested. All functionality has been verified. Operability's in excess of $97 \%$ have been achieved. Figure 6.8 shows elements of the detector buildup as well as the final detector configuration on a leadless chip carrier (LCC). Table 1 lists the detector performance requirements. The detector records amplitude of the first pulse, and times of arrival of the first and second pulse returns. The ROIC performs several functions including global bias, individual bias adjustment for each pixel, timing ramp generation, and signal pre-amplification. Raytheon is now under an AFRL Dual Use Science and Technology (DUST) contract to extend the ROIC to three pulse-return capability, as well as improve range accuracy and resolution by a factor of 2 . To achieve these improvements, the DUST ROIC is being designed with $0.18 \mu \mathrm{m}$ geometry, whereas the current AFRL/MNGS Flash LADAR ROIC has been designed with $0.35 \mu \mathrm{m}$. First demonstration of DUST ROIC performance will be in late 2004. 


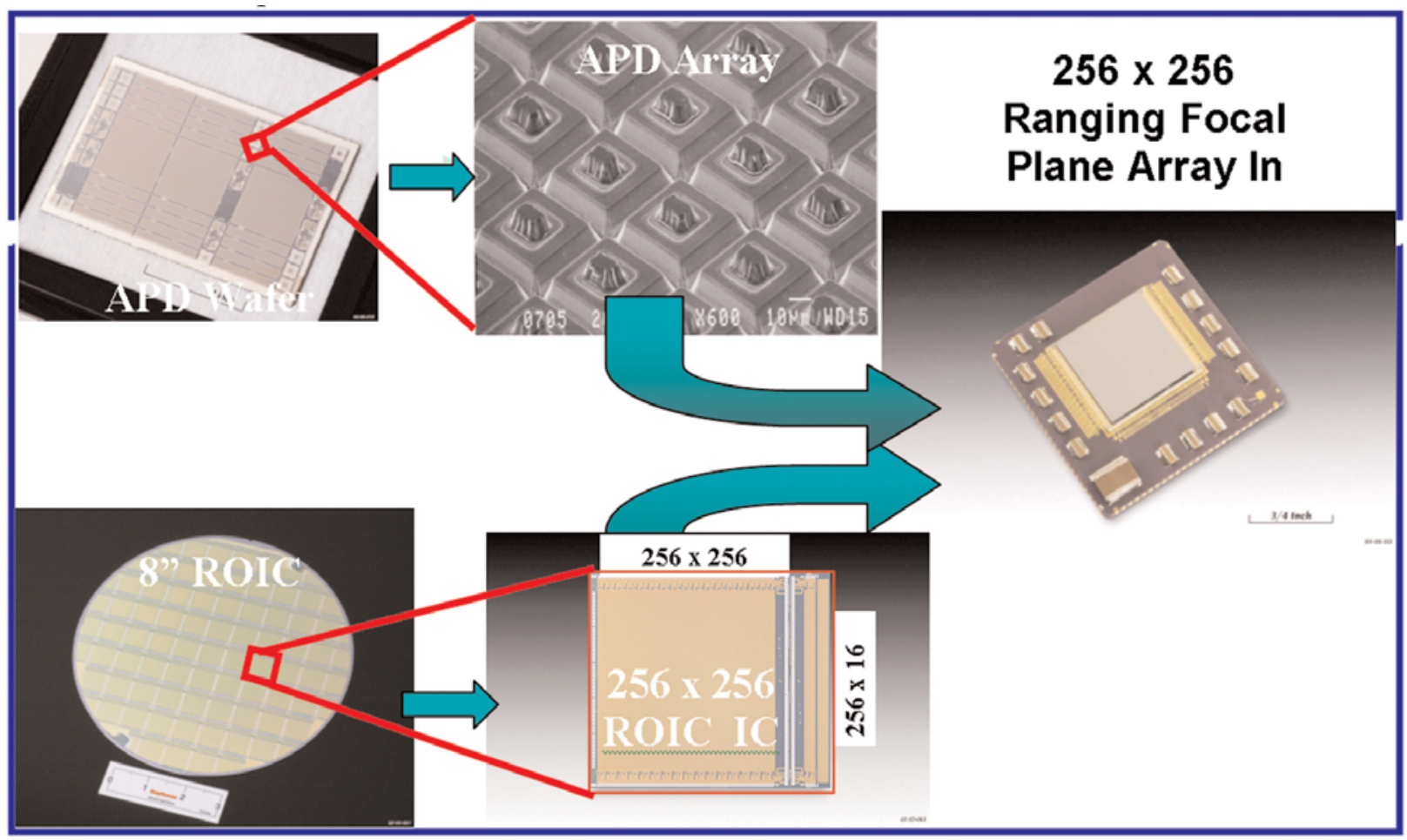

Figure 6.8: Raytheon has successfully generated the technology required to produce a fully functional, advanced, $256 \times 256$ Flash LADAR detector array.

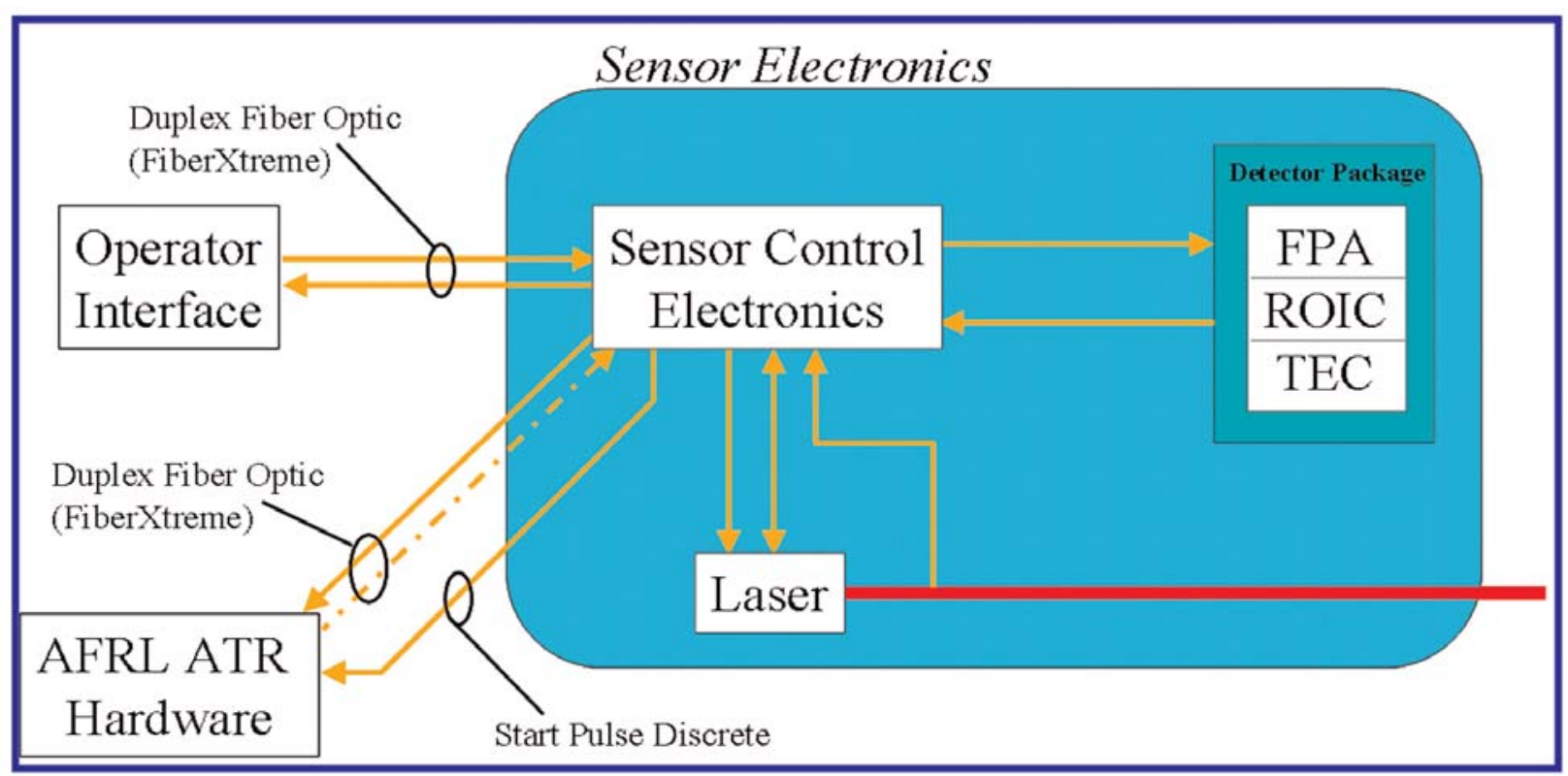

Figure 6.9: System layout for Raytheon's AFRL/MNGS Flash LADAR brassboard system. Gimbals and scanning hardware are not required to collect LADAR imagery over a large field-of- view. 
Table 6.1:AFRL / MNGS

Flash LADAR Detector Requirements

\begin{tabular}{l|c}
\hline Geometric Parameters & \\
\hline Format & $256 \times 256$ \\
\hline Pixel Pitch $(\mu \mathrm{m})$ & 60 \\
\hline Detector Optical Area $(\mu \mathrm{m} \times \mu \mathrm{m})$ & $35 \times 35$ \\
\hline
\end{tabular}

\section{EO Parameters}

\begin{tabular}{l|c}
\hline Wavelength $(\mathrm{mm})$ & 1.55 \\
\hline NEP System (pW/rt-Hz) & 0.1 \\
\hline ROIC TIA Noise Current (pA/rt-Hz) & 0.5 \\
\hline NEPD APD (pW/rt-Hz) & 0.08 \\
\hline NEP APD (nW) & 0.52 \\
\hline Bandwidth (MHz) & 100 \\
\hline Idark (pregain) (nA) & $<10$ \\
\hline K (electron to hole ionization ratio) & 0.1 \\
\hline Gain, M & $5-10$ \\
\hline Fex & 4 \\
\hline QE $\%$ & $>90$ \\
\hline Fill Factor \% & $>80$ \\
\hline Crosstalk \% & $<1$
\end{tabular}

Figure 6.9 depicts Raytheon's AFRL/MNGS Flash LADAR system concept. This brassboard sensor will be fully computer controlled, provide real-time imagery display at 10 frames per second, perform burst mode data collection/transmission at 20 frames per second, and output all LADAR data to the AFRL/MNGS ATA data processor.

Table 6.2 highlights the advantages Flash LADAR has over conventional scanned LADAR sensors for the UGV application. Raytheon believes the detector array developed under the AFRL/MNGS Flash LADAR program will serve well in demonstrating UGV LADAR technology, and that the subsequent DUST array will serve to generate a high performance, low cost, UGV LADAR production system.

Table 6.2: Advantages of Flash LADAR for UGV Applications

- Overall system simplicity and ruggedness

- Frame rates potentially reaching $1 \mu \mathrm{s}-2 \mu \mathrm{s}$

- Fine stabilization and motion compensation not required

- No motion artifacts in collected imagery

- Most electronics processing able to be carried out on single integrated circuit

Additional information on the Ladar BAA design using their Focal Plane Array technology can be requested from the following persons at Raytheon Missile Systems: Al Coit (coit@raytheon.com), phone: 520-545-9354 or Pat Trotta (patrotta@raytheon.com) 


\subsection{Strategy for Further Development}

In the near term, NIST does not expect to see the announcement of any new LADAR products that can meet the BAA LADAR requirement specified for driving unmanned ground vehicles. Therefore, the need is still there. The plan is to continue to pursue funding support for a follow-on Phase II design validation or prototype development. Based on the information gained during the Phase I design reviews, NIST believes that Phase II, if funded, should be conducted in two steps. The proposed development steps are as follows:

Step 1:

Conduct design feasibility tests on one or more of the highest scoring designs using existing hardware and software which is available from the contractors in order to qualify best approaches for further development. Duration of this step is estimated to require 9 months to 12 months to complete. Some data collection will be required during this step.

\section{Step 2:}

After selection of a qualified design, proceed with final design and fabrication of a strap-down prototype sensor. Significant sensor testing and evaluation would follow. Duration of this step in the development process is estimated to require 12 - 24 months to complete, depending on the selected design. 


\subsection{Discussion and Recommendations}

In the preceding chapters we have described the general technologies and mathematics associated with the development of practical laser radar devices. We have also attempted to summarize state-of-the-art developments in this area. We have not, by any means, covered all research and development that is underway in this field. In some cases, as in Chapter 5, we have selected examples that serve to illustrate general issues and areas where developmental work is still needed. The SPIE AeroSense conferences (now, as of 2004, known as the SPIE Defense and Security Conference, usually held in April of each year in Orlando, Florida) continue to provide a forum for this topic and readers are referred to the proceedings of those meetings for the latestbreaking technical news in this research area. Here we conclude with a collection of discussions and recommendations needed to advance next-generation LADAR for mobility, ground machine control, and construction as-built information generation.

\subsection{FOV Limitations:}

FPA "staring array" research is presently vigorous along many architectural paths. However, it is well to remember that a $256 \times 256$ pixel array remains the largest benchtested system. Referring back to the attributes of an ideal sensor for ground mobility

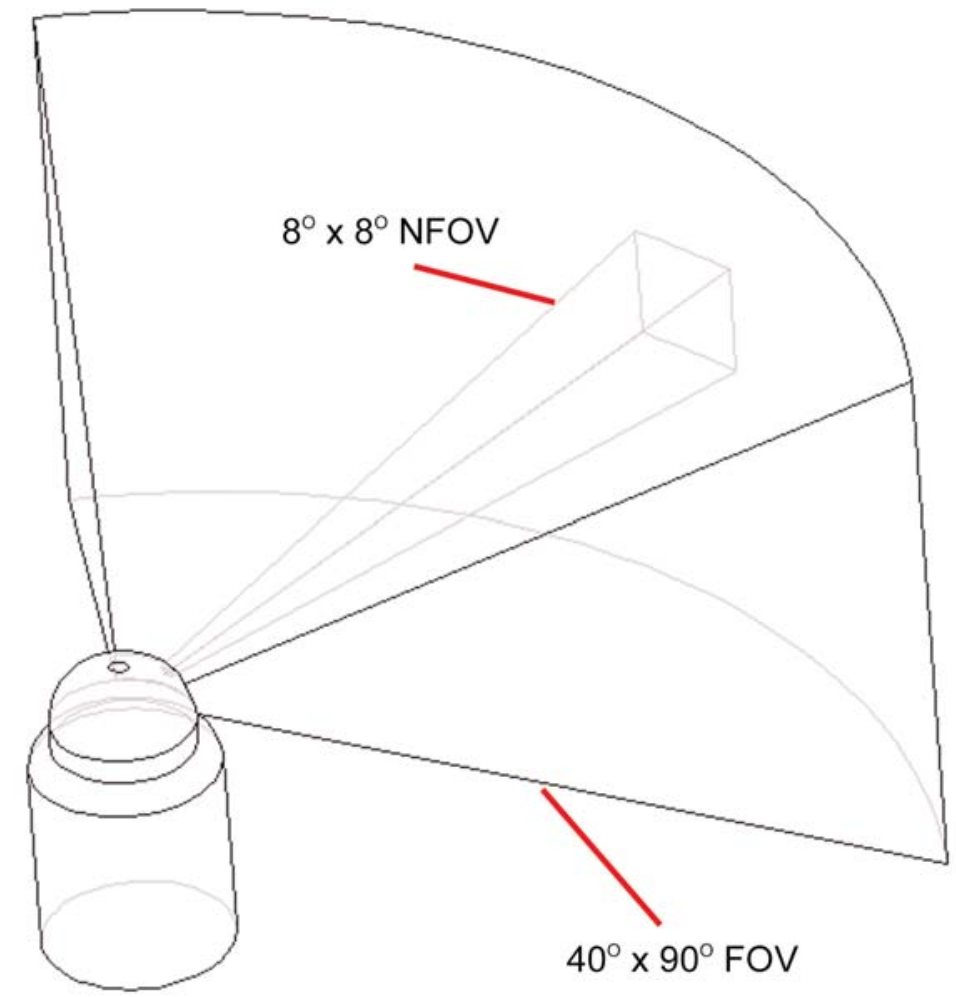

Figure 7.1: NFOV $\left(8^{\circ} \times 8^{\circ}\right)$ for a $256 \times 256$ pixel FPA with an angular resolution of 0.03 degrees compared with a typical desired mobility navigation window $\left(40^{\circ} \times 90^{\circ}\right)$. and machine automation presented in Table 1, we would find that for an angular resolution of $0.03^{\circ}$ this would translate into an FOV of around 80 $x 8^{\circ}$. A typical minimum steering and navigation FOV for mobility would be $40^{\circ} \times 90^{\circ}$. The difference between these two is shown graphically in Figure 7.1. As a consequence, except for very limited military applications (e.g. UAV foveal targeting) unrelated to ground vehicle mobility and ground machine control, the FPA sensors on the near-term horizon will require scanning mechanisms for any practical implementation. This 
requirement is more severe in the case of precision machine control and construction operations where a $0.03^{\circ}$ angular resolution is currently considered "coarse" -- existing scanning LADARs (e.g. see page A.14 in Appendix A) provide angular resolutions of $0.002^{\circ}$, more than an order of magnitude more refined. Such angular precision (with commensurate range accuracy in the $1 \mathrm{~mm}$ to $3 \mathrm{~mm}$ regime) is required where realtime object recognition and automated machine acquisition and transfer of construction components is the end objective. At that angular resolution $\left(0.002^{\circ}\right)$ a $256 \times 256$ staring array would cover only a $0.5^{\circ} \times 0.5^{\circ}(9 \mathrm{mrad} \times 9 \mathrm{mrad})$ FOV. Mechanical scanning mechanisms, including galvos, rotating polygons, and Risley prisms (see Section 3.4) have all been proposed or implemented in various forms for steering of FPA staring arrays. All represent complexities that ultimately control the size and mass of the sensor since the optics and beam deflection subsystems dominate the overall system design. These are discrete components that are difficult to shrink in their present manifestations and manufacturing approaches and as such will remain bulky and costly. They are ultimately dead ends for Next-Generation LADAR, which seeks to achieve coffee-cup size sensors at sub- $\$ 1 \mathrm{~K}$ production pricing.

\subsection{Timing Issues:}

For pulse time-of-flight ranging devices -- which is the technology being utilized in most FPA systems now being designed with a range in excess of a few meters -- detector

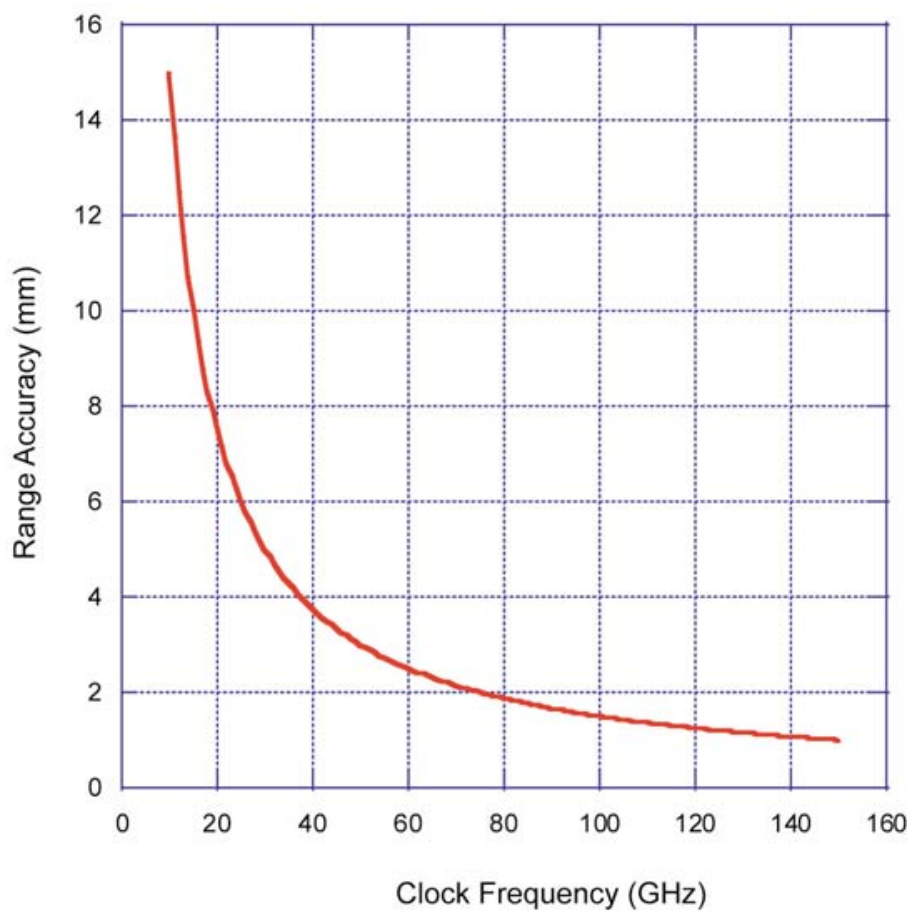

Figure 7.2: Range accuracy for pulse time-of-flight LADAR as a function of timing accuracy (clock bandwidth). This represents round-trip bin accuracy with no consideration for sub-bin "super resolution." and digitizer bandwidth is everything. At present, one can purchase off shelf telecom digitizers and APDs that have $2 \mathrm{GHz}$ bandwidth. Beyond that there are a number of high-end scientific digitizers, developed largely for the particle physics community, that approach $10 \mathrm{GHz}$ sample rates. But what we want, in reality, is a picosecond clock and photon detector in the $1.55 \mu \mathrm{m}$ regime. There are photodetector chemistries -- mainly GeSi -- that are potentially capable of being 
pushed into the near $100 \mathrm{GHz}$ regime. That leaves the question of developing a picosecond on-chip timer. A 1 ps timer is presently not achievable in silicon. A silicon CMOS circuit (of the type currently being used in the ROIC of most LADAR systems) is effectively a group of devices that, when turned on, look like resistors that either charge up or discharge adjacent capacitors that provide input into the next stage of a logic circuit. As technology advances (Moore's Law) the minimum discrete component size shrinks. As they shrink their RC time constants shrink, producing faster switching speeds. If one investigates $\mathrm{GaAs}$, which has a less mature fabrication technology, one finds that the circuits that can be built will have a smaller number of transistors per unit area. GaAs does not have the native oxide that silicon has and therefore it is not possible to grow ultra-thin oxide layers to produce low power capacitive elements that are crucial to precision timing devices.

In silicon, as elsewhere, we are limited by feature size. The best commercial CMOS process, currently offered by Taiwan Semiconductor, is $0.35 \mu \mathrm{m}$. In this regime a CMOS inverter will introduce a delay in the $100 \mathrm{ps}$ range. Such an element could become part of a string of such devices forming a delay line for an individual pixel in an FPA. One could further envision having a counter that keeps track of the clock cycles following receipt of a trigger pulse. The trigger pulse edge can be propagated down a string (a long one) of tristate inverters. The arrival of the return pulse (reflected from a distant target) edge can then be used to freeze the inverter outputs.

One can then determine how far down that delay line the trigger pulse edge propagated when photon detection signal arrived. Using this approach it would be feasible to digitize time of flight down to the $100 \mathrm{ps}$ level. Since that is round trip flight time, the range bin size is $15 \mathrm{~mm}$. Design of such a system would be dominated by consideration of noise and jitter sources associated with the large number of inverters required.

The objective, in this brute force approach, is to cram more circuitry in to the pixel unit cell. But that has a price in that each pixel becomes larger which ripples down to the requirement for larger optics and so forth. VISA and Z-stacking may alleviate some of that real estate demand but will not eliminate it. New silicon processes are on the horizon: $0.25 \mu \mathrm{m}, 0.18 \mu \mathrm{m}$ and even $0.13 \mu \mathrm{m}$ technology is considered likely within the next few years. And the smaller the achievable component size, the faster the timer. But speed is not linear with technology size. Inverter delay is also a function of power supply voltage. With a larger drive voltage the transistors are turned on harder and thus switch faster. There is a limit where gate oxides break down. Impact ionization and high electric field strengths in the transistor channel will adversely counter and eventually limit the achievable improvements. Nonetheless, some have suggested that through aggressive silicon-on-insulator processes gate delays might be reduced to the sub-30 ps range. It may be proven over the next few years that VISA designs, with $1 \mu \mathrm{m}$ inter-wafer vias, might be able to capitalize on such aggressive processes. Zstacking solutions may be limited because all inter-layer connections must take place on the wafer periphery. When one achieves a timing accuracy below 30 ps the accuracy will begin to be limited by such things as the "jitter" of the photon detector. 


\subsection{Illumination Source Limits:}

Edge detection of the trigger and return illumination pulse is an inherent component of LADAR system accuracy. Simplistically stated, the shorter the illumination pulse, the greater the ability to resolve the time of the receipt of the pulse. Figure 7.3 illustrates this point. Microchip laser illuminators, particularly diode-pumped NdYAG designs using $\mathrm{Cr}^{4+}$ :YAG saturable absorber layers, have been developed that achieve 218 ps minimum pulse width (e.g. Albota et al, 2001). This presently represents the shortest pulse laser illuminator implemented in a practical next-generation LADAR-compatible size. Laboratory benchtop-scale devices with fs-level output pulse widths are available commercially -- and more exact systems have been developed in various laboratories -

-- but these are large,

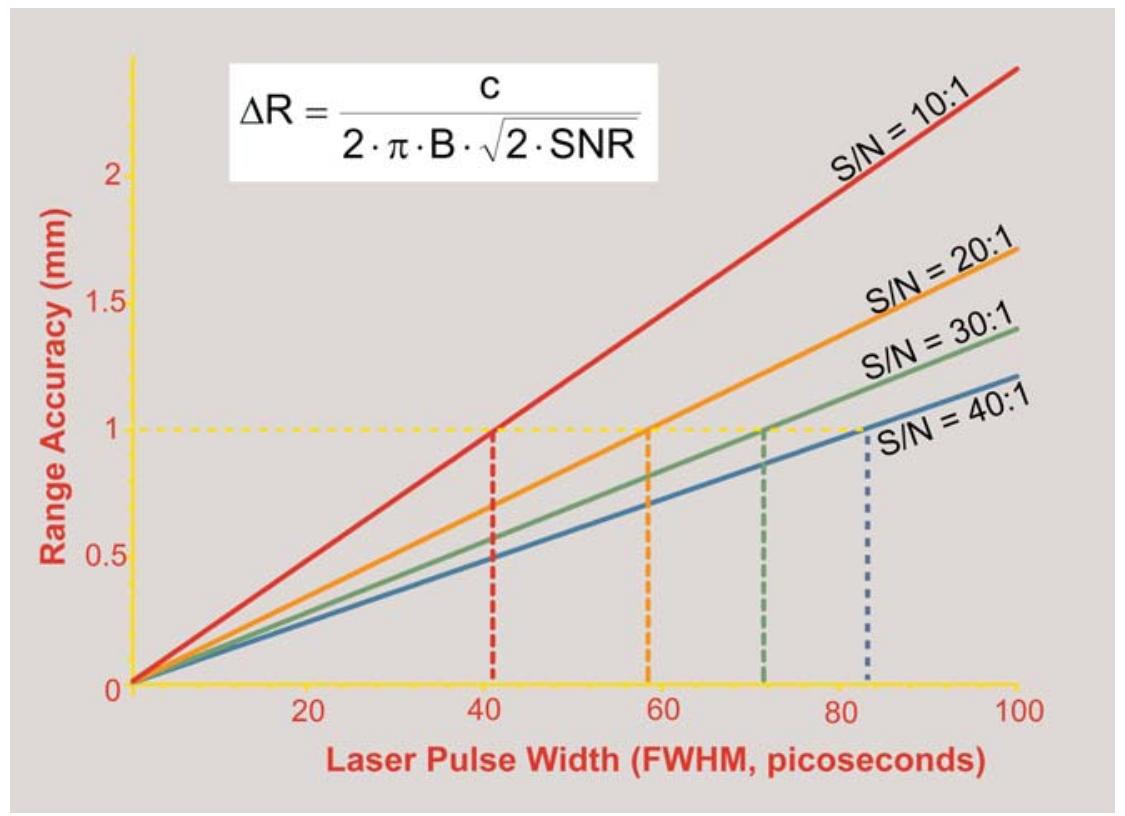
expensive, and generally produce a continuous string of such pulses through cavity resonance and interference. Due to the nature of the presently employed pulse generation methods the unambiguous range (for a LADAR ranging system) between pulses in a fslevel laser source is currently limited to under 2 $\mathrm{m}$.

A great deal of effort is expended in the development of "sub-bin" and "super-resolution" techniques that in effect attempt to model the

Figure 7.3: Range accuracy as a function of the pulse width of the laser illuminator used in a pulse time-of-flight LADAR.

information to assist in return pulse. Regardless of the efforts placed in the development of ps-level timing circuits (see 7.2 above) these are inherently limited by the accuracy with which the stopwatch "start" and "stop" events are able to be detected. Clearly, a more elegant solution to super-resolution approaches would be the development of micro-chip level fs pulsed laser illuminators, or fs-level continuous pulse illumination generators with cavity resonators that produce unambiguous ranges in the neighborhood of $100 \mathrm{~m}$ to 200 $\mathrm{m}$. 


\subsection{Performance Metrics and 3D Scene Generation Issues:}

Thus far all discussion in this report has dealt with LADAR sensor technology. There is a parallel series of issues that are most germane to the construction automation sector but eventually will have direct consequences for any LADAR destined for a market for which accountability is required. To illustrate the nature of the issue, Figure $7.4 \mathrm{a}$ ) shows a typical high-resolution scan of a HMMWV vehicle with false-color elevation scaling. A flat panel on the vehicle was identified for study (as indicated by the yellow rectangle). The returns from this area were extracted and then rotated 90 degrees so that the relative viewpoint would be looking down on the edge of the panel vertically. In an ideal data set the panel would appear as a straight line from this viewpoint. There is, however, a clear distribution associated with the actual LADAR response. This distribution is approximatey gaussian. The reported accuracy -- from sales literature -- of the instrument employed was $+/-20 \mathrm{~mm}$ with no further reference. From the data, however, it is evident that this would represent approximately $1 \sigma$ - one standard deviation. For certain applications this would be considered extraordinarily accurate; for others it would be of significant concern. Yet there is no way for the user of such an instrument to presently know in advance all of the performance metrics for any given LADAR. In many establishments that use these instruments for manufacturing metrology extensive calibration facilities must be established just to ascertain what the data being supplied by the LADAR mean. Calibration procedures are considered highly proprietary to instrument manufacturers (Cheok et al., 2003), largely because illumination source, beam deflection, and sensor idiosyncracies are frequently non-linear. Some instruments come with embedded lookup error compensation models with more than 50 correction coefficients. In the more accurate devices these models will include in addition to spatial data, in-situ temperature, pressure, and humidity.

The data shown in Figure 7.4 represent a near ideal situation for LADAR: a flat, highly reflecting panel at medium range and perpendicular to the range vector. The variance for this device (and any other LADAR) will degrade with reduced reflectivity, range, angle of incidence, surface texture, and in many cases the ambient light level to name just a few parameters that quantify the performance metrics for any given instrument. It is presently the "wild, wild west" in the commercial sector and there exists no neutral facility by which claims of performance can be verified. NIST has undertaken efforts in this area (Cheok, 2003) to establish a national LADAR performance metrics facility and a first industry-government workshop was held at NIST in June of 2003 to discuss the nature of such a facility. The general concensus is that three facilities are required: a refined environmentally controlled test range for sensors that operate at very high levels of accuracy $(<100 \mu \mathrm{m})$ within short ranges; a mid-range environmentally controlled facility for instruments up to $50 \mathrm{~m}$ range; and an outdoor test course for autonomous machinery sensors and mobility platforms that has the capability to generate near synchronous ground truth for any given weather and seasonal consideration. Such a facility would also include calibrated dynamic objects that move about the scene during a mobility platform test. NIST is proceeding to develop the second facility in FY04/ FY05. 


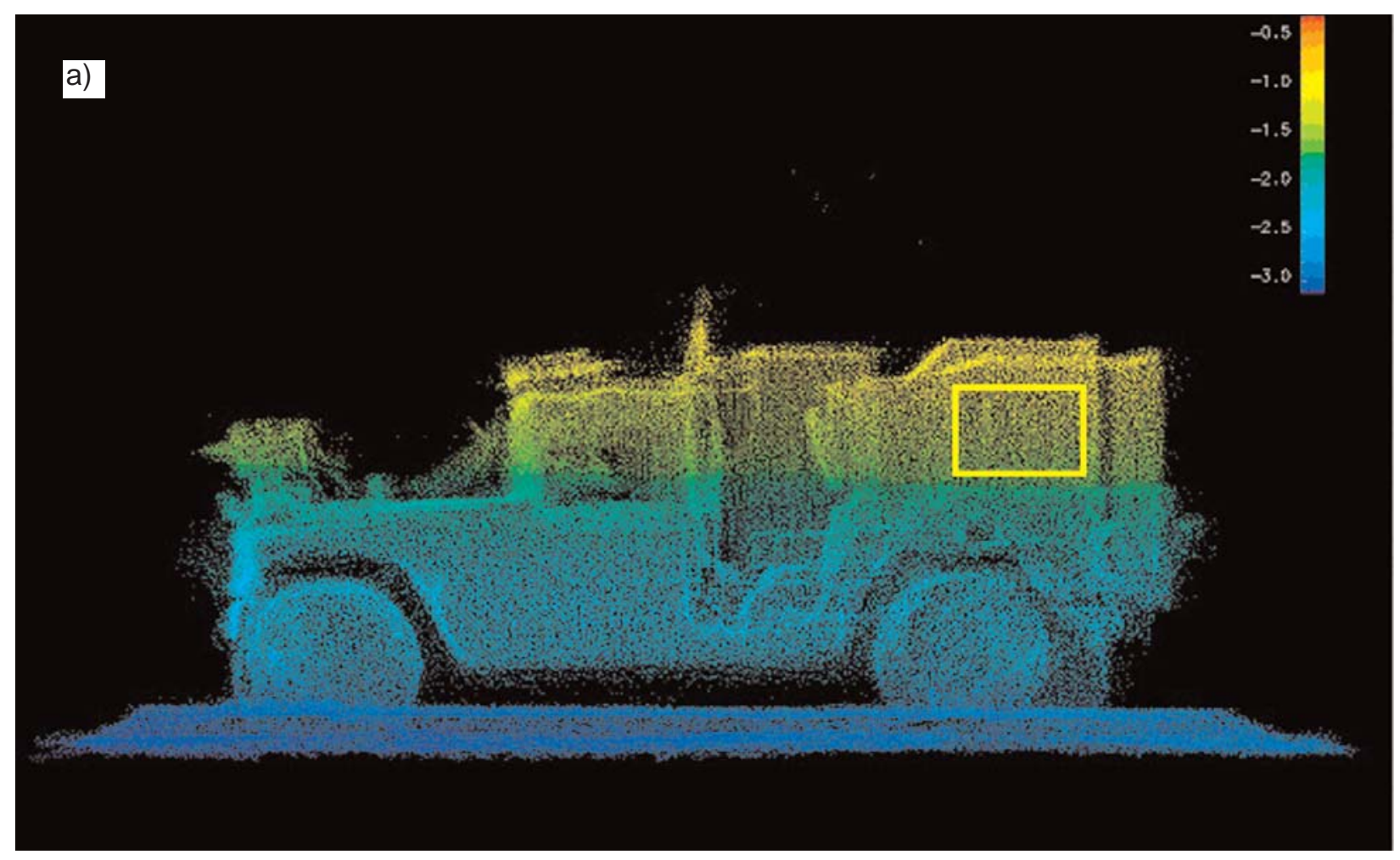

Figure 7.4: Not all LADAR performance is the same. At present, it is the "wild, wild west" with respect to LADAR performance metrics. There are no national (nor international) standard tests that have been established by which LADAR systems can be uniformly benchmarked. When an accuracy is quoted for a LADAR system, what is its basis? The HMMWV scan shown above was acquired with a sensor for which the stated accuracy was $20 \mathrm{~mm}$. Subsample data were extracted from the flat utility panel designated by the yellow rectangle in a) above. In b) below, those data were rotated 90 degrees on the computer screen so that the view is looking down on the data sample. A flat plate (which is what was scanned) would have produced a straight line for perfect data. Instead there is a near Gaussian distribution for which the stated accuracy probably represents one standard deviation.

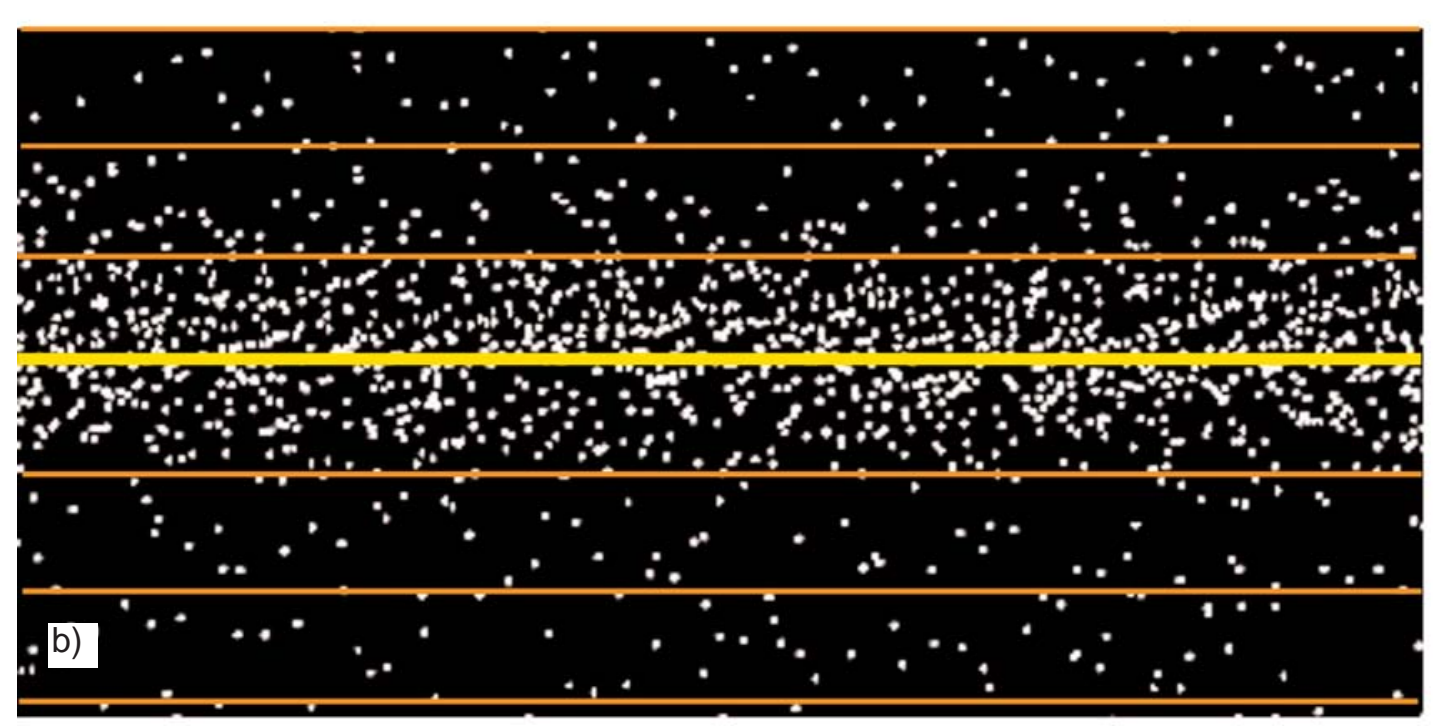

$60 \mathrm{~mm}$

$40 \mathrm{~mm}$

$20 \mathrm{~mm}$

Truth

$-20 \mathrm{~mm}$

$-40 \mathrm{~mm}$

$-60 \mathrm{~mm}$ 

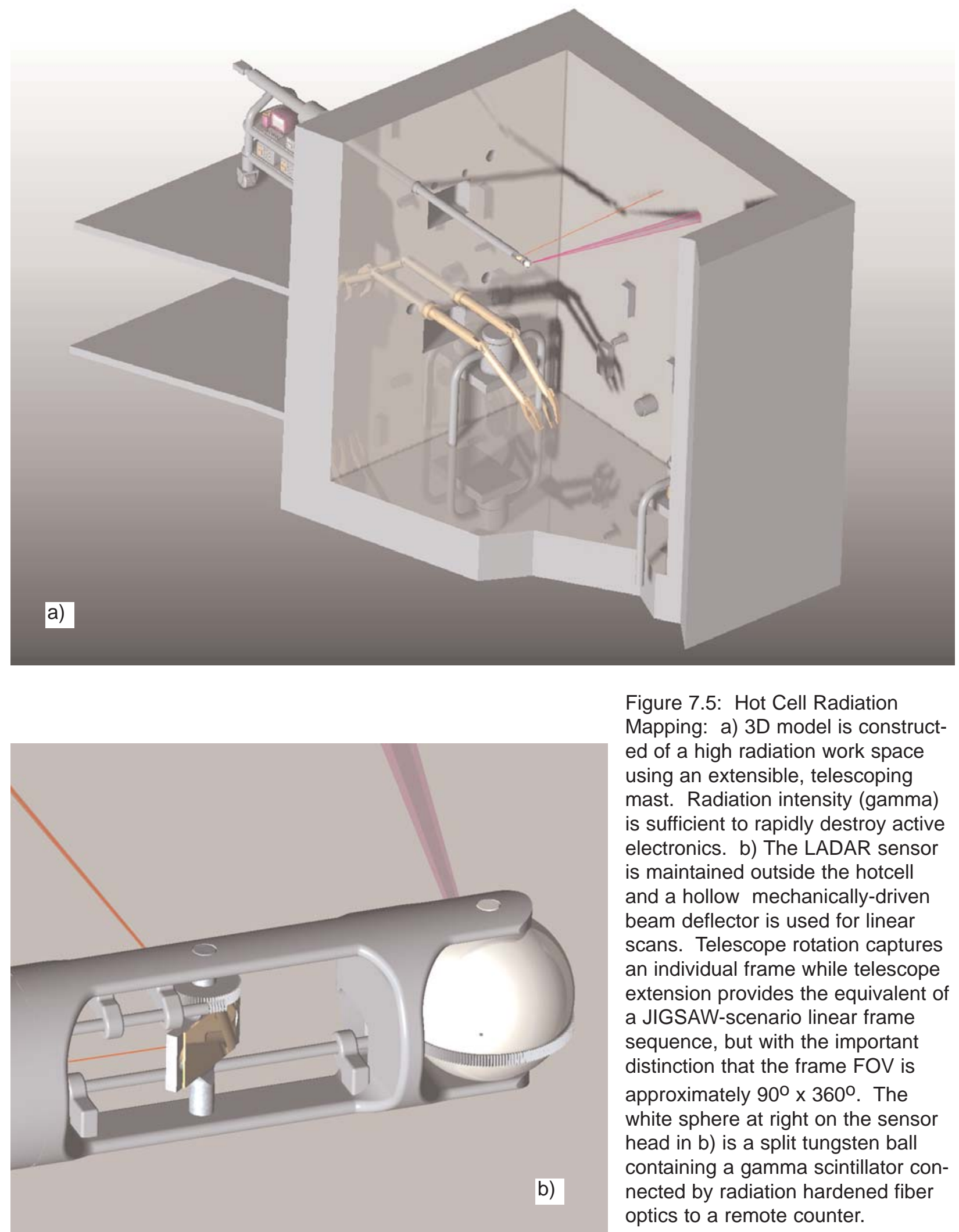

Figure 7.5: Hot Cell Radiation Mapping: a) 3D model is constructed of a high radiation work space using an extensible, telescoping mast. Radiation intensity (gamma) is sufficient to rapidly destroy active electronics. b) The LADAR sensor is maintained outside the hotcell and a hollow mechanically-driven beam deflector is used for linear scans. Telescope rotation captures an individual frame while telescope extension provides the equivalent of a JIGSAW-scenario linear frame sequence, but with the important distinction that the frame FOV is approximately $90^{\circ} \times 360^{\circ}$. The white sphere at right on the sensor head in b) is a split tungsten ball containing a gamma scintillator connected by radiation hardened fiber optics to a remote counter. 


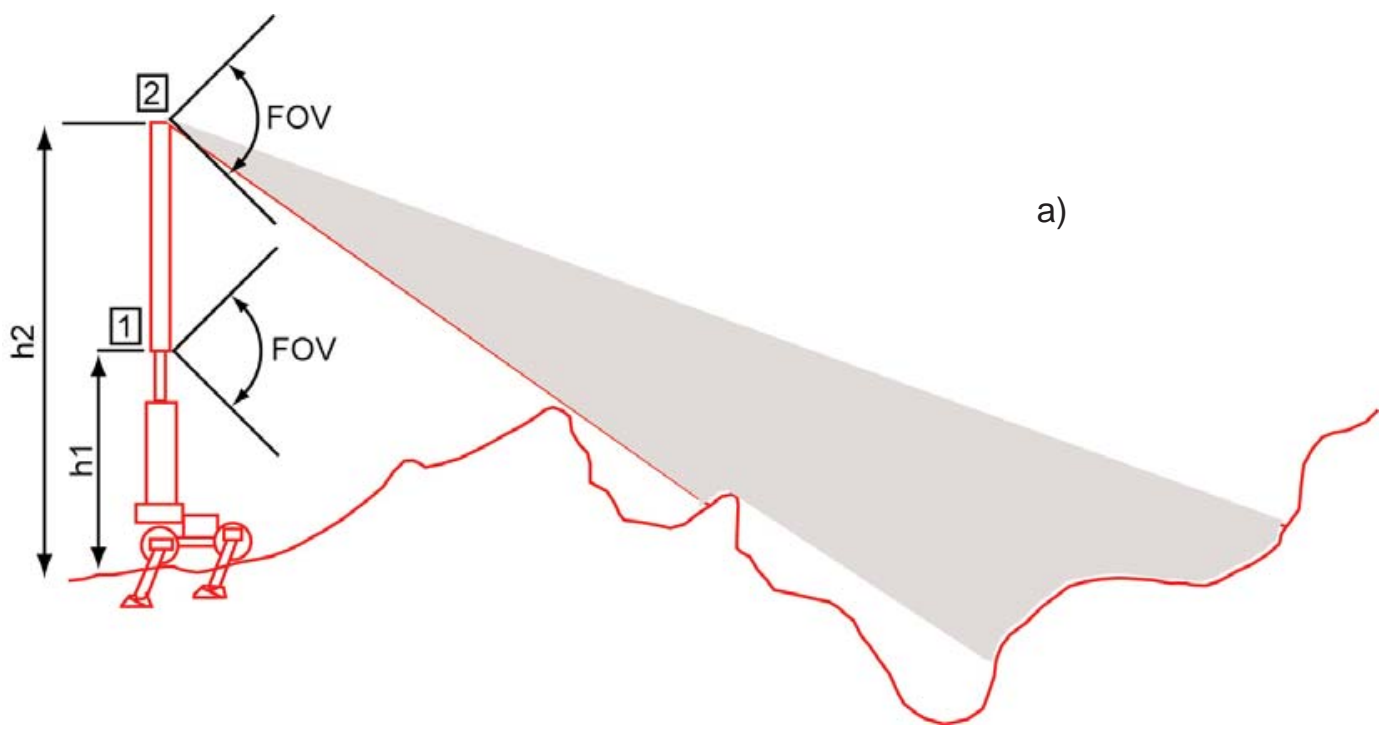

Figure 7.6: Automated As-Built Construction Site Model Generation. a) An autonomous site scanning system equipped with telescoping sensor pod provides more complete models to be developed than with traditional tripod-mounted surveying techniques. The grey area represents scene data unknown to the instrument at scan location 1, but visible to location 2; b) Complicated sites require registration of scores or hundreds of scans to produce legally binding survey records. These invoke a need for performance metrics evaluation from a neutral source.

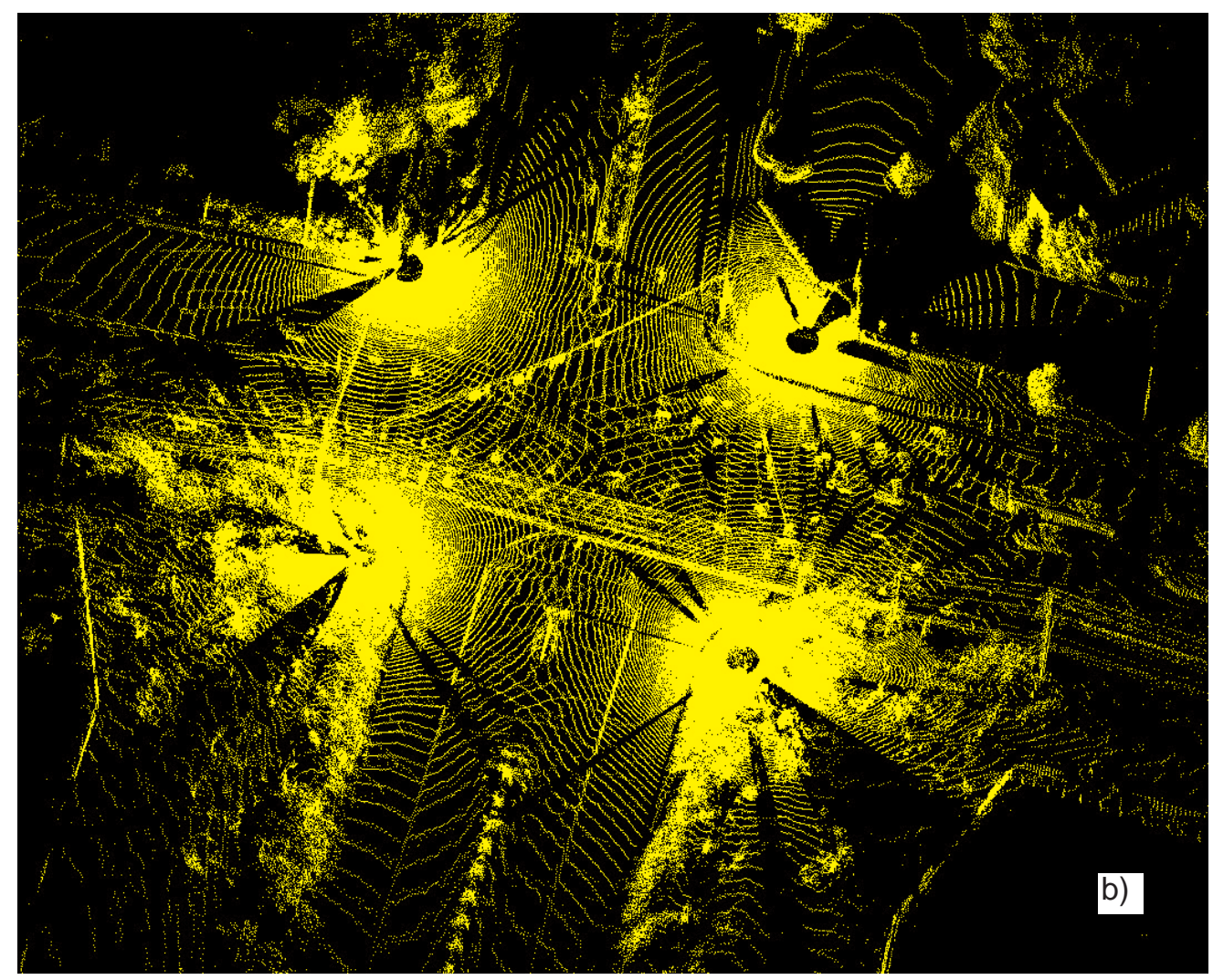


As the research community moves towards Next-Generation LADAR it is well to consider that the designs should be versatile enough to permit migration to non-standard uses. The reason for doing this is largely a matter of economics: LADARs presently range in price between $\$ 25,000$ to $\$ 400,000$. At these rates the market penetration will remain small and small production lot manufacturing approaches will control pricing. Figures 7.5 and 7.6 (extracted from Stone, 2003) provide two unusual examples of many possible alternative scenarios where low-cost, next-generation LADAR would be enabling.

Figure 7.5 describes the concept of tracking the location of high radiation zones within a traditional nuclear hot cell. In Figure 7.5a a 3-D model is constructed of a high radiation work space using an extensible, telescoping mast. Radiation intensity (gamma) is sufficient to rapidly destroy active electronics, so the LADAR illuminator and detector cannot be inside the cell. Instead, as in Figure 7.5b the LADAR sensor is maintained outside the hotcell and a hollow tube allows a mechanically-driven beam deflector to allow acquisition of linear scans. Rotation of the telescoping tube about its axis allows for the capture of an individual frame while telescope extension provides the equivalent of a Jigsaw-scenario linear frame sequence, but with the important distinction that the frame FOV is approximately $90 \times 360$ degrees. The white sphere at right on the sensor head in Figure $7.5 \mathrm{~b}$ is a split tungsten ball containing a gamma scintillator connected by rad-hard fiber optics to a remote counter. The entire device comprises the equivalent of a 3-D borescope but with the important distinction that 3-D geometry is acquired as well as scalar data (in this case, gamma radiation intensity) that can be subsequently mapped to the geometry for the purpose of automated decision making and programming of machinery (e.g. robot arms) that operate within that environment.

Automated as-built construction site model generation is discussed in Figure 7.6. Unlike aerial (UAV, OAV) frame sequence capture -- which is inherently a linear collection of "2-1/2 D" frames -- construction site component representation requires a full 3$\mathrm{D}$ model for both refined object detection in preparation for automated machine handling, as well as to establish legally binding survey information on the geometric state of the infrastructure. Future automated survey vehicles will self-register to the site coordinate system and will use extensible masts to improve view factor before moving to the next pre-designated scan point. The grey section in Figure 7.6a represents terrain not viewable at state 1 . Registration of hundreds of such frames collected from key locations around the construction site are then registered to produce a site model such as the traffic intersection shown in Figure 7.6b. Already, work is underway at NIST to equip construction cranes with real-time LADAR for the purpose of steel component placement (Lytle et al, 2003). In this scenario, the crane makes use of two independent $10 \mathrm{~m}$ range FPA LADARS (CSEM SR-2 -- see Appendix A) for the purpose of target recognition, geometric lock-on, and real-time trajectory control. The intent is, in addition to the immediate instance of local machine vision enhancement, to lead towards the concept of each piece of machinery operating on a construction site to become an independent gatherer of 3-D state information. Such information will be maintained in a general 4-D repository for use by other machines and humans. 


\subsection{Recommendations for further Research:}

In summary, we recommend that vigorous research be conducted in the following areas related to Next-Generation LADAR, in order of importance:

- On-chip technology that can achieve 10 ps timing resolution

- Miniaturized and distributed beam-folding and beam deflection array systems

- Eye-safe photodiodes that can operate at $150 \mathrm{GHz}$ bandwidth

- Compact fs pulse illuminators

- Compact, ultra-linear, ultra-wideband FM-chirp generators for CLR devices

- High power VCSEL (surface multi-laser emitters,) wafers and optical couplers

- Standardized means for assessment of LADAR performance metrics

One can modify and tailor the above topics to suit the desired end target - whether it be mobility LADAR for unoccupied ground vehicles; the control of construction machinery; or the control of manufacturing processes. In all these variations the common requirement for small, inexpensive, accurate, and fast LADAR systems will force one's attention on the above areas of research. 


\subsection{References}

\subsection{General References}

Albota, M.A., Aull, B.F., Fouche, D.G. et al., "Three-Dimentional Imaging Laser Radar Using Geiger-mode Avalanche Photodiode Arrays and Short-pulse Microchip Lasers," Lincoln Laboratory Journal, Volume 13, Number 1 (2001).

Aull, B.F. et al., "Geiger-Mode Avalanche Photodiodes for Three-Dimensional Imaging," Lincoln Laboratory Journal, Volume 13, Number 1 (2001).

BesI, P.J., "Active, Optical Range Imaging Sensors," Machine Vision and Applications 1:127-152 (1988).

Buxbaum, B., and Gollewski, T., "Feasibility Study for the Next-Generation Laser Radar Range Imaging Based on CMOS PMD Technology," Special Contract Report to the National Institute of Standards and Technology, NIST, Gaithersburg, MD 20899, pp. 65. October 30, 2002.

Cheok, G. et al., "Proceedings of the LADAR Calibration Workshop June 12-13, 2003," NISTIR-7054, October 2003, National Institute of Standards and Technology, Gaithersburg, MD, 20899.

Frohlich, C., Mettenleiter, M., Hartl, F., Dalton, G., and Hines, D., "Imaging Laser Radar for 3-D Modeling of Real World Environments," Sensor Review, Volume 20, No. 4, pp 273-281 (2000).

Kammerman, G.W., "Laser Radar," Vol. 6 (Active Electro-Optical Systems), Chapter 1, The Infrared and Electro-Optical Systems Handbook, Clifton S. Fox, Editor, SPIE Optical Engineering Press, Bellingham, Washington, USA (1996).

Lange, R., "3D Time-of-Flight Distance Measurement with Custom Solid-State Image Sensors in CMOS/CCD-Technology," Doctoral Dissertation, Dept. of Electrical Engineering and Computer Science at University of Siegen, Siegen, Germany, June 28, 2000.

Lytle, A.M., Saidi, K.S., Stone, W.C., and Scott, N.A., "Towards an Intelligent Job Site: Status of the NIST Automated Steel Construction Test Bed," Proceedings of ISARC 2003 (International Symposium on Automation and Robotics in Construction), Eindhoven, Netherlands. September 22-24, 2003.

Schilling, B.W., Barr, D.N., Templeton, G.C., Mizerka, L.J., and Trussell, C.W., Multiple-return laser radar for three-dimensional imaging through obscurations, APPLIED OPTICS, Vol. 41, No. 15 pp 2791-2799, May 2002 
Spirig, T., "Smart CCD/CMOS Based Image Sensors with Programmable, Real-Time, Temporal and Spatial Convolution Capabilities for Applications in Machine Vision and Optical Metrology," Doctoral Dissertation, Swiss Federal Institute of Technology, Zurich, (1997).

Stann, B.L., Ruff, W.C., and Sztankay, Z.G., "Intensity-Modulated Diode Laser Radar using Frequency-Modulation / Continuous-Wave Ranging Techniques," Optical Engineering, November 1996.

Stettner, R., and Bailey, H.W., "3D Imaging Laser Radar," United States Patent 6,133,989, October 17, 2000.

Stettner, R., and Bailey, H.W., 3D Imaging Laser Radar, U.S. Patent 6,133,989, Advanced Scientific Concepts, Inc. October 17, 2000.

Langer, D., Mettenleiter, M., Härtl, F., and Fröhlich, C., Imaging Ladar for 3-D Surveying and CAD Modeling of Real World Environments, The International Journal of Robotics Research, Vol.19, No.11, Sage Publications, Inc., pp.1075-1088, November 2000.

\subsection{Micromirror References}

Sweeney, M., Rynkowski, G., Ketabchi, M., and Crowley, R., "Design Considerations for Fast Steering Mirrors (FSM's)," Axys Technologies Imagins Systems, 2909 Waterview Drive, Rochester Hills, Ml 48309-4600.

Dagalakis, N. G., LeBrun, T., Lippiatt, J., "Micro-Mirror Array Control of Optical Tweezer Trapping Beams," 2nd IEEE Conference on Nanotechnology, Washington DC, August 2002.

\subsection{Coherent Ladar References:}

Burton, R.R., Schott, J.R., and Brown, S.D., "Elastic LADAR Modeling for Synthetic Imaging Applications," SPIE (2002).

Gatt, P., Thompson, J.A., and Henderson, S.W., "Coherent Laser Radar Range Precision for Range Resolved and Unresolved Targets, Corporation White Paper, Coherent Technologies Inc., 655 Aspen Ridge Drive, Lafayette, CO 80026 (2003).

Gatt, P., "Waveform Range and Velocity Resolution and Precision and Time-

Bandwidth Product, Corporation White Paper, Coherent Technologies Inc., 655 Aspen Ridge Drive, Lafayette, CO 80026 (2003).

Shapiro, J.W., "Performance Analysis of Peak-Detecting Laser Radars, "SPIE Vol. 663, pp. 38-56 (1986). 
Van Trees, H.L., "Detection, Estimation, and Modulation Theory, Part 1, Wiley and Sons (1968).

\subsection{Scanning References}

Montagu, J., and DeWeerd, H., Optomechanical Scanning Applications, Techniques, and Devices, Vol. 3 (Electro-Optical Components), Chapter 3, The Infrared and Electro-Optical Systems Handbook, William D. Rogatto, Editor, SPIE Optical Engineering Press, Bellingham, Washington, USA (1996).

Amatucci, E., Dagalakis, N.G., Kramar, J.A., Scire, F.E., "Performance Evaluation of a Parallel Cantilever Biaxial Micropositioning Stage," American Society of Precision Engineering, 15th Annual Meeting 2000, Scottsdale, Arizona, October 2000.

Applied MEMS, "DuraScan Mirror Technology,"

http://www.appliedmems.cc/htmlmems/index.htm (2004).

Beheim, G., Fritsch, K., "Range finding using frequency-modulated laser diode," Applied Optics, Vol. 25, No. 9, pp. 1439-1442 (1986)..

Beiser, L., "Holographic Scanning," Wiley (1988).

Boone, B.G., Bokulic, R.S., Andrews, G.B., McNutt, Jr., R.L., Dagalakis, N., "Optical and Microwave Communications System Conceptual Design for a Realistic Interstellar Explorer," 47th SPIE Meeting, Seattle, WA., July 2002.

DLP A Texas InstrumentsTechnology, http://www.dlp.com/dlp_technology/Default.asp?bhcp=1 (2004).

Gottlieb, M., Ireland, C.L., Ley, J.M., "Electro-Optic and Acousto-Optic Scanning and Deflection," Marcel Dekker Publisher (1983).

Langer, D., Mettenleiter, M., Hartl, F., Frohlich, C., "Imaging ladar for 3-D surveying of real world environments," International Journal of Robotic Research, Vol. 19, No. 11, pp. 1075-1088, (2000).

Lucent Technologies, "LambdaRouterTM All Optical Switch," http://www.lucent.com/pressroom/lambda.html (2004).

Marshall, G.F., "Optical Scanning," Marcel Dekker Publisher (1991).

McCarthy, D.C., "Photonic Switches: Fast, but Functional?," Photonics Spectra, March 2001. 
MEMS Optical, "Scanning Two Axis Tilt Mirrors," http://www.memsoptical.com/prodserv/products/twotiltmir.htm (2004).

Physik Instruments, http://www.physikinstrumente.de/products/prdetail.php?secid=3-12 (2004).

Sandia National Laboratories, "Sandia micromirrors may be part of Next Generation Space Telescope," http://www.sandia.gov/media/NewsRel/NR1999/space.htm (2004)

Spectra Switch Inc., http://www.spectraswitch.com/products/product_line/wavewalker_1x2_datasheet.htm (2004)

STAB, http://www.darpa.mil/mto/stab/ (2000).

Stann, B. L., Ruff, W. C., Sztankay, Z. G., "Intensity-modulated diode laser radar using frequency modulation/continuous wave ranging techniques," Optical Engineering, Vol. 35, No. 11, pp. 3270-3278 (1996).

Stone, W.C., "Three Degree-of-Freedom Telescoping Geometry Scanner, U.S. Patent Number 6,600,553, July 29, 2003.

Texas Instruments, "Digital Micromirror Device Delivering on Promises of "Brighter" Future for Imaging Applications,"

http://www.ti.com/corp/docs/company/history/dmd.shtml (2004).

Trellis Photonics,

http://www.techonline.com/community/member_company/non_member/2713 (2004).

Wallace, A. M., Buller, G. S., Walker, A. C., "3D imaging and ranging by time-correlated single photon counting," Journal of Computing and Control Engineering, pp. 157168, August 2001.

Younse, J., "Projection Display Systems Based on the Digital Micromirror Device," Proceedings of Micromechanical structures and Microelectromechanical Devices for Optical Processing and Multimedia Applications, p. 64, October 1995. 


\section{5 $\underline{\mathrm{ROIC} \text { and Focal Plane Array (FPA) References }}$}

Albota, M.A., et al, "Three-Dimensional Imaging Laser Radars with Geiger-Mode Avalanche Photodiode Arrays," Lincoln Laboratory Journal, Vol. 13, Num. 2, pp. 351367 (2002).

Anderson, C., et al., "HgCdTe APD Arrays and Readouts for 3D Imaging LADAR," Proceedings SPIE AeroSense (2002).

Aull, B., et al., "Geiger-Mode Avalanche Photodiodes for Three-Dimensional Imaging," Lincoln Laboratory Journal, Vol. 13, Num. 2, pp. 335-348 (2002).

Carson, J., "Infrared mosaic technology," Proceedings of the SPIE 62, 3-6 (1975).

Carson, J., "Infrared mosaic technology," Proceedings of the SPIE 510, 79-81 (1984).

Crowe, D.G., Norton, P.R., and Limperis, J.M., Detectors, Vol. 3 (Electro-Optical Components), Chapter 4, The Infrared and Electro-Optical Systems Handbook, William D. Rogatto, Editor, SPIE Optical Engineering Press, Bellingham, Washington, USA (1996).

Jack, M., et al., "HgCdTe APD Arrays, Readouts, and SCA's for 3D Imaging LADAR," Proceedings SPIE AeroSense (2003).

Ludwig, D., et al., "Identifying Targets under Trees - Jigsaw 3D-LADAR CFT Results," pre-print of paper to appear in SPIE AeroSense conference proceedings (2004).

Proceedings of the SPIE 1097, 930, and 1339 contain more than a dozen articles on Z technology.

Shaefer, T.T., "The RM 20B mosaic measurement experiment," Proceedings of the SPIE 62, 36-45 (1975).

Vampola, J.L., Readout Electronics for Infrared Sensors, Vol. 3 (Electro-Optical Components), Chapter 4, The Infrared and Electro-Optical Systems Handbook, William D. Rogatto, Editor, SPIE Optical Engineering Press, Bellingham, Washington, USA (1996).

\subsection{Super Resolution References}

Stoker, C.R., et al., "Super-Resolved Surface Reconstruction from Multiple Images," NASA Technical Report FIA-94-12, 1994. Analyzing Pathfinder Data using Virtual Reality and Super-resolved Imaging. Journal of Geophysical Research, February 1998. 


\subsection{Femtosecond Laser References}

Fermann, M.E., Yang, L.M., Stock, M.L., and Andrejco, M.J.: 'Environmentally Stable Kerr-Type Modelocked Erbium Fiber Laser Producing 300 fsec Pulses', Opt. Lett., vol. 19, pp. 43-45 (1994).

Fermann, M.E., Galvanauskas, A. and Harter, D.: 'All fiber source of $100 \mathrm{~nJ}$ subpicosecond pulses, Appl. Phys. Lett., vol. 64, pp. 1315-1317 (1994).

\subsection{Atmospheric Effects}

Andrews, L.C. and Phillips, R. L., "Laser beam propagation through random media" , SPIE Optical Engineering Press, Bellingham, WA. (1998).

Böckem B., Flach P., Weiss A., and Hennes M., "Refraction influence analysis and investigations on automated elimination of refraction effects on geodedtic measurements", XVI IMEKO World Congress 2000, 25-28, Vienna, September 2000.

Burton, R.R., Schott, and J.R., Brown, S.D., "Elastic LADAR Modeling for Synthetic Imaging Applications", SPIE Proceedings, Vol. 4816, pp. 144-155, July 2002.

Ciddor, P. E., "Refractive index of air: new equations for the visible and near infrared," Appl. Optics 35, 1566-1573 (1996).

Fasching, M. and Hauser, H., "Measurement of small angles between electromagnetic waves for refraction studies" Measurement Science Technology 4 ,1065-1069 (1993).

Flach, P., "Analysis of refraction influences in geodesy using image processing and turbulence models", Dissertation for Swiss Federal Institute of Technology (Diss. ETH No. 13844), Zurich (2000).

Flach P.H. and Maas H.G., "Vision-based techniques for refraction analysis in applications of terrestial geodesy" International Archives of Photogrammetry and Remote Sensing (IAPRS), Onuma, Vol. XXXII, Part. 5-3W12, pp. 195-201 (1999).

Gustavsson, T. and Bogren, J., "Measurement of Road Climatological Variables" Road Climate Center, Physical Geography, Department of Earth Sciences, University of Gotenborg, Box 460, 40530 Gotenborg, Sweenden. Presented at 11th SIRWEC, January 2002.

Hoph, F.A., Tomita, A., and Al-Jumaily G., "Second Harmonic Interferometers" Optics Letters 5, 386-88 (1980).

Huggett, G.R. and Slater, L.E., "Precision Electromagnetic Distance-Measuring 
Instrument for determining secular strain and fault movement" , In: N. Pavoni and R. Green Editors, Recent Crustal Movements, Tectonophysics 29 (1-4): 19-27 (1975).

Huiser, A. M. J., and Gachter, B.F., "A solution to atmospherically induced problems in very high-accuracy alignment and levelling" J. Phys D: Appl Phys, 22 pp1630-1638 (1989).

Ishida, A., "Two-wavelength displacement-measuring interferometer using secondharmonic light to Eliminate air-turbulence-induced errors", Japanese Journal of Appl Physics, vol 28, ppL473-L475 (1989).

Langbein, J. O., Linker, M. F., McGarr, A.F., and Slater, L.E., Precision of two-color geodimeter measurements: results from 15 months of observations" J Geophys Res 92(B11), 11644-11656 (1987).

Lassila, A. and Korpelainen, V., "An acoustic method for determination of the effective temperature and refractive index of air", SPIE Proceedings 5190, Recent Developments in Traceable Dimensional Measurements II, San Diego, August 3-8 2003, 316-326 (2003).

Levine, J., "Multiple wavelength electromagnetic distance measurements " in "Geodetic Refaraction" [Brunner FK editor, Springer, Berlin Heidelberg New York], 4551 (1984).

Lis, S. A., "Air-turbulence-compensated interferometer for IC manufacturing", SPIE Proceedings Vol. 2440, (Santa Clara meeting, 02/19 - 02/24/95) pp.891-901 (1995).

Minoshima, K. and Matsumoto H., "High-accuracy measurement of a 240-m distance in an optical tunnel by use of a compact femtosecond laser" Applied Optics 39 (30) pp 5512-5517 (2000).

Mihaljov, L., Hemming, B., Lehto, H., and Lassila, A., "A new method for improving the accuracy of measurements made with laser interferometer" (unpublished, 2001).

Rüeger, J.M., "Electronic Distance Measurement, an Introduction" Fourth Edition, Springer-Verlag, Berlin (1996).

Shirley, L., United States Patent No. US 6,229,619 B1, May 82001.

Williams, D.C., and Kahmen H., "Two wavelength angular refraction measurement", in "Geodetic Refaraction," F.K. Brunner, Editor, Springer, Berlin Heidelberg NY, 7-31. 
Appendix A

\title{
NIST Survey of LADAR Technology 2004
}

\author{
Contact: \\ Dr. William C. Stone \\ (301) 975-6075 \\ william.stone@nist.gov
}




\section{Commercial Systems}




\begin{tabular}{l|c} 
Manufacturer's Name & Z+F USA, Inc. \\
\hline Manufacturer's Address & $\begin{array}{c}\text { One Library Place, \#203, } \\
\text { Pittsburgh, PA 15110 }\end{array}$ \\
\hline Manufacturer's Phone & $(412) 469-9210$ \\
\hline Manufacturers Email & info@zf-usa.com \\
\hline Manufacturer's FAX & $(412) 469-9211$ \\
Name of Product & IMAGER \\
\hline Product Model Number & 5003 \\
\hline Year First Introduced & 2002
\end{tabular}

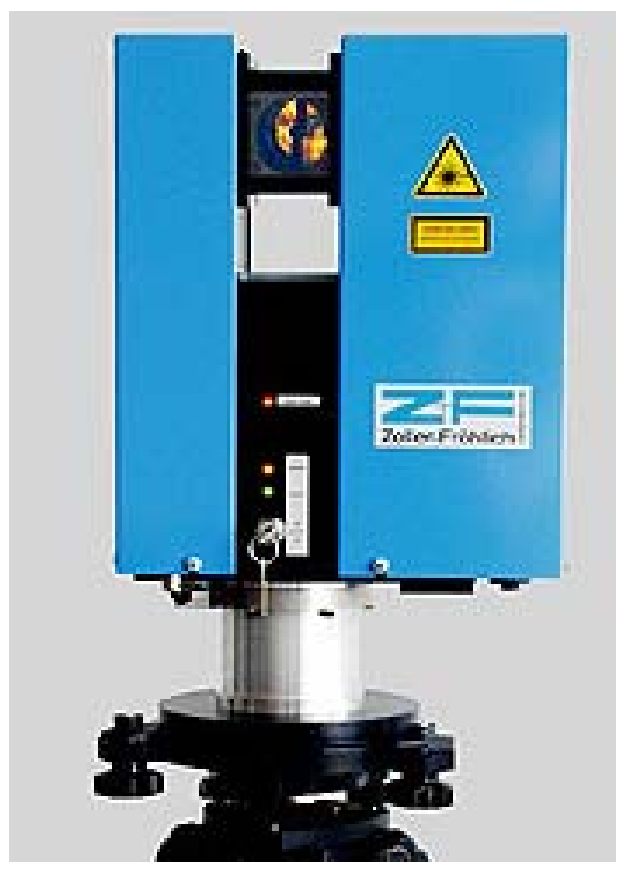

System Architecture

dual-frequency AMCW,

\begin{tabular}{l|c|c} 
& planar-mirror / pan scanning mechanism & \\
\hline Wavelength of illumination source & 780 & nanometers \\
\hline Illumination power & 22 & milliWatts \\
Beam diameter at Exit & 3.5 & millimeters \\
\hline Beam divergence angle & 0.1 & milli-radians \\
Sensor field of view, horizontal & & degrees \\
\hline Sensor field of view, vertical & 0 to 360 & degrees \\
\hline Maximum Range & -135 to +135 & meters
\end{tabular}

Frame rate (if FPA)

Array size (if FPA)

Pixel rate (if scanner or other)

Range resolution (depth)

Angular resolution

Range accuracy (depth) [note: if the accuracy varies with range, please list examples at several ranges]

\begin{tabular}{l|c|c} 
& $\mathrm{n} / \mathrm{a}$ & frames/second \\
\hline & $\mathrm{n} / \mathrm{a}$ & pixels \\
\hline & 625,000 & pixels/second \\
\hline please list & 0.38 & degrees \\
\hline idth, depth) & 0.01 & \\
or, including & 3 & millimeters \\
& $300 \times 350 \times 180$ & millimeters \\
\hline ired & & \\
& 16 & kilograms \\
\hline & 10 & U.S. $\$$ \\
\hline
\end{tabular}

Overall size of unit (height, width, depth) Weight of the complete sensor, including power supplies and any required interfaces

Retail cost

Lead time to delivery

10 


\section{NIST 2004 LADAR TECHNOLOGY SURVEY}

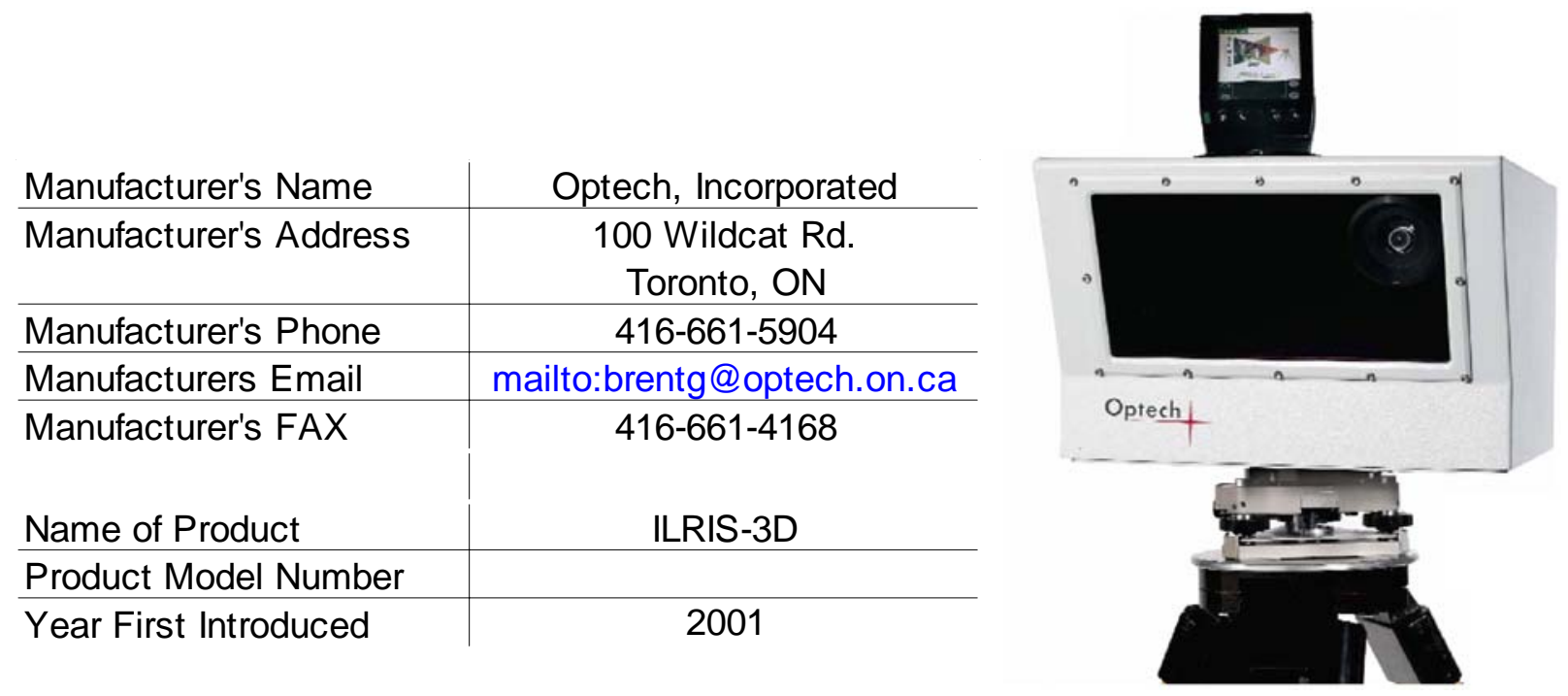

\begin{tabular}{l|c|c} 
System Architecture & Dual-axis orthogonal scanning mirror & \\
\hline Wavelength of illumination source & 1540 & nanometers \\
\hline Illumination power & $<10$ & milliWatts
\end{tabular}

Beam diameter at Exit Beam divergence angle

Sensor field of view, horizontal Sensor field of view, vertical Maximum Range

Frame rate (if FPA)

Array size (if FPA)

Pixel rate (if scanner or other)

Range resolution (depth)

Angular resolution

Range accuracy (depth) [note: if the accuracy varies with range, please list examples at several ranges]
12

0.17

40

40
millimeters milli-radians

degrees degrees meters

Overall size of unit (height, width, depth) Weight of the complete sensor, including power supplies and any required interfaces

Retail cost Lead time to delivery

\begin{tabular}{|c|c}
$\mathrm{N} / \mathrm{A}$ & frames/second \\
\hline $\mathrm{N} / \mathrm{A}$ & pixels \\
\hline 2000 & pixels/second \\
\hline $3 \mathrm{~mm}$ & millimeters \\
\hline $0.0015 \mathrm{deg}(26 \mu \mathrm{rad})$ & degrees \\
\hline & \\
10 & millimeters
\end{tabular}




\section{NIST 2004 LADAR TECHNOLOGY SURVEY}

\begin{tabular}{l|c} 
Manufacturer's Name & 3rdTech, Inc. \\
\hline Manufacturer's Address & $\begin{array}{c}\text { 119 E. Franklin St., 3rd } \\
\text { Fl., Chapel Hill, NC 27514 }\end{array}$ \\
\hline Manufacturer's Phone & (919) 929-1903 \\
\hline Manufacturers Email & info@3rdtech.com \\
\hline Manufacturer's FAX & $919-929-2098$ \\
Name of Product & DeltaSphere \\
\hline Product Model Number & 3000 \\
\hline Year First Introduced & 2000
\end{tabular}

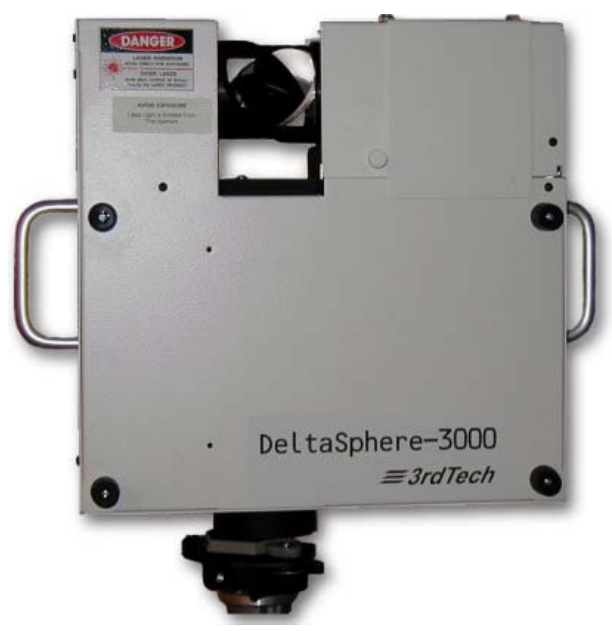

System Architecture

Scanner uses a rotating mirror for elevation and a rotating base for azimuth. The laser is an amplitude modulated continuous wave rangefinder "time of flight".

Wavelength of illumination source Illumination power

Beam diameter at Exit Beam divergence angle

Sensor field of view, horizontal Sensor field of view, vertical Maximum Range

Frame rate (if FPA)

Array size (if FPA)

Pixel rate (if scanner or other)

Range resolution (depth)

Angular resolution Range accuracy (depth) [note: if the accuracy varies with range, please list examples at several ranges]

"time of flight".

Overall size of unit (height, width, depth) Weight of the complete sensor, including power supplies and any required interfaces

Retail cost Lead time to delivery

$\$ 44,900 ; \$ 10,000$ for color option 30-45 nanometers

milliWatts

millimeters

milli-radians

degrees degrees meters

\begin{tabular}{|c|c}
\hline to +90 & degrees \\
\hline 12 & meters
\end{tabular}




\section{NIST 2004 LADAR TECHNOLOGY SURVEY}

\begin{tabular}{l|c|} 
Manufacturer's Name & \begin{tabular}{c} 
MetricVision, Inc. \\
\hline Manufacturer's Address
\end{tabular} \\
$\begin{array}{l}\text { 8500 Cinder Bed Rd, } \\
\text { Newington, VA 22122 }\end{array}$ \\
\hline Manufacturer's Phone & $(703) 550-2945$ \\
\hline Manufacturers Email & sales@metricvision.com \\
\hline Manufacturer's FAX & (703) 550-2949 \\
Name of Product & FM Coherent Laser Radar \\
\hline Product Model Number & MV200 \\
\hline Year First Introduced & 2001
\end{tabular}

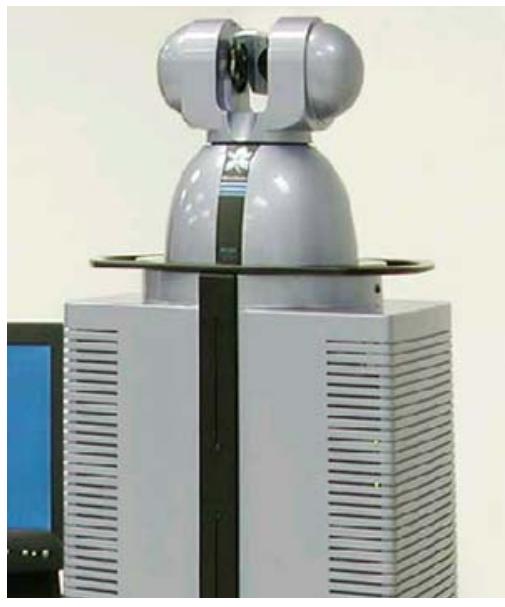

System Architecture

Frequency Modulated (Chirped) Coherent Laser Radar Ranging accurate to 2.5 parts per million up to 60 meters. The laser beam is expanded to $40 \mathrm{~mm}$ and then focused to a diffraction limited spot whose size is approx. 100 microns plus 60 microns per meter. Th

Wavelength of illumination source Illumination power 2 (US, International Class 1 eye-safe) nanometers

\begin{tabular}{l|c|c} 
& & Beam diameter at Exit \\
\hline Beam divergence angle & Focused beam 0.16mm @ 1meter & millimeters \\
\hline Sensor field of view, horizontal & $+/-200$ (i.e. full 360) & milli-radians \\
\hline Sensor field of view, vertical & $+/-45$ & degrees \\
\hline Maximum Range & 60 & degrees \\
\hline
\end{tabular}

Frame rate (if FPA)

Array size (if FPA) \begin{tabular}{l|l} 
NA & frames/second
\end{tabular}

Pixel rate (if scanner or other)

Range resolution (depth)

Angular resolution

Range accuracy (depth) [note: if the accuracy varies with range, please list examples at several ranges]

NA
pixels

1000

0.0001
pixels/second millimeters degrees

0.000002

2.5 parts per million: $0.025 \mathrm{~mm} @ 10 \mathrm{~m}$, $0.065 \mathrm{~mm}$ at $25 \mathrm{~m}, 0.15 \mathrm{~mm}$ at $60 \mathrm{~m}$ millimeters

Overall size of unit (height, width, depth) Weight of the complete sensor, including power supplies and any required interfaces kilograms

Retail cost Lead time to delivery $324 \times 454 \times 823$ millimeters

\begin{tabular}{|c|c|}
\hline $324 \times 454 \times 823$ & millimeters \\
\hline Sensor $=40$, Cart $=80$ & kilograms \\
\hline $\begin{array}{c}\$ 320,000 \text { for } 24 \text { meter, } \$ 410,000 \text { for } 60 \\
\text { meter }\end{array}$ & U.S. $\$$ \\
\hline 12 & weeks \\
\hline
\end{tabular}




\section{NIST 2004 LADAR TECHNOLOGY SURVEY}

\begin{tabular}{l|l} 
Source Name & PMDTec/S-TEC GmbH \\
\hline Source Address & $\begin{array}{l}\text { Wilhelm-von-Humboldt- } \\
\text { Platz 13, 57076 } \\
\text { Siegen/Germay }\end{array}$ \\
\hline Source Contact Phone & (49) 271-2385380 \\
\hline Source Email & info@PMDTec.com \\
\hline Source FAX & (49) 271-2385389 \\
Name of Product & $\begin{array}{l}\text { PMD-"LADAR" } \\
\text { Prototypes-xD }\end{array}$ \\
\hline Product Model Number & \\
\hline Year First Introduced & 1999
\end{tabular}

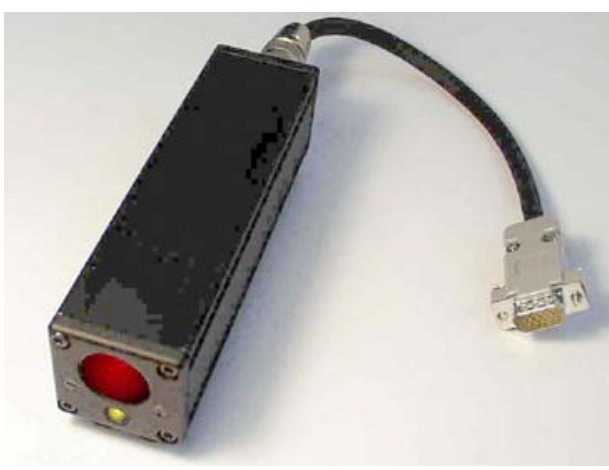

System Architecture

Focal plane Arrays based on PMD elements with various number and orientation of PMD pixels, various technologies, e.g. pure CMOS or hybrids (CMOS readout combined with GaAs PMD-Front-Ends), integrated in compact housings with standard interfaces (RS 232 ,

Wavelength of illumination source depends on application, e.g. $658 \mathrm{~nm}$ Illumination power laser or $890 / 850 \mathrm{~nm}$ LEDs-arrays

\begin{tabular}{l|c|c} 
Illumination power & from $1 \mathrm{~mW}$ laser up to $1 \mathrm{~W}$ LED power & \\
Beam diameter at Exit & & millimeters \\
\hline Beam divergence angle & $0.1^{\circ}$ for $1 \mathrm{D}-$ depth \\
& $45^{\circ}$ for 3D illumination & degrees \\
Sensor field of view, horizontal & e.g. $45^{\circ}$ for 3D application & degrees \\
\hline Sensor field of view, vertical & e.g. $16^{\circ}$ for 3D application & degrees \\
\hline Maximum Range & 15 & meters
\end{tabular}

\begin{tabular}{l|c|c} 
Frame rate (if FPA) & $\begin{array}{c}1 \text { to } 100 \text { on natural surfaces, up to } 1000 \\
\text { on reflector }\end{array}$ & frames/second \\
\hline $\begin{array}{l}\text { Array size (if FPA) } \\
\text { Pixel rate (if scanner or other) }\end{array}$ & $\begin{array}{c}\text { single elements, line sensors, arrays up } \\
\text { to } 16 \times 16 \text {, in } 2003 \text { up to } 64 \times 16\end{array}$ & pixels \\
\hline $\begin{array}{l}\text { Range resolution (depth) } \\
\text { Angular resolution }\end{array}$ & $\begin{array}{c}\text { depends on measurement conditions: } \\
5 \text { to } 200 \mathrm{~mm}\end{array}$ & millimeters \\
\hline $\begin{array}{l}\text { Range accuracy (depth) [note: if the } \\
\text { accuracy varies with range, please list } \\
\text { examples at several ranges; accuracy } \\
\text { should be reported at the } 1 \text {-sigma level } \\
\text { standard deviation about the true range; if } \\
\text { data are available at } 2 \text { and } 3 \text { sigma, } \\
\text { please provide those data] }\end{array}$ & depend on specified FOV & degrees \\
$\begin{array}{l}\text { Overall size of unit (height, width, depth) } \\
\begin{array}{l}\text { power supplies and any required } \\
\text { interfaces }\end{array}\end{array}$ & $\begin{array}{l}161 \times 38.5 \times 38.5 \\
\begin{array}{l}\text { Retail cost or Estimated cost to duplicate } \\
\text { a lab prototype. }\end{array}\end{array}$ & millimeters \\
$\begin{array}{l}\text { Lead time to delivery (or estimated time to } \\
\text { duplicate a lab prototype) }\end{array}$ & 0.3 & millimeters \\
\hline
\end{tabular}




\section{NIST 2004 LADAR TECHNOLOGY SURVEY}

\begin{tabular}{l|l} 
Source Name & $\begin{array}{l}\text { CSEM, Swiss Center for } \\
\text { Electronics and } \\
\text { Microtechnology }\end{array}$ \\
\hline Source Address & $\begin{array}{l}\text { Badenerstrasse 569, CH- } \\
8048 \text { Zurich, Switzerland }\end{array}$ \\
\hline Source Contact Phone & +4114971447 \\
\hline Source Email & imaging@csem.ch \\
\hline Source FAX & +4114971400 \\
Name of Device/Product & Swiss Ranger \\
\hline $\begin{array}{l}\text { Identification (e.g. model, } \\
\text { make) }\end{array}$ & SR-2 \\
\hline
\end{tabular}

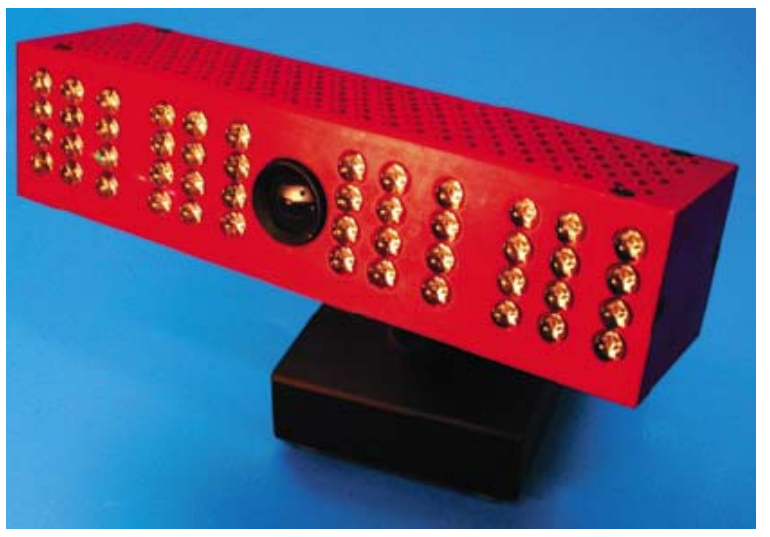

\begin{tabular}{|c|c|c|}
\hline System Architecture & $\begin{array}{c}\text { Focal Plane Array with custom } \\
\text { CMOS/CCD demodulation pixel image } \\
\text { sensor. Miniature 3D time-of-flight } \\
\text { camera with AM-CW LED illumination. } \\
\text { Read-out and programming via standard } \\
\text { USB interface. }\end{array}$ & \\
\hline Wavelength of illumination source & 820 & nanometers \\
\hline Illumination power & 800 & milliWatts \\
\hline Beam diameter at Exit & 100 & millimeters \\
\hline Beam divergence angle & 0.7 & milli-radians \\
\hline Sensor field of view, horizontal & $+/-15$ & degrees \\
\hline Sensor field of view, vertical & $+/-15$ & degrees \\
\hline Maximum Range & 20 & meters \\
\hline Frame rate (if FPA) & 30 & frames/second \\
\hline Array size (if FPA) & $128 \times 160$ & pixels \\
\hline Pixel rate (if scanner or other) & & pixels/second \\
\hline Range resolution (depth) & 1 & millimeters \\
\hline Angular resolution & 0.5 & degrees \\
\hline $\begin{array}{l}\text { Range accuracy (depth) [note: if the } \\
\text { accuracy varies with range, please list } \\
\text { examples at several ranges; accuracy } \\
\text { should be reported at the } 1 \text {-sigma level } \\
\text { standard deviation about the true range; if } \\
\text { data are available at } 2 \text { and } 3 \text { sigma, } \\
\text { please provide those data] }\end{array}$ & 5 & millimeters \\
\hline Overall size of unit (height, width, depth) & $40 \times 125 \times 30$ & millimeters \\
\hline $\begin{array}{l}\text { Weight of the complete sensor, including } \\
\text { power supplies and any required } \\
\text { interfaces }\end{array}$ & 0.3 & kilograms \\
\hline $\begin{array}{l}\text { Retail cost or Estimated cost to duplicate } \\
\text { a lab prototype. }\end{array}$ & $\$ 4,600$ & U.S. $\$$ \\
\hline $\begin{array}{l}\text { Lead time to delivery (or estimated time to } \\
\text { duplicate a lab prototype) }\end{array}$ & 8 & weeks \\
\hline
\end{tabular}




\section{NIST 2004 LADAR TECHNOLOGY SURVEY}

\begin{tabular}{l|l} 
Source Name & $\begin{array}{l}\text { Riegl Laser Measurement } \\
\text { System }\end{array}$ \\
\hline Source Address & $\begin{array}{l}7081 \text { Grand National Drive, } \\
\text { Suite 112 Orlando, Florida } \\
32819\end{array}$ \\
\hline Source Contact Phone & $407-248-9927$ \\
\hline Source Email & info@rieglusa.com \\
\hline Source FAX & 4072482636 \\
Name of Device/Product & $\begin{array}{l}\text { LPM 25HA-C - LIDAR } \\
\text { Scanner }\end{array}$ \\
\hline $\begin{array}{l}\text { Identification (e.g. model, } \\
\text { make) }\end{array}$ & \\
\hline Year First Introduced & 2001
\end{tabular}

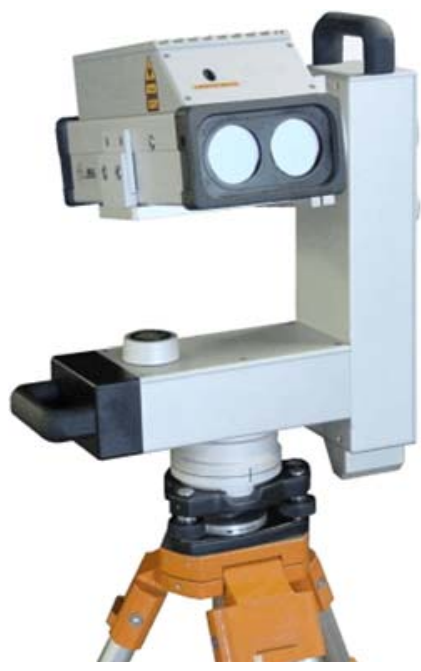

\begin{tabular}{|c|c|c|}
\hline System Architecture & $\begin{array}{l}\text { Scanner Pan \& Tilt. pure pulse time-of- } \\
\text { flight }\end{array}$ & \\
\hline Wavelength of illumination source & Near Infrared & nanometers \\
\hline Laser Class & Class 1 EyeSafe & \\
\hline Beam diameter at Exit & $n / a$ & \\
\hline Beam divergence & $\begin{array}{l}15 \mathrm{~mm} @ 10 \mathrm{~m} \\
20 \mathrm{~mm} @ 20 \mathrm{~m} \\
60 \mathrm{~mm} @ 60 \mathrm{~m}\end{array}$ & \\
\hline Sensor field of view, horizontal & 0 to 360 & degrees \\
\hline Sensor field of view, vertical & 0 to 360 & degrees \\
\hline Maximum Range & 100 & meters \\
\hline Frame rate (if FPA) & $\mathrm{n} / \mathrm{a}$ & frames/second \\
\hline Array size (if FPA) & $\mathrm{n} / \mathrm{a}$ & pixels \\
\hline Pixel Rate (if scanner or other) & 1000 & pixels/second \\
\hline Range resolution (depth) & $1 \mathrm{~mm}$ & millimeters \\
\hline Angular resolution & 0.009 & degrees \\
\hline Range accuracy & 4 to 8 & millimeters \\
\hline Length \& Diameter & $250 \times 300 \times 320$ & millimeters \\
\hline $\begin{array}{l}\text { Weight of the complete sensor, including } \\
\text { power supplies and any required } \\
\text { interfaces }\end{array}$ & 15 & kilograms \\
\hline $\begin{array}{l}\text { Retail cost or Estimated cost to duplicate } \\
\text { a lab prototype. }\end{array}$ & $\$ 75,000$ & U.S. $\$$ \\
\hline $\begin{array}{l}\text { Lead time to delivery (or estimated time to } \\
\text { duplicate a lab prototype) }\end{array}$ & 6 to 8 & weeks \\
\hline
\end{tabular}




\section{NIST 2004 LADAR TECHNOLOGY SURVEY}

\begin{tabular}{l|l} 
Source Name & $\begin{array}{l}\text { Riegl Laser Measurement } \\
\text { System }\end{array}$ \\
\hline Source Address & $\begin{array}{l}7081 \text { Grand National Drive, } \\
\text { Suite 112 Orlando, Florida } \\
32819\end{array}$ \\
\hline Source Contact Phone & $407-248-9927$ \\
\hline Source Email & info@rieglusa.com \\
\hline Source FAX & 4072482636 \\
Name of Device/Product & $\begin{array}{l}\text { LPM 800HA - LIDAR } \\
\text { Scanner }\end{array}$ \\
\hline $\begin{array}{l}\text { Identification (e.g. model, } \\
\text { make) }\end{array}$ & \\
\hline Year First Introduced & 2002
\end{tabular}

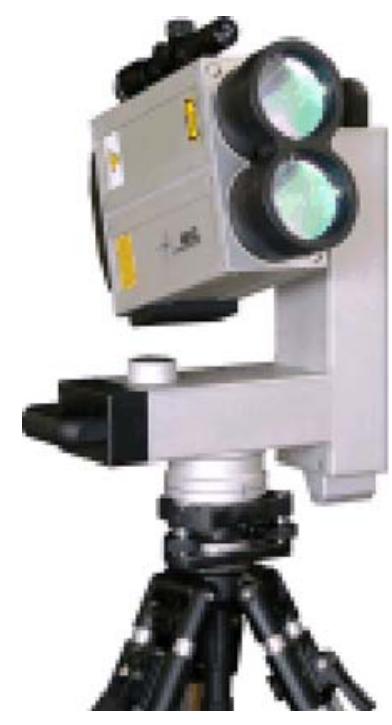

\begin{tabular}{|c|c|c|}
\hline System Architecture & $\begin{array}{l}\text { Scanner Pan \& Tilt. pure pulse time-of- } \\
\text { flight }\end{array}$ & \\
\hline Wavelength of illumination source & Near Infrared & nanometers \\
\hline Laser Class & Class 1 EyeSafe & \\
\hline Beam divergence & 1.3 & milliradian \\
\hline Sensor field of view, horizontal & 0 to 360 & degrees \\
\hline Sensor field of view, vertical & 0 to 360 & degrees \\
\hline Maximum Range & 800 & meters \\
\hline Frame rate (if FPA) & $\mathrm{n} / \mathrm{a}$ & frames/second \\
\hline Array size (if FPA) & $\mathrm{n} / \mathrm{a}$ & pixels \\
\hline Pixel rate (if scanner or other) & 1000 & pixels/second \\
\hline Range resolution (depth) & $1 \mathrm{~mm}$ & millimeters \\
\hline Angular resolution & 0.009 & degrees \\
\hline Range accuracy & 15 & millimeters \\
\hline Length \& Diameter & $287 \times 300 \times 320$ & millimeters \\
\hline $\begin{array}{l}\text { Weight of the complete sensor, including } \\
\text { power supplies and any required } \\
\text { interfaces }\end{array}$ & 15 & kilograms \\
\hline $\begin{array}{l}\text { Retail cost or Estimated cost to duplicate } \\
\text { a lab prototype. }\end{array}$ & $\$ 75,000$ & U.S. $\$$ \\
\hline $\begin{array}{l}\text { Lead time to delivery (or estimated time to } \\
\text { duplicate a lab prototype) }\end{array}$ & 6 to 8 & weeks \\
\hline
\end{tabular}




\section{NIST 2004 LADAR TECHNOLOGY SURVEY}

\begin{tabular}{l|l} 
Source Name & $\begin{array}{l}\text { Riegl Laser Measurement } \\
\text { System }\end{array}$ \\
\hline Source Address & $\begin{array}{l}7081 \text { Grand National Drive, } \\
\text { Suite 112 Orlando, Florida } \\
32819\end{array}$ \\
\hline $\begin{array}{l}\text { Source Contact Phone } \\
\text { S07-248-9927 } \\
\text { info@rieglusa.com }\end{array}$ \\
\hline $\begin{array}{l}\text { Source Email } \\
\text { Source FAX }\end{array}$ & 4072482636 \\
\hline $\begin{array}{l}\text { Name of Device/Product } \\
\text { LMS Z210 - LIDAR } \\
\text { Scanner }\end{array}$ \\
\hline $\begin{array}{l}\text { Identification (e.g. model, } \\
\text { make) }\end{array}$ & \\
\hline $\begin{array}{l}\text { Year First Introduced } \\
\text { Sel }\end{array}$ & 1998
\end{tabular}

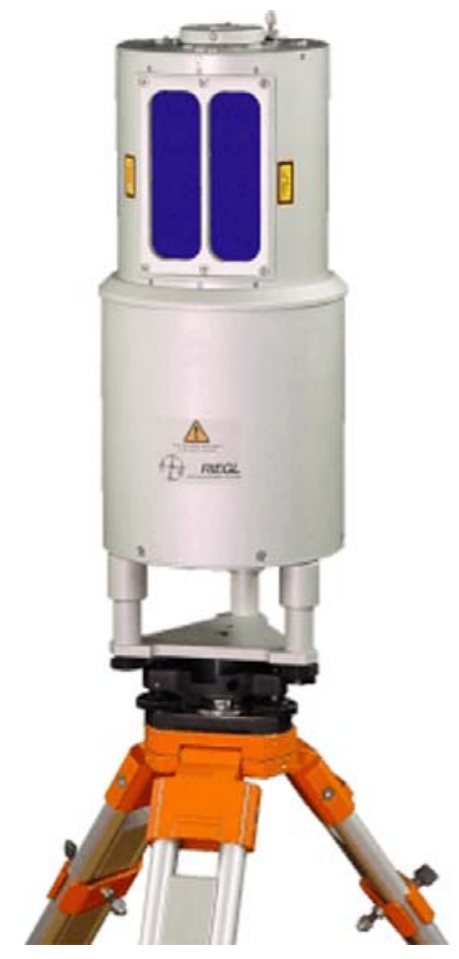

\begin{tabular}{l|c|c} 
System Architecture & $\begin{array}{c}\text { Scanner ( polygon-polygon) . Pure pulse } \\
\text { time-of-flight }\end{array}$ & \\
\hline Wavelength of illumination source & 904 & nanometers \\
\hline Laser Class & Class 1 Eye Safe & \\
\hline Beam divergence & 3 & milli-radians \\
Sensor field of view, horizontal & 0 to 330 & degrees \\
\hline Sensor field of view, vertical & 80 & degrees \\
\hline Maximum Range & 450 & meters
\end{tabular}

\begin{tabular}{l|c|c} 
Frame rate (if FPA) & n/a & frames/second \\
\hline Array size (if FPA) & n/a & pixels \\
\hline Pixel rate (if scanner or other) & 28,000 & pixels/second \\
\hline Range resolution (depth) & 0.18 & millimeters \\
\hline Angular resolution & 25 & degrees \\
\hline Range accuracy & $435 \times 210$ & millimeters \\
$\begin{array}{l}\text { Length \& Diameter } \\
\begin{array}{l}\text { Weight of the complete sensor, including } \\
\text { power supplies and any required } \\
\text { interfaces }\end{array}\end{array}$ & 15 & \\
\hline $\begin{array}{l}\text { Retail cost or Estimated cost to duplicate } \\
\text { a lab prototype. }\end{array}$ & & kilograms \\
\hline $\begin{array}{l}\text { Lead time to delivery (or estimated time to } \\
\text { duplicate a lab prototype) }\end{array}$ & $\$ 84,000$ & U.S. $\$$ \\
\hline
\end{tabular}




\section{NIST 2004 LADAR TECHNOLOGY SURVEY}

\begin{tabular}{l|l|} 
Source Name & $\begin{array}{l}\text { Riegl Laser Measurement } \\
\text { System }\end{array}$ \\
\hline Source Address & $\begin{array}{l}7081 \text { Grand National Drive, } \\
\text { Suite 112 Orlando, Florida } \\
32819\end{array}$ \\
\hline Source Contact Phone & $407-248-9927$ \\
\hline Source Email & info@rieglusa.com \\
\hline Source FAX & 4072482636 \\
\hline Name of Device/Product & $\begin{array}{l}\text { LMS Z360 - LIDAR } \\
\text { Scanner }\end{array}$ \\
\hline $\begin{array}{l}\text { Identification (e.g. model, } \\
\text { make) }\end{array}$ & \\
\hline Year First Introduced & 2002
\end{tabular}

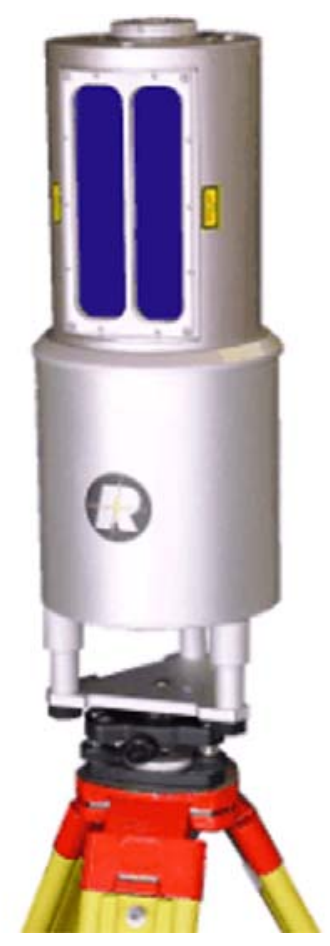

System Architecture

Scanner ( polygon-polygon) . pure pulse time-of-flight

Wavelength of illumination source 904 nanometers Laser Class Class 1 Eye Safe

Beam divergence

Sensor field of view, horizontal

Sensor field of view, vertical

Maximum Range

\begin{tabular}{l|l} 
& \\
\hline 2 & milli-radians
\end{tabular}

Frame rate (if FPA)

Array size (if FPA)

Pixel rate (if scanner or other)

Range resolution (depth)

Angular resolution

Range accuracy

Length \& Diameter

Weight of the complete sensor, including power supplies and any required interfaces

Retail cost or Estimated cost to duplicate a lab prototype.

Lead time to delivery (or estimated time to duplicate a lab prototype) 


\section{NIST 2004 LADAR TECHNOLOGY SURVEY}

\begin{tabular}{l|l|} 
Source Name & $\begin{array}{l}\text { Riegl Laser Measurement } \\
\text { System }\end{array}$ \\
\hline Source Address & $\begin{array}{l}7081 \text { Grand National Drive, } \\
\text { Suite 112 Orlando, Florida } \\
32819\end{array}$ \\
\hline Source Contact Phone & $407-248-9927$ \\
\hline Source Email & info@rieglusa.com \\
\hline Source FAX & 4072482636 \\
\hline Name of Device/Product & $\begin{array}{l}\text { LMS Z420 - LIDAR } \\
\text { Scanner }\end{array}$ \\
\hline $\begin{array}{l}\text { Identification (e.g. model, } \\
\text { make) }\end{array}$ & \\
\hline Year First Introduced & 2002
\end{tabular}

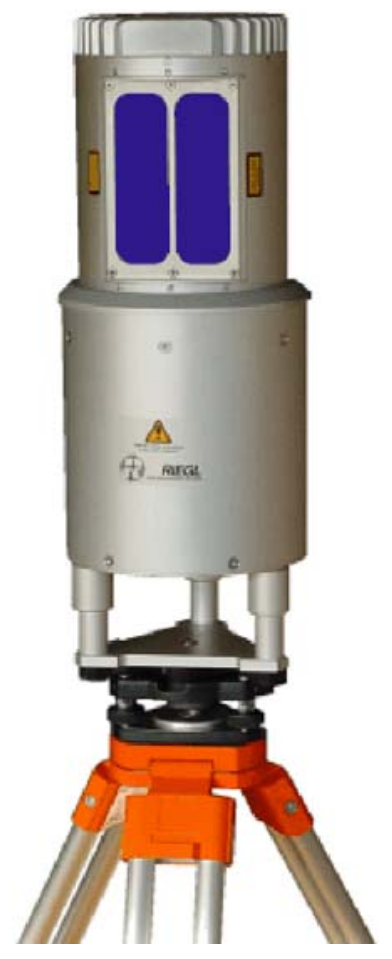

\begin{tabular}{|c|c|c|}
\hline System Architecture & $\begin{array}{c}\text { Scanner ( polygon-polygon) } \text {. pure pulse } \\
\text { time-of-flight }\end{array}$ & \\
\hline Wavelength of illumination source & Near Infrared & nanometers \\
\hline Laser Class & Class 3R & \\
\hline Beam divergence & 0.25 & milli-radians \\
\hline Sensor field of view, horizontal & 0 to 360 & degrees \\
\hline Sensor field of view, vertical & 0 to 90 & degrees \\
\hline Maximum Range & 1000 & meters \\
\hline Frame rate (if FPA) & $\mathrm{n} / \mathrm{a}$ & frames/second \\
\hline Array size (if FPA) & $\mathrm{n} / \mathrm{a}$ & pixels \\
\hline Pixel rate (if scanner or other) & 3,000 & pixels/second \\
\hline Range resolution (depth) & $5 \mathrm{~mm}$ & millimeters \\
\hline Angular resolution & 0.002 & degrees \\
\hline Range accuracy & 6 & millimeters \\
\hline Length \& Diameter & $463 \times 210$ & millimeters \\
\hline $\begin{array}{l}\text { Weight of the complete sensor, including } \\
\text { power supplies and any required } \\
\text { interfaces }\end{array}$ & 15 & kilograms \\
\hline $\begin{array}{l}\text { Retail cost or Estimated cost to duplicate } \\
\text { a lab prototype. }\end{array}$ & $\$ 150,000$ & U.S. $\$$ \\
\hline $\begin{array}{l}\text { Lead time to delivery (or estimated time to } \\
\text { duplicate a lab prototype) }\end{array}$ & 6 to 8 & weeks \\
\hline
\end{tabular}




\section{NIST 2004 LADAR TECHNOLOGY SURVEY}

\begin{tabular}{l|l} 
Source Name & MENSI, Inc. \\
\hline Source Address & $\begin{array}{l}\text { 35 Technology Parkway } \\
\text { South, Suite 170, } \\
\text { Norcross, GA 30092 }\end{array}$ \\
\hline Source Contact Phone & $(770) 613-5255$ \\
\hline Source Email & info@mensi.com \\
\hline Source FAX & $(770) 613-5254$ \\
Name of Device/Product & GS100 \\
\hline $\begin{array}{l}\text { Identification (e.g. model, } \\
\text { make) }\end{array}$ & n/a \\
\hline Year First Introduced & 2002
\end{tabular}

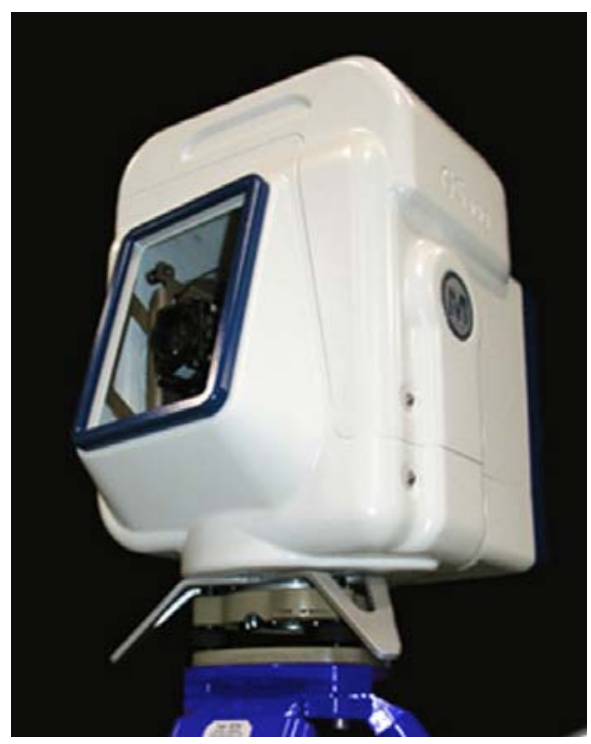

Wavelength of illumination source Illumination power 532

\begin{tabular}{l|c|c} 
Beam diameter at Exit & 12 & millimeters \\
\hline Beam divergence angle & Autofocusing system or adjustable focus \\
& distance & milli-radians \\
Sensor field of view, horizontal & 360 & degrees \\
\hline Sensor field of view, vertical & 60 & degrees \\
\hline Maximum Range & 100 & meters
\end{tabular}

Frame rate (if FPA) frames/second

Array size (if FPA) pixels

Pixel rate (if scanner or other)

Range resolution (depth)

Angular resolution

Range accuracy (depth) [note: if the accuracy varies with range, please list examples at several ranges; accuracy should be reported at the 1-sigma level standard deviation about the true range; if data are available at 2 and 3 sigma, please provide those data]

$1.5 \mathrm{~mm}$ at $25 \mathrm{~m}$ (4 shots averaging) $6 \mathrm{~mm}$ at $100 \mathrm{~m}$ (4 shots averaging) on $99 \%$ albedo target

0.1

0.0018
ixels/second millimeters degrees

millimeters

Overall size of unit (height, width, depth) Weight of the complete sensor, including power supplies and any required interfaces

Retail cost or Estimated cost to duplicate a lab prototype. Lead time to delivery (or estimated time to duplicate a lab prototype) millimeters $380 \times 420 \times 280$ 30 kilograms U.S. $\$$ $\$ 125,000$ Maximum 4 weeks 


\section{NIST 2004 LADAR TECHNOLOGY SURVEY}

\begin{tabular}{l|l} 
Source Name & MENSI, Inc. \\
\hline Source Address & $\begin{array}{l}\text { 35 Technology Parkway } \\
\text { South, Suite 170, } \\
\text { Norcross, GA 30092 }\end{array}$ \\
\hline Source Contact Phone & $(770)$ 613-5255 \\
\hline Source Email & info@mensi.com \\
\hline Source FAX & $(770)$ 613-5254 \\
Name of Device/Product & S10 \\
\hline $\begin{array}{l}\text { Identification (e.g. model, } \\
\text { make) }\end{array}$ & N/A \\
\hline Year First Introduced & 1992
\end{tabular}

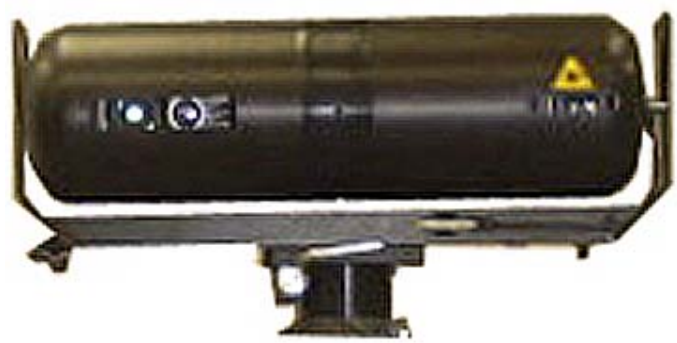

\begin{tabular}{l|c|c} 
Wavelength of illumination source & 632 & nanometers \\
\hline Illumination power & 2.5 & milliWatts \\
Beam diameter at Exit & 8 & millimeters \\
\hline Beam divergence angle & Autofocusing system & milli-radians \\
& & \\
Sensor field of view, horizontal & 320 & degrees \\
\hline Sensor field of view, vertical & 46 & degrees \\
\hline Maximum Range & 15 & meters
\end{tabular}

Frame rate (if FPA)

Array size (if FPA)

Pixel rate (if scanner or other)

Range resolution (depth)

Angular resolution

Range accuracy (depth) [note: if the accuracy varies with range, please list examples at several ranges; accuracy should be reported at the 1-sigma level standard deviation about the true range; if data are available at 2 and 3 sigma, please provide those data]

$0.35+0.01^{*} \mathrm{~d}^{\wedge} 2$ for

$\mathrm{d}=$ range in meters

millimeters

Overall size of unit (height, width, depth) Weight of the complete sensor, including power supplies and any required interfaces

Retail cost or Estimated cost to duplicate a lab prototype. Lead time to delivery (or estimated time to duplicate a lab prototype)

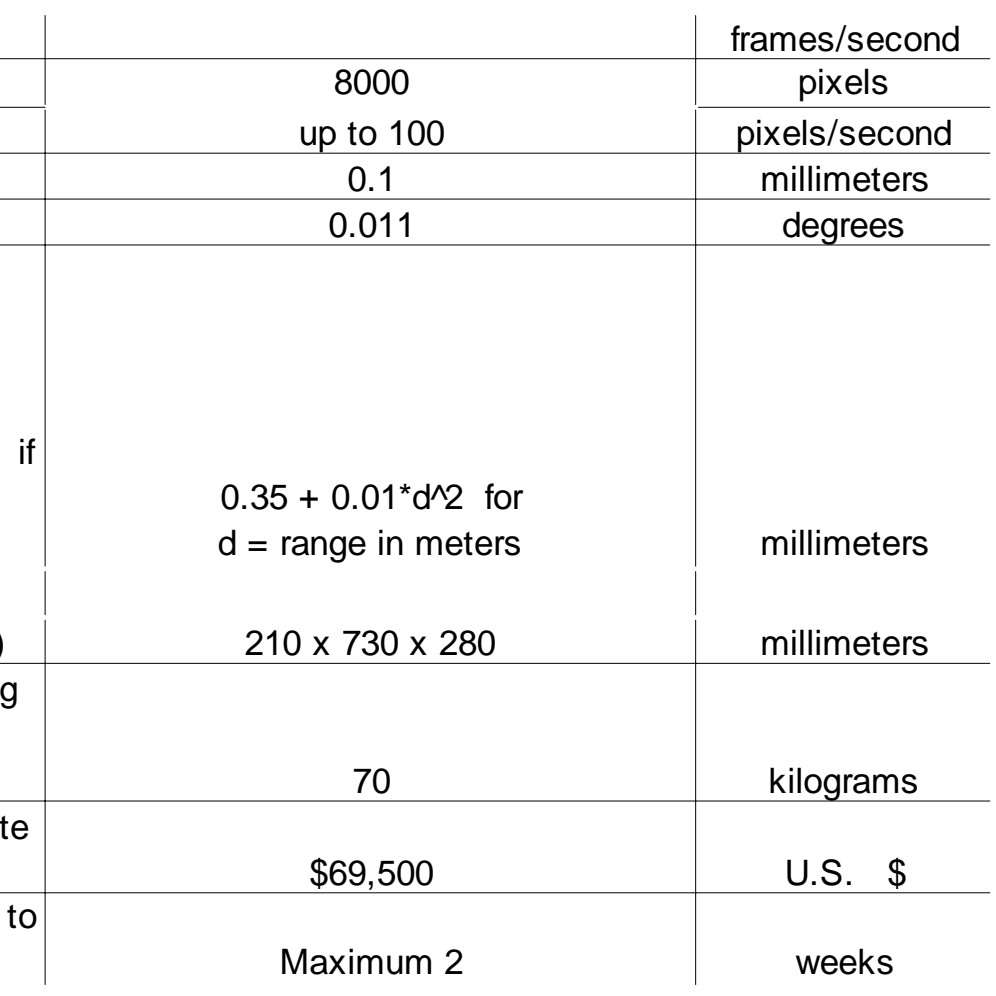




\section{NIST 2004 LADAR TECHNOLOGY SURVEY}

\begin{tabular}{l|l} 
Source Name & MENSI, Inc. \\
\hline Source Address & $\begin{array}{l}\text { 35 Technology Parkway } \\
\text { South, Suite 170, } \\
\text { Norcross, GA 30092 }\end{array}$ \\
\hline Source Contact Phone & $(770)$ 613-5255 \\
\hline Source Email & info@mensi.com \\
\hline Source FAX & $(770)$ 613-5254 \\
Name of Device/Product & S25 \\
\hline $\begin{array}{l}\text { Identification (e.g. model, } \\
\text { make) }\end{array}$ & N/A \\
\hline Year First Introduced & 1992
\end{tabular}

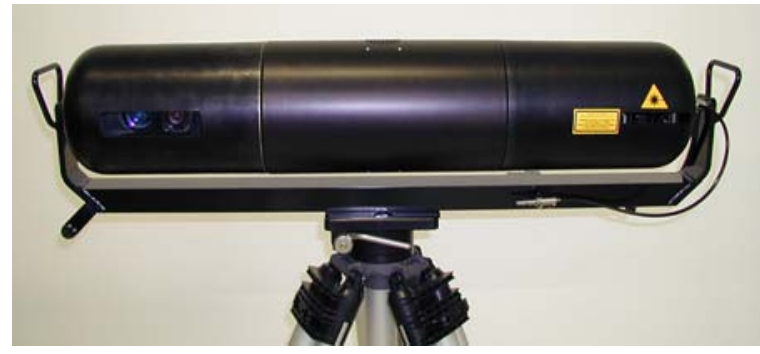

Wavelength of illumination source Illumination power

Beam diameter at Exit

Beam divergence angle

Sensor field of view, horizontal

Sensor field of view, vertical

Maximum Range

\begin{tabular}{|c|c}
632 & nanometers \\
\hline 2.5 & milliWatts \\
& millimeters \\
\hline Autofocusing system & milli-radians \\
& \\
320 & degrees \\
\hline 46 & degrees \\
\hline 35 & meters
\end{tabular}

Frame rate (if $\mathrm{FPA}$ )

Array size (if FPA)

Pixel rate (if scanner or other)

Range resolution (depth)

Angular resolution

Range accuracy (depth) [note: if the accuracy varies with range, please list examples at several ranges; accuracy should be reported at the 1-sigma level standard deviation about the true range; if data are available at 2 and 3 sigma, please provide those data]

$0.35+0.01^{*} \mathrm{~d}^{\wedge} 2 \mathrm{~mm}$ at range $\mathrm{d}$ in meters

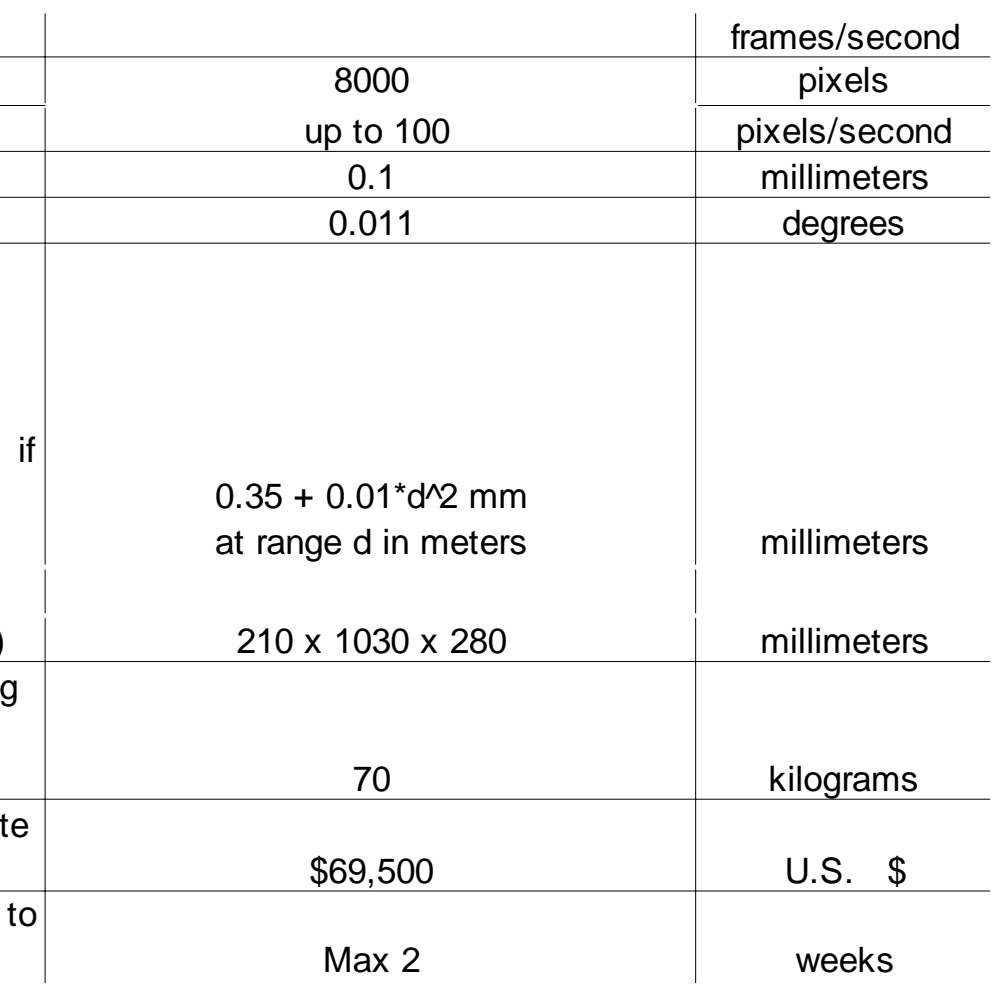

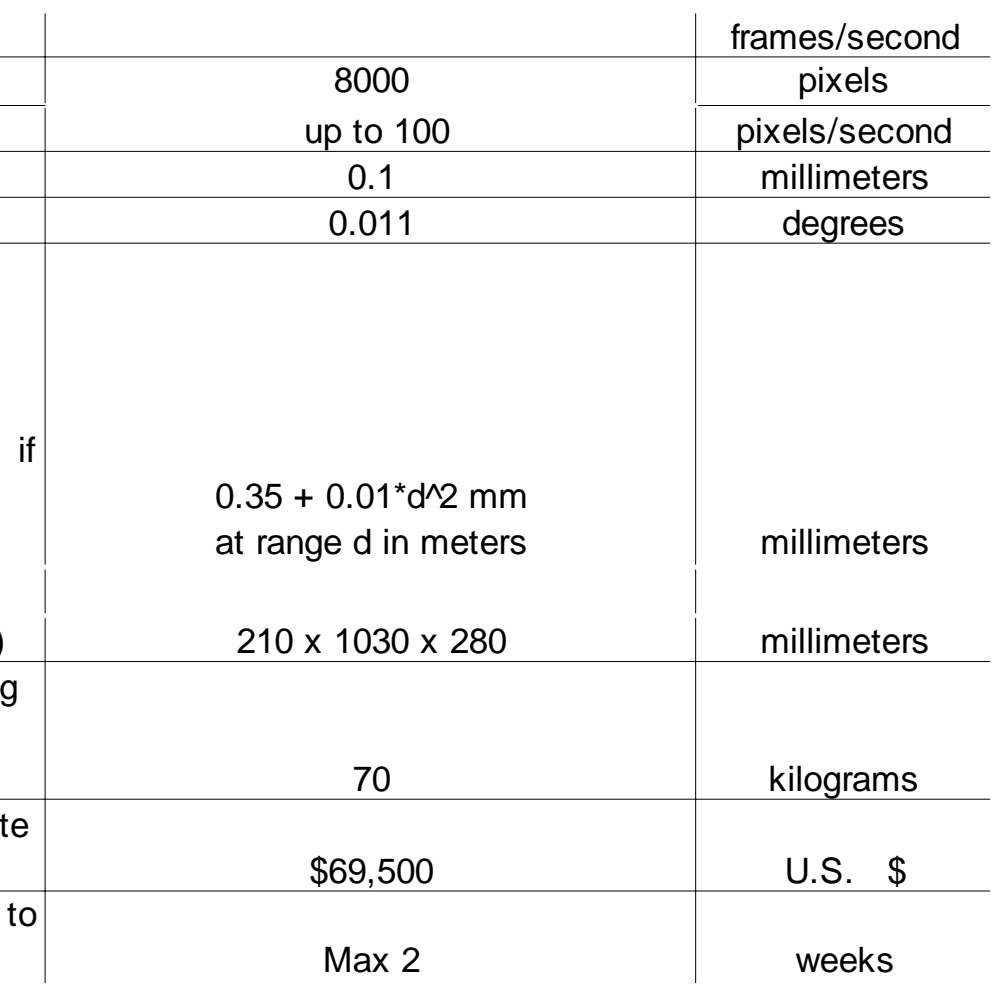

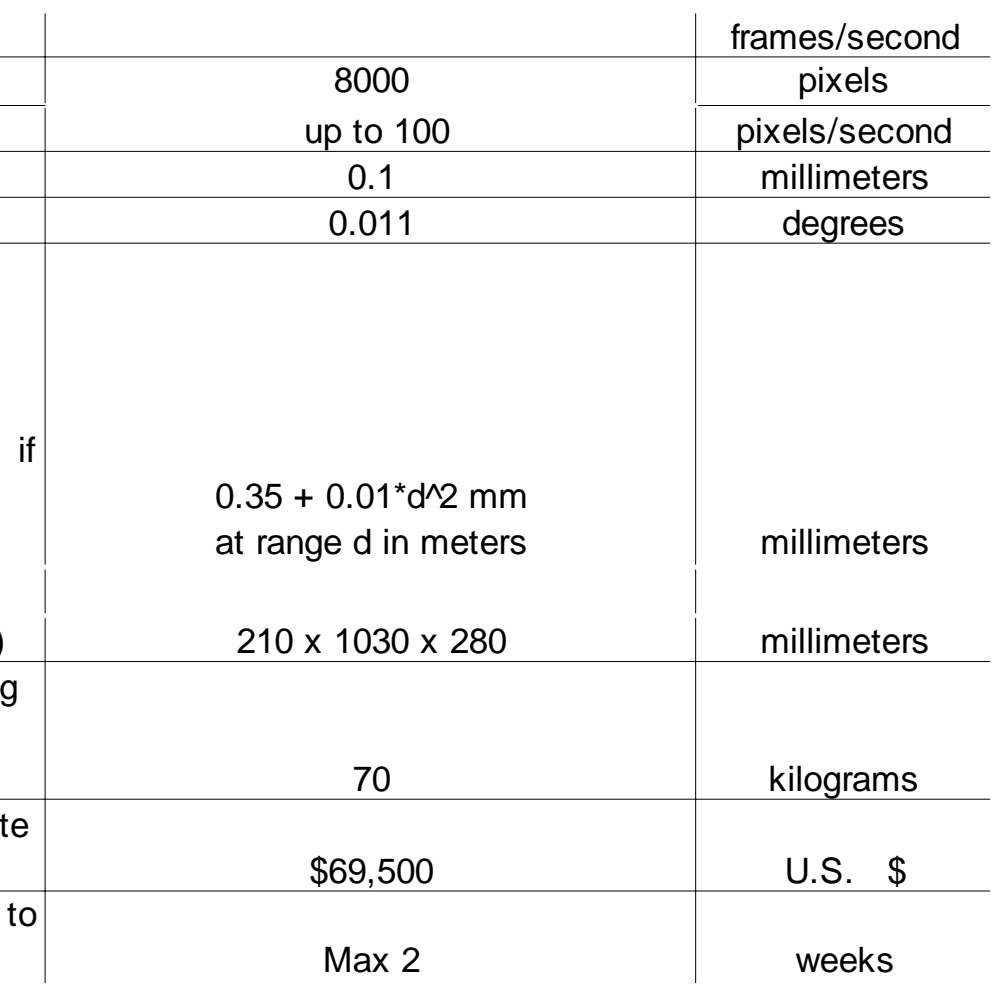
frames/second pixels pixels/second millimeters degrees

Overall size of unit (height, width, depth) Weight of the complete sensor, including power supplies and any required interfaces

Retail cost or Estimated cost to duplicate a lab prototype. Lead time to delivery (or estimated time to duplicate a lab prototype) 


\section{NIST 2004 LADAR TECHNOLOGY SURVEY}

\begin{tabular}{l|l|} 
Source Name & Arete Associates \\
\hline Source Address & $\begin{array}{l}333 \text { N Wilmot Rd. Tucson, } \\
\text { AZ } 85711\end{array}$ \\
\hline Source Contact Phone & (520) 571-8660 \\
\hline Source Email & garyredford@arete-az.com \\
\hline Source FAX & $(520) 571-8232$ \\
\hline $\begin{array}{l}\text { Name of Device/Product } \\
\text { ldentification (e.g. } \\
\text { model, make) }\end{array}$ & AQS-20XEOID Adaptation \\
\hline Year First Introduced & 2000
\end{tabular}

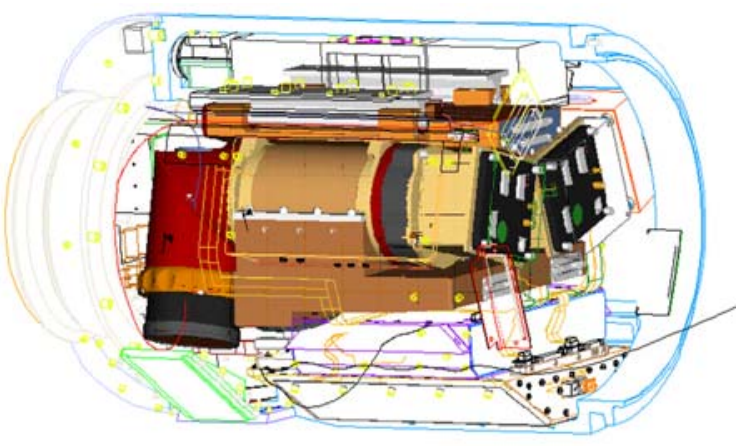

System Architecture

Streak Tube Imaging Lidar (STIL) that uses a streak tube with CCD camera to obtain push-broom scanned 3D imagery.

\begin{tabular}{l|c|c}
\hline & & \\
Wavelength of illumination source & 532 & nanometers \\
\hline $\begin{array}{l}\text { Illumination power } \\
\text { Source characteristic }\end{array}$ & 3 & Watts \\
$\begin{array}{l}\text { If pulse laser, specify pulse width and } \\
\text { repetition rate }\end{array}$ & Diode-pumped Nd:YAG laser & \\
Beam diameter at Exit & 20 & \\
\hline Beam divergence angle & 1.3 & millimeters \\
\hline $\begin{array}{l}\text { Sensor field of view, horizontal } \\
\text { Sensor field of view, vertical }\end{array}$ & Classified & degrees \\
\hline Maximum Range & $\sim 0.14$ & degrees \\
\hline
\end{tabular}

\begin{tabular}{|c|c|c|}
\hline Frame rate (if FPA) & 400 & frames/second \\
\hline Array size (if FPA) & $512 \times 128 \times 2$ & pixels (binned) \\
\hline Pixel rate (if scanner or other) & 400 lines/sec $\times 1024$ azimuth pixels/line & pixels/second \\
\hline Range resolution (depth) & 25.4 & millimeters \\
\hline Angular resolution & Classified & degrees \\
\hline $\begin{array}{l}\text { Range accuracy (depth) [note: if the } \\
\text { accuracy varies with range, please list } \\
\text { examples at several ranges; accuracy } \\
\text { should be reported at the } 1 \text {-sigma level } \\
\text { standard deviation about the true range; if } \\
\text { data are available at } 2 \text { and } 3 \text { sigma, } \\
\text { please provide those data] }\end{array}$ & $\sim 10$ & centimeters \\
\hline Overall size of unit (height, width, depth) & $380 \times 380 \times 509$ & millimeters \\
\hline $\begin{array}{l}\text { Weight of the complete sensor, including } \\
\text { power supplies and any required } \\
\text { interfaces }\end{array}$ & 64 & kilograms \\
\hline $\begin{array}{l}\text { Retail cost or Estimated cost to duplicate } \\
\text { a lab prototype. }\end{array}$ & $\$ 1,000,000$ & U.S. $\$$ \\
\hline $\begin{array}{l}\text { Lead time to delivery (or estimated time to } \\
\text { duplicate a lab prototype) }\end{array}$ & 52 & weeks \\
\hline
\end{tabular}




\section{NIST 2004 LADAR TECHNOLOGY SURVEY}

\begin{tabular}{|c|c|}
\hline Source Name & Arete Associates \\
\hline Source Address & $\begin{array}{l}333 \text { N Wilmot Rd. } \\
\text { Tucson, AZ } 85711\end{array}$ \\
\hline Source Contact Phone & (520) 571-8660 \\
\hline Source Email & agelbart@arete-az.com \\
\hline Source FAX & (520) $571-8232$ \\
\hline Name of Device/Product & $\begin{array}{l}\text { "PISTL" Polarimetric } \\
\text { Imaging Streak Tube } \\
\text { Ladar }\end{array}$ \\
\hline Identification (e.g. model & Prototype $\sqrt{2}$ \\
\hline Year First Introduced & 2000 \\
\hline
\end{tabular}

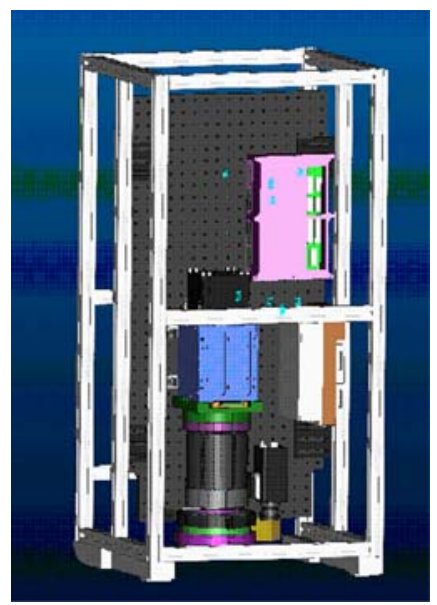

System Architecture

PISTL is a double-slit implementation of the Streak Tube Imaging Lidar (STIL) that uses a streak tube with CCD camera to obtain push-broom scanned 3D imagery. A Wollaston Prism in front of the imaging lens separates orthogonal polarimetric components simu

Wavelength of illumination source Illumination power

Source characteristic If pulse laser, specify pulse width and repetition rate

Beam diameter at Exit Beam divergence angle

Sensor field of view, horizontal Sensor field of view, vertical Maximum Range 532 nanometers

2000
milliWatts

Flash-lamp pumped Nd:YAG laser

\begin{tabular}{|c|c|c|}
\hline Frame rate (if FPA) & 100 & frames/second \\
\hline Array size (if FPA) & $512 \times 512$ & pixels \\
\hline Pixel rate (if scanner or other) & 100 lines/sec x 512 azimuth pixels/line & pixels/second \\
\hline Range resolution (depth) & 75 & millimeters \\
\hline Angular resolution & 0.05 & degrees \\
\hline $\begin{array}{l}\text { Range accuracy (depth) [note: if the } \\
\text { accuracy varies with range, please list } \\
\text { examples at several ranges; accuracy } \\
\text { should be reported at the } 1 \text {-sigma level } \\
\text { standard deviation about the true range; if } \\
\text { data are available at } 2 \text { and } 3 \text { sigma, } \\
\text { please provide those data] }\end{array}$ & $\begin{array}{l}\text { Does not currently have absolute } \\
\text { ranging capability (only relative ranging } \\
\text { with } 75 \mathrm{~mm} \text { precision over } 20 \mathrm{~m} \text { from } \\
100 \mathrm{~m} \text { altitude). }\end{array}$ & millimeters \\
\hline Overall size of unit (height, width, depth) & $1000 \times 1000 \times 875$ & millimeters \\
\hline $\begin{array}{l}\text { Weight of the complete sensor, including } \\
\text { power supplies and any required } \\
\text { interfaces }\end{array}$ & 200 & kilograms \\
\hline $\begin{array}{l}\text { Retail cost or Estimated cost to duplicate } \\
\text { a lab prototype. }\end{array}$ & $\$ 1,000,000$ & U.S. $\$$ \\
\hline $\begin{array}{l}\text { Lead time to delivery (or estimated time to } \\
\text { duplicate a lab prototype) }\end{array}$ & 24 & weeks \\
\hline
\end{tabular}




\section{NIST 2004 LADAR TECHNOLOGY SURVEY}

\begin{tabular}{l|l|} 
Source Name & $\begin{array}{l}\text { CYRA Technologies, } \\
\text { Inc. }\end{array}$ \\
\hline Source Address & $\begin{array}{l}\text { 4550 Norris Canyon } \\
\text { Road; San Ramon, CA } \\
94597 \text { USA }\end{array}$ \\
\hline Source Contact Phone & (866) 920-2300 \\
\hline Source Email & info@cyra.com \\
\hline Source FAX & (925) 790-2309 \\
\hline Name of Device/Product & CYRAX/ Leica \\
\hline Identification (e.g. model & Cyrax 2500 / HDS3000 \\
\hline Year First Introduced & $2000 / 2003$
\end{tabular}

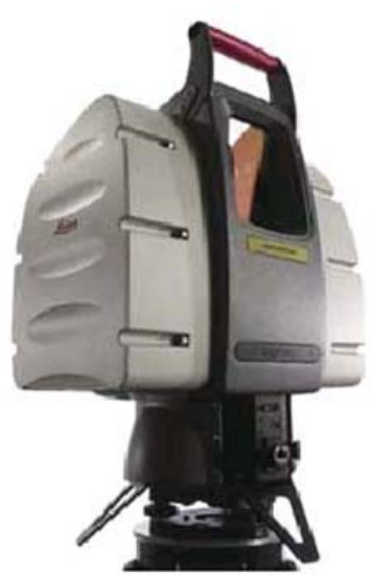

\begin{tabular}{l|c|c} 
System Architecture & Direct detection (pulse time of flight) & \\
\hline $\begin{array}{l}\text { Wavelength of illumination source } \\
\text { Illumination power }\end{array}$ & 532 & nanometers \\
$\begin{array}{l}\text { Source characteristic } \\
\text { If pulse laser, specify pulse width and } \\
\text { repetition rate }\end{array}$ & less than 1 & milliWatts \\
Beam diameter at Exit & & \\
\hline Beam divergence angle & 6 & millimeters \\
$\begin{array}{l}\text { Sensor field of view, horizontal } \\
\text { Sensor field of view, vertical }\end{array}$ & 0.1 & milli-radians \\
\hline Maximum Range & 40 & degrees \\
\hline
\end{tabular}

Frame rate (if FPA)

Array size (if FPA)

$\mathrm{N} / \mathrm{A}$ frames/second

Pixel rate (if scanner or other)

$\mathrm{N} / \mathrm{A}$
pixels

Range resolution (depth)

1000
pixels/second

Angular resolution Range accuracy (depth) [note: if the accuracy varies with range, please list examples at several ranges; accuracy should be reported at the 1-sigma level standard deviation about the true range; if data are available at 2 and 3 sigma, please provide those data]

Overall size of unit (height, width, depth) Weight of the complete sensor, including power supplies and any required interfaces $2 \mathrm{~mm}$ modeled surface millimeters

Retail cost or Estimated cost to duplicate a lab prototype. Lead time to delivery (or estimated time to duplicate a lab prototype)

millimeters

$381 \times 336.55 \times 429.26$ millimeters

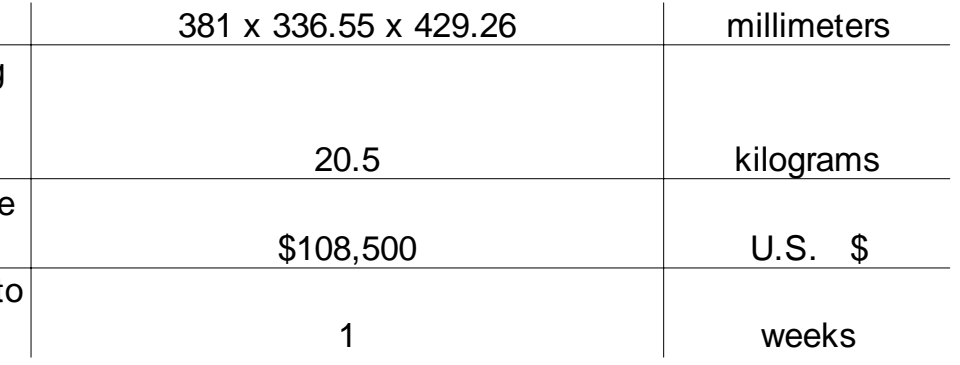




\section{NIST 2004 LADAR TECHNOLOGY SURVEY}

\begin{tabular}{l|l|} 
Source Name & $\begin{array}{l}\text { General Dynamics } \\
\text { Robotic Systems }\end{array}$ \\
\hline Source Address & $\begin{array}{l}\text { 1234 Tech Court } \\
\text { Westminster, MD } \\
21157\end{array}$ \\
\hline Source Contact Phone & $410-876-9200$ \\
\hline Source Email & info@gdrs.com \\
\hline Source FAX & $410-876-9470$ \\
\hline $\begin{array}{l}\text { Name of Device/Product } \\
\text { Identification (e.g. }\end{array}$ & Mobility Ladar \\
model, make) & Gen 2b \\
\hline
\end{tabular}

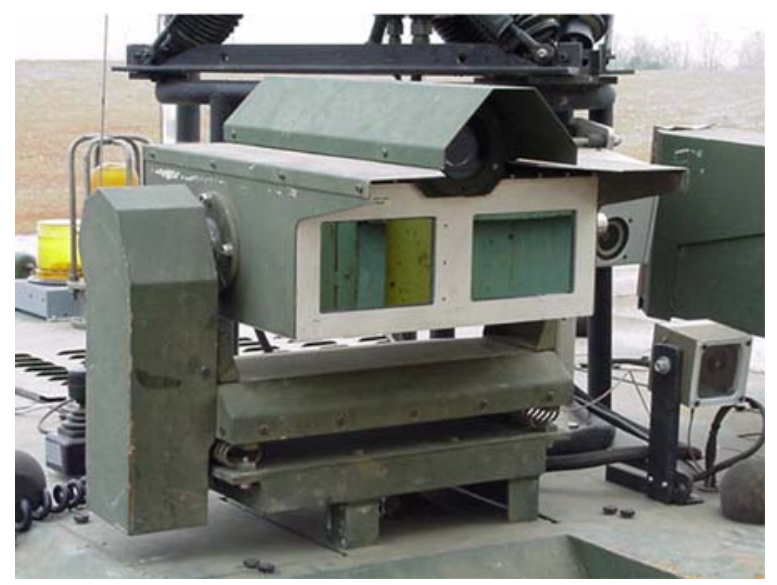

System Architecture

Digital multi-pulse, direct detection (pulse time of flight). Device uses

pulsed laser diode array active optics; rotating polygon scanner; and 16

\begin{tabular}{l|c|c}
\hline $\begin{array}{l}\text { Wavelength of illumination source } \\
\text { Illumination power }\end{array}$ & $\begin{array}{c}\text { Class } 1 \mathrm{~A} \\
\text { element linear detector array. }\end{array}$ & nanometers \\
$\begin{array}{l}\text { Source characteristic } \\
\text { If pulse laser, specify pulse width and } \\
\text { repetition rate }\end{array}$ & $\begin{array}{c}\text { milsed laser diode array (8 laser beams, } \\
1 \text { rotating polygon mirror) }\end{array}$ & \\
Beam diameter at Exit & 25 & millimeters \\
\hline Beam divergence angle & & milli-radians \\
Sensor field of view, horizontal & 180 pixels $=90$ degrees & degrees \\
\hline Sensor field of view, vertical & 32 pixels $=20$ degrees & degrees \\
\hline Maximum Range & $80 \mathrm{~m}(20 \%$ target reflectivity $)$ & meters
\end{tabular}

\begin{tabular}{|c|c|c|}
\hline Frame rate (if FPA) & $60 \mathrm{~Hz}$ & frames/second \\
\hline Array size (if FPA) & $32 \times 180$ (scanned) & pixels \\
\hline Pixel rate (if scanner or other) & 345,600 & pixels/second \\
\hline Range resolution (depth) & 76 mm (Gen2a); 20 mm (Gen 2b) & millimeters \\
\hline Angular resolution & $0.658 \times 0.5$ & degrees \\
\hline $\begin{array}{l}\text { Range accuracy (depth) [note: if the } \\
\text { accuracy varies with range, please list } \\
\text { examples at several ranges; accuracy } \\
\text { should be reported at the } 1 \text {-sigma level } \\
\text { standard deviation about the true range; if } \\
\text { data are available at } 2 \text { and } 3 \text { sigma, } \\
\text { please provide those data] }\end{array}$ & $\begin{array}{c}\text { +/- 76mm 1-sigma (Gen 2a) } \\
+/-20 \mathrm{~mm} 1 \text {-sigma (Gen } 2 \mathrm{~b} \text { and later) }\end{array}$ & millimeters \\
\hline Overall size of unit (height, width, depth) & approx. $152 \times 432 \times 356$ & millimeters \\
\hline $\begin{array}{l}\text { Weight of the complete sensor, including } \\
\text { power supplies and any required } \\
\text { interfaces }\end{array}$ & approx. 9 & kilograms \\
\hline $\begin{array}{l}\text { Retail cost or Estimated cost to duplicate } \\
\text { a lab prototype. }\end{array}$ & $\$ 92,569$ & U.S. \$ \\
\hline $\begin{array}{l}\text { Lead time to delivery (or estimated time to } \\
\text { duplicate a lab prototype) }\end{array}$ & Call for estimate & weeks \\
\hline
\end{tabular}




\section{NIST 2004 LADAR TECHNOLOGY SURVEY}

\begin{tabular}{l|l} 
Source Name & Sick, Inc. \\
\hline Source Address & $\begin{array}{l}6900 \text { West 110th } \\
\text { Street, Bloomington, } \\
\text { MN 55438 }\end{array}$ \\
\hline Source Contact Phone & $\begin{array}{l}1-800-325-7425, \\
+1(952) 941-6780\end{array}$ \\
\hline Source Email, Web Add & info@sw.sickusa.com, \\
\hline Source FAX & $+1(952)$ 941-9287 \\
\hline Name of Device/Product & Laser Measurement \\
\hline
\end{tabular}

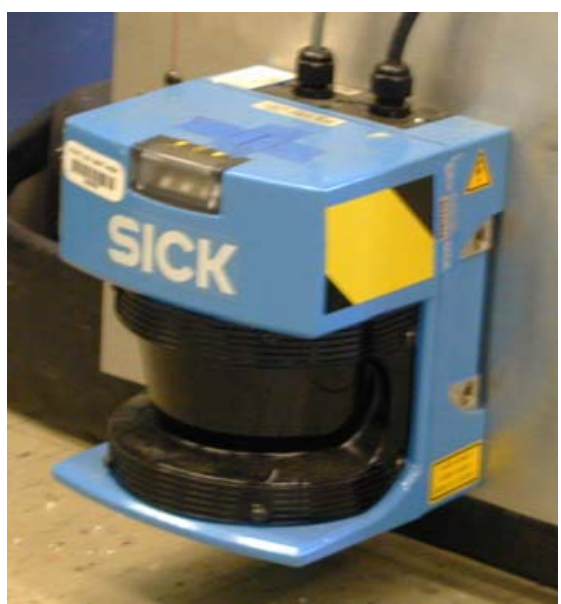

System Architecture

Line Scanner. Pulsed, time of flight light is sent and received per measurement angle $\left(1 / 4^{\circ}, 1 / 2^{\circ}, 1^{\circ}\right.$ increments) with respect to the unit. A rotating mirror moves the pulse to successive angles

\begin{tabular}{|c|c|c|}
\hline & throughout a $180^{\circ}$ pan. & \\
\hline Wavelength of illumination source & & nanometers \\
\hline Illumination power & & milliWatts \\
\hline Source characteristic & pulsed laser & \\
\hline $\begin{array}{l}\text { If pulse laser, specify pulse width and } \\
\text { repetition rate }\end{array}$ & & \\
\hline Beam diameter at Exit & & millimeters \\
\hline Beam divergence angle & & milli-radians \\
\hline Sensor field of view, horizontal & 180 & degrees \\
\hline Sensor field of view, vertical & n/a (1D scanner only) & degrees \\
\hline Maximum Range & $80 \mathrm{~m}(262.5 \mathrm{ft}) / 10 \mathrm{~m}(32.8 \mathrm{ft})$ & meters \\
\hline
\end{tabular}

\begin{tabular}{|c|c|c|}
\hline Frame rate (if $\mathrm{FPA}$ ) & $\begin{array}{c}\text { Programmable: } 3.3 \% / \mathrm{ms} @ 53 \mathrm{~ms} / . . . \\
\text { /13.8\% } / \mathrm{ms} @ 13 \mathrm{~ms}\end{array}$ & frames/second \\
\hline Array size (if FPA) & $180 / 360 / 720 \times 1$ (selectable) & pixels \\
\hline Pixel rate (if scanner or other) & $9.6 / 19.2$ / 38.4 / $500 \mathrm{kBd}$ (transfer rate) & bytes/second \\
\hline Range resolution (depth) & $10 \mathrm{~mm}(.39 \mathrm{in})$ & millimeters \\
\hline Angular resolution & $0.25^{\circ} / .5^{\circ} / 1^{\circ}$ (selectable) & degrees \\
\hline $\begin{array}{l}\text { Range accuracy (depth) [note: if the } \\
\text { accuracy varies with range, please list } \\
\text { examples at several ranges; accuracy } \\
\text { should be reported at the } 1 \text {-sigma level } \\
\text { standard deviation about the true range; if } \\
\text { data are available at } 2 \text { and } 3 \text { sigma, } \\
\text { please provide those data] }\end{array}$ & $\begin{array}{c}\text { Typical } \pm 15 \mathrm{~mm}(\mathrm{~mm} \text {-mode)} \text {, range } 1 \ldots 8 \\
\mathrm{m} ; \text { typical } \pm 40 \mathrm{~mm}(\mathrm{~cm}-\text { mode }) \text {, range } \\
1 \ldots 20 \mathrm{~m}\end{array}$ & millimeters \\
\hline Overall size of unit (height, width, depth) & $352 \times 228.5 \times 266 \mathrm{~mm}$ & millimeters \\
\hline $\begin{array}{l}\text { Weight of the complete sensor, including } \\
\text { power supplies and any required } \\
\text { interfaces }\end{array}$ & approx. $9 \mathrm{~kg}(19.8 \mathrm{lb})$ & kilograms \\
\hline $\begin{array}{l}\text { Retail cost or Estimated cost to duplicate } \\
\text { a lab prototype. }\end{array}$ & $\sim \$ 4000$ & U.S. \$ \\
\hline $\begin{array}{l}\text { Lead time to delivery (or estimated time to } \\
\text { duplicate a lab prototype) }\end{array}$ & 16 & weeks \\
\hline
\end{tabular}




\section{NIST 2004 LADAR TECHNOLOGY SURVEY}

\begin{tabular}{|c|c|}
\hline Source Name & OmniTech Robotics \\
\hline Source Address & $\begin{array}{l}2640 \text { South Raritan } \\
\text { Circle, Englewood, CO } \\
80110-1147\end{array}$ \\
\hline Source Contact Phone & (303) 922-7773 \\
\hline Source Email, Web Add & $\begin{array}{l}\text { David Parish } \\
\text { www.omnitech.com }\end{array}$ \\
\hline Source FAX & (303) 922-7775 \\
\hline Name of Device/Product & $\begin{array}{l}\text { SICK LMS with } \\
\text { OmniTech Tilt } \\
\text { mechanism }\end{array}$ \\
\hline Identification (e.g. model & Model 200 \\
\hline Year First Introduced & \\
\hline
\end{tabular}

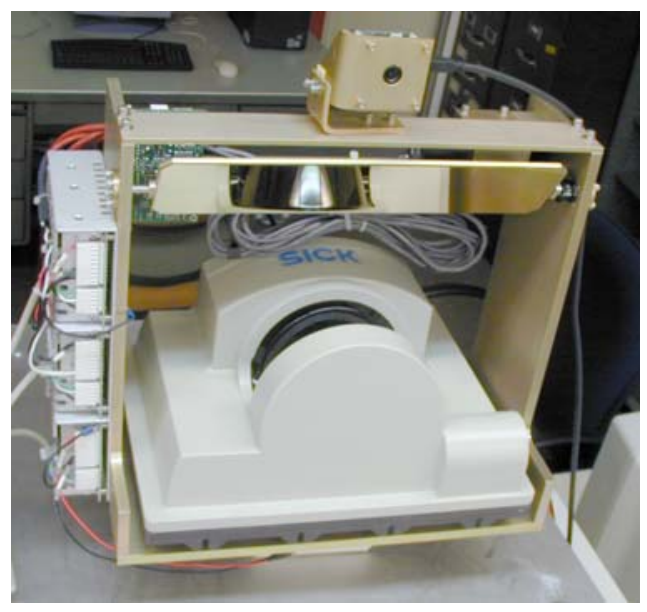

System Architecture

2D scanning version of the SICK ladar.

Pulsed, time of flight light is sent and received per measurement angle

$\left(1 / 4^{\circ}, 1 / 2^{\circ}, 1^{\circ}\right.$ increments) with respect to the unit. A rotating mirror moves the pulse

to successive angles throughout a $180^{\circ}$

\begin{tabular}{l|c|c} 
& \multicolumn{1}{|c}{ pan. } & \\
\hline $\begin{array}{l}\text { Wavelength of illumination source } \\
\text { Illumination power }\end{array}$ & & nanometers \\
$\begin{array}{l}\text { Source characteristic } \\
\text { If pulse laser, specify pulse width and } \\
\text { repetition rate }\end{array}$ & pulsed laser & \\
Beam diameter at Exit & & \\
\hline $\begin{array}{l}\text { Beam divergence angle } \\
\text { Sensor field of view, horizontal }\end{array}$ & & millimeters \\
\hline Sensor field of view, vertical & 80 & milli-radians \\
\hline Maximum Range & $80 \mathrm{~m}(262.5 \mathrm{ft}) / 10 \mathrm{~m}(32.8 \mathrm{ft})$ & degrees \\
\hline
\end{tabular}

\begin{tabular}{|c|c|c|}
\hline Frame rate (if FPA) & $\begin{array}{c}\text { Programmable: } 3.3 \% / \mathrm{ms} @ 53 \mathrm{~ms} / . . . \\
/ 13.8 \% \mathrm{~ms} @ 13 \mathrm{~ms}\end{array}$ & frames/second \\
\hline Array size (if FPA) & $180 / 360 / 720 \times 1$ (selectable) & pixels \\
\hline Pixel rate (if scanner or other) & 9.6 / 19.2 / 38.4 / 500 kBd (transfer rate) & bytes/second \\
\hline Range resolution (depth) & $10 \mathrm{~mm}(.39 \mathrm{in})$ & millimeters \\
\hline Angular resolution & $.25^{\circ} / .5^{\circ} / 1^{\circ}$ (selectable $)$ & degrees \\
\hline $\begin{array}{l}\text { Range accuracy (depth) [note: if the } \\
\text { accuracy varies with range, please list } \\
\text { examples at several ranges; accuracy } \\
\text { should be reported at the } 1 \text {-sigma level } \\
\text { standard deviation about the true range; if } \\
\text { data are available at } 2 \text { and } 3 \text { sigma, } \\
\text { please provide those data] }\end{array}$ & $\begin{array}{c}\text { Typical } \pm 15 \mathrm{~mm} \text { (mm-mode), range } 1 \ldots 8 \\
\mathrm{~m} ; \text { typical } \pm 40 \mathrm{~mm}(\mathrm{~cm} \text {-mode), range } \\
1 \ldots 20 \mathrm{~m}\end{array}$ & millimeters \\
\hline Overall size of unit (height, width, depth) & 457 w. $\times 394$ h. $\times 279$ d. & millimeters \\
\hline $\begin{array}{l}\text { Weight of the complete sensor, including } \\
\text { power supplies and any required } \\
\text { interfaces }\end{array}$ & 10 & kilograms \\
\hline $\begin{array}{l}\text { Retail cost or Estimated cost to duplicate } \\
\text { a lab prototype. }\end{array}$ & $\$ 50,000$ & U.S. $\$$ \\
\hline $\begin{array}{l}\text { Lead time to delivery (or estimated time to } \\
\text { duplicate a lab prototype) }\end{array}$ & Call for quote & weeks \\
\hline
\end{tabular}




\section{NIST 2004 LADAR TECHNOLOGY SURVEY}

\begin{tabular}{|c|c|}
\hline Source Name & iQvolution \\
\hline Source Address & $\begin{array}{l}22 \text { Blue Fox Lane, } \\
\text { Richboro, PA } 18954\end{array}$ \\
\hline Source Contact Phone & $215-396-7332$ \\
\hline Source Email & Tsatterley@iqvolution.com \\
\hline Source FAX & 215-396-1219 \\
\hline Name of Device/Product & iQsun \\
\hline $\begin{array}{l}\text { Identification (e.g. model, } \\
\text { make) }\end{array}$ & Model 880 \\
\hline Year First Introduced & 2003 \\
\hline
\end{tabular}

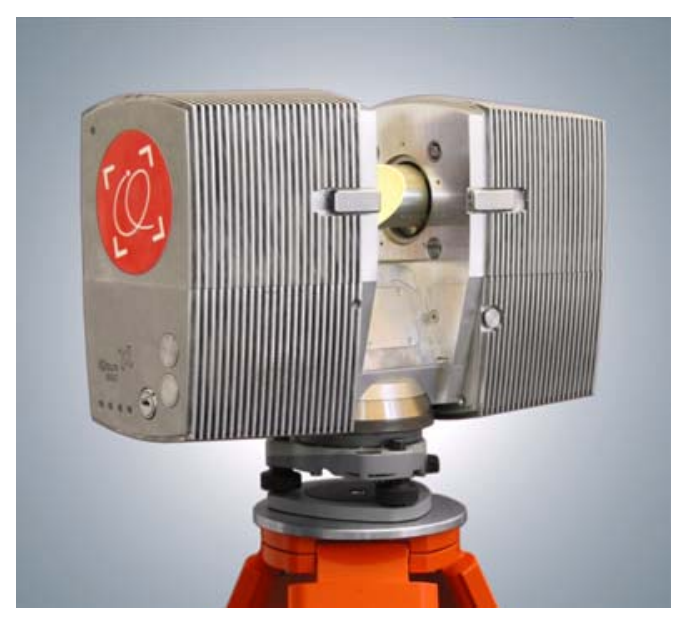

System Architecture

Continuous Wave scanner, modular in design, data recording on internal hard

disk. Class 3A Sacnning time $=4.5$ minutes per 360 revolution

Wavelength of illumination source Illumination power

Source characteristic

If pulse laser, specify pulse width and repetition rate

Beam diameter at Exit

Beam divergence angle

Sensor field of view, horizontal

Sensor field of view, vertical

Maximum Range

785

nanometers

\begin{tabular}{l|l}
10 & milliWatts
\end{tabular}

Contimuous Wave, Modular system

Frame rate (if FPA)

Array size (if FPA)

Pixel rate (if scanner or other)

Range resolution (depth)

Angular resolution

Range accuracy (depth) [note: if the

accuracy varies with range, please list

examples at several ranges; accuracy

should be reported at the 1-sigma level

standard deviation about the true range; if

data are available at 2 and 3 sigma,

please provide those data]

Overall size of unit (height, width, depth)

Weight of the complete sensor, including power supplies and any required

interfaces

Retail cost or Estimated cost to duplicate

a lab prototype.

Lead time to delivery (or estimated time to

duplicate a lab prototype)

to duplicate

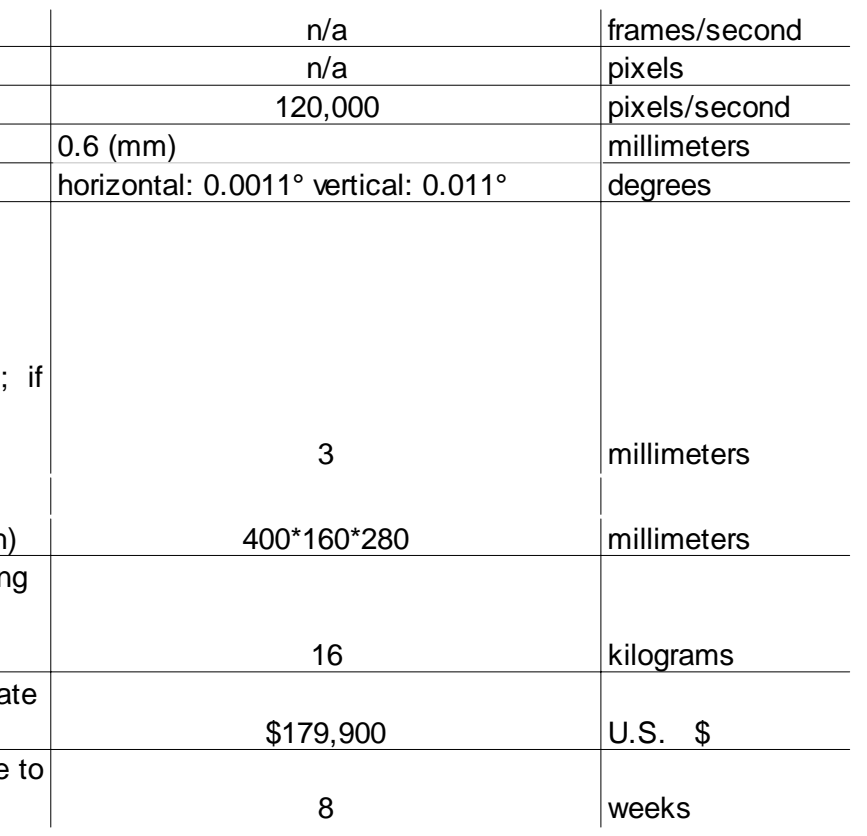




\section{Research Systems}




\section{NIST 2004 LADAR TECHNOLOGY SURVEY}

\begin{tabular}{l|l|} 
Source Name & $\begin{array}{l}\text { M.I.T. Lincoln } \\
\text { Laboratory }\end{array}$ \\
\hline Source Address & Lexington, MA, 02420 \\
\hline Source Contact Phone & 781-981-7945 \\
\hline Source Email & heinrichs@LL.mit.edu \\
\hline Source FAX & $781-981-5069$ \\
Name of Device/Product & $\begin{array}{l}\text { Geiger mode APD } \\
\text { (Photon Counting) }\end{array}$ \\
\hline model, make & $\begin{array}{l}\text { Gen 2, Jigsaw (Gen 3) } \\
\text { version }\end{array}$ \\
\hline Year First Introduced & 2001,2002
\end{tabular}

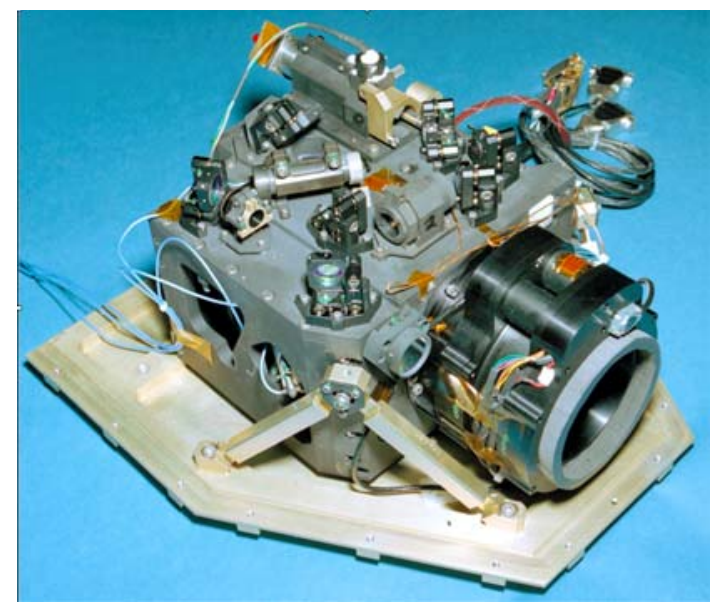

System Architecture

Wavelength of illumination source Illumination power

Source characteristic

If pulse laser, specify pulse width and repetition rate

Beam Diameter at Exit

Direct time-of -flight, $32 \times 32$ Silicon FPA with bump bonded CMOS ROIC array

Beam divergence angle (Far field - full)

Receiver aperture

Sensor field of view, horizontal

Sensor field of view, vertical

Total sensor field of view, scanned

Maximum Range

780,532

nanometers

15,5

uJ per pulse

Pulsed u-chip laser

$250 \mathrm{ps} \times 10 \mathrm{kHz}$

Gen 2: 20, Jigsaw: 20

Gen 2: 12, Jigsaw: 24

milli-radians

Gen 2: 100, Jigsaw: 75

Gen 2: 0.5, Jigsaw: $\sim 1.0$ Fixed optics

millimeters

Gen 2: 0.5, Jigsaw: $\sim 1.0$ Fixed optics

degrees

degrees

Gen 2: 20X16, Jigsaw: 12 circular

degrees

Gen 2: 1200, Jigsaw: 600

meters

Frame rate (if FPA)

Array size (if FPA)

$8 \mathrm{~K}, 10 \mathrm{~K}$

frames/second

Pixel rate (if scanner or other) $32 \times 32$ pixels

Range resolution (depth) up to $10,000,000$

Angular resolution

Range accuracy (depth)

Gen 2: 150, Jiagsaw: 300

Gen 2: 0.01, Jigsaw: 0.02

Gen 2: 150, Jiagsaw: 300 ixels/second millimeters degrees/pixel millimeters

Gen 2: 20,30,45, Jigsaw:21,38,37 plus

Overall size of unit (height, width, depth) heat exchanger $12,18,34$

centimeters

Weight of the complete sensor, including power supplies and any required interfaces

Gen 2: 18, Jigsaw: 28 kilograms

Retail cost or Estimated cost to duplicate a lab prototype. not available currently

Lead time to delivery (or estimated time to duplicate a lab prototype)

not available currently

U.S. $\$$

weeks 


\section{NIST 2004 LADAR TECHNOLOGY SURVEY}

\begin{tabular}{l|l|} 
& $\begin{array}{l}\text { Sandia National } \\
\text { Laboratories } \\
\text { SOC - Robert D. Habbit }\end{array}$ \\
\hline Source Address & $\begin{array}{l}\text { P.O. Box 5800 } \\
\text { Albuquerque, New } \\
\text { Mexico, USA 87185- } \\
\text { 0329 }\end{array}$ \\
\hline Source Contact Phone & (505)-845-9492 \\
\hline Source Email & rdhabbi@sandia.gov \\
\hline Source FAX & (505)-844-9554 \\
Name of Device/Product & LDRI \\
\hline
\end{tabular}

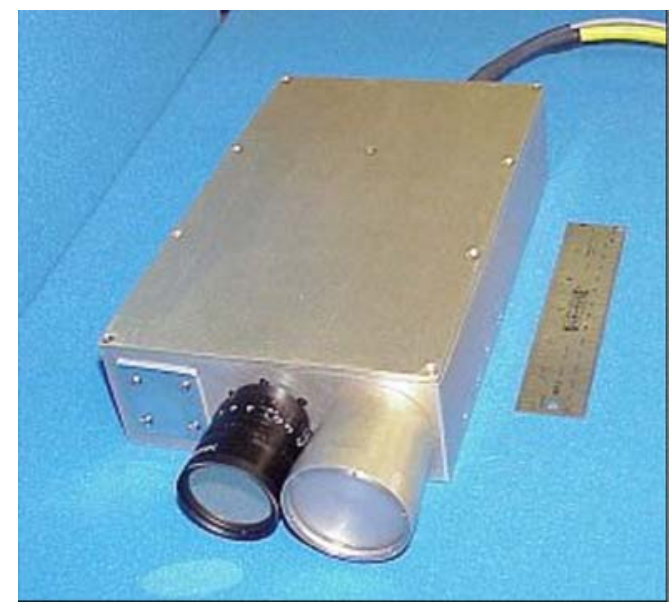

System Architecture

Scannerless Range Imaging utilizing a modulated intensified CCD Focal Plane Array and flood light illumination. The nature of the measurement process is multi-frequency AM-CW.

\begin{tabular}{|c|c|c|}
\hline Wavelength of illumination source & 805 & nanometers \\
\hline Illumination power & 12 & Watts \\
\hline Source characteristic & Modulated Laser Diode & \\
\hline \multicolumn{3}{|l|}{$\begin{array}{l}\text { If pulse laser, specify pulse width and } \\
\text { repetition rate }\end{array}$} \\
\hline Beam diameter at Exit & 3 & inches \\
\hline Beam divergence angle & 60 fwhm & degrees \\
\hline Sensor field of view, horizontal & 40 & degrees \\
\hline Sensor field of view, vertical & 28 & degrees \\
\hline Maximum Range & 150 & feet \\
\hline Frame rate (if $\mathrm{FPA}$ ) & 7.5 & frames/second \\
\hline Array size (if FPA) & $640 \times 480$ & pixels \\
\hline Pixel rate (if scanner or other) & & pixels/second \\
\hline Range resolution (depth) & 0.2 & inches \\
\hline Angular resolution & 0.0625 & degrees \\
\hline $\begin{array}{l}\text { Range accuracy (depth) [note: if the } \\
\text { accuracy varies with range, please list } \\
\text { examples at several ranges; accuracy } \\
\text { should be reported at the } 1 \text {-sigma level } \\
\text { standard deviation about the true range; if } \\
\text { data are available at } 2 \text { and } 3 \text { sigma, } \\
\text { please provide those data] }\end{array}$ & 0.2 & inches \\
\hline Overall size of unit (height, width, depth) & $3 \times 11 \times 6$ & Inches \\
\hline $\begin{array}{l}\text { Weight of the complete sensor, including } \\
\text { power supplies and any required } \\
\text { interfaces }\end{array}$ & 5 & Pounds \\
\hline $\begin{array}{l}\text { Retail cost or Estimated cost to duplicate } \\
\text { a lab prototype. }\end{array}$ & Please Call & U.S. \$ \\
\hline $\begin{array}{l}\text { Lead time to delivery (or estimated time to } \\
\text { duplicate a lab prototype) }\end{array}$ & Please Call & weeks \\
\hline
\end{tabular}




\section{NIST 2003 LADAR TECHNOLOGY SURVEY}

\begin{tabular}{l|l|} 
Source Name & $\begin{array}{l}\text { National Institute of } \\
\text { Standards and } \\
\text { Technology (NIST) }\end{array}$ \\
\hline Source Address & $\begin{array}{l}\text { Building 226 / B148, } \\
\text { MS-8611, Gaithersburg, } \\
\text { MD 20899 }\end{array}$ \\
\hline Source Contact Phone & $301-975-6075$ \\
\hline Source Email & william.stone@nist.gov \\
\hline Source FAX & $301-869-6275$ \\
\hline Name of Device/Product & FANDANGO \\
\hline model, make & Gen 1 \\
\hline Year First Introduced & 2003
\end{tabular}

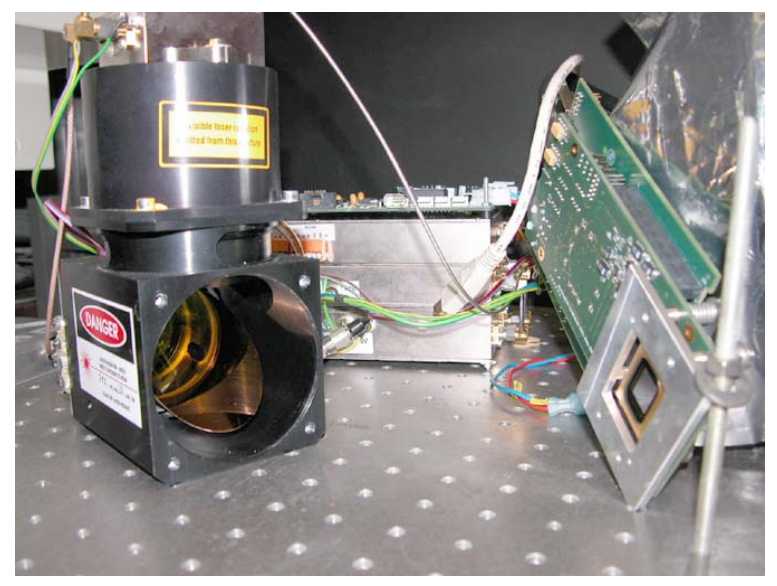

System Architecture

\begin{tabular}{l|c|c} 
& $\begin{array}{c}\text { beam deflector (presently configured as } \\
\text { an ultra-fast line-scanner) }\end{array}$ & nanometers \\
\hline Wavelength of illumination source & 780 & milliWatts \\
\hline Illumination power & 22 & millimeters \\
\hline Beam diameter at Exit & 3.5 & milli-radians \\
\hline Beam divergence angle & 0.1 & degrees \\
\hline Sensor field of view, horizontal & 0 to 24 & degrees \\
\hline Sensor field of view, vertical & n/a & meters
\end{tabular}

Frame rate (if FPA)

Array size (if FPA)

A) $\quad 42$

\begin{tabular}{l|r} 
Pixel rate (if scanner or other) & 625,000 \\
\hline Range resolution (depth) & 0.38 \\
\hline Anguar resolution
\end{tabular}

Angular resolution

Range accuracy (depth) [note: if the accuracy varies with range, please list examples at several ranges]

dual-frequency AM/CW with 1D MEMS dual-frequency AM/CW with 1D MEMS an ultra-fast line-scanner)

Overall size of unit (height, width, depth) Weight of the complete sensor, including power supplies and any required interfaces

Retail cost Lead time to delivery

1.6

\begin{tabular}{|c|c|}
\hline 000 & $\begin{array}{c}\text { linear } \\
\text { scans/second }\end{array}$ \\
\hline$a$ & pixels \\
\hline 000 & pixels/second \\
\hline 38 & millimeters \\
\hline 6 & degrees \\
\hline
\end{tabular}




\section{NIST 2004 LADAR TECHNOLOGY SURVEY}

\begin{tabular}{l|c}
$\begin{array}{l}\text { Source Name } \\
\text { Source Address }\end{array}$ & $\begin{array}{c}\text { Army Research Lab } \\
\text { 2800 Powder Mill Rd., } \\
\text { Adelphi, MD }\end{array}$ \\
\hline Source Contact Phone & 301-394-3141 \\
\hline Source Email & stann@arl.army.mil \\
\hline Source FAX & $310-394-5270$ \\
Name of Device/Product & ARL chirped AM ladar \\
\hline Identification (e.g. model, m & None \\
\hline Year First Introduced & 2002
\end{tabular}

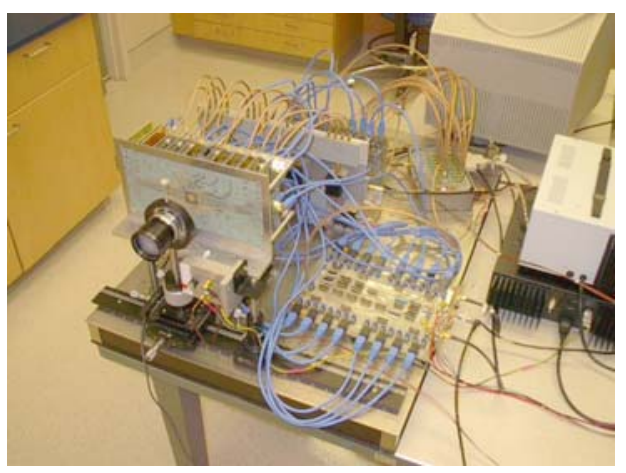

\begin{tabular}{|c|c|c|}
\hline System Architecture & $\begin{array}{l}\text { Chirped AM ranging, } 32 \times 32 \text { GaAs FPA, } \\
\text { Will retro fit InGaAs array when available }\end{array}$ & \\
\hline Wavelength of illumination source & 807,1550 & nanometers \\
\hline Illumination power & 4., 1. & milliWatts \\
\hline Beam diameter at Exit & 7 & millimeters \\
\hline Beam divergence angle & Variable & milli-radians \\
\hline Sensor field of view, horizontal & Variable with optics changes & degrees \\
\hline Sensor field of view, vertical & Variable with optics changes & degrees \\
\hline Maximum Range & $\begin{array}{l}1-2 \mathrm{~km} \text { with } 10 \mathrm{~W} \text { laser } \\
100 \mathrm{~m} \text { with } 1 \mathrm{~W} \text { laser }\end{array}$ & meters \\
\hline Frame rate (if FPA) & 3.3-10 & frames/second \\
\hline Array size (if FPA) & $32 \times 32$ & pixels \\
\hline Pixel rate (if scanner or other) & $3300-10000$ & pixels/second \\
\hline Range resolution (depth) & 250 & millimeters \\
\hline Angular resolution & Variable & degrees \\
\hline $\begin{array}{l}\text { Range accuracy (depth) [note: if the } \\
\text { accuracy varies with range, please list } \\
\text { examples at several ranges; accuracy } \\
\text { should be reported at the } 1 \text {-sigma level } \\
\text { standard deviation about the true range; if } \\
\text { data are available at } 2 \text { and } 3 \text { sigma, } \\
\text { please provide those data] }\end{array}$ & $10 @$ less than 100 m & millimeters \\
\hline Overall size of unit (height, width, depth) & Laboratory breadboard & millimeters \\
\hline $\begin{array}{l}\text { Weight of the complete sensor, including } \\
\text { power supplies and any required } \\
\text { interfaces }\end{array}$ & Laboratory breadboard & kilograms \\
\hline $\begin{array}{l}\text { Retail cost or Estimated cost to duplicate } \\
\text { a lab prototype. }\end{array}$ & $\$ 100,000$ & U.S. $\$$ \\
\hline $\begin{array}{l}\text { Lead time to delivery (or estimated time to } \\
\text { duplicate a lab prototype) }\end{array}$ & 52 & weeks \\
\hline
\end{tabular}




\section{NIST 2004 LADAR TECHNOLOGY SURVEY}

\begin{tabular}{|c|c|}
\hline Source Name & $\begin{array}{l}\text { Night Vision and } \\
\text { Electronic Sensors Dir. }\end{array}$ \\
\hline Source Address & $\begin{array}{l}\text { AMSEL-RD-NV-ST-LB } \\
\text { (Barr), Ft. Belvoir, VA }\end{array}$ \\
\hline Source Contact Phone & NA \\
\hline Source Email & NA \\
\hline Source FAX & NA \\
\hline Name of Device/Product & $\begin{array}{l}\text { High Resolution, Multi- } \\
\text { Pulse Laser Radar }\end{array}$ \\
\hline \multicolumn{2}{|l|}{$\begin{array}{l}\text { Identification (e.g. model, } \\
\text { make) }\end{array}$} \\
\hline Year First Introduced & 2001 \\
\hline
\end{tabular}

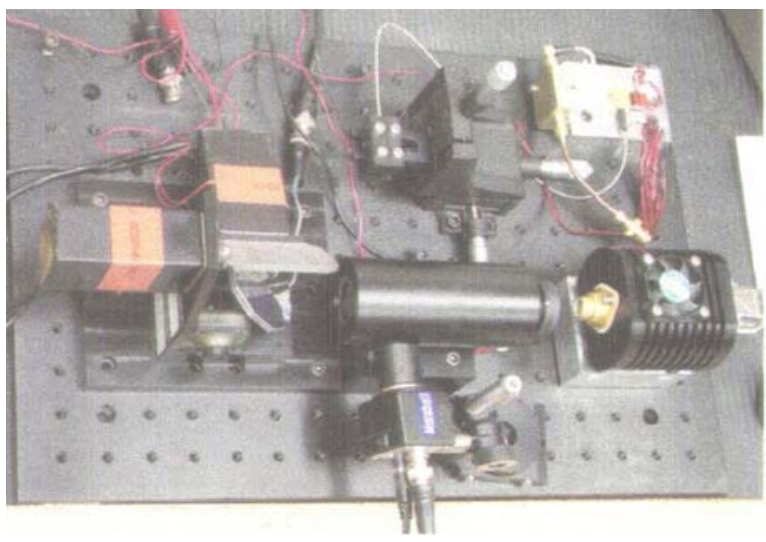

System Architecture

Scanning single beam; Nd:YAG microchip laser; InGaAs APD detector; programmable $x$,y galvo scanners; $2 \mathrm{GHz}$ digitization (8-bits); digitally records up to the full duration of the return signal;

$500 \mathrm{MHz}$ bandwidth; pan and tilt pointing; remote control via wi

\begin{tabular}{c|c|c}
\hline Wavelength of illumination source & 1064 & nanometers \\
\hline Illumination power & 6 & kW (peak) \\
Beam diameter at Exit & 22 & millimeters \\
\hline Beam divergence angle & 0.1 & milli-radians \\
& & \\
Sensor field of view, horizontal & Up to $+/-20 \quad$ (programmable) & degrees \\
\hline Sensor field of view, vertical & Up to $+/-20 \quad$ (programmable) & degrees \\
\hline Maximum Range & 150 & meters
\end{tabular}

\begin{tabular}{|c|c|c|}
\hline Frame rate (if FPA) & Depends on number of pixels & frames/second \\
\hline Array size (if FPA) & Single scanned beam & pixels \\
\hline Pixel rate (if scanner or other) & Nominally 1000 (w/o averaging) & pixels/second \\
\hline Range resolution (depth) & 250 & millimeters \\
\hline Angular resolution & 0.00057 & degrees \\
\hline $\begin{array}{l}\text { Range accuracy (depth) [note: if the } \\
\text { accuracy varies with range, please list } \\
\text { examples at several ranges; accuracy } \\
\text { should be reported as } 1 \text {-sigma level; if } \\
\text { more detailed information is available at } 2 \\
\text { and } 3 \text { sigma, please provide] }\end{array}$ & Nominally 75 (based on sample rate) & millimeters \\
\hline Overall size of unit (height, width, depth) & $600 \times 600 \times 200$ (head only) & millimeters \\
\hline $\begin{array}{l}\text { Weight of the complete sensor, including } \\
\text { power supplies and any required } \\
\text { interfaces }\end{array}$ & 9 (head only) & kilograms \\
\hline $\begin{array}{l}\text { Retail cost or Estimated cost to duplicate } \\
\text { a lab prototype. }\end{array}$ & $\begin{array}{c}\$ 27,000 \text { (COTS parts only, without pan } \\
\text { and tilt) }\end{array}$ & U.S. $\$$ \\
\hline $\begin{array}{c}\text { Lead time to delivery (or estimated time to } \\
\text { duplicate a lab prototype) }\end{array}$ & Not for sale & weeks \\
\hline
\end{tabular}




\section{NIST 2004 LADAR TECHNOLOGY SURVEY}

\begin{tabular}{|c|c|}
\hline Source Name & $\begin{array}{l}\text { Sandia National } \\
\text { Laboratories } \\
\text { POC - Robert D. Habbit }\end{array}$ \\
\hline Source Address & $\begin{array}{l}\text { P.O. Box } 5800 \\
\text { Albuquerque, New } \\
\text { Mexico, USA } 87185- \\
0329\end{array}$ \\
\hline Source Contact Phone & $(505)-845-9492$ \\
\hline Source Email & rdhabbi@sandia.gov \\
\hline Source FAX & (505)-844-9554 \\
\hline Name of Device/Product & SRI Flash Quad \\
\hline \multicolumn{2}{|l|}{$\begin{array}{l}\text { Identification (e.g. } \\
\text { model, make) }\end{array}$} \\
\hline Year First Introduced & 2001 \\
\hline
\end{tabular}

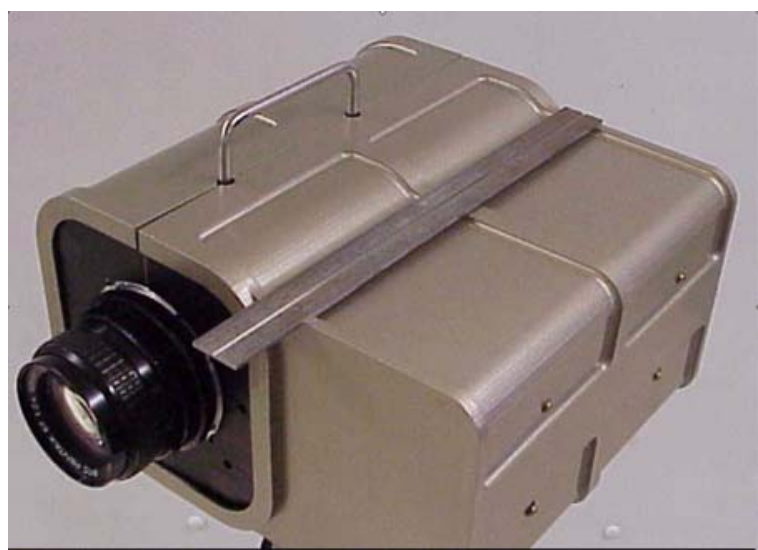

System Architecture

Scannerless Range Imaging utilizing 4 parallel modulated intensified CCD Focal Plane Arrays with flood light illumination. The nature of the measurement process is multi-frequency AM-CW.

Wavelength of illumination source Illumination power

Source characteristic If pulse laser, specify pulse width and repetition rate

Beam diameter at Exit Beam divergence angle

Sensor field of view, horizontal Sensor field of view, vertical Maximum Range is mult-requency AM-CW.

Frame rate (if FPA)

Array size (if FPA)

Pixel rate (if scanner or other)

Range resolution (depth)

Angular resolution

Range accuracy (depth) [note: if the accuracy varies with range, please list examples at several ranges; accuracy should be reported at the 1-sigma level standard deviation about the true range; if data are available at 2 and 3 sigma, please provide those data]

please list
; accuracy
sigma level
true range;
sigma,

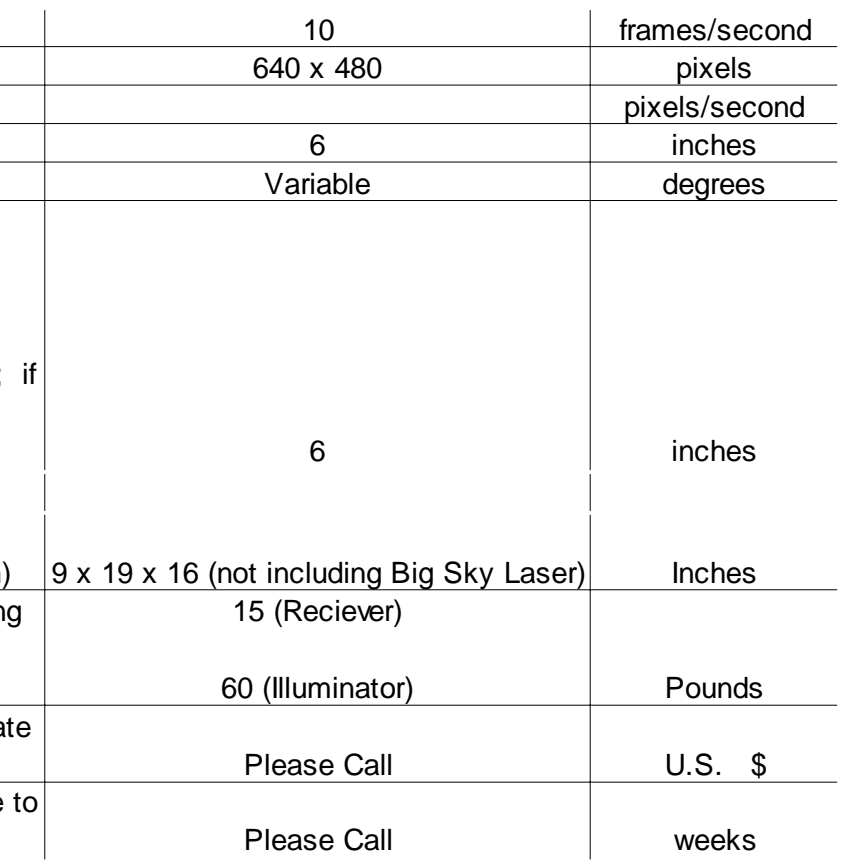

Overall size of unit (height, width, depth) $9 \times 19 \times 16$ (not including Big Sky Laser) Inches Weight of the complete sensor, including power supplies and any required interfaces

Retail cost or Estimated cost to duplicate a lab prototype. Lead time to delivery (or estimated time to duplicate a lab prototype)

Please Call 


\title{
Appendix B
}

\author{
Next Generation LADAR for \\ Driving Unmanned Ground Vehicles
}

\section{BROAD AGENCY ANNOUNCEMENT}

National Institute of Standards and Technology

original 2002 solicitation

For Reference Only 


\section{B.1 Introduction}

The National Institute of Standards and Technology is soliciting proposals on Next Generation LADAR for Driving Unmanned Ground Vehicles.Based on the experience gained in support of the Army Research Lab (ARL) Demo III Unmanned Ground Vehicle program over the past four years, the Intelligent Systems Division at the National Institute of Standards and Technology (NIST) has identified the need for high performance LADAR sensors for autonomous on and off-road driving. These sensors will be mounted on UGVs and are needed to provide reliable and robust obstacle/object detection and terrain feature information. The sensors will provide information about the environment surrounding the vehicle sufficient to enable the vehicle to drive autonomously. Current LADAR sensors used in UGV research have proven to have limitations in performance, are large in size and are expensive. Phase I of this solicitation is for the design of an Engineering Prototype to advance the LADAR range imaging component/subsystem technologies to meet the needs for UGVs. Phase II will support prototype development.

The Government envisions the requirement for two types of LADAR range imaging sensors for this type of application, one having a wide field-of-view (40 X 90 deg.) with a resolution of about .25 degrees or better per pixel and the second, a foveal LADAR having a narrow field-of-view of approximately $1 / 10$ th of the wide field-of-view with a resolution of about .05 degrees or better per pixel. Inter-pixel spacing of no more than $10 \%$ of the specified spatial resolution is anticipated. The intent is to make the foveal LADAR steerable to any position within the field-of-view of the wide angle LADAR. Both types of LADAR sensors shall have a range resolution of about $5 \mathrm{~cm}$ in range, shall be able to detect the ground plane out to a distance of at least 40 meters and vertical surfaces out to a range of at least 100 meters. $10 \mathrm{~Hz}$ frame rates or better are expected. Both LADARs must be eye safe and shall be provided with capability to penetrate dust, fog, grass and light foliage (either by sensing multiple returns or looking for the last return) and be able to operate in full sunlight conditions. The criterion for scoring the proposed designs are provided below.

Two Phases are planned for this solicitation. Phase I is intended to obtain designs. Phase II may proceed to fund a prototype(s). In Phase I, in response to this solicitation, responders are to submit proposals describing their intended design concept and their capabilities to perform the design. Full designs are not sought at this point, however, the concept must be capable of leading to a design (and possibly a prototype) that can address the technical specifications provided below. The concept description must include relevant analytical explanation and/or experimental data required to support the concept. The concept description must make clear the principals of operation, attest to the scientific validity of the approach, and show how the proposed concept shall support the specifications. These proposals shall be evaluated and one or more responders may be funded to develop a full design for their concept(s). In Phase II, 
the completed designs shall be evaluated, and an award or awards may be made to developers of selected designs to build a prototype(s) according to the selected design(s).

The following describes the Phase I and Phase II components of this solicitation in more detail:

\section{Phase I: Detailed concept design for Wide FOV LADAR, Narrow (foveal) LADAR or for the combination of both}

\section{Concept Proposal:}

1) Proposals for the development of designs shall be reviewed and scored.

2) As many as four awards to develop and provide designs may be given depending on proposal quality and available funding.

\section{Design:}

3) Award funding level will be at least $\$ 50 \mathrm{~K}$ for each award to provide a design. Higher award amounts are possible and will be based on "best value" to the Government.

4) Within three months of an award, the offeror shall provide a detailed design for NIST to review.

5) A budget and schedule plan (including when a prototype will be available for testing) shall be provided with the design.

6) As part of the design, the offeror shall identify proposed computer interfaces necessary to interact with the sensor or sensor platform components, and describe the proposed software organization and proposed algorithms. Capability to be synchronized with external timing source shall also be supported by the design.

\section{Evaluation of Phase I}

The criteria and weights to be used for evaluation of proposals to develop a design will be:

1) Soundness, technical merit, and innovation (Maximum 40 points)

In addition to the general technical soundness of the proposed concept, technical merit includes the likelihood that the proposed concept can lead to a design (and possibly eventual prototype) that can score well with respect to the specifications given below.

2) Qualifications of the proposed technical staff to perform proposed conceptual design (Maximum 30 points) Includes evaluation of experience with LADAR systems and similar or related system design experience, knowledge of LADAR measurement and performance issues, and knowledge of current approaches. 
3) Potential for use in military UGV applications and product development (Maximum 30 points) Degree to which concept can be applicable to unmanned ground vehicle systems. Proposer shall show knowledge of technical issues associated with this application domain, and indicate how they will be addressed.

The following specifications define the performance capabilities desired in the next generation LADAR systems being sought. In Phase I, the submitted design concepts will be evaluated according to the criteria above, which includes the likelihood that the proposed concept can lead to a design (and possibly eventual prototype) that can score well with respect to these specifications. In Phase II, these specifications and the associated points shown, will be used to score full designs as the means to evaluate the full designs for possible awards to build a prototype(s).

\section{Ladar Specifications (and Phase II Points):}

The first two items are for the Wide FOV LADAR. The next two are for the Narrow FOV LADAR. The Wide FOV and Narrow FOV designs will be scored separately, whether they are for part of a single, combined system or are for independent Wide FOV and Narrow FOV systems.

\begin{tabular}{c|c|c|c|c}
$\begin{array}{c}\text { Wide Field-of-view } \\
\text { Ladar }\end{array}$ & $\begin{array}{c}\text { Vertical FOV } \\
\text { (degrees) }\end{array}$ & Points & $\begin{array}{c}\text { Horizontal FOV } \\
\text { (degrees) }\end{array}$ & Points \\
\hline & 40 & 4 & 90 & 4 \\
\hline & $30<$ FOV $<40$ & 3 & $60<$ FOV $<90$ & 3 \\
\hline & $<40$ & 0 & $<60$ & 5 \\
\hline & $>40$ & 5 & & \\
\hline & & & & \\
\hline & $\begin{array}{c}\text { Spatial Pixel Resolution } \\
\text { (degrees) }\end{array}$ & Points & & \\
\hline & $0.25-0.3$ & 12 & &
\end{tabular}

Narrow (foveal) FOV LADAR: If a design for a foveal LADAR is offered, the proposed FOV will be rated on technical merit in combination with the proposed spatial resolution. A maximum of 10 points is possible. It is expected that the FOV shall not be less than $4 \times 4$ deg. or larger than $9 \times 9$ deg.

\begin{tabular}{c|c|c}
$\begin{array}{c}\text { Narrow Field-of-view } \\
\text { LADAR }\end{array}$ & $\begin{array}{c}\text { Spatial Pixel Resolution } \\
\text { (degrees) }\end{array}$ & Points \\
\hline & $0.05-0.06$ & 12 \\
\hline & $<0.06$ & 15 \\
\hline & $0.06-0.1$ & 8 \\
\hline & $>0.1$ & 0
\end{tabular}


The following specifications are common to either the Wide FOV or Narrow FOV LADAR

\begin{tabular}{|c|c|c|}
\hline Range resolution & $5-10 \mathrm{~cm}$ & 3 \\
\hline (over min. to max. & $>10 \mathrm{~cm}$ & 0 \\
\hline measurement range) & $<5 \mathrm{~cm}$ & 5 \\
\hline $\begin{array}{l}\text { Ground detection maximum } \\
\text { range }\end{array}$ & $>50 m$ & 10 \\
\hline (sensor $1.5 \mathrm{~m}$ above flat & $40-50 m$ & 5 \\
\hline ground surface - gravel, dirt, & $<40 m$ & 0 \\
\hline \multicolumn{3}{|l|}{ or mowed grass) } \\
\hline Vertical surface detection & $125-200 m$ & 12 \\
\hline \multirow[t]{3}{*}{ maximum range } & $>200 m$ & 15 \\
\hline & $100-124 m$ & 8 \\
\hline & $<100 m$ & 0 \\
\hline \multirow[t]{3}{*}{ Minimum range } & $<1 \mathrm{~m}$ & 5 \\
\hline & $1-2 m$ & 3 \\
\hline & $>2 m$ & 0 \\
\hline \multirow[t]{4}{*}{ Frame rate } & $<5 \mathrm{~Hz}$ & 0 \\
\hline & $5-9 \mathrm{~Hz}$ & 8 \\
\hline & $10-20 \mathrm{~Hz}$ & 12 \\
\hline & $>20 \mathrm{~Hz}$ & 15 \\
\hline Laser wavelength & In $1500 \mathrm{~nm}$ range & 10 \\
\hline (note laser power / lasers & $\begin{array}{c}\text { other wavelength not } \\
\text { detectable by night vision } \\
\text { equipment }\end{array}$ & 8 \\
\hline \multirow[t]{2}{*}{ must be eye safe) } & $\begin{array}{c}\text { Other wavelengths not in } \\
\text { visible }\end{array}$ & 5 \\
\hline & $\begin{array}{c}\text { other wavelengths in } \\
\text { visible }\end{array}$ & 0 \\
\hline Interference prevention & Has prevention & 5 \\
\hline between multiple active & No prevention interference & 0 \\
\hline sensors & Potential solution provided & 3 \\
\hline \multirow[t]{3}{*}{ Reflectance image } & $\begin{array}{l}\text { Yes, scoring will depend } \\
\text { on quality }\end{array}$ & 15 \\
\hline & No & 0 \\
\hline & Potential solution provided & 8 \\
\hline \multirow[t]{3}{*}{$\begin{array}{l}\text { Dust, smoke, foliage } \\
\text { penetration }\end{array}$} & $\begin{array}{l}\text { yes, scoring will depend } \\
\text { on technical quality of } \\
\text { approach }\end{array}$ & 15 \\
\hline & no & 0 \\
\hline & potential solution provided & 8 \\
\hline
\end{tabular}


Maximum possible score of each type of LADAR for technical specifications:

\section{Other Design Considerations:}

Acceptable designs will be determined through evaluation of the above criteria. Among the acceptable designs, additional points are available to those designs that may optionally address the following:

Since the LADARs sought are engineering prototypes, requirements on size, weight, power, shock and vibration, protection from rain/humidity and temperature range of operation are not critical. However, since the ultimate intent is for vehicle-based systems, up to 10 additional points will be awarded for proposed designs according to their feasibility of implementation on Demo III XUV type (2500 - $3000 \mathrm{lb}$.) vehicles, where size, weight, power, shock and vibration, protection from rain/humidity and temperature range of operation will be important. It is anticipated that the size of the production prototypes for that purpose will need to be smaller than a cylinder $(20-25 \mathrm{~cm}$. high and about $12 \mathrm{~cm}$. across)

If a design for a foveal pan/tilt stage is offered (in addition to the LADAR imager), up to 10 additional points will be awarded according to its technical merit. Servos in the stage shall be capable of at least 3 saccades (point to point moves) per second. Designs for image stabilization must also be provided.

\section{Phase II: Engineering Prototype Development}

The designs developed under Phase I award(s) will be evaluated according to the specifications and points above. Based on the quality of the designs and availability of funding, NIST will consider funding one or more contracts to build a prototype Wide FOV LADAR, a Narrow (foveal) LADAR or a combination of both. We anticipate making Phase II award selections before the end of the calendar year. For any prototype that is developed, the offeror shall fully describe interfaces necessary to interact with the sensor platform components, and provide software required to control components. Capability to be synchronized with external timing source shall also be provided.

\section{Proposal Due Date}

This notice constitutes a BAA as contemplated in Federal Acquisition Regulation 6.102(d)(2)(i). A formal Request for Proposal or other solicitation regarding this announcement will not be issued, nor will paper copies of this announcement be issued. The Government reserves the right to select or award all, some or none of the proposals received in response to this announcement. All responsible sources may submit a proposal, which shall be considered by the agency. Proposals shall be received by 3:00 PM on July 9, 2002. 